\title{
Transport properties in nanostructured thermoelectric materials and single crystal perovskites
}

Author:

Michael Ng
Supervisor:

Dr. Jonathan Halpert

A thesis submitted to Victoria University of Wellington in partial fulfilment of the requirements for the degree of Master of Science in Physics in the

$$
\text { Halpert Group }
$$

School of Chemical and Physical Sciences

Victoria University of Wellington

$$
2017
$$

TE WHARE WĀNANGA O TE ÜPOKO O TE IKA A MĀUI

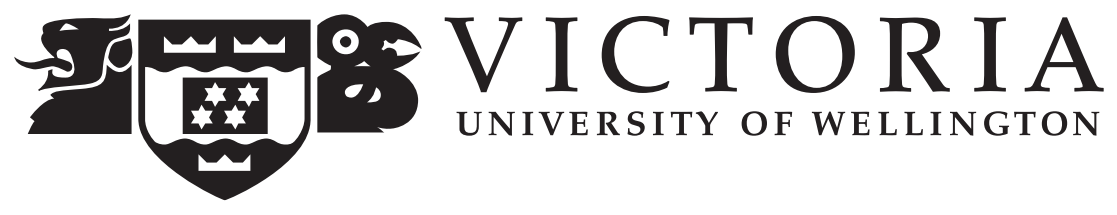





\section{Abstract}

Energy consumption worldwide is constantly increasing, bringing with it the demand for low cost, environmentally friendly and efficient energy technologies. One of these promising technologies is thermoelectrics in which electric power is harvested from waste heat energy. The efficiency of a thermoelectric device is determined by the dimensionless figure of merit $Z T=\sigma S^{2} T / \kappa$ where $\sigma$ is the electrical conductivity, $S$ is the thermopower, $\kappa$ is the thermal conductivity, and $T$ is the average temperature. In this thesis we investigate the use of nanostructuring, which has been known to lead to significant reduction in the lattice thermal conductivity to maximise the figure of merit.

One of the most successful bulk thermoelectric materials is $\mathrm{Bi}_{2} \mathrm{Te}_{3}$, with a $Z T$ of unity at room temperature. Here we investigate the effects of nanostructuring on the thermoelectric properties of $\mathrm{Bi}_{2} \mathrm{Te}_{3}$. Sub-100 nm Bi $\mathrm{Te}_{3}$ nanoparticles were successfully synthesized and the figure of merit was found to be $Z T \sim 5 \times 10^{-5}$ at room temperature. The effect of a ligand exchange treatment to replace the long chain organic ligand on the as-synthesized nanoparticles with a short chain alkyl ligand was explored. After ligand exchange treatment with hydrazine the figure of merit of sub- $100 \mathrm{~nm} \mathrm{Bi}_{2} \mathrm{Te}_{3}$ was found to increase by two fold to $Z T \sim 1 \times 10^{-4}$ at room temperature. Overall the figure of merit is low compared to other nanostructured $\mathrm{Bi}_{2} \mathrm{Te}_{3}$, this was attributed to the extremely low electrical conductivity. The thermopower and thermal conductivity were found to be $\sim 96 \mu \mathrm{V} \mathrm{K}^{-1}$ and $\sim 0.38 \mathrm{~W} \mathrm{~m}^{-1} \mathrm{~K}^{-1}$ at $300 \mathrm{~K}$ respectively, which show improvements over other nanostructured $\mathrm{Bi}_{2} \mathrm{Te}_{3}$.

Further optimisation of the figure of merit was also investigated by incorporating $\mathrm{Cu}, \mathrm{Ni}$ and $\mathrm{Co}$ dopants. The most successful of these attempts was Co in which $14.5 \%$ Co relative to Bi was successfully incorporated into sub-100 $\mathrm{nm} \mathrm{Bi} \mathrm{Te}_{3}$. The figure of merit of nanostructured $\mathrm{Bi}_{1.71} \mathrm{Co}_{0.22} \mathrm{Te}_{1.71}$ alloy was found to increase by $40 \%$ to a $Z T \sim 1.4 \times 10^{-4}$ at room temperature. Although overall the figure of merit is low, the effect of Co alloying and hydrazine treatment shows potential as a route to optimise the figure of merit.

A potential novel material for thermoelectrics applications is inorganicorganic perovskite single crystals. Here we report a synthetic strategy to successfully grow large millimetre scale single crystals of $\mathrm{MAPbBr}_{3-x} \mathrm{Cl}_{x}$, $\mathrm{FAPbBr}_{3-\mathrm{x}} \mathrm{Cl}_{\mathrm{x}}$, and $\mathrm{MAPb}_{1-\mathrm{x}} \mathrm{Sn}_{\mathrm{x}} \mathrm{Br}_{3}$ (MA = methylammonium and $\mathrm{FA}=$ formamidinium) using inverse temperature crystallisation (ITC) in a matter of days. This is the first reported case of mixed $\mathrm{Br} / \mathrm{Cl}$ single crystals with a FA cation and mixed $\mathrm{Pb} / \mathrm{Sn}$ based perovskites grown using ITC. The bandgap of these single crystals was successfully tuned by altering the halide and 
metal site composition. It was found that single crystals of $\mathrm{FAPbBr}_{3-\mathrm{x}} \mathrm{Cl}_{\mathrm{x}}$ were prone to surface degradation with increased synthesis time. This surface degradation was observed to be reversible by placing the single crystals in an antisolvent such as chloroform.

A tentative model was proposed to analyse the IV characteristics of the single crystal perovskites in order to extract mobilities and diffusion lengths. The $\mathrm{MAPbBr}_{3}$ and $\mathrm{MAPbBr}_{2.5} \mathrm{Cl}_{0.5}$ single crystal mobilities were found to be between $30-390 \mathrm{~cm}^{2} \mathrm{~V}^{-1} \mathrm{~s}^{-1}$ and $10-100 \mathrm{~cm}^{2} \mathrm{~V}^{-1} \mathrm{~s}^{-1}$ respectively, the diffusion lengths were found to be between $2-8 \mu \mathrm{m}$ and $1-4 \mu \mathrm{m}$ respectively. This is an improvement over polycrystalline thin film perovskites and comparable to other single crystal perovskites. The conductance of $\mathrm{MAPb}_{1-\mathrm{x}} \mathrm{Sn}_{\mathrm{x}} \mathrm{Br}_{3}$ based perovskites was found to increase by 2 orders of magnitude even with just $1 \%$ of $\mathrm{Sn}$ incorporated. The thermal conductivity of $\mathrm{MAPbBr}_{3}$ single crystals was found to be $\sim 1.12 \mathrm{~W} \mathrm{~m}^{-1} \mathrm{~K}^{-1}$ at room temperature which is reasonable low for single crystals, however no other thermoelectric properties could be measured due to the self cleaving nature of the single crystals with decreasing temperature and the high resistivity of the material. 




\section{Acknowledgements}

I would like express my deep gratitude to Dr Jonathan Halpert, my supervisor for his patient guidance, helpful insight, useful critiques and encouragement, and advice on science and life in general.

I would like to acknowledge Dr Shen Chong at the Robinson Research Institute for all his help with preparing and measuring samples using the PPMS.

I would like to thank Dr Holger Fiedler for all his help with the electrical characterization measurements and his discussions with interpreting the results.

I would like to acknowledge David Flynn for all his help with training on the electron microscopes and answering any queries I had.

I would like to thank Dr Brendan Darby for his help measuring the absorption spectra of my highly scattering samples.

I would like to thank Parth Vashishtha and the rest of the Halpert group for all their help and support in the lab.

Lastly I would like to thank all my friends and family for all their support and encouragement throughout all my endeavours. 



\section{Contents}

$\begin{array}{ll}\text { Abstract } & \text { iii }\end{array}$

Acknowledgements vii

1 Introduction 1

1.1 Thermoelectric materials . . . . . . . . . . . . . . 2

1.1.1 Thermoelectric effect .............. 2

1.1.2 Transport properties .............. 3

1.1.3 Thermoelectric device . . . . . . . . . . . . 6

1.1.4 Efficiency and the figure of merit . . . . . . . . 6

1.1.4.1 Figure of merit .......... 8

1.1.5 Increasing the figure of merit . . . . . . . . . 8

1.1.5.1 Advances in thermoelectric materials . . . . 10

1.1.6 Nanostructured thermoelectric materials . . . . . . . 11

1.1.6.1 Nanocomposite polycrystalline materials . . 12

1.1.7 $\mathrm{Bi}_{2} \mathrm{Te}_{3}$ nanomaterials . . . . . . . . . . . 13

1.1.8 Alternative materials . . . . . . . . . . . . 13

1.2 Organic-inorganic single crystal perovskites . . . . . . . . . . . 14

1.3 In this thesis . . . . . . . . . . . . . . . . . 15

2 Materials and Methods $\quad 17$

2.1 Characterisation techniques ............. 17

2.1.1 Transmission electron microscopy . . . . . . . . . . . . 17

2.1.2 Scanning electron microscopy . . . . . . . . . . . 18

2.1.3 Powder X-ray diffraction . . . . . . . . . . . . . . 18

2.1.4 Fourier transform infrared spectroscopy . . . . . . . . 18

2.1.5 Optical characterisation .............. 18

2.2 Thermoelectric measurements . . . . . . . . . . . . . . . . . 19

2.2.1 Thermal conductivity ............. 19

2.2.2 Seebeck coefficient .............. 20

2.2 .3 Electrical resistivity ............... . . 20

2.2.4 Software modelling .............. 20 
2.2.5 Sample preparation. . . . . . . . . . . . . . 21

2.2.5.1 Nanocomposite pellets . . . . . . . . . . 21

2.2.5.2 Mounting samples for PPMS . . . . . . . . . 21

3 Synthesis and characterisation of nanostructured $\mathrm{Bi}_{2} \mathrm{Te}_{3}$

3.1 Experimental . . . . . . . . . . . . . . . . 24

3.1.1 Materials . . . . . . . . . . . . . . . . . . . . 24

3.2 Initial $\mathrm{Bi}_{2} \mathrm{Te}_{3}$ synthesis attempts . . . . . . . . . . . . 25

3.2.1 Synthesis via bismuth(III) oleate . . . . . . . . . . 25

3.2.1.1 Characterisation and thermoelectric properties 25

3.2.2 Synthesis with different capping ligands . . . . . . . 30

3.2.2.1 Characterisation and thermoelectric properties 31

3.2.3 Ligand exchange . . . . . . . . . . . . . . . 35

3.3 Nanostructured $\mathrm{Bi}_{2} \mathrm{Te}_{3}$ Synthesized via Bismuth Nanoparticles 36

3.3.1 Synthesis via Bi nanoparticle . . . . . . . . . . . . 36

3.3.1.1 Characterisation and thermoelectric properties 38

3.3.2 Doped $\mathrm{Bi}_{2} \mathrm{Te}_{3}$ nanostructures . . . . . . . . . . 45

3.3.2.1 Characterisation and thermoelectric properties 46

3.4 Brief investigation of alternative nanoparticles . . . . . . . 57

3.4 .1 SnSe nanoparticles . . . . . . . . . . . . . . . 57

3.4.1.1 Synthesis . . . . . . . . . . . . . . . 57

3.4.1.2 Results . . . . . . . . . . . . . . . 59

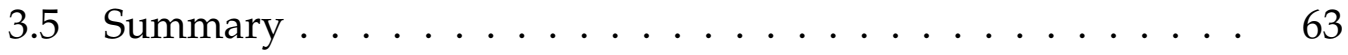

4 Synthesis and characterisation of perovskite single crystals 65

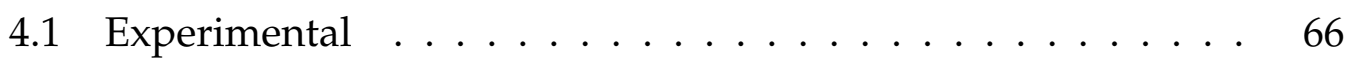

4.1 .1 Materials . . . . . . . . . . . . . . . . . . . . 66

$4.2 \mathrm{MAPbBr}_{3-x} \mathrm{Cl}_{\mathrm{x}}$ based perovskites $\ldots \ldots \ldots \ldots \ldots \ldots 66$

4.2 .1 Synthesis . . . . . . . . . . . . . . 66

4.2.2 Results and discussion . . . . . . . . . . . . . . 68

4.2.2.1 Electrical characterisation and modelling . . . 72

$4.3 \mathrm{FAPbBr}_{3-\mathrm{x}} \mathrm{Cl}_{\mathrm{x}}$ based perovskites $\ldots \ldots \ldots \ldots \ldots \ldots$

4.3 .1 Synthesis . . . . . . . . . . . . . . . . 80

4.3.2 Results and discussion . . . . . . . . . . . . . . . 81

4.3.2.1 Electrical characterisation . . . . . . . . . . . 87

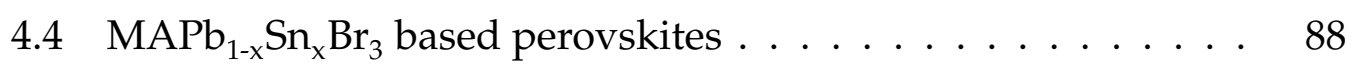

4.4 .1 Synthesis . . . . . . . . . . . . . . . . . 88

4.4 .2 Results and discussion . . . . . . . . . . . . . . . . . . 89

4.5 Thermoelectric properties . . . . . . . . . . . . . 93 
4.6 Summary ............................ 95

5 Conclusion and future work $\quad 97$

5.1 Conclusion . . . . . . . . . . . . . . . . 97

5.2 Future work ......................... 99

$\begin{array}{ll}\text { Bibliography } & 101\end{array}$ 



\section{List of Figures}

1.1 Schematic of a thermocouple . . . . . . . . . . . 2

1.2 Schematic of a basic thermoelectric device . . . . . . . . . 6

1.3 Optimising thermoelectric properties with carrier concentration 9

1.4 Perovskite crystal structure $\ldots \ldots \ldots \ldots$

2.1 JEOL JEM2100F TEM . . . . . . . . . . . . . . . . . . . 17

2.2 Physical Property Measurement System . . . . . . . . . . . . 19

2.3 PPMS sample mounting configuration . . . . . . . . . . . . 21

3.1 Powder XRD spectra of $\mathrm{Bi}_{2} \mathrm{Te}_{3}$ synthesized at $50^{\circ} \mathrm{C}$ and $100{ }^{\circ} \mathrm{C}$ via bismuth(III) oleate . . . . . . . . . . . . . . 26

3.2 TEM micrographs of $\mathrm{Bi}_{2} \mathrm{Te}_{3}$ synthesised at $50^{\circ} \mathrm{C}$ via bismuth(III) oleate . . . . . . . . . . . . . . . . . 26

3.3 TEM micrographs of $\mathrm{Bi}_{2} \mathrm{Te}_{3}$ synthesised at $100{ }^{\circ} \mathrm{C}$ via bismuth(III) oleate . . . . . . . . . . . . . . . . . . . 28

3.4 Thermoelectric properties of $\mathrm{Bi}_{2} \mathrm{Te}_{3}$ synthesized at 50 and 100 ${ }^{\circ} \mathrm{C}$ via bismuth(III) oleate . . . . . . . . . . . . . . . . . . . . . . 29

3.5 Powder XRD spectra of $\mathrm{Bi}_{2} \mathrm{Te}_{3}$ capped with OLA, DDT, and TGA 31

3.6 TEM micrographs of $\mathrm{Bi}_{2} \mathrm{Te}_{3}$ capped with DDT $\ldots \ldots \ldots . \quad 32$

3.7 TEM micrographs of $\mathrm{Bi}_{2} \mathrm{Te}_{3}$ capped with OLA $\ldots \ldots \ldots$

3.8 TEM micrographs of $\mathrm{Bi}_{2} \mathrm{Te}_{3}$ capped with TGA $\ldots \ldots \ldots$

3.9 Thermoelectric properties of $\mathrm{Bi}_{2} \mathrm{Te}_{3}$ capped with OLA and TGA 34

3.10 Hydrazine and sulfide ligand exchange . . . . . . . . . 35

3.11 Powder XRD spectra of $\mathrm{Bi}_{2} \mathrm{Te}_{3}$ synthesised at $60^{\circ} \mathrm{C}$ and $120^{\circ} \mathrm{C}$ via $B i$ nanoparticles . . . . . . . . . . . . . . 38

3.12 TEM micrographs and particle Distribution of bismuth nanoparticle precursor . . . . . . . . . . . . . . . . . . . 39

3.13 TEM micrographs and particle distribution of $\mathrm{Bi}_{2} \mathrm{Te}_{3}$ synthesised at $60^{\circ} \mathrm{C}$ via bismuth nanoparticles . . . . . . . . . . 39

3.14 STEM/EDS elemental map of $\mathrm{Bi}_{2} \mathrm{Te}_{3}$ synthesised at $60^{\circ} \mathrm{C}$ via bismuth nanoparticles . . . . . . . . . . . . . . . . . 40 
3.15 SEM/EDS elemental map of nanocomposite pellet prepared from $\mathrm{Bi}_{2} \mathrm{Te}_{3}$ synthesised at $60{ }^{\circ} \mathrm{C}$ via bismuth nanoparticles . .

3.16 Thermoelectric properties of as-synthesized and hydrazine capped $\mathrm{Bi}_{2} \mathrm{Te}_{3}$ synthesized at $60^{\circ} \mathrm{C}$ via bismuth nanoparticles

3.17 TEM micrographs and particle distribution of $\mathrm{Bi}_{2} \mathrm{Te}_{3}$ synthesised at $120{ }^{\circ} \mathrm{C}$ via bismuth nanoparticles . . . . . . . . . .

3.18 STEM/EDS elemental map of $\mathrm{Bi}_{2} \mathrm{Te}_{3}$ synthesised at $120^{\circ} \mathrm{C}$ via bismuth nanoparticles . . . . . . . . . . .

3.19 SEM/EDS elemental map of nanocomposite pellet prepared from $\mathrm{Bi}_{2} \mathrm{Te}_{3}$ synthesised at $120{ }^{\circ} \mathrm{C}$ via bismuth nanoparticles .

3.20 Thermoelectric properties of as-synthesized and hydrazine capped $\mathrm{Bi}_{2} \mathrm{Te}_{3}$ synthesized at $120{ }^{\circ} \mathrm{C}$ via bismuth nanoparti-

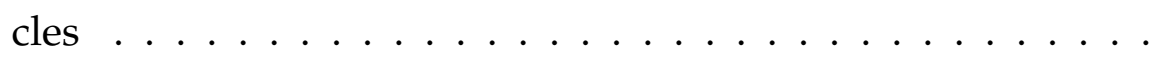

3.21 Powder XRD spectra of doped $\mathrm{Bi}_{2} \mathrm{Te}_{3}$ synthesised at $120{ }^{\circ} \mathrm{C}$ via Bi nanoparticles . . . . . . . . . . . . . 46

3.22 TEM micrographs and particle distribution of $\mathrm{Cu}$ doped bismuth nanoparticle precursor . . . . . . . . . . . . .

3.23 TEM micrographs and particle distribution of $\mathrm{Cu}$ doped $\mathrm{Bi}_{2} \mathrm{Te}_{3}$ synthesised at $120^{\circ} \mathrm{C}$ via bismuth nanoparticles . . . . . . .

3.24 STEM/EDS elemental map of $\mathrm{Cu}$ doped $\mathrm{Bi}_{2} \mathrm{Te}_{3}$ synthesised at $120{ }^{\circ} \mathrm{C}$ via bismuth nanoparticles . . . . . . . . . . .

3.25 SEM/EDS elemental map of nanocomposite pellet prepared from $\mathrm{Cu}$ doped $\mathrm{Bi}_{2} \mathrm{Te}_{3}$ synthesised at $120^{\circ} \mathrm{C}$ via bismuth nanopar-

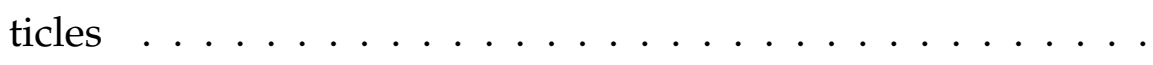

3.26 TEM micrographs and particle distribution of Ni doped bismuth nanoparticle precursor . . . . . . . . . . 50

3.27 TEM micrographs of $\mathrm{Ni}$ doped $\mathrm{Bi}_{2} \mathrm{Te}_{3}$ synthesised at $120^{\circ} \mathrm{C}$ via bismuth nanoparticles . . . . . . . . . . . . .

3.28 SEM/EDS elemental map of nanocomposite pellet prepared from $\mathrm{Ni}$ doped $\mathrm{Bi}_{2} \mathrm{Te}_{3}$ synthesised at $120^{\circ} \mathrm{C}$ via bismuth nanopar-

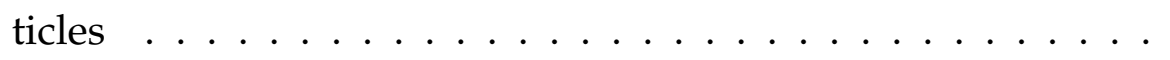

3.29 TEM micrographs and particle distribution of Co doped bismuth nanoparticle precursor . . . . . . . . . . . 52

3.30 TEM micrographs of Co doped $\mathrm{Bi}_{2} \mathrm{Te}_{3}$ synthesised at $120^{\circ} \mathrm{C}$ via bismuth nanoparticles . . . . . . . . . . .

3.31 SEM/EDS elemental map of nanocomposite pellet prepared from $\mathrm{Co}$ doped $\mathrm{Bi}_{2} \mathrm{Te}_{3}$ synthesised at $120^{\circ} \mathrm{C}$ via bismuth nanopar-

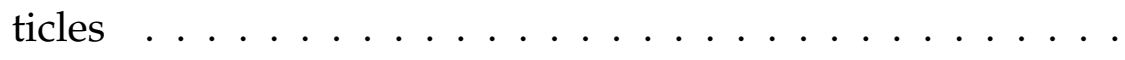


3.32 Thermoelectric properties of doped/alloyed $\mathrm{Bi}_{2} \mathrm{Te}_{3}$ nanoparticles 55

3.33 Powder XRD spectra of SnSe nanoparticles . . . . . . . . . . 59

3.34 TEM micrographs of SnSe(TS1) nanoparticles . . . . . . . . . 60

3.35 STEM/EDS elemental map of SnSe(TS1) nanoparticles . . . . 60

3.36 TEM micrographs of SnSe(TS2) nanoparticles . . . . . . . . . . 60

3.37 STEM/EDS elemental map of SnSe(TS2) nanoparticles . . . . 60

3.38 Quality of $\mathrm{Sn}$ precursor after degassing . . . . . . . . . . 61

3.39 TEM micrographs of SnSe(TS3) nanoparticles . . . . . . . . . . 62

3.40 STEM/EDS elemental map of SnSe(TS3) nanoparticles . . . . 62

$4.1 \mathrm{MAPbBr}_{3}$ crystal growth at different time intervals . . . . . . 67

4.2 Photographs and powder XRD spectra of $\mathrm{MAPbBr}_{3-x} \mathrm{Cl}_{x}$ perovskites............................. 69

4.3 SEM-SEI images of $\mathrm{MAPbBr}_{3-x} \mathrm{Cl}_{x}$ perovskites . . . . . . . 70

4.4 Steady-state absorption, photoluminescence emission and Tauc plots of $\mathrm{MAPbBr}_{3-x} \mathrm{Cl}_{x}$ perovskites . . . . . . . . . . 71

4.5 Bandgap of $\mathrm{MAPbBr}_{3-x} \mathrm{Cl}_{x}$ with respect to $\mathrm{Cl}$ inclusion and $\mathrm{PL}$ time decay traces on $\mathrm{MAPbBr}_{3-x} \mathrm{Cl}_{x}$ perovskites . . . . . . 71

4.6 IV trace ssing sandwich geometry for $\mathrm{MAPbBr}_{3} \ldots \ldots . . . . .74$

4.7 Device structure used to measure IV characteristics and white light LED emission spectrum . . . . . . . . . . . . 75

4.8 IV trace for $\mathrm{MAPbBr}_{3-x} \mathrm{Cl}_{x}$ perovskites . . . . . . . . . 75

4.9 IV trace for $\mathrm{MAPbBr}_{3-x} \mathrm{Cl}_{x}$ perovskites in a dark environment and under white light illumination . . . . . . . . . . 79

$4.10 \mathrm{On} /$ off ratio and comparison with white light LED emission spectrum for $\mathrm{MAPbBr}_{3-x} \mathrm{Cl}_{x}$ perovskites . . . . . . . . . 80

4.11 Photographs of $\mathrm{FAPbBr}_{3-x} \mathrm{Cl}_{x}$ perovskites with a total synthesis time of 20 hours and $>40$ hours . . . . . . . . . . 81

4.12 Powder XRD spectra and photographs of ground $\mathrm{FAPbBr}_{3-x} \mathrm{Cl}_{x}$ perovskites with a total synthesis time of 20 hours and $>40$ hours 82

$4.13 \mathrm{SEM}-\mathrm{SEI}$ images of $\mathrm{FAPbBr}_{3-x} \mathrm{Cl}_{x}$ perovskites . . . . . . . . 83

4.14 UV-vis absorption, PL emission, and corresponding Tauc plots for $\mathrm{FAPbBr}_{3-x} \mathrm{Cl}_{x}$ perovskites . . . . . . . . . . . 84

4.15 Surface repair of degraded $\mathrm{FAPbBr}_{3-x} \mathrm{Cl}_{x}$ single crystals using antisolvents ...................... 85

4.16 PL emission of degraded crystal before and after 4 weeks in chloroform ........................ 85

4.17 Bandgap of $\mathrm{FAPbBr}_{3-x} \mathrm{Cl}_{x}$ with respect to $\mathrm{Cl}$ inclusion and $\mathrm{PL}$ time decay traces on $\mathrm{FAPbBr}_{3-x} \mathrm{Cl}_{x}$ perovskites . . . . . . 86 
$4.18 \mathrm{IV}$ trace for $\mathrm{FAPbBr}_{3-x} \mathrm{Cl}_{x}$ perovskites $\ldots \ldots \ldots \ldots \ldots 87$

$4.19 \mathrm{On} /$ off ratio and comparison with white light LED emission spectrum for $\mathrm{FAPbBr}_{3-x} \mathrm{Cl}_{x}$ perovskites $\ldots \ldots \ldots \ldots$. . . 87

4.20 Photographs and powder XRD spectra of $\mathrm{MAPb}_{1-x} \mathrm{Sn}_{x} \mathrm{Br}_{3}$ per-

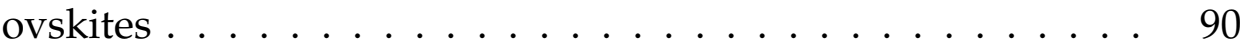

4.21 SEM-SEI images of $\mathrm{MAPb}_{1-x} \mathrm{Sn}_{x} \mathrm{Br}_{3}$ perovskites . . . . . . 91

4.22 UV-vis absorption and corresponding Tauc plots for $\mathrm{MAPb}_{1-x} \mathrm{Sn}_{x} \mathrm{Br}_{3}$

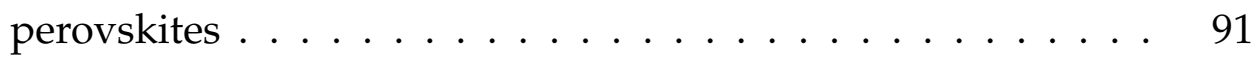

4.23 Bandgap of $\mathrm{MAPb}_{1-x} \mathrm{Sn}_{x} \mathrm{Br}_{3}$ with respect to Sn inclusion . . . 92

$4.24 \mathrm{IV}$ trace for $\mathrm{MAPb}_{1-x} \mathrm{Sn}_{x} \mathrm{Br}_{3}$ perovskites . . . . . . . . . 92

$4.25 \mathrm{On}$ /off ratio for $\mathrm{MAPb}_{1-x} \mathrm{Sn}_{x} \mathrm{Br}_{3}$ perovskites . . . . . . . 93

4.26 $\mathrm{MAPbBr}_{3}$ mounted for PPMS and thermal conductivity of $\mathrm{MAPbBr}_{3} \ldots \ldots \ldots \ldots \ldots \ldots \ldots \ldots \ldots$ 


\section{List of Tables}

3.1 Composition determined by TEM/EDS for $\mathrm{Bi}_{2} \mathrm{Te}_{3}$ synthesized at $50{ }^{\circ} \mathrm{C}$ via bismuth(III) oleate . . . . . . . . . . . . .

3.2 Composition determined by TEM/EDS for $\mathrm{Bi}_{2} \mathrm{Te}_{3}$ synthesized at $100{ }^{\circ} \mathrm{C}$ via bismuth(III) oleate . . . . . . . . . . . . . 28

3.3 Composition determined by TEM/EDS for $\mathrm{Bi}_{2} \mathrm{Te}_{3}$ capped with DDT, OLA, and TGA . . . . . . . . . . . . . .

3.4 Conductivity of nanocomposite $\mathrm{Bi}_{2} \mathrm{Te}_{3}$ pellets before and after Ligand exchange treatment . . . . . . . . . . .

3.5 Composition determined by STEM/EDS for nanocomposite pellet of $\mathrm{Bi}_{2} \mathrm{Te}_{3}$ synthesised at $60{ }^{\circ} \mathrm{C}$ via bismuth nanoparticles

3.6 Composition determined by SEM/EDS for nanocomposite pellet of $\mathrm{Bi}_{2} \mathrm{Te}_{3}$ synthesised at $120{ }^{\circ} \mathrm{C}$ via bismuth nanoparticles .

3.7 Composition determined by SEM/EDS for nanocomposite pellet of $\mathrm{Cu}$ doped $\mathrm{Bi}_{2} \mathrm{Te}_{3}$ synthesised at $120^{\circ} \mathrm{C}$ via bismuth nanopar-

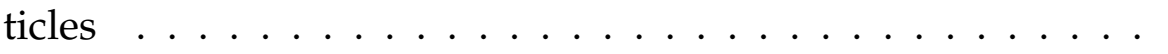

3.8 Composition determined by SEM/EDS for nanocomposite pellet of $\mathrm{Ni}$ doped $\mathrm{Bi}_{2} \mathrm{Te}_{3}$ synthesised at $120^{\circ} \mathrm{C}$ via bismuth nanopar-

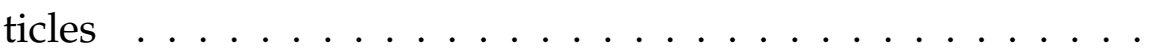

3.9 Composition determined by TEM/EDS for Co doped bismuth nanoparticle precursor . . . . . . . . . . .

3.10 Composition determined by SEM/EDS for nanocomposite pellet of $\mathrm{Co}$ doped $\mathrm{Bi}_{2} \mathrm{Te}_{3}$ synthesised at $120^{\circ} \mathrm{C}$ via bismuth nanopar-

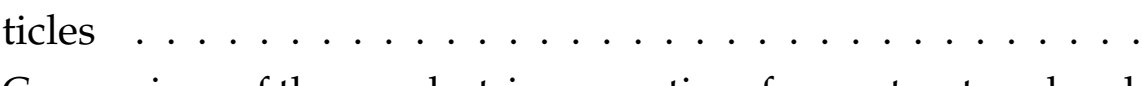

3.11 Comparison of thermoelectric properties of nanostructured and bulk $\mathrm{Bi}_{2} \mathrm{Te}_{3}$ samples at $300 \mathrm{~K} \ldots \ldots$. . . . . . . . . . 56

3.12 Composition determined by TEM/EDS for SnSe nanoparticles 61

4.1 $\mathrm{Br} / \mathrm{Cl}$ ratio determined from SEM/EDS, lattice constant determined from $\mathrm{XRD}$, and band gap calculated from Tauc Plots for $\mathrm{MAPbBr}_{3-x} \mathrm{Cl}_{x}$ perovskites ............. 70

4.2 PL decay lifetimes for $\mathrm{MAPbBr}_{3-x} \mathrm{Cl}_{x}$ perovskites . . . . . . 71 
4.3 Extracted mobilities and diffusion lengths of $\mathrm{MAPbBr}_{3-x} \mathrm{Cl}_{x}$

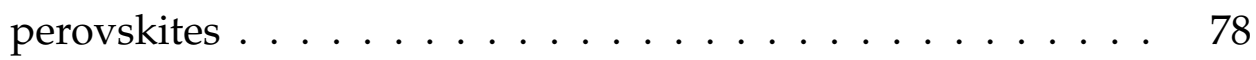

$4.4 \mathrm{On}$ /off ratio for $\mathrm{MAPbBr}_{3-x} \mathrm{Cl}_{x} \ldots \ldots \ldots \ldots \ldots \ldots$

$4.5 \mathrm{Br} / \mathrm{Cl}$ ratio determined from SEM/EDS, lattice constant determined from $\mathrm{XRD}$, and bandgap calculated from Tauc plots for $\mathrm{FAPbBr}_{3-x} \mathrm{Cl}_{x}$ perovskites $\ldots \ldots \ldots \ldots \ldots \ldots$

4.6 PL decay lifetimes for $\mathrm{FAPbBr}_{3-x} \mathrm{Cl}_{x}$ perovskites $\ldots \ldots \ldots 86$

$4.7 \mathrm{On}$ /off ratio for $\mathrm{FAPbBr}_{3-x} \mathrm{Cl}_{x} \ldots \ldots \ldots \ldots \ldots$

$4.8 \mathrm{~Pb} / \mathrm{Sn}$ ratio determined from SEM/EDS, lattice constant determined from $\mathrm{XRD}$, and bandgap calculated from Tauc plots for

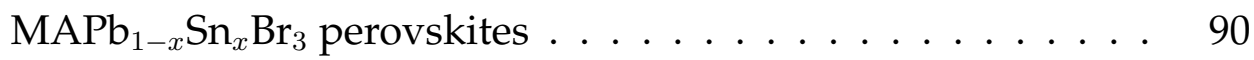

$4.9 \mathrm{On}$ /off ratio for $\mathrm{MAPb}_{1-x} \mathrm{Sn}_{x} \mathrm{Br}_{3} \ldots \ldots \ldots \ldots \ldots$

4.10 Conductance for MA based perovskites . . . . . . . . . 94 


\title{
List of Abbreviations
}

\author{
0D Zero Dimensional \\ ATR Attenuated Total Reflection \\ CIP Cold Isostatic Pressing \\ CuAcAc Copper(II) Acetylacetonate \\ DCM Dichloromethane \\ DDT 1-Dodecanethiol \\ DMF N,N-Dimethylformamide \\ DMSO Dimethyl Sulfoxide \\ DOS Density of States \\ EDS Energy Dispersive X-ray Spectroscopy \\ EMF Electromotive Force \\ FA Formamidinium \\ FABr Formamidinium Bromide \\ FTIR Fourier Transform Infrared \\ FWHM Full Width at Half Maximum \\ GBL $\quad \gamma$-Butyrolactone \\ ICDD International Centre for Diffraction Data \\ IPA Isopropanol \\ ITC Inverse Temperature Crystallisation \\ IV Current Voltage \\ LED Light Emitting Diode \\ MA Methylammonium \\ MABr Methylammonium Bromide \\ MACl Methylammonium Chloride \\ OA Oleic Acid \\ ODPA Octadecylphosphonic Acid \\ OLA Oleylamine \\ PL Photoluminescence \\ PPMS Physical Property Measurement System \\ PTFE Polytetrafluoroethylene \\ RPM Revolutions Per Minute \\ SCLC Space Charge Limited Current
}


SEI Secondary Electron Image

SEM Scanning Electron Microscope

STEG Solar Thermoelectric Generators

STEM Scanning Tunnelling Electron Microscope

TCSPC Time Correlated Single Photon Counting

TE Thermoelectric

TEG Thermoelectric Generator

TEM Transmission Electron Microscope

THF Tetrahydrofuran

TOP Trioctylphosphine

VAC Antisolvent Vapor-Assisted Crystallisation

XRD X-Ray Diffraction

ZT Dimensionless Figure of Merit 


\section{Physical Constants}

\begin{tabular}{|c|c|}
\hline Boltzmann constant & $k_{B}=1.38064852 \times 10^{-23} \mathrm{JK}^{-1}$ \\
\hline Elementary charge & $q=1.6021766208 \times 10^{-19} \mathrm{C}$ \\
\hline Gravity & $g=9.80665 \mathrm{~m} \mathrm{~s}^{-2}$ \\
\hline Permittivity of free space & $\epsilon_{0}=8.854187817 \times 10^{-12} \mathrm{~F} \mathrm{~m}^{-}$ \\
\hline
\end{tabular}





\section{List of Symbols}

\begin{tabular}{|c|c|c|}
\hline$V$ & voltage & $\mathrm{V}$ \\
\hline$S$ & Seebeck coefficient (thermopower) & $\mathrm{V} \mathrm{K}^{-1}$ \\
\hline$T$ & temperature & $\mathrm{K}$ \\
\hline$Q$ & heat flux & $\mathrm{W}$ \\
\hline$I$ & current & $\mathrm{A}$ \\
\hline$K$ & thermal conductance & $\mathrm{WK}^{-1}$ \\
\hline$R$ & resistance & $\Omega$ \\
\hline$\Delta T$ & temperature difference & K \\
\hline$Z$ & figure of merit & $\mathrm{K}^{-1}$ \\
\hline$Z T$ & dimensionless figure of merit & \\
\hline$n$ & carrier concentration & $\mathrm{m}^{-3}$ \\
\hline$m^{*}$ & effective mass & $\mathrm{kg}$ \\
\hline$E_{F}$ & Fermi energy & $\mathrm{J}$ \\
\hline$L$ & length & $\mathrm{m}$ \\
\hline$A$ & area & $\mathrm{m}^{2}$ \\
\hline$l$ & channel length & $\mathrm{m}$ \\
\hline$J$ & current density & $\mathrm{A} \mathrm{m}^{-2}$ \\
\hline$V_{\mathrm{TFL}}$ & trap free limited voltage & $\mathrm{V}$ \\
\hline$N_{t}$ & trap density & $\mathrm{m}^{-3}$ \\
\hline$t$ & thickness & $\mathrm{m}$ \\
\hline$E$ & electric field & $\mathrm{Vm}^{-1}$ \\
\hline$G$ & conductance & $\Omega^{-1}$ \\
\hline$V_{I}$ & intersection voltage & $\mathrm{V}$ \\
\hline$\Pi$ & Peltier coefficient & $\mathrm{V}$ \\
\hline$\eta$ & efficiency & \\
\hline$\sigma$ & electrical conductivity & $\mathrm{Sm}^{-1}$ \\
\hline$\kappa$ & thermal conductivity & 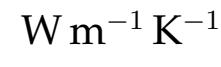 \\
\hline$\mu$ & mobility & $\mathrm{m}^{2} \mathrm{~V}^{-1} \mathrm{~s}^{-1}$ \\
\hline$\epsilon_{r}$ & relative permittivity & \\
\hline$\Theta$ & trapping factor & \\
\hline$\mu_{R}$ & recombination mobility & $\mathrm{m}^{2} \mathrm{~V}^{-1} \mathrm{~s}^{-1}$ \\
\hline
\end{tabular}


xxiv

$\tau$ recombination lifetime

$\mathrm{S}$ 


\section{Chapter 1}

\section{Introduction}

Energy consumption worldwide is constantly increasing, bringing with it the demand for low cost, environmentally friendly, and efficient energy technologies. A large fraction of the generated energy is lost in the form of heat, where in the United States total waste heat is more than $60 \%$ of the input energy ${ }^{1}$. If a fraction of this waste heat could be recovered then it could have a significant impact on our overall energy production and consumption. A potential method to harness this waste heat energy is to use thermoelectric energy conversion in which heat is converted into electrical energy.

Thermoelectric devices have no moving parts which makes them more reliable and lower maintenance compared with traditional mechanical energy conversion systems. However due to low energy conversion efficiencies and high manufacturing costs, thermoelectric devices are still not widely used except in niche environments like radioactive isotopic thermoelectric generators (TEG) for deep space probes and miniature coolers for opto-electronic devices $^{1,2}$. If efficiencies greater then $15 \%$ are achieved for thermoelectric devices than this could have a significant impact on overall energy utilization and its commercial viability.

Before the past decade increases in thermoelectric efficiency had become rather stagnant however thanks to the increased research into thermoelectrics in the last decade, we have seen a significant increase in the thermoelectric efficiencies, almost doubling bringing with it a resurgence of commercial interests. Some of these increases in efficiencies have come from developments in nanostructural engineering in thermoelectric materials ${ }^{3-6}$, band engineering in $\mathrm{Pb}$ chalcogenides ${ }^{7,8}$, complex crystal structures ${ }^{9-11}$, and anharmonicity in $\mathrm{SnSe} \mathrm{1}^{12,13}$.

Materials such as $\mathrm{Bi}_{2} \mathrm{Te}_{3}$ and $\mathrm{SnSe}$ have already shown great potential as thermoelectric materials. In this thesis we will aim to use nanostructuring and doping to further improve the thermoelectric properties of $\mathrm{Bi}_{2} \mathrm{Te}_{3}$. We 
will also look at inorganic-organic single crystal perovskites as a novel thermoelectric materials. These materials have already seen rapid growth in solar cell efficiencies and has been computational predicted to have a high $Z T$ at room temperature ${ }^{14-18}$.

\subsection{Thermoelectric materials}

All materials exhibit thermoelectric effects in which a thermal difference creates an electric potential or vice versa. The term thermoelectric materials is reserved for materials which are good at converting between heat and electrical energy.

\subsubsection{Thermoelectric effect}

A thermoelectric effect refers to any phenomena which involves the exchange of heat and electrical potential energy. This is typically described by three different phenomena. A thermocouple, shown in Figure 1.1, which can be considered as a conducting loop consisting of two different materials A and B can be used to conveniently discuss thermoelectric effects. The first of these

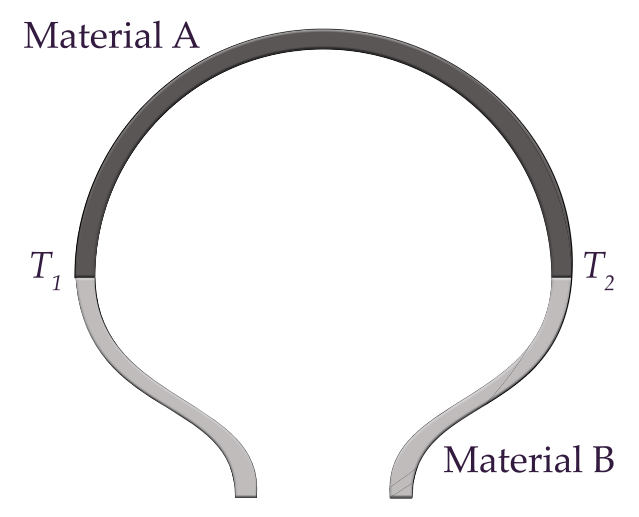

FIGURE 1.1: Schematic for a thermocouple consisting of two dissimilar materials A and B.

thermoelectric effects was discovered in 1821 by Thomas Johann Seebeck ${ }^{19}$. In his experiment he found that if the two junctions between materials $\mathrm{A}$ and $B$ in Figure 1.1 are at different temperatures $\left(T_{1} \neq T_{2}\right)$ then an electromotive force (emf) would be produced. This is known as the Seebeck effect. In general the potential difference produced by a temperature gradient can be given by:

$$
\Delta V=\left(S_{A}-S_{B}\right) \Delta T
$$


where $S$ is defined as the Seebeck coefficient (or thermopower) and is an intrinsic property of a material. Consequently we can't actually measure the absolute Seebeck coefficient of a homogeneous conductor as the resulting emf across it will be zero. In order to practically measure the Seebeck coefficient of a material we must form a thermocouple with a reference material with a known or negligibly small Seebeck coefficient ${ }^{20}$.

The complementary of this effect was later discovered in 1834 by Jean Peltier $^{21}$. He determined that if an electrical circuit is connected to the legs of the thermocouple and a current is passed through the loop then heat can be produced or absorbed at the junctions between the two materials. This is known as the Peltier effect. In this case the rate of heat generated or removed at the junction is given by:

$$
Q=\left(\Pi_{A}-\Pi_{B}\right) I
$$

where $\Pi$ is the Peltier coefficient. If $\Pi_{A}>\Pi_{B}$ then $T_{1}$ becomes colder and $T_{2}$ becomes warmer in the thermocouple allowing for easily controlled cooling ${ }^{22}$.

The last phenomena is known as the Thomson effect, which was discovered by William Thomson in $1851^{23}$. He predicted that if a material has a temperature gradient with a simultaneous flow of current then heat will be produced or absorbed by the conductor.

\subsubsection{Transport properties}

Transport phenomena are described by two characteristic mechanisms: the driving force of the external fields and the dissipative effect of the scattering of the carriers by phonons and defects ${ }^{1,22}$. If we consider the effects of these two mechanisms on the distribution function (which describes the probability of an energy level being occupied by a particle) then we can analyse the transport properties in a crystalline material. The interplay between these two contributions is described by the Boltzmann equation ${ }^{22}$. In the absence of external fields, under thermal equilibrium, and homogeneous temperature conditions the distribution function is given by the Fermi distribution:

$$
f_{0}=\frac{1}{\exp \left(\frac{E-\mu}{k_{B} T}\right)+1} \text {. }
$$

If we consider the change in the distribution function away from equilibrium during the time $t-d t$ to $t$, under an applied external field then the distribution 
can be given by:

$$
f(\boldsymbol{r}, \boldsymbol{k}, t)=f(\boldsymbol{r}-\boldsymbol{v} d t, \boldsymbol{k}+e \mathscr{E} d t / \hbar, t-d t)
$$

where $k$ is the quantum number for the periodic crystal. Expanding this up to terms linear in $d t$ and adding the additional term to account for scattering events gives us the Boltzmann equation:

$$
\left(\frac{\partial f}{\partial t}\right)_{s}=\boldsymbol{v} \cdot \nabla_{r} f-\frac{\boldsymbol{F}}{\hbar} \cdot \nabla_{k} f .
$$

The generalized Boltzmann equation (1.5) is complicated and difficult to solve. The scattering term is often modelled using the concept of relaxation time in order to simplify this, which is given by:

$$
\left(\frac{\partial f}{\partial t}\right)_{s}=\frac{f-f_{0}}{\tau}
$$

where $\tau$ is the relaxation time, and we assume that the rate at which $f$ returns to the equilibrium distribution $f_{0}$ due to scattering is proportional to its deviation of $f$ from $f_{0}$. The assumption behind the relaxation time model is that scattering merely serves to drive a non-equilibrium distribution back towards thermal equilibrium. Using the assumption that the local deviation from equilibrium is small and the concept of a relaxation time the Boltzmann equation can be linearised to give an approximate calculation of the non equilibrium distribution:

$$
f \approx f_{0}+\frac{\boldsymbol{F}}{\hbar} \tau \cdot \nabla_{k} f_{0}-\tau \frac{\partial f_{0}}{\partial T} \boldsymbol{v} \cdot \nabla_{r} T .
$$

To find the relationships for the transport parameters we can use the expressions for the current density $\boldsymbol{J}$ and heat flux $\boldsymbol{J}_{q}$ carried by electrons:

$$
\begin{gathered}
\boldsymbol{J}(\boldsymbol{r})=\frac{1}{4 \pi^{3}} \iiint q \boldsymbol{v}(\boldsymbol{k}) f(\boldsymbol{r}, \boldsymbol{k}) d^{3} \boldsymbol{k} \\
\boldsymbol{J}_{q}(\boldsymbol{r})=\frac{1}{4 \pi^{3}} \iiint\left[E(\boldsymbol{k})-E_{f}(\boldsymbol{r})\right] \boldsymbol{v}(\boldsymbol{k}) f(\boldsymbol{r}, \boldsymbol{k}) d^{3} \boldsymbol{k}
\end{gathered}
$$

Using expression 1.7 and integrating over all possible wave vectors $k$ we obtain:

$$
\begin{gathered}
\boldsymbol{J}=q^{2} L_{0}\left(\boldsymbol{F}-\frac{1}{q} \nabla_{r} E_{f}\right)+\frac{q}{T} L_{1}\left(-\nabla_{r} T\right) \\
\boldsymbol{J}_{q}=q L_{1}\left(-\frac{1}{q} \nabla \Phi\right)+\frac{1}{T} L_{2}(\nabla T)
\end{gathered}
$$


where $\phi$ is the electrochemical potential $\left(-\nabla \phi / q=\boldsymbol{F}+\nabla E_{f} / q\right)$ and the transport coefficients $L_{n}$ are defined by the following integral:

$$
L_{n}=\frac{1}{4 \pi^{3}} \iiint \tau(\boldsymbol{k}) v(\boldsymbol{k}) v(\boldsymbol{k})\left[E(\boldsymbol{k})-E_{f}\right]^{n} f(\boldsymbol{r}, \boldsymbol{k})\left(-\frac{\partial f_{0}}{\partial E}\right) d^{3} \boldsymbol{k} .
$$

Using expressions 1.10 and 1.11, several transport properties such as the electrical conductivity, the thermal conductivity due to electrons, and the Seebeck coefficient can be determined. In the case of current flow and a temperature gradient in the $\mathrm{x}$ direction we obtain:

$$
\begin{gathered}
\sigma=\left.\frac{q J_{x}}{-\nabla \Phi}\right|_{\nabla_{x} T=0}=q^{2} L_{0} \\
S=\left.\frac{\nabla \Phi}{q \nabla_{x} T}\right|_{J_{x}=0}=\frac{1}{q T} L_{0}^{-1} L_{1} \\
\kappa_{e}=\left.\frac{J_{q_{x}}}{\nabla_{x} T}\right|_{J_{x}=0}=-\frac{1}{T} L_{1} L_{0}^{-1} L_{1}+\frac{1}{T} L_{2}
\end{gathered}
$$

The electrical conductivity and Seebeck coefficient can be rewritten in the form of integrals:

$$
\begin{gathered}
\sigma=\int \sigma(E)\left(-\frac{\partial f_{0}}{\partial E}\right) d E \\
S=\frac{k_{B}}{q} \frac{\int \sigma(E) \frac{E-E_{f}}{k_{B} T}\left(-\frac{\partial f_{0}}{\partial E}\right) d E}{\int \sigma(E)\left(-\frac{\partial f_{0}}{\partial E}\right) d E} \propto\left\langle E-E_{f}\right\rangle
\end{gathered}
$$

where we have introduced the differential conductivity:

$$
\sigma(E)=q^{2} \tau(E) \iint v_{x}^{2}\left(E, k_{y}, k_{z}\right) d k_{y} d k_{z} \approx q^{2} \tau(E) \bar{v}_{x}^{2}(E) D(E)
$$

where $D(E)$ is the density of states. The Fermi 'window' factor, which is the derivative of the Fermi distribution $\left(\partial f_{0} / \partial E\right)$ is a bell shaped function centered at $E=E_{f}$, having a width of $\sim k_{B} T$. As a consequence at a finite temperature only electrons near the Fermi surface can contribute to the conduction process, as expected by the Pauli exclusion principle ${ }^{1}$. In order to achieve a high electrical conductivity the $\sigma(E)$ within the Fermi window must be as big as possible, and at the same time it should be as asymmetric as possible with respect to the Fermi energy to achieve a high thermopower. In this case the thermopower (Seebeck coefficient) is the average energy transported by the charge carriers ${ }^{1,22}$. 


\subsubsection{Thermoelectric device}

(a)

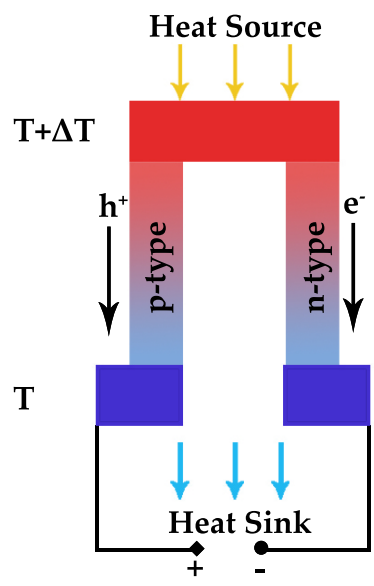

(b)

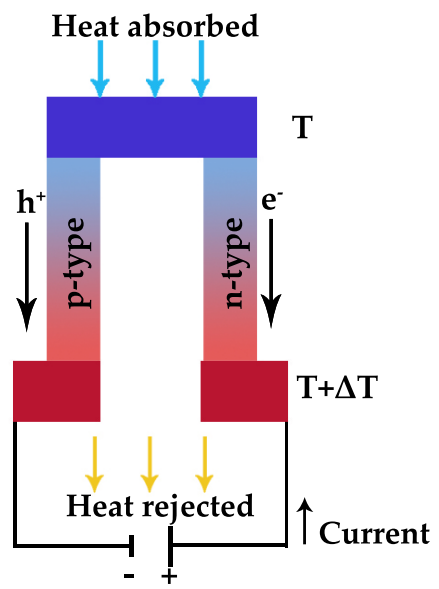

FIGURE 1.2: Basic thermoelectric device for (a) heat to electrical energy conversion and $(b)$ heat pumping.

A typical thermoelectric device for power generation is shown in Figure 1.2(a), which consists of a n-type and p-type material placed between a heat source (waste heat) and a heat sink (ambient temperature). When a temperature gradient is applied, the transfer of heat from the source to the heat sink can then occur via the diffusion of the charge carriers or through the lattice vibrations (phonons). The resulting potential difference arising from the Seebeck effect can then be collected by an external circuit. Typically thermoelectric generators are placed electrically in series and thermally in parallel. In the case of a heat pump we can use the same thermoelectric device now connected to a DC power supply as shown in Figure 1.2(b). The resulting flow of charge carriers also transports thermal energy as they flow through the circuit according to the Peltier effect. This allows the top of the thermoelectric device to be cooled while heat is rejected at the bottom.

\subsubsection{Efficiency and the figure of merit}

If we consider a basic thermocouple leg as depicted in Figure 1.2(a) connected electrically in series and thermally in parallel then the efficiency of a typical thermoelectric generator can be given by ${ }^{2,24}$ :

$$
\eta=\frac{\text { energy supplied to the load resistance }}{\text { heat energy absorbed at hot junction }} .
$$

The denominator describes the heat energy absorbed at the hot junction, which is equal to the sum of the heat conduction rate, Peltier heat absorbed at the 
hot junction due to the generated current, and the Joule heating absorbed at the hot junction ${ }^{2,24}$.

If we assume that the electrical conductivities, thermal conductivities, and Seebeck coefficients are constant over the applied temperature gradient, and that the contact resistances at the junctions are negligible compared with the sum of series resistances of the thermoelectric generator then the efficiency can be given by:

$$
\eta=\frac{I^{2} R_{L}}{K \Delta T+S T_{h} I-\frac{I^{2} R}{2}}
$$

where $R_{L}$ is the load resistance, $R$ is the resistance of the thermocouple leg, $K$ is the thermal conductance, $T_{h}$ is the temperature at the hot junction, $\Delta T$ is the temperature difference between the hot and cold junction, and $I$ is the current generated by the thermoelectric generator.

The current in a TEG is given by:

$$
I=\frac{S \Delta T}{R+R_{L}}
$$

If we define $m=\frac{R_{L}}{R}$ and substitute equation 1.21 into 1.20 we obtain:

$$
\eta=\frac{\Delta T}{T_{h}} \frac{\frac{m}{m+1}}{1+\frac{K R(m+1)}{S^{2} T_{h}}-\frac{\Delta T}{2 T_{h}(m+1)}} .
$$

By defining:

$$
Z=\frac{S^{2}}{K R}=\frac{\sigma S^{2}}{\kappa}
$$

Equation 1.22 becomes:

$$
\eta=\frac{\Delta T}{T_{h}} \frac{\frac{m}{m+1}}{1+\frac{m+1}{Z T_{h}}-\frac{\Delta T}{2 T_{h}(m+1)}} .
$$

The load resistance, $R_{L}$ is a free variable and may be chosen for any specific value of $m$. In order to find the maximum possible efficiency we must find the optimum value of $m$ that maximizes equation 1.24 . Which is given by:

$$
m_{\max }=\sqrt{1+Z T}
$$

where $T=\left(T_{h}+T_{c}\right) / 2$. Substituting into equation 1.24:

$$
\eta_{\max }=\frac{\Delta T}{T_{h}} \frac{\sqrt{1+Z T}-1}{\sqrt{1+Z T}+\frac{T_{c}}{T_{h}}}
$$


we obtain an expression for the maximum possible efficiency for a material with a given $Z$, where $Z$ is the figure of merit, which is dependant only on the properties of a material ${ }^{2,24}$. If $Z T$ approaches infinity the above reduces to the Carnot efficiency. For any TEG the maximum efficiency is dependant only on the temperature difference and the figure of merit.

\subsubsection{Figure of merit}

$Z T$ is the dimensionless thermoelectric figure of merit which is a useful yardstick for comparing the performance of thermoelectric materials.

$$
Z T=\frac{\sigma S^{2} T}{\kappa}
$$

where $\sigma$ is the electrical conductivity, $S$ is the thermopower, $\kappa$ is the thermal conductivity of the material, and $T$ is the average temperature.

Currently the most widely used materials in thermoelectric devices are based on tellurides or antimonides of transition metals or rare earth elements. The best of these commercialized materials, $\mathrm{Bi}_{2} \mathrm{Te}_{3}$ only has a $Z T \sim 1$ and generally has energy conversion efficiencies of less than $10 \%$. In order for thermoelectric materials to become more widely used, commercially viable, and competitive with traditional mechanical energy conversion systems, materials with a $Z T>3$ (energy conversion efficiencies $>15-20 \%$ ) are needed $^{1,25}$.

\subsubsection{Increasing the figure of merit}

So in order to increase the thermoelectric figure of merit we must increase both the electrical conductivity and thermopower while also decreasing thermal conductivity. In practice this can be quite difficult to achieve, as an increase in the electrical conductivity typically results in a decrease in the thermopower, which can be seen from simple models of electron transport. For metals or semiconductors the Seebeck coefficient can be expressed by ${ }^{26}$ :

$$
S=\frac{8 \pi^{2} k_{B}^{2}}{3 e h^{2}} m^{*} T\left(\frac{\pi}{3 n}\right)^{2 / 3}
$$

where $n$ is the carrier concentration and $m^{*}$ is the effective mass of the carrier. The electrical conductivity is related to the carrier concentration through the carrier mobility $\mu$ :

$$
\sigma=n e \mu \text {. }
$$




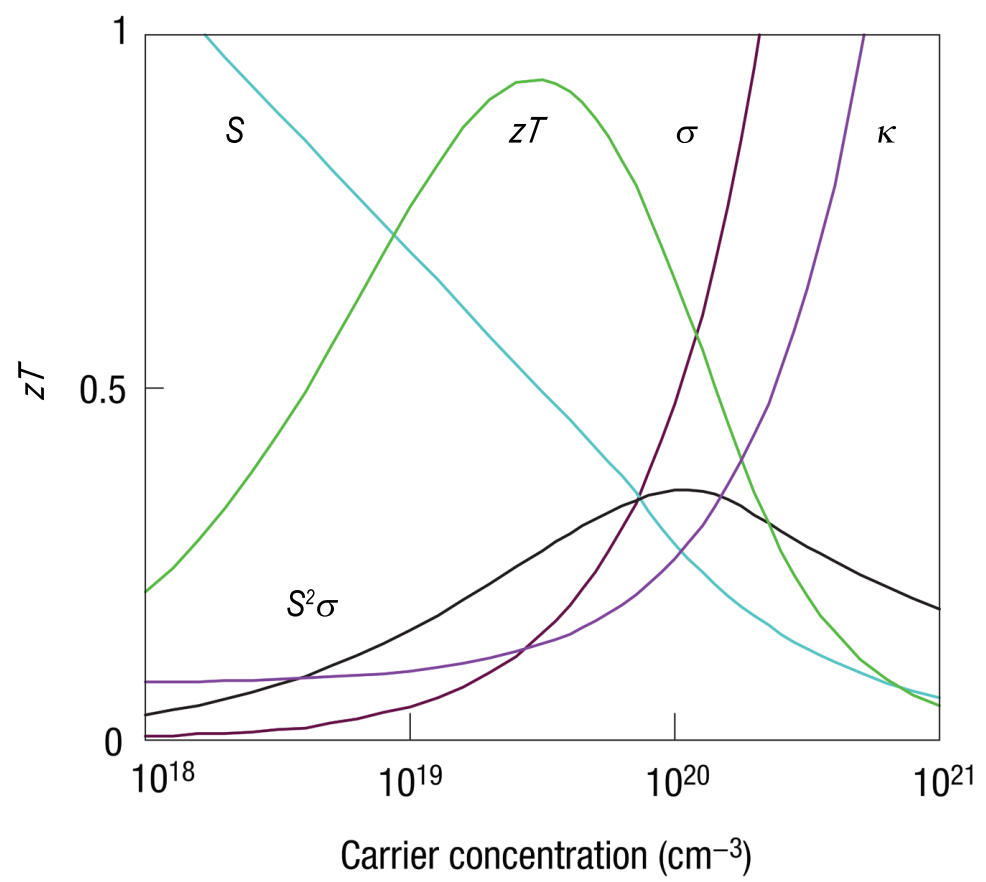

FIGURE 1.3: Optimising thermoelectric properties of $\mathrm{Bi}_{2} \mathrm{Te}_{3}$ with carrier concentration ${ }^{26}$.

Physically this can be explained by considering a simple system where there is a temperature gradient. In this case there will be an excess of high energy charge carriers on the hot side which will diffuse from hot to cold, but conversely there will also be a high number of low energy charge carriers at the cold end which results in the diffusion of the low energy carriers from cold to hot. The net drift movement of the charges will determine the magnitude of the thermopower so an increase in the charge carrier concentration leads to an increase in the rate of diffusion for both high energy and low energy charge carriers. This results in a decrease in the overall net movement of charges and thus a decrease in the thermopower while the conductivity increases. The compromise between achieving a large thermopower and high electrical conductivity in thermoelectric materials can be seen in Figure 1.3.

Furthermore the thermal conductivity has two contributions:

$$
\kappa=\kappa_{\text {lattice }}+\kappa_{\text {electronic }} \text {. }
$$

The lattice contribution is related to the transfer of heat that occurs via lattice vibrations. Atoms that vibrate more energetically can transfer energy to less energetic neighboring atoms which can result in the propagation of lattice waves (phonons) along the thermal gradient. The electronic contribution is due to the transfer of heat that occurs via the movement of high energy charge 
carriers; a high concentration of charge carriers with a high kinetic energy will diffuse along the temperature gradient to areas with a low concentration of high kinetic energy charge carriers thus transporting the thermal energy from hot to cold.

The electronic contribution to thermal conductivity is related to the electrical conductivity by the Wiedemann-Franz law which is given by

$$
\kappa_{\text {electronic }}=L_{0} T \sigma
$$

where $L_{0}=\left(\pi^{2} / 3\right)\left(k_{B} / e\right)^{2}$ is the Lorenz number. This means that an increase in the electrical conductivity will result in the increase in the electronic contribution to the thermal conductivity. This makes increasing the thermoelectric figure of merit quite difficult as all three parameters are related to charge carrier concentration as depicted in Figure 1.325,27.

\subsubsection{Advances in thermoelectric materials}

In the past decade there have been several developments in engineering materials with a $Z T>1$. Some examples are the engineering of electronic density of states in PbTe based compounds. Heremans et al. (2008) successfully implemented band structure engineering by using thallium impurity levels in lead telluride to achieve a $Z T>1.5$ at $773 \mathrm{~K}$, which is twice the $Z T$ value of p-type $\mathrm{PbTe}^{7}$. Pei et al. (2011) demonstrated that the convergence of many valleys could be achieved by tuning the doping and composition in doped $\mathrm{PbTe}_{1-\mathrm{x}} \mathrm{Se}_{\mathrm{x}}$ alloys, leading to a $Z T$ of about 1.8 at $850 \mathrm{~K}^{8}$. In this case degenerate valleys are separate pockets of Fermi surface with the same energy. Having multiple degenerate valleys has the effect of producing a large effective mass without reducing the mobility, this leads to high Seebeck coefficients without decreasing the conductivity ${ }^{8}$. Bands can be considered converged when their energy separation is small (relative to $k_{b} T$ ), effectively increasing the number of degenerate valleys. This band engineering of the valence (or conduction) bands using doping to achieve high valley degeneracy may be used as a general strategy to search for and improve bulk thermoelectric materials due to the simultaneously high Seebeck coefficient and high electrical conductivities possible.

The use of complex crystal structures have also seen a high $Z T$ being achieved. Binary skutterudites based on the mineral $\mathrm{CoAs}_{3}$ with the general formula $\mathrm{MX}_{3}(\mathrm{M}=\mathrm{Co}, \mathrm{Rh}$, or Ir; $\mathrm{X}=\mathrm{P}, \mathrm{As}$, or $\mathrm{Sb})$ have intrinsic nanoscale cages in their crystal structure due to the corner sharing $\mathrm{MX}_{6}$ octahedra. Filling of 
these nanoscale cages have been demonstrated by Shi et al. (2011) to achieve a very high $Z T$ of 1.7 at $850 \mathrm{~K}$ for $\mathrm{CoSb}_{3}$ based skutterides with multiple cofillers $\mathrm{Ba}, \mathrm{Lb}$, and $\mathrm{Yb}$. They showed that a high power factor can be realized by adjusting the total filling fraction of fillers with different charge states to reach the optimum carrier density, while at the same time the lattice thermal conductivity could be significantly reduced by using filler species of different vibrational frequencies to achieve broad-frequency phonon scattering 9 . This approach of using multiple filler with different charge states and vibrational frequencies may be a valid approach for other caged thermoelectric compounds ${ }^{9,11}$.

\subsubsection{Nanostructured thermoelectric materials}

Currently a large body of research is focused on nanostructured semiconductors where it has been shown that quantum confinement and the introduction of interfaces can lead to significant increases in the thermoelectric figure of merit. The introduction of nanostructures can introduce tall barriers (1-10 times $k_{B} T$ ) in the conduction band (n-type materials) or valence band (p-type materials), which means that only high energy carriers can be selectively transmitted through the structure as the low energy carriers are filtered out. This can lead to a substantial increase in the thermopower as the overall number of low energy carriers that can diffuse from the cold to the hot side is decreased while the diffusion of high energy charge carriers is largely unaffected by the nanostructuring ${ }^{25}$.

$$
S=\frac{\pi^{2} k^{2} T}{3 e}\left(\frac{\partial \log \sigma(E)}{\partial E}\right)_{E=E_{F}}
$$

According to the Mott relation (1.32), an increase in the thermopower may also arise from the quantum confinement in nanostructures ${ }^{28}$. Quantum confinement in zero dimensional (OD) nanostructures results in carrier confinement in all three dimensions. This gives rise to delta-like functions in the density of states (DOS), which can lead to an enhancement in the thermopower by increasing the slope of the DOS near the Fermi level. This quantum confinement effect in nanostructures has also been computationally predicted by Hicks and Dresselhaus (1993) to greatly increase the thermopower ${ }^{29,30}$. This concept of quantum confinement may have ignited intense research into the field but it has yet to be shown to play a significant role in increasing $Z T$ for nanostructured materials ${ }^{25}$. 
Although nanostructures can lead to an increase in the power factor $\left(\sigma S^{2}\right)$, the main increase in the thermoelectric figure of merit for nanostructures is due to the reduced lattice thermal conductivity that arises in these systems. In systems where we have the inclusion of nanoparticles (or atomic defects) in a matrix, we can get the scattering of mid- to long-wavelength phonons which typically propagate without significant scattering in bulk systems. This can lead to a significant reduction in the lattice thermal conductivity. It has been shown for a PbTe/PbSe nanocrystals superlattice that a lattice thermal conductivity as low as $0.33 \mathrm{~W} \mathrm{~m}^{-1} \mathrm{~K}^{-1}$ has been achieved, which is a significant decrease compared $\sim 2 \mathrm{~W} \mathrm{~m}^{-1} \mathrm{~K}^{-1}$ for PbTe bulk systems ${ }^{31}$. In nanosized polycrystalline systems we can also get the scattering of phonons at the interfaces that are introduced.

Some results of record high $Z T$ have already been achieved using nanostructured materials. Biswas et al. (2012) have recently demonstrated a $Z T$ of 2.2 at $915 \mathrm{~K}$ in p-type PbTe endotaxially nanostructured with $\mathrm{SrTe}$ at a concentration of 4 mole percent and mesostructured with powder processing and spark plasma sintering. Venkatasubramanian et al. (2001) have also demonstrated a high $Z T$ of 2.4 for p-type $\mathrm{Bi}_{2} \mathrm{Te}_{3} / \mathrm{Sb}_{2} \mathrm{Te}_{3}$ superlattice thin film based devices.

\subsubsection{Nanocomposite polycrystalline materials}

One approach to making these systems is by hot pressing or spark plasma sintering of fine powders that are produced using a wet chemistry process. These types of nanocomposite materials can have some advantages over other nanostructures. The compacting of the nanoparticles introduces extensive interfacing between the nanoparticles which can lower the thermal conductivity significantly. Furthermore we can also get an increased power factor due to electron filtering at the grain boundaries ${ }^{32}$. It should also be noted that at these interfaces the scattering of charge carriers can also occur however in most of these cases it has been demonstrated that the reduction in thermal conductivity is much stronger than the corresponding reduction in the electrical conductivity ${ }^{25}$.

Promising results have been reported using this approach. Nanosized polycrystalline BiSbTe alloy were shown to have ZT of 1.4 at $373 \mathrm{~K}$ which is $40 \%$ higher than the comparable ingot of the bulk BiSbTe alloy. The improvement in ZT for BiSbTe was mainly attributed to the strong decrease in the thermal conductivity ${ }^{4}$. 
Joshi et al. (2008) have also demonstrated a significant increase in the $Z T$ of SiGe alloys. A ZT of 0.95 was achieved for current-induced hot pressing of ball milled Si and Ge chunks with boron powder. This is $50 \%$ higher than reported p-type SiGe bulk alloys. The enhancement of $Z T$ was attributed to a large reduction in thermal conductivity due to increased phonon scattering at the grain boundaries combined with an increased power factor at higher temperatures ${ }^{6}$.

\subsection{7 $\quad \mathrm{Bi}_{2} \mathrm{Te}_{3}$ nanomaterials}

$\mathrm{Bi}_{2} \mathrm{Te}_{3}$ has already shown promise as a thermoelectric material with a $Z T \sim$ 1 for bulk $\mathrm{Bi}_{2} \mathrm{Te}_{3}$. Further increase in $Z T$ have also been reported in the literature for $\mathrm{Bi}_{2} \mathrm{Te}_{3}$ based nanostructures ${ }^{4,33}$. The improvements in the figure of merit are typically attributed to a significant reduction in the lattice thermal conductivity while having a comparable power factor to bulk $\mathrm{Bi}_{2} \mathrm{Te}_{3}$. Most of the successful attempts to increase $Z T$ in nanostructured $\mathrm{Bi}_{2} \mathrm{Te}_{3}$ have been done using the top-down approach, such as ball milling ${ }^{4,34,35}$. This approach is energy intensive, requires long processing times and results in poor control of the nanoparticle size. Bottom-up wet chemical synthesis attempts show the potential to produce nanoparticles with a much better control over the size and morphology along with being relatively cheap and requiring low energy, which allows the production of a more easily scalable method. Typical colloidal synthesis attempts of nanostructured $\mathrm{Bi}_{2} \mathrm{Te}_{3}$ show a $Z T<1$, nevertheless in most reported cases a significant reduction in the thermal conductivity has been achieved with similar electrical conductivities to bulk $\mathrm{Bi}_{2} \mathrm{Te}_{3}{ }^{33-40}$. Further optimisation of the power factor $\left(\sigma S^{2}\right)$ for nanostructured $\mathrm{Bi}_{2} \mathrm{Te}_{3}$ through doping maybe able to yield further improvements in the figure of merit for bottom-up wet chemical synthesis of nanostructured $\mathrm{Bi}_{2} \mathrm{Te}_{3}$.

\subsubsection{Alternative materials}

A wide range of materials show potential for applications as thermoelectric materials. In particular SnSe has recently been shown to have ultralow thermal conductivities in single crystals and exceptional power factors leading to record hight $Z T^{12,13,41}$. Zhao et al. (2014) reported a $Z T$ as high as 2.6 at $923 \mathrm{~K}$ in SnSe single crystals ${ }^{12}$. This is attributed to its ultrahigh power factor and exceptionally low thermal conductivity for a crystalline material. Single crystal SnSe has already proven to be an exceptional thermoelectric material ${ }^{12,13,41}$. Further improvements may be possible from nanostructuring 
of SnSe. Other materials such as organic-inorganic single crystals may also prove to be interesting novel thermoelectric materials as recent computational calculations have shown the potential to achieve a high figure of merit ${ }^{14}$.

\subsection{Organic-inorganic single crystal perovskites}

Another potential application of thermoelectric materials is in solar thermoelectric generators (STEG) ${ }^{42-44}$. The performance of a solar thermoelectric material depends on both light absorption and thermoelectric energy conversion. This requires materials with a high absorption coefficient to efficiently collect the light and turn it into heat. If the material also exhibits a large $Z T$ then they can convert the heat into electricity via the Seebeck effect ${ }^{14}$. Although a $Z T>3$ is needed for thermoelectric generators to become competitive a $Z T>1$ may already be beneficial for applications in STEG ${ }^{43}$. Recent computational calculations have shown the potential for organic inorganic halide perovskites to be used as thermoelectric materials for applications in STEG, achieving a $Z T$ between $1-2^{14}$.

Perovskite materials describe crystals that adopt the same crystal structure as $\mathrm{CaTiO}_{3}$. Where the large cation $\mathrm{A}$ is typically methylammonium (MA),

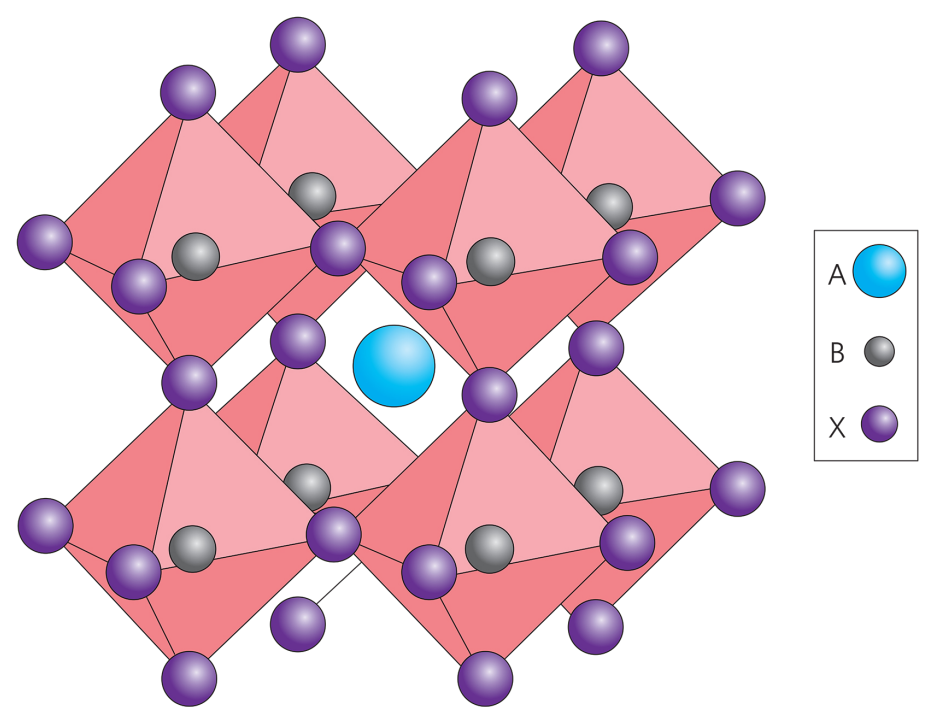

FIGURE 1.4: Perovskite crystal structure for general formula $\mathrm{ABX}_{3}{ }^{45}$.

formamidinium (FA) or $\mathrm{Cs}$, the small cation $\mathrm{B}$ is typically $\mathrm{Pb}$ or $\mathrm{Sn}$, and $\mathrm{X}$ is a halide $(\mathrm{Br}, \mathrm{Cl}$ or $\mathrm{I})$. In recent years a lot of research has been focused on 
organic-inorganic mixed halides perovskites due to their remarkable optical and electrical properties, along with being relatively cheap and solution processable. Properties such as balanced electron and hole transport ${ }^{46}$, long diffusion lengths ${ }^{47,48}$, high mobilities ${ }^{49,50}$, and large absorption coefficients in the UV-vis region ${ }^{51}$ have seen perovskites being used for photovoltaics ${ }^{15-18}$, light emitting diodes ${ }^{52,53}$, photodetectors $^{54-58}$, and lasers ${ }^{59-61}$.

Lately organic-inorganic halide single crystal perovskites have also been considered for thermoelectric applications due to their low thermal conductivity and high Seebeck coefficient ${ }^{62,63}$, along with computational predictions of high $Z T^{14}$. This has prompted the investigation into single crystal perovskites as a thermoelectric material.

\subsection{In this thesis}

In this thesis we will be investigating the thermoelectric properties of colloidal $\mathrm{Bi}_{2} \mathrm{Te}_{3}$ nanostructures. Several different synthetic approaches to produce nanostructured $\mathrm{Bi}_{2} \mathrm{Te}_{3}$ will be presented in chapter 3 along with their thermoelectric properties. We will then explore the effects of various capping ligands and how a ligand exchange treatment may be used to improve the figure of merit. The incorporation of $\mathrm{Co}, \mathrm{Ni}$ and $\mathrm{Cu}$ into nanostructured $\mathrm{Bi}_{2} \mathrm{Te}_{3}$ will then be shown along with their structural characterisation and thermoelectric properties.

In chapter 4 we will investigate the potential for using organic-inorganic single crystal perovskites as novel thermoelectric materials. A method to grow single crystal perovskites will be presented along with the development of incorporating mixed halides of $\mathrm{Br} / \mathrm{Cl}$ and mixed metals of $\mathrm{Pb} / \mathrm{Sn}$ into the single crystals. Characterisation of the optical and electrical properties for single crystals consisting of $\mathrm{MAPbBr}_{3-\mathrm{x}} \mathrm{Cl}_{\mathrm{x}}, \mathrm{FAPbBr}_{3-\mathrm{x}} \mathrm{Cl}_{\mathrm{x}}$, and $\mathrm{MAPb}_{1-\mathrm{x}} \mathrm{Sn}_{\mathrm{x}} \mathrm{Br}_{3}$ will then be discussed. 



\section{Chapter 2}

\section{Materials and Methods}

\subsection{Characterisation techniques}

\subsubsection{Transmission electron microscopy}

All the images collected in this thesis were recorded using a JEOL JEM-2100F transmission electron microscope (TEM) operated at $200 \mathrm{kV}$ and equipped with a JEOL SDD EDS detector. TEM samples were prepared by dispersing the nanoparticles in either chloroform or toluene. The dilute solutions were then drop cast onto formvar coated copper grids. Energy dispersive X-ray spectroscopy (EDS) was carried out in both STEM and TEM imaging modes. The elemental compositions are then determined using standardless quantitative analysis in the analysis station software provide by JEOL. Analysis of particle distributions are carried out using the open source software ImageJ. This is done by using the measurement tool and fitting particles using the elliptical or freehand tool. Additional use of the threshold tool may also be utilized to help identify nanoparticles more clearly.

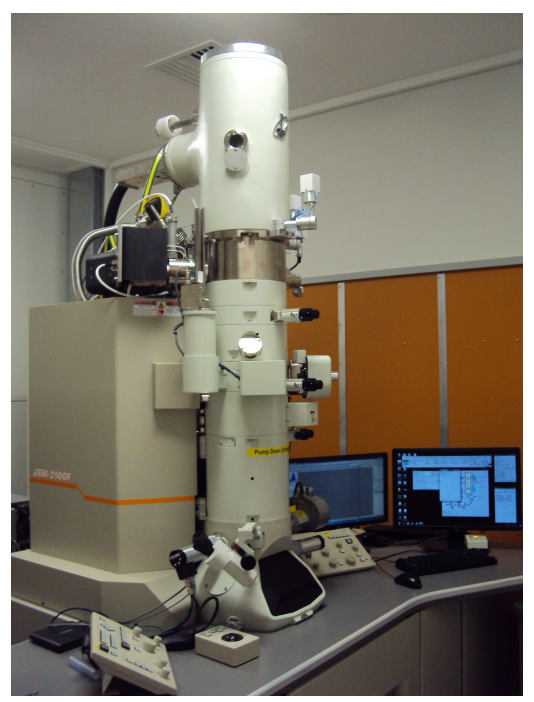

FIGURE 2.1: TEM used to characterize nanomaterials. 


\subsubsection{Scanning electron microscopy}

Scanning electron microscope (SEM) images were collected using a JEOL JSM6610 SEM with an integrated JEOL EDS detector operated in high vacuum mode with an accelerating voltage of $15 \mathrm{kV}$ and a working distance of 10 $\mathrm{mm}$. Solid samples were prepared by using double sided carbon tape on an aluminium stub. Nanoparticles were prepared by spin casting multiple thin film layers onto a glass substrate. The thin films were then placed onto an aluminium stub using double sided carbon tape. Elemental maps and EDS analysis was carried out using standardless quantitative analysis using the analysis software provided by JEOL. In general elemental compositions are determined from the average of several scans at different positions. It should be noted that due to the higher penetration depth, area analysed, and counts present the EDS analysis in the SEM is a better representation of the overall macroscopic composition then the EDS analysis using the TEM.

\subsubsection{Powder X-ray diffraction}

Powder X-ray diffraction (XRD) spectra were collected using a PANalytical $X^{\prime}$ Pert Pro diffractometer using a $\mathrm{Cu}-\mathrm{K} \alpha$ radiation source and PIXcel detector. Powder XRD samples were prepared by grinding a sample into a fine powder, the powder is then placed onto a Si substrate and gently flattened with a spatula. Additionally samples were prepared by making a super concentrated solution in chloroform and drop casting it onto a glass substrate. Phase identification was achieved using Highscore+ software provided by PANalytical.

\subsubsection{Fourier transform infrared spectroscopy}

Absorption spectra are obtained using a Bruker Tensor 27 with an attached attenuated total reflection (ATR) stage. Spectra are collected by directly drop casting samples from a volatile solvent such as chloroform onto the ATR crystal.

\subsubsection{Optical characterisation}

Steady state absorption was carried out on a Cary 50 UV-vis spectrophotometer. Solid samples are placed in a quartz $10 \mathrm{~mm}$ or $1 \mathrm{~mm}$ cuvette (depending on the sample size). The samples are then placed into the beam path using the white light alignment mode. 
Photoluminescence (PL) emission and decay were carried out on a Horiba Jobin Yvon Fluorolog/Fluorohub setup. A xeon lamp was used as the source for PL emission and a $265 \mathrm{~nm}$ NanoLed was used as the source for time correlated single photon counting (TCSPC). PL decay data was analysed using the DAS6 software provided by Horiba to fit various exponential models. The prompt (instrument response) spectra were collected using $0.004 \mathrm{wt} \%$ Ludox silica in distilled water.

\subsection{Thermoelectric measurements}

Transport properties of thermoelectric materials are measured using the Physical Property Measurement System(PPMS) - Thermal transport option from Quantum Design at the Robinson Research Institute. The PPMS can measure the thermal conductivity, resistivity, and Seebeck coefficient simultaneously.

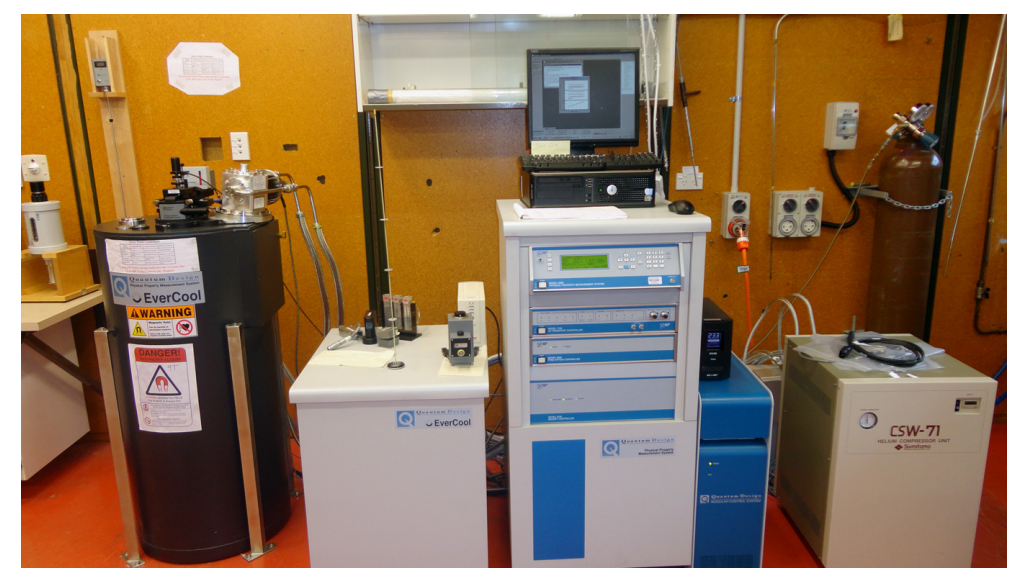

FIGURE 2.2: Physical Property Measurement System used to measure transport properties.

\subsubsection{Thermal conductivity}

The PPMS measures the thermal conductivity by applying heat from the heater shoe in order to create a user-specified temperature differential between the two thermometer shoes. The system then dynamically models the thermal response of the sample to the low-frequency, square wave heat pulse, which helps expedite data acquisition. The thermal conductivity can then be expressed as:

$$
\kappa=\frac{L}{A} \frac{I^{2} R_{\text {heater }}-\text { losses }}{\Delta T}
$$


where the "losses" are due to radiation and conduction from the sample and shoe assemblies. The thermal conductivity can then be directly calculated from the applied heater power, resulting $\Delta T$, and sample geometry ${ }^{64}$.

\subsubsection{Seebeck coefficient}

The PPMS determines the Seebeck coefficient by creating a specified temperature drop between the two thermometer shoes, just as it does to measure thermal conductivity. However, for the Seebeck coefficient the voltage drop created between the thermometer and shoes is also monitored. The expression for the Seebeck coefficient is given by:

$$
S=\frac{\Delta V}{\Delta T}-S_{\text {manganin }}
$$

where $S_{\text {manganin }}$ accounts for the thermopower of the manganin leads of the shoe assemblies ${ }^{64}$.

\subsubsection{Electrical resistivity}

The PPMS measures the electrical resistivity by using a precision digital signal processor current source and phase sensitive voltage detection. The resistivity is then given by:

$$
\rho=\frac{A}{l} R_{\text {sample }}
$$

where $A$ is the cross sectional area and $l$ is the thickness of the sample ${ }^{64}$.

\subsubsection{Software modelling}

The uncertainty for the thermal conductivity is determined by accounting for the residuals of the curve fit, the error in the heater current due to the digitalanalog converter, the error in estimating the sample radiation term where $20 \%$ combined error in the estimation of sample surface area and emissivity is assumed, and the error in the thermal conductance leak from the shoe assemblies. Further information about error analysis determined using the software provided by Quantum Design can be found in PPMS user manual ${ }^{64}$. 


\subsubsection{Sample preparation}

\subsubsection{Nanocomposite pellets}

Typically the nanomaterials prepared for thermoelectric measurements are dried under vacuum in volatile solvent such as chloroform or acetonitrile. The resulting powder is collected and further ground into a fine powder using a pestle and mortar if necessary. The powder is then collected and made into a pellet using cold isostatic pressing (CIP). Either a $5 \mathrm{~mm}$ or $6 \mathrm{~mm}$ diameter stainless steel die is used to press the pellets. The dies are typically pressed using a hydraulic press held at $\sim 0.5$ tonne $(250 \mathrm{MPa}$ for $5 \mathrm{~mm}$ or $170 \mathrm{MPa}$ for $6 \mathrm{~mm}$ die) for 5 minutes.

\subsubsection{Mounting samples for PPMS}

The nanocomposite pellets can then be mounted onto two clean copper electrodes using Epotek H20E silver epoxy according to the schematic in Figure 2.3(a). Once mixed the epoxy can be applied in a very thin layer on to the surface of the pellet. Care must be taken not to create a short when applying the silver epoxy. Once the surface of the pellet is covered with silver epoxy, it can then be placed onto a cleaned bottom copper electrode. Another layer of epoxy can then be placed on top of the pellet and a copper electrode can be placed onto it. The mounted sample is then cured overnight in an oven at 80 ${ }^{\circ} \mathrm{C}$ under argon.

(a)

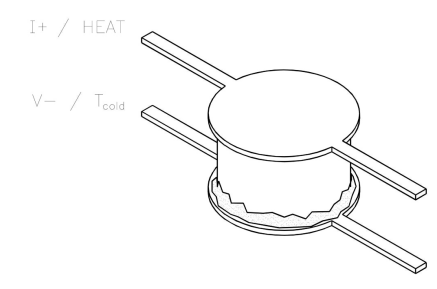

(b)

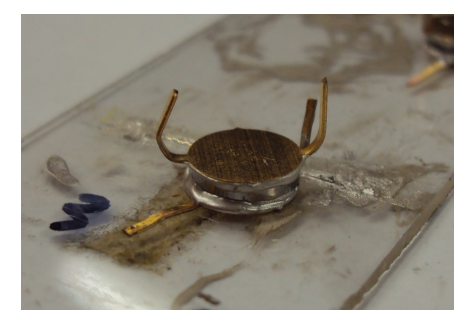

FIGURE 2.3: (a) Schematic of two-probe lead configuration using disk-shaped copper leads ${ }^{64}$. (b) $\mathrm{Bi}_{2} \mathrm{Te}_{3}$ pellet mounted using two-probe lead configuration for thermoelectric measurements. 



\section{Chapter 3}

\section{Synthesis and characterisation of nanostructured $\mathrm{Bi}_{2} \mathrm{Te}_{3}$}

Bulk $\mathrm{Bi}_{2} \mathrm{Te}_{3}$ and its alloys have long been known as one of the best thermoelectric materials with a figure merit close to unity ${ }^{1,2}$. However $Z T>3$ are needed in order to produce thermoelectric devices with efficiencies similar to those of conventional heat generators, making them commercially viable and competitive with traditional mechanical devices. The use of nanostructures to increase the figure of merit have seen further improvements to $\mathrm{Bi}_{2} \mathrm{Te}_{3}$ based materials. This is generally attributed to having comparable power factors $\left(\sigma S^{2}\right)$ with significantly reduced lattice thermal conductivities ${ }^{33-40}$. Typically these nanostructured $\mathrm{Bi}_{2} \mathrm{Te}_{3}$ materials have been synthesized using a top-down approach in which a bulk material is broken down into smaller nano sized particles ${ }^{4,34,35}$. Poudel et al. (2008) used ball milling of bulk $\mathrm{Bi}_{2} \mathrm{Te}_{3}$ to produce nanostructured $\mathrm{Bi}_{2} \mathrm{Te}_{3}$ with a $Z T=1.4$ at $100 \mathrm{~K}^{4}$. These top-down approaches are typically energy intensive, require long processing times, and lack a fine control over the size of the nanocrystalline domains. The bottom-up approach consists of building nanomaterials from smaller building blocks such as atoms. Bottomup approaches such as colloidal synthesis enables more control over the size and morphology whilst requiring low energy use enabling scalable methods and low cost alternatives compared to top-down approaches or vacuum based bottom-up approaches. Colloidal synthesis attempts of nanostructured $\mathrm{Bi}_{2} \mathrm{Te}_{3}$ have already seen impressive $Z T^{33,36-40}$. Mehta et al. (2012) produced a bottom-up assembly of $\mathrm{Bi}_{2} \mathrm{Te}_{3}$ and its alloys achieving a $Z T$ as high as 1.1. In most cases bottom-up wet chemical synthesis attempts show a $Z T<1$ but the nanostructuring has lead to a significant reduction in the thermal conductivity and comparable electrical conductivities ${ }^{33,38-40}$. This shows the potential of using nanostructured $\mathrm{Bi}_{2} \mathrm{Te}_{3}$, where further optimizations of the electrical conductivity and thermopower through dopants could lead to even higher figure of merits. Most reported colloidal synthesis routes of nanostructured 
$\mathrm{Bi}_{2} \mathrm{Te}_{3}$ result in a Bi rich phase being present and a morphology consisting of nanoplates of varying sizes ${ }^{33,36-40}$. This makes achieving stoichiometric monodisperse nanoparticles of $\mathrm{Bi}_{2} \mathrm{Te}_{3}$ an interesting challenge.

In this chapter we present results on several initial synthesis attempts to produce nanostructured $\mathrm{Bi}_{2} \mathrm{Te}_{3}$ and the improvements needed to increase the figure of merit. The effects of various ligands on the morphology and thermoelectric properties were then explored and a ligand exchange treatment was investigated to improve the figure of merit. A method to produce more monodisperse nanoparticles was found and the effect of $\mathrm{Cu}, \mathrm{Ni}$ and $\mathrm{Co}$ dopants on the morphology and thermoelectric properties was investigated.

\subsection{Experimental}

\subsubsection{Materials}

Tellurium powder (-200 mesh, 99.8\%), trioctylphosphine (TOP, 97\%), bismuth(III) chloride $\left(\mathrm{BiCl}_{3}, \geq 98 \%\right)$, oleic acid (OA, $\left.\geq 99 \%\right)$, technical grade oleic acid (OA, $90 \%$ ), oleylamine (OLA, $\geq 98 \%$ ), technical grade oleylamine (OLA, 70\%), thioglycolic acid (TGA, $>98 \%$ ), 1-dodecanethiol (DDT, $\geq 98 \%$ ), hydrazine (1.0 $\mathrm{M}$ in tetrahydrofuran), 1,5-pentanediol (purum, $\geq 97 \%$ ), bismuth(III) acetate (Bi(Ac) $)_{3} \geq 99.99 \%$ ), selenium powder (-100 mesh, $\geq 99.5 \%$ ), selenium pellets ( $<5 \mathrm{~mm}, \geq 99.99 \%$ ), tin(II) chloride $\left(\mathrm{SnCl}_{2}, \geq 98 \%\right.$ ), copper(II) acetylacetonate ( $\mathrm{CuAcAc}, 97 \%)$, 1-octadecene (ODE, 90\%), and silver conductive paste ( $\alpha$-terpineol dispersion) were purchased from Sigma Aldrich. Acetone ( $\geq 99.5 \%$ ), ethanol $(\geq 99.5 \%$ ), and isopropanol (IPA, $\geq 99.5 \%$ ) were purchased from Fisher Scientific. Acetonitrile (ACN, HPLC grade) and chloroform $\left(\mathrm{CHCl}_{3}, 99 \%\right)$ were purchased from PanReac. Octadecylphosphonic acid (ODPA) was purchased from AK Scientific. Toluene (AR grade) was purchased from Pure Science. Ammonium sulphide (10\% solution) was purchased from Unilab. Formamide (AnalaR grade) was purchased from BDH. Nickel(II) acetate tetrahydrate was purchased from Merck. Cobalt(II) acetate tetrahydrate was purchased from AJAX. All chemicals were used without further purification. 


\subsection{Initial $\mathrm{Bi}_{2} \mathrm{Te}_{3}$ synthesis attempts}

\subsubsection{Synthesis via bismuth(III) oleate}

Initial attempts to synthesize $\mathrm{Bi}_{2} \mathrm{Te}_{3}$ nanostructures were done using a modified version of the method developed by Stavila et al. $(2013)^{38}$. In this reaction $\mathrm{Bi}_{2} \mathrm{Te}_{3}$ is synthesized via a bismuth(III) oleate precursor. The following reactions are carried out using standard Schlenk line techniques under a vacuum/nitrogen.

Synthesis of bismuth(III) oleate precursor solution. $\mathrm{BiCl}_{3}(0.6356 \mathrm{~g}, 2$ mmol) was added to a round bottom flask and evacuated on a Schlenk line for 30 minutes. $5 \mathrm{~mL}$ of oleic acid was then added and the solution was degassed at $100{ }^{\circ} \mathrm{C}$ for 30 minutes, upon which the white powder dissolves to form a black solution. The flask was then purged with nitrogen for 30 minutes at 100 ${ }^{\circ} \mathrm{C}$ and then adjusted to the desired temperature.

Synthesis of TOP-Te solution. In a glove box filled with nitrogen Te powder $(0.3828 \mathrm{~g}, 3 \mathrm{mmol})$ was added to $6 \mathrm{~mL}$ of TOP. The solution was then transferred onto a nitrogen filled Schlenk line and heated to $180{ }^{\circ} \mathrm{C}$ with vigorous stirring. After $\sim 1$ hour all the Te had dissolved to form a yellow solution. The temperature was then adjusted and used immediately to synthesize $\mathrm{Bi}_{2} \mathrm{Te}_{3}$.

Synthesis of $\mathrm{Bi}_{2} \mathrm{Te}_{3}$ nanoparticles. In a typical reaction the $\mathrm{Bi}\left(\right.$ oleate) ${ }_{3}$ and TOP-Te solution were adjusted to the set reaction temperature $\left(50^{\circ} \mathrm{C}\right.$ or $\left.100{ }^{\circ} \mathrm{C}\right)$. The TOP-Te solution was than injected into the $\mathrm{Bi}$ (oleate) $)_{3}$ precursor and the solution was then held at the reaction temperature $\left(50{ }^{\circ} \mathrm{C}\right.$ or $\left.100{ }^{\circ} \mathrm{C}\right)$ overnight ( $\sim 16$ hours). The reaction was then cooled to room temperature and at $\sim 50$ ${ }^{\circ} \mathrm{C} 5 \mathrm{~mL}$ of toluene was added to prevent the formation of a gel. An equal volume of ethanol was then added to precipitate the $\mathrm{Bi}_{2} \mathrm{Te}_{3}$ nanoparticles, the nanoparticles were then washed several times by adding toluene and ethanol followed by centrifugation for 8 minutes at 8000 RPM. The nanoparticles were then dried under vacuum and the fine powder was collected and stored in a vacuum desiccator.

\subsubsection{Characterisation and thermoelectric properties}

Powder XRD spectra were obtained for the products synthesised at $50{ }^{\circ} \mathrm{C}$ and $100{ }^{\circ} \mathrm{C}$ and are shown in Figure 3.1(a). The XRD spectra indicate that a crystalline product is formed, which can be indexed to rhombohedral $\mathrm{Bi}_{2} \mathrm{Te}_{3}$ (ICDD: 04-004-8858). The FWHM of the XRD peaks indicates an increase in the 
(a)

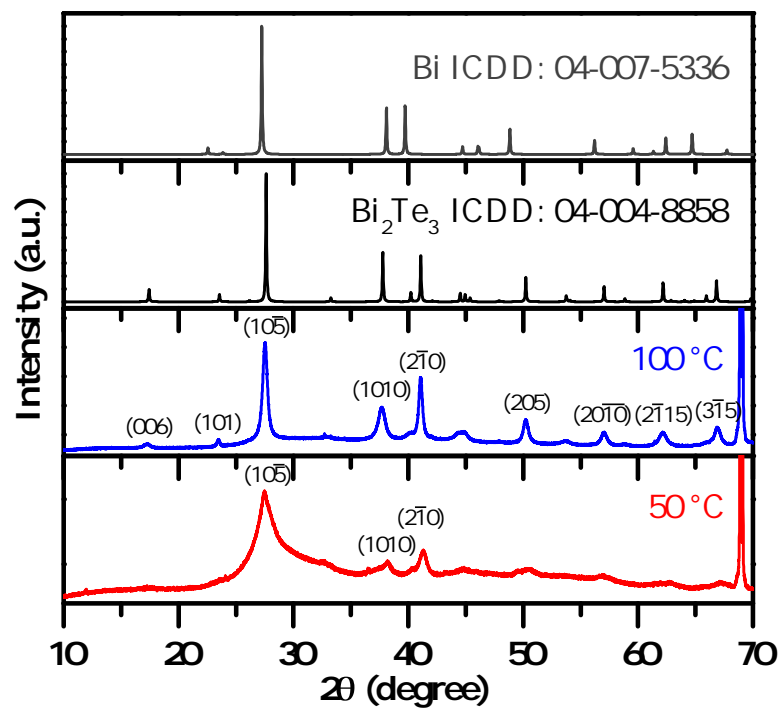

(b)

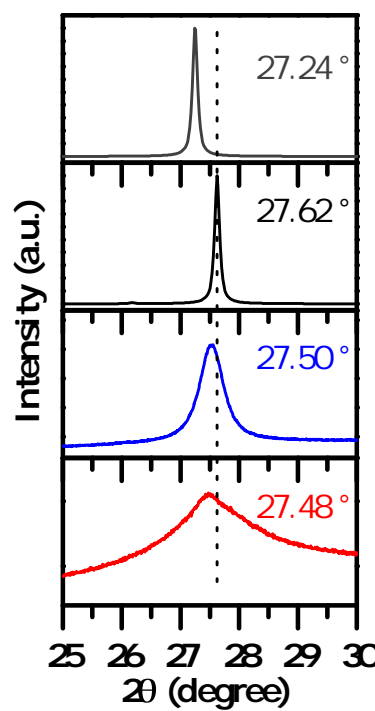

Figure 3.1: (a) Powder XRD spectra of $\mathrm{Bi}_{2} \mathrm{Te}_{3}$ synthesized at 50 ${ }^{\circ} \mathrm{C}$ and $100{ }^{\circ} \mathrm{C}$ from bismuth(III) oleate and TOP-Te. Note the peak at $69^{\circ}$ is due to the Si substrate. (b) XRD spectra showing a small shift for the $(10 \overline{5})$ peak.

(a)

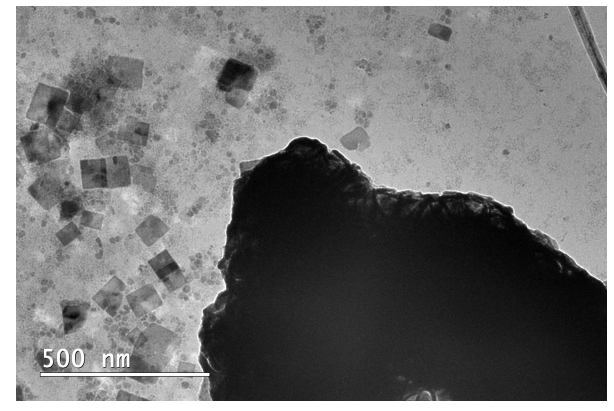

(c)

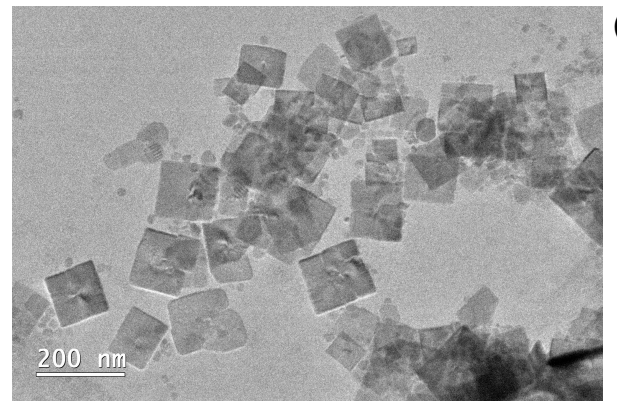

(b)

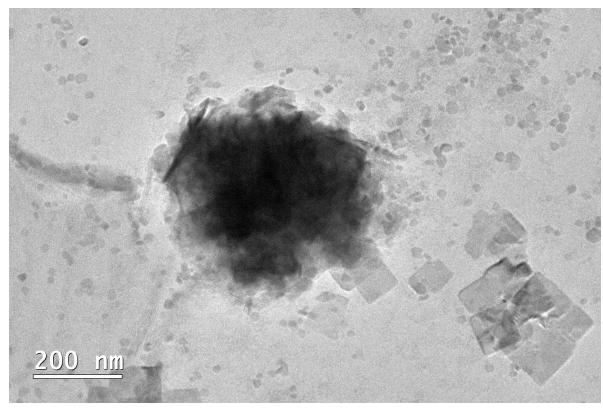

(d)

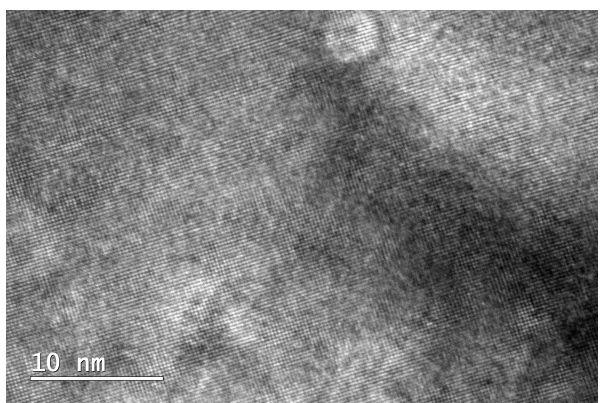

FIGURE 3.2: TEM micrographs of $\mathrm{Bi}_{2} \mathrm{Te}_{3}$ synthesised at $50{ }^{\circ} \mathrm{C}$ via bismuth(III) oleate and TOP-Te.

crystallite size with increased temperature. From the fits of the XRD pattern and using Scherrer's equation, the relative crystallite sizes were estimated to be 5 and $16 \mathrm{~nm}$ for $50{ }^{\circ} \mathrm{C}$ and $100{ }^{\circ} \mathrm{C}$ products respectively.

TEM micrographs and elemental composition of $\mathrm{Bi}_{2} \mathrm{Te}_{3}$ synthesised at $50{ }^{\circ} \mathrm{C}$ are shown in Figure 3.2 and Table 3.1. The TEM micrographs show two different nanostructures present. One appears to have agglomerated, 
where the agglomerates are typically $>200 \mathrm{~nm}$ in size and may consist of thin nanoplates. The agglomeration is most likely due to the poor colloidal stability of $\mathrm{Bi}_{2} \mathrm{Te}_{3}$ nanoparticles, which is typically $<10$ mins. The other nanostructure appears to be fairly dispersed square-like thin nanoplates. EDS indicates that the dispersed square nanoplates consist of only bismuth whereas the agglomerated particles consist of both $\mathrm{Bi}$ and Te. The elemental composition also suggests an excess of bismuth relative to stoichiometric $\mathrm{Bi}_{2} \mathrm{Te}_{3}$.

TABLE 3.1: Composition determined by TEM/EDS for $\mathrm{Bi}_{2} \mathrm{Te}_{3}$ synthesized at $50{ }^{\circ} \mathrm{C}$ via bismuth(III) oleate and TOP-Te.

\begin{tabular}{ccc}
\hline \multirow{2}{*}{ Reference image } & \multicolumn{2}{c}{ Normalized relative elemental composition } \\
\cline { 2 - 3 } & $\mathbf{B i}$ & $\mathbf{T e}$ \\
\hline 1d-x10k & 2.00 & 1.56 \\
1e-x15k & 2.00 & 1.47 \\
$2 \mathrm{~b}-x 20 \mathrm{k}$ & 2.00 & 0.92 \\
\cline { 2 - 3 } & $\sigma$ for Te composition & 0.28 \\
\hline Average & $\mathbf{2 . 0 0}$ & $\mathbf{1 . 3 2}$ \\
\hline
\end{tabular}

TEM micrographs and EDS elemental composition for $\mathrm{Bi}_{2} \mathrm{Te}_{3}$ synthesised at $100{ }^{\circ} \mathrm{C}$ are shown in Figure 3.3 and Table 3.2. The TEM micrographs shows aggolomerated materials consisting of nanoplates. The increased temperature seems to have transitioned the square-like bismuth plates into larger intergrown plates of $\mathrm{Bi}_{2} \mathrm{Te}_{3}$, which seem to be more thermodynamically stable. This can be seen from the elemental composition shown in Table 3.2 in which the Te has increased significantly relative to the sample synthesised at $50{ }^{\circ} \mathrm{C}$. However the EDS analysis still indicates a Bi rich material is formed, which is consistent with the peak shift observed in Figure 3.1(b). The morphology is similar to previous $\mathrm{Bi}_{2} \mathrm{Te}_{3}$ materials synthesized from solution ${ }^{38,39,65,66}$. The $\mathrm{Bi}$ rich phase may be due to the layered rhombohedral crystal structure of $\mathrm{Bi}_{2} \mathrm{Te}_{3}$, in which bismuth can be easily incorporated between the hexagonal planes ${ }^{67}$. This has previously been shown to lead to $\mathrm{Bi}$ rich phases of $\left(\mathrm{Bi}_{2}\right)_{\mathrm{x}}\left(\mathrm{Bi}_{2} \mathrm{Te}_{3}\right)_{\mathrm{y}}{ }^{67}$. If we assume all the $\mathrm{Te}$ forms $\mathrm{Bi}_{2} \mathrm{Te}_{3}$ and we have a similar $\mathrm{Bi}$ rich phase $\left(\mathrm{Bi}_{2}\right)_{\mathrm{x}}\left(\mathrm{Bi}_{2} \mathrm{Te}_{3}\right)_{\mathrm{y}}$. Then we have a 1:1 ratio of $\mathrm{Bi} / \mathrm{Bi}_{2} \mathrm{Te}_{3}$ phases present. It is also possible that we may have Bi nanoplates agglomerated together with $\mathrm{Bi}_{2} \mathrm{Te}_{3}$ nanoplates.

Nanocomposite pellets were then made using the dried nanoparticles and a $6 \mathrm{~mm}$ die. The resulting pellets were then annealed at $200{ }^{\circ} \mathrm{C}$ in a vacuum oven. Before annealing the point to point resistance across the pellet was 350 $\pm 50 \mathrm{k} \Omega$ and $25 \pm 5 \Omega$ for the $50{ }^{\circ} \mathrm{C}$ and $100{ }^{\circ} \mathrm{C}$ sample respectively. After 
(a)

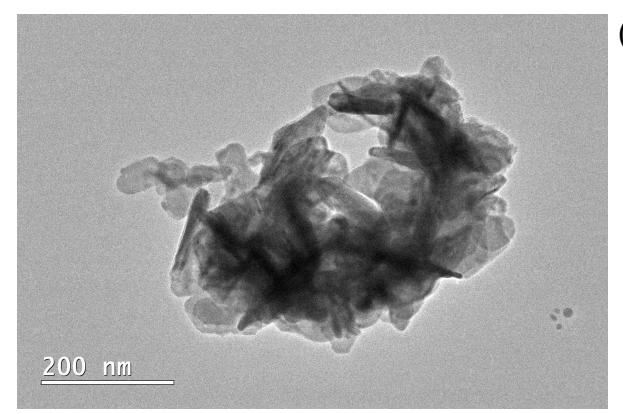

(c)

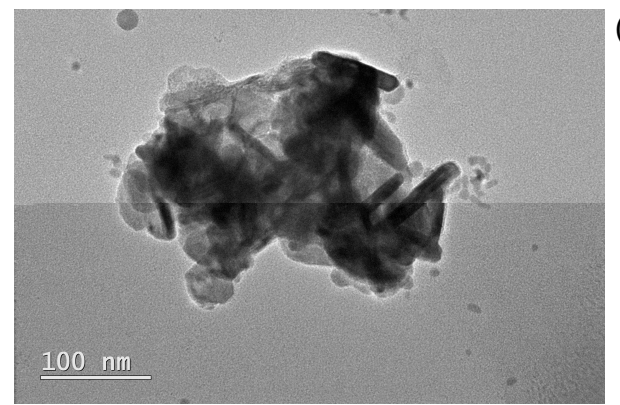

(b)

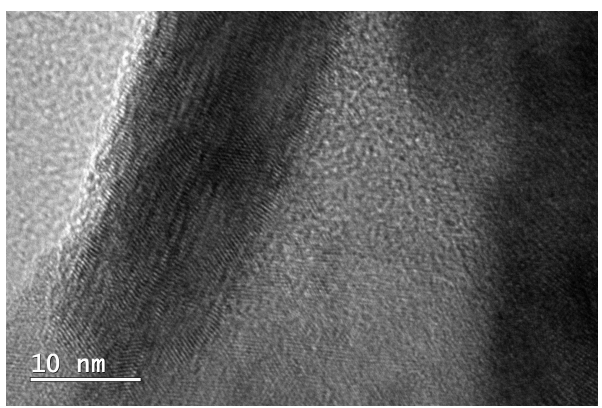

(d)

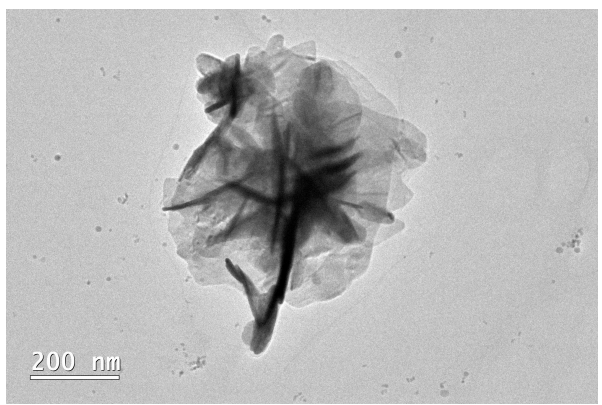

FIGURE 3.3: TEM micrographs of $\mathrm{Bi}_{2} \mathrm{Te}_{3}$ synthesised at $100{ }^{\circ} \mathrm{C}$ via bismuth(III) oleate and TOP-Te.

TABLE 3.2: Composition determined by TEM/EDS for $\mathrm{Bi}_{2} \mathrm{Te}_{3}$ synthesized at $100{ }^{\circ} \mathrm{C}$ via bismuth(III) oleate and TOP-Te.

\begin{tabular}{ccc}
\hline \multirow{2}{*}{ Reference image } & \multicolumn{2}{c}{ Normalized relative elemental composition } \\
\cline { 2 - 3 } & $\mathbf{B i}$ & $\mathbf{T e}$ \\
\hline 1a-x30k & 2.00 & 2.12 \\
1b-x50k & 2.00 & 1.95 \\
1c-x10k & 2.00 & 2.08 \\
1d-x20k & 2.00 & 1.92 \\
1d-x25k & 2.00 & 2.10 \\
\cline { 2 - 3 } & $\sigma$ for Te composition & 0.08 \\
\hline Average & $\mathbf{2 . 0 0}$ & $\mathbf{2 . 0 3}$ \\
\hline
\end{tabular}

annealing the resistance of the pellets was $>20 \mathrm{M} \Omega$ and $280 \pm 20 \Omega$ respectively. The increase in the resistance is most likely due to oxygen still being present in our vacuum oven leading to oxidation of the pellet.

The thermoelectric properties of the annealed pellets were then measured and are shown in Figure 3.4. Note that due to the high resistance of the sample synthesized at $50{ }^{\circ} \mathrm{C}$ the electrical conductivity could not be measured using the PPMS. We can see that with increased synthesis temperature the conductivity of the nanocomposite pellet increases. This may be due to increased crystallinity of the higher temperature sample as indicated by the reduced FWHM in the XRD spectra or perhaps it may be due to the additional Bi 
(a)

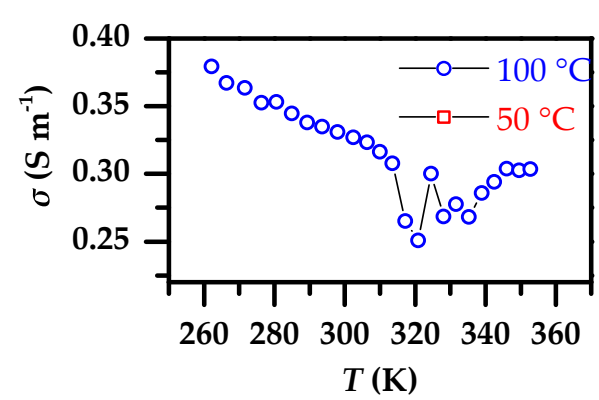

(c)

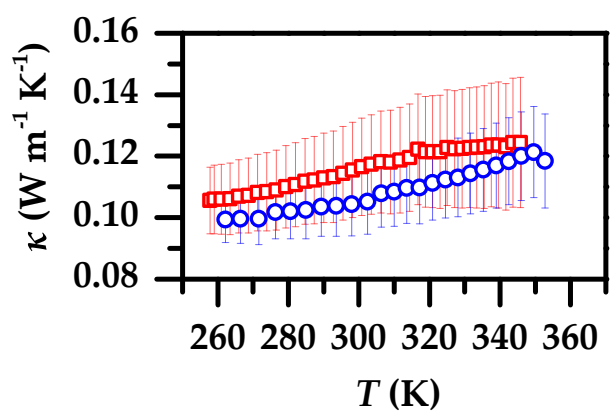

(b)

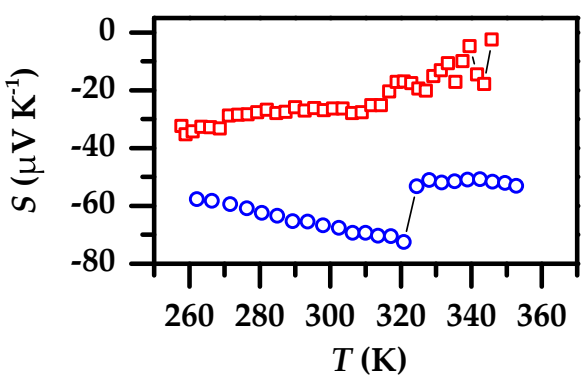

(d)

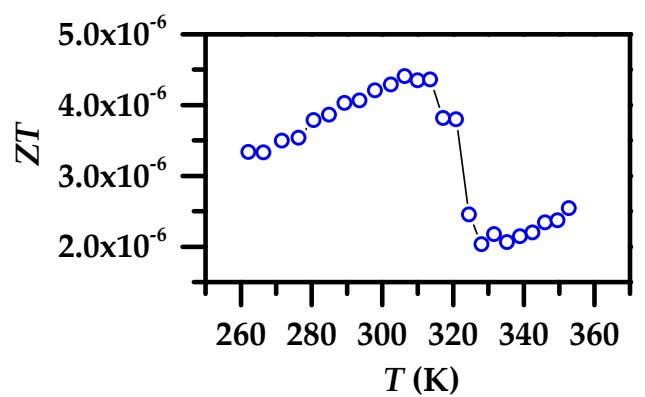

FIGURE 3.4: (a) Electrical conductivity, (b) thermopower, (c) thermal conductivity, and (d) figure of merit of $\mathrm{Bi}_{2} \mathrm{Te}_{3}$ synthesized at $50{ }^{\circ} \mathrm{C}$ and $100{ }^{\circ} \mathrm{C}$ via bismuth(III) oleate and TOP-Te.

nanoplates present for the sample synthesized at $50{ }^{\circ} \mathrm{C}$. The conductivity at room temperature is $\sim 0.33 \mathrm{~S} \mathrm{~m}^{-1}$ for the sample synthesized at $50{ }^{\circ} \mathrm{C}$ which is significantly lower than the $90000 \mathrm{~S} \mathrm{~m}^{-1}$ of bulk $\mathrm{Bi}_{2} \mathrm{Te}_{3}$ or other nanostructured $\mathrm{Bi}_{2} \mathrm{Te}_{3}$ samples ${ }^{2,33,38,40,67}$. The sign of thermopower indicates that we have made $n$-type $\mathrm{Bi}_{2} \mathrm{Te}_{3}$, where an increase in the reaction temperature appears to result in a higher thermopower. At room temperature the absolute thermopower of $\mathrm{Bi}_{2} \mathrm{Te}_{3}$ synthesized at $50{ }^{\circ} \mathrm{C}$ and $100{ }^{\circ} \mathrm{C}$ are $\sim 25 \mu \mathrm{V} \mathrm{K}^{-1}$ and $\sim 65 \mu \mathrm{V} \mathrm{K}{ }^{-1}$ respectively, which is significantly lower than the bulk $\mathrm{n}-\mathrm{Bi}_{2} \mathrm{Te}_{3}$ thermopower of $-180 \mu \mathrm{VK}^{-1} \quad 2$. The difference in the thermopower between the samples synthesized at $50{ }^{\circ} \mathrm{C}$ and $100{ }^{\circ} \mathrm{C}$ is most likely due to the increased amount of Bi present for the $50{ }^{\circ} \mathrm{C}$ samples, as bulk Bi has a significantly lower thermopower than bulk $\mathrm{Bi}_{2} \mathrm{Te}_{3}{ }^{67}$. Additionally the temperature dependent slope of the absolute thermopower is negative for the sample at $50{ }^{\circ} \mathrm{C}$ and positive for the sample at $100{ }^{\circ} \mathrm{C}$. This difference is most likely due to the compositional differences. Both pure $\mathrm{Bi}_{2} \mathrm{Te}_{3}$ and $\mathrm{Bi}$ are n-type semiconductors. However it has been shown by Bos et al. (2007) that if we have Bi incorporated between the hexagonal planes of $\mathrm{Bi}_{2} \mathrm{Te}_{3}$ so that we form a $\left(\mathrm{Bi}_{2}\right)_{\mathrm{x}}\left(\mathrm{Bi}_{2} \mathrm{Te}_{3}\right)_{\mathrm{y}}$ phase, then a high Bi content ( $\mathrm{x}: \mathrm{y}=2: 1)$ will form a p-type semiconductor (positive thermopower), while a low Bi content ( $x: y=1: 2)$ will form a n-type semiconductor (negative thermopower) ${ }^{67}$. If we have both a pure Bi phase and a 
$\left(\mathrm{Bi}_{2}\right)_{\mathrm{x}}\left(\mathrm{Bi}_{2} \mathrm{Te}_{3}\right)_{\mathrm{y}}$ phase present in the sample at $50{ }^{\circ} \mathrm{C}$ then the different contributions from each phase may explain the different temperature dependent slopes observed in Figure 3.4(b). The thermal conductivity of our nanostructured $\mathrm{Bi}_{2} \mathrm{Te}_{3}$ samples is significantly lower than the bulk $\mathrm{Bi}_{2} \mathrm{Te}_{3}$. There appears to be no significant difference (within experimental uncertainties) between the sample at $50{ }^{\circ} \mathrm{C}$ and $100{ }^{\circ} \mathrm{C}$. At room temperature the thermal conductivity of our nanostructured $\mathrm{Bi}_{2} \mathrm{Te}_{3}$ is $\sim 0.11 \mathrm{~W} \mathrm{~m}^{-1} \mathrm{~K}^{-1}$ which is significantly lower then the bulk and nanostructured $\mathrm{Bi}_{2} \mathrm{Te}_{3}$ which has a optimal thermal conductivity of $\sim 0.8 \mathrm{~W} \mathrm{~m}^{-1} \mathrm{~K}^{-1} \quad 2,33,40$. Even with the significant reduction in the thermal conductivity the figure of merit of our nanostructured $\mathrm{Bi}_{2} \mathrm{Te}_{3}$ sample is relatively low due to the poor electrical conductivity of our sample. Our next goal will be to try increase the electrical conductivity of our nanoparticles by investigating alternative surface capping ligands.

\subsubsection{Synthesis with different capping ligands}

The effects of various ligands on the morphology of $\mathrm{Bi}_{2} \mathrm{Te}_{3}$ synthesised via bismuth(III) oleate was carried out using different capping ligands such as oleylamine (OLA) and 1-dodecanethiol (DDT).

$\mathrm{Bi}_{2} \mathrm{Te}_{3}$ synthesised via bismuth oleate capped with OLA or DDT. Modifications to the reaction in subsection 3.2.1 was carried out to investigate the effect of different ligands. In order to investigate the effects of the ligands, 20 $\mathrm{mL}$ of oleylamine (or 1-dodecanethiol) was added to the Bi(oleate) ${ }_{3}$ precursor after the degassing step. The rest of the reaction is carried out in the same manor except the reaction temperature for $\mathrm{Bi}_{2} \mathrm{Te}_{3}$ is now set to $120{ }^{\circ} \mathrm{C}$.

The effect of thioglycolic acid was also investigated using a modified version of the method produced by Mehta et al. (2012) ${ }^{33}$. In this method nanoparticles are prepared from a thioligated bismuth complex and TOP-Te. The use of thioglycolic acid as a capping ligand can lead to sulphur doped pnictogen chalcogenides with enhanced conductivity ${ }^{33}$.

Synthesis of thioligated bismuth complex. $\mathrm{BiCl}_{3}(0.6297 \mathrm{~g}, 2 \mathrm{mmol})$ was added to a round bottom flask with $20 \mathrm{~mL}$ of 1,5-pentanediol. The solution was then degassed at $120{ }^{\circ} \mathrm{C}$. During heating, the $\mathrm{BiCl}_{3}$ was observed to dissolve to form a pale white cloudy solution. After 30 minutes, $20 \mathrm{~mL}$ of thioglycolic acid was added, upon which the solution turned yellow due to the thioligated complex.

Synthesis of TOP-Te solution. TOP-Te was prepared using the same method as that discussed in subsection 3.2.1. 
Synthesis of Bi2Te3-TGA. $6 \mathrm{~mL}$ of 0.5 M TOP-Te was injected into the thioligated bismuth complex, at which time the solutions turned black instantly. The reaction was held at $120{ }^{\circ} \mathrm{C}$ for 16 hours under $\mathrm{N}_{2}$. The solution was then cooled to room temperature naturally. During the cooling $10 \mathrm{~mL}$ of acetone was added. The nanoparticles were then washed several times by adding acetone (solvent) and isopropanol (antisolvent) followed by centrifugation for 5 minutes at 6000 RPM. The purified nanoparticles were then dried under vacuum and the fine powder was collected and stored in a vacuum desiccator. Nanocomposite pellets were then made using the dried nanoparticles and a 6 mm die.

\subsubsection{Characterisation and thermoelectric properties}

Powder XRD spectra were obtained for the samples capped with OLA, DDT, and TGA which are shown in Figure 3.5. The XRD spectra indicates a crystalline product is formed, which can be indexed to rhombohedral $\mathrm{Bi}_{2} \mathrm{Te}_{3}$. Using Scherrer's equations the crystallite sizes were estimated to be $12 \mathrm{~nm}, 23$ $\mathrm{nm}$, and $23 \mathrm{~nm}$ for DDT, OLA, and TGA capped $\mathrm{Bi}_{2} \mathrm{Te}_{3}$ respectively.

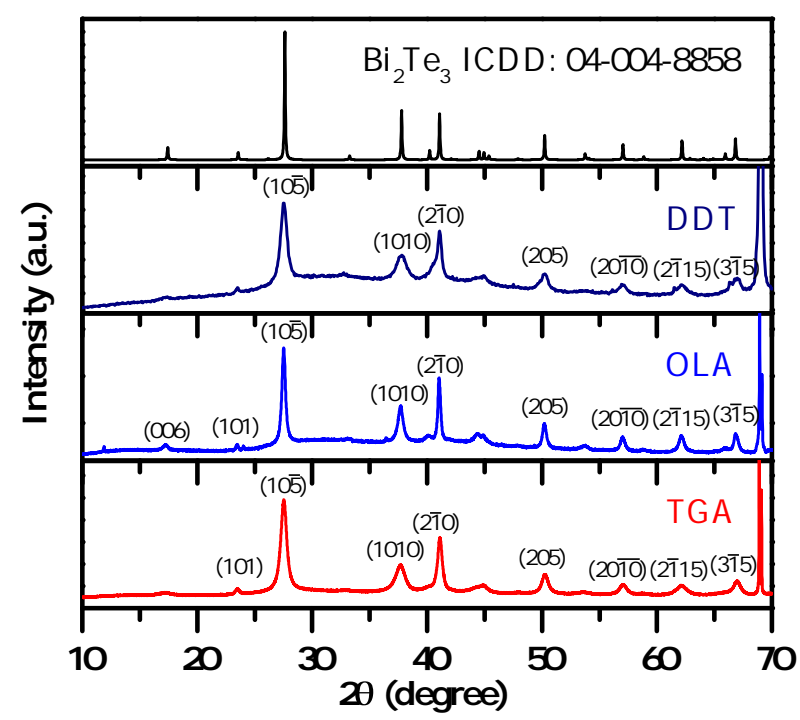

FIGURE 3.5: Powder XRD spectra of $\mathrm{Bi}_{2} \mathrm{Te}_{3}$ capped with OLA, DDT, and TGA. Note the peak at $69^{\circ}$ is due to the Si substrate.

TEM micrographs of DDT, OLA, and TGA caped bismuth telluride are shown in Figure 3.6, Figure 3.7, and Figure 3.8 respectively . The TEM images for $\mathrm{Bi}_{2} \mathrm{Te}_{3}-\mathrm{DDT}$ appears to have agglomerated nanoparticles consisting of thin nanoplates. The TEM images of $\mathrm{Bi}_{2} \mathrm{Te}_{3}-\mathrm{OLA}$ indicate a similar agglomerated nanoparticle consisting of thin nanoplates. Perhaps the nanoplates appear to be slightly thicker in this sample, which is consistent with the estimated 
(a)

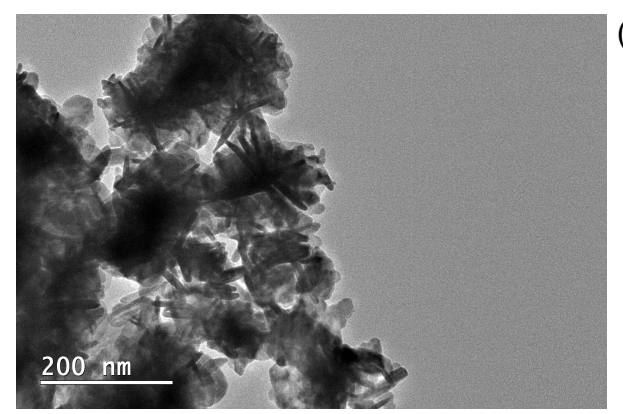

(c)

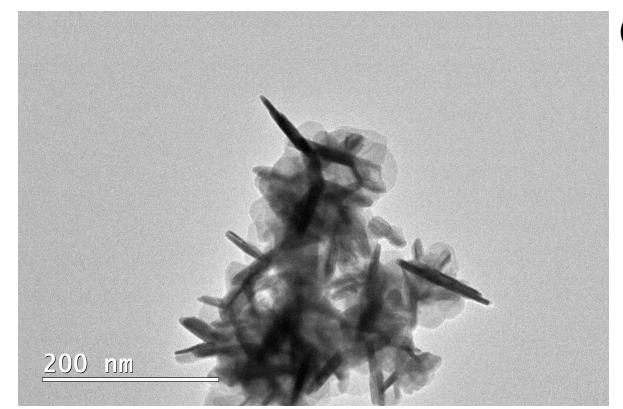

(b)

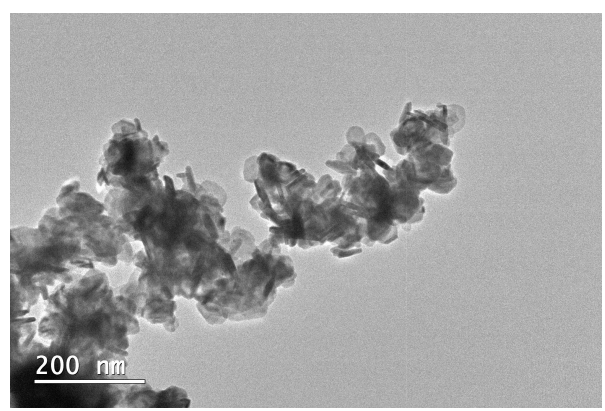

(d)

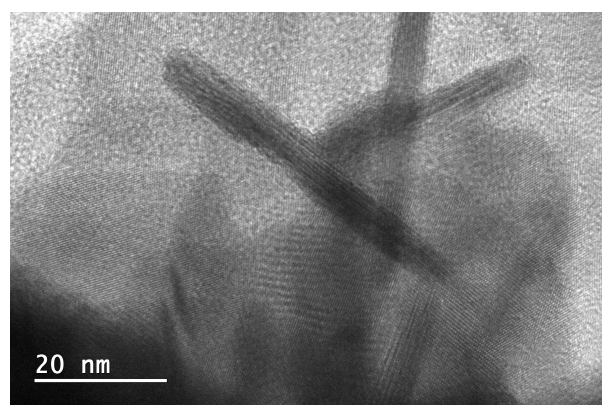

FIGURE 3.6: TEM micrographs of $\mathrm{Bi}_{2} \mathrm{Te}_{3}$ capped with DDT.

TABLE 3.3: Composition determined by TEM/EDS for $\mathrm{Bi}_{2} \mathrm{Te}_{3}$ capped with DDT, OLA, and TGA.

\begin{tabular}{ccc}
\hline \multirow{2}{*}{ Sample } & \multicolumn{2}{c}{ Normalized relative elemental composition } \\
\cline { 2 - 3 } & $\mathbf{B i}$ & Te \\
\hline $\mathrm{Bi}_{2} \mathrm{Te}_{3}-\mathrm{DDT}$ & 2.00 & 1.90 \\
$\mathrm{Bi}_{2} \mathrm{Te}_{3}-\mathrm{OLA}$ & 2.00 & 1.91 \\
$\mathrm{Bi}_{2} \mathrm{Te}_{3}-\mathrm{TGA}$ & 2.00 & 3.74 \\
\hline
\end{tabular}

crystallite size from XRD. The TEM images of $\mathrm{Bi}_{2} \mathrm{Te}_{3}-\mathrm{TGA}$ nanoparticles appear to be agglomerated and consist of thin nanoplates. In all three of these samples regardless of the capping ligand the nanostructures appear to have a similar morphology of agglomerated nanoplates. This morphology is typical of $\mathrm{Bi}_{2} \mathrm{Te}_{3}$ synthesised in solution $38,39,65,66$. The samples capped with DDT and OLA appear to be very similar to that produced at $100{ }^{\circ} \mathrm{C}$ without additional ligands. This suggests that oleic acid, DDT and OLA all have a similar effect on the growth and stabilization of $\mathrm{Bi}_{2} \mathrm{Te}_{3}$ nanoparticles synthesised via bismuth(III) oleate precursor.

The elemental composition determined from TEM/EDS for DDT, OLA and TGA capped $\mathrm{Bi}_{2} \mathrm{Te}_{3}$ nanostructures is shown in Table 3.3. The nanoparticles capped with DDT and OLA have a similar composition. The materials synthesised via bismuth oleate have a similar composition regardless of the capping ligand used. In this case the materials are $\mathrm{Bi}$ rich, due to the ease of $\mathrm{Bi}$ being incorporated between the hexagonal planes of rhombohedral $\mathrm{Bi}_{2} \mathrm{Te}_{3}{ }^{67}$. 
(a)

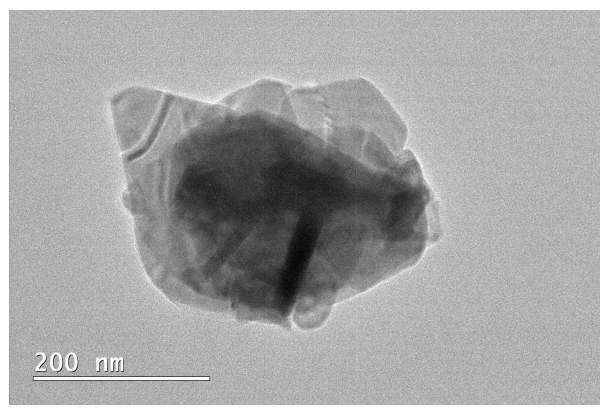

(c)

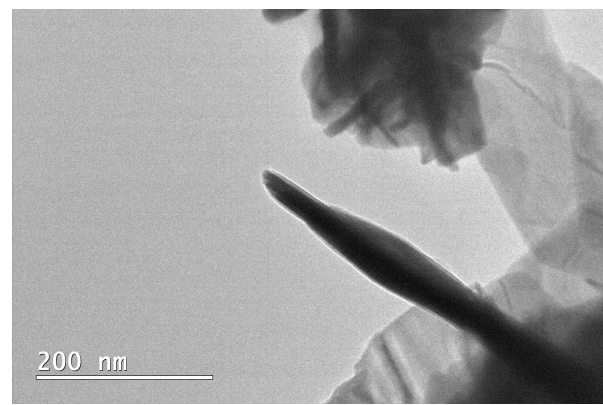

(b)

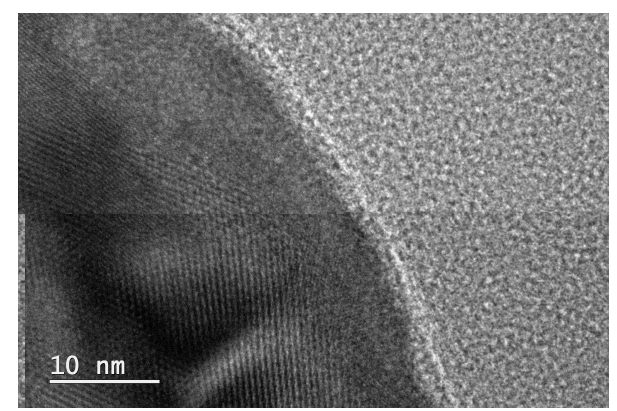

(d)

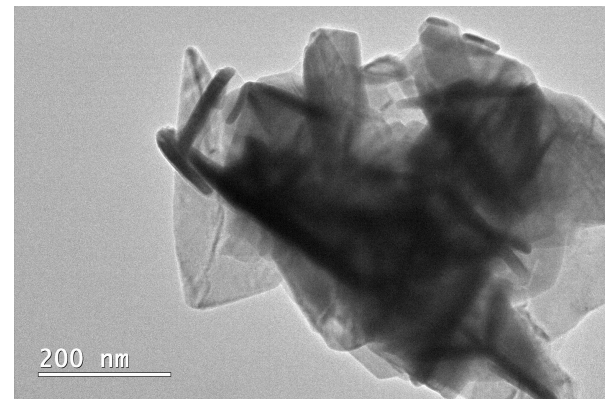

FIGURE 3.7: TEM micrographs of $\mathrm{Bi}_{2} \mathrm{Te}_{3}$ capped with OLA.

(a)

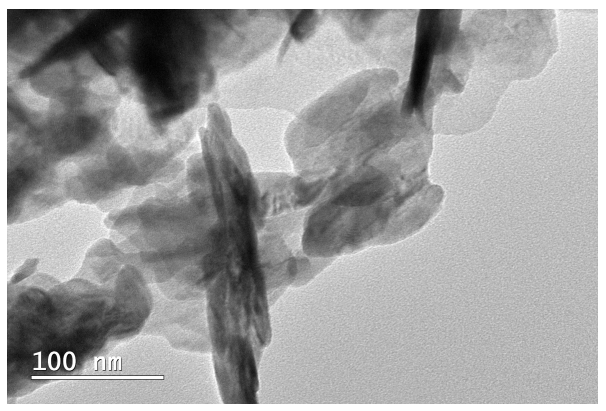

(c)

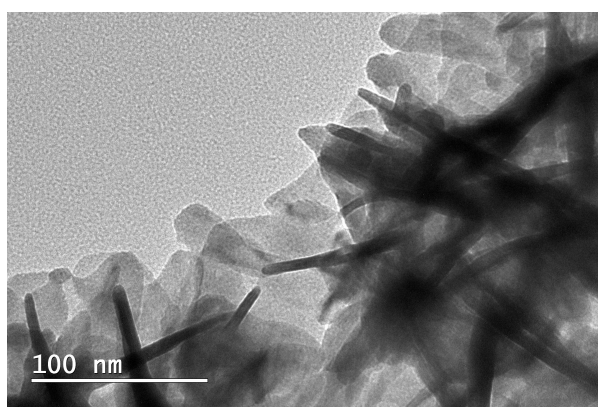

(b)

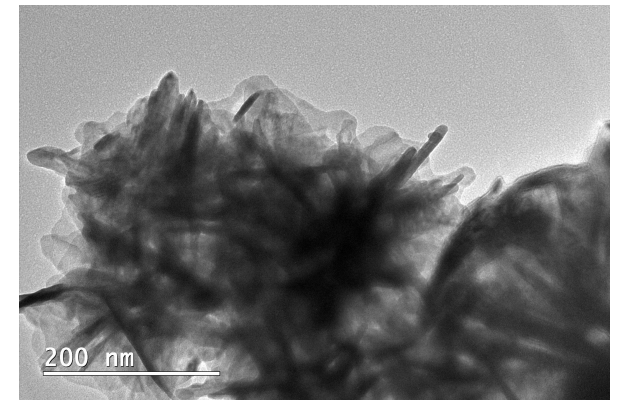

(d)

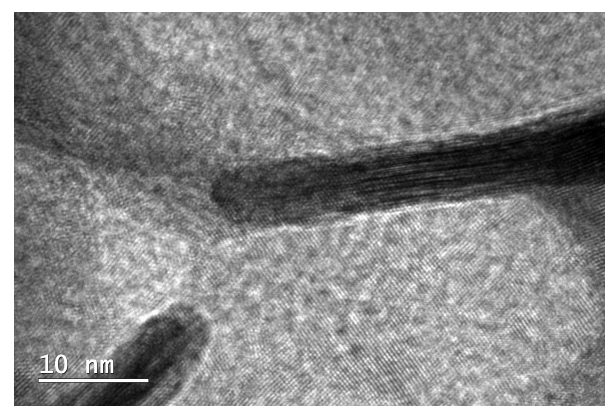

FIGURE 3.8: TEM micrographs of $\mathrm{Bi}_{2} \mathrm{Te}_{3}$ capped with TGA.

Interestingly the nanoparticles capped with TGA appear to have an excess of Te present, which is contrary to most $\mathrm{Bi}_{2} \mathrm{Te}_{3}$ nanomaterials which are typically Bi rich. It should be noted that the standard deviation for the Te composition in this sample was $3.74 \pm 1.2$ which is quite high and shows how inhomogeneous the sample is. If there is indeed an excess of Te present then perhaps decreasing the reaction time to several hours may yield stoichiometric $\mathrm{Bi}_{2} \mathrm{Te}_{3}$ nanostructures. 
(a)

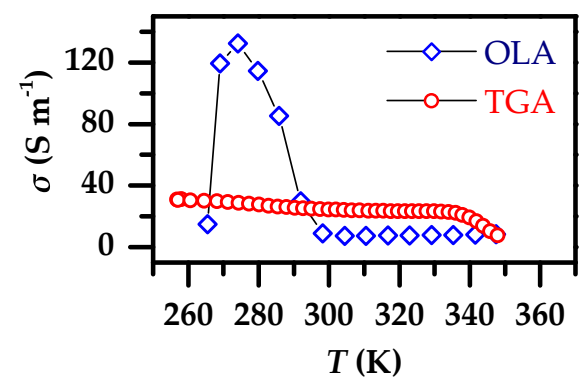

(c)

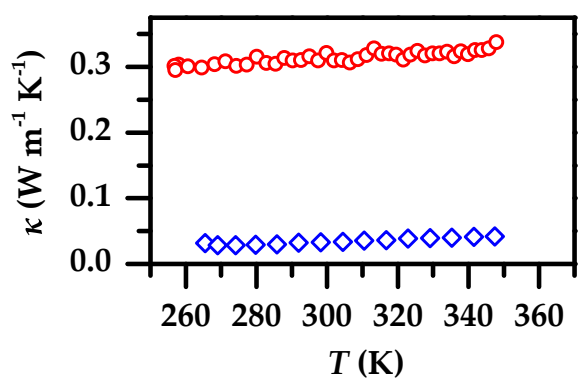

(b)

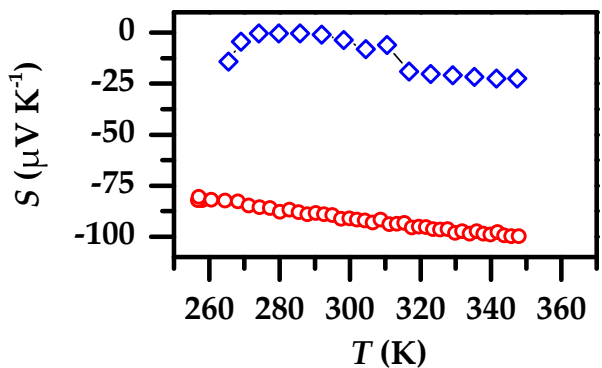

(d)

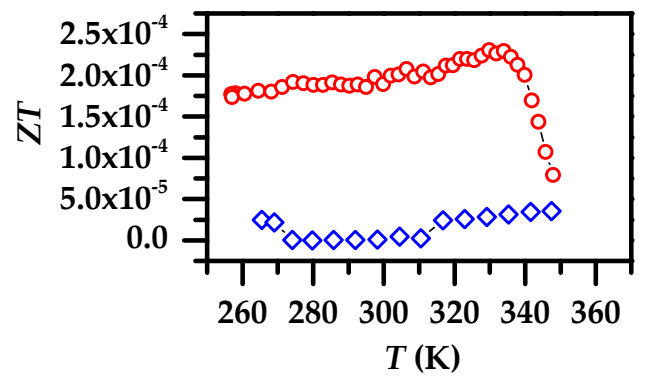

FIGURE 3.9: (a) Electrical conductivity, (b) thermopower, (c) thermal conductivity, and (d) figure of merit of $\mathrm{Bi}_{2} \mathrm{Te}_{3}$ capped with OLA and TGA.

The thermoelectric properties of $\mathrm{Bi}_{2} \mathrm{Te}_{3}$ capped with OLA and TGA are shown in Figure 3.9. In the case of the sample capped with OLA the low conductivity, along with the anomalous peak in the electrical conductivity and the low thermopower are indicative of some sort of contact issue occurring during the measurement. The conductivity in the TGA and OLA samples are still significantly lower than that of bulk $\mathrm{Bi}_{2} \mathrm{Te}_{3}$ or nanostructured $\mathrm{Bi}_{2} \mathrm{Te}_{3}$. At low temperatures the TGA sample appears to have higher conductivity than the sample synthesised at $100{ }^{\circ} \mathrm{C}$ via bismuth(III) oleate. This may be due to the shorter TGA ligand used, as the long chain oleic acid ligand used for the sample synthesised at $100{ }^{\circ} \mathrm{C}$ acts as an insulating barrier between nanoparticles. Interestingly the OLA sample appears to have a large peak in the electrical conductivity at $\sim 280 \mathrm{~K}$. The negative thermopower indicates the formation of n-type $\mathrm{Bi}_{2} \mathrm{Te}_{3}$, where the thermopower for the TGA capped sample is significantly higher than the OLA capped sample. The thermopower at $300 \mathrm{~K}$ for the TGA sample is $\sim 90 \mu \mathrm{V} \mathrm{K}^{-1}$ this is comparable to other nanostructured n-type $\mathrm{Bi}_{2} \mathrm{Te}_{3}{ }^{40}$. The higher absolute thermopower for the TGA capped sample relative to the OLA capped sample maybe due to sulphur doping from the TGA ligand, as the thermopower has been shown to have a high sensitivity to sulphur doping ${ }^{33}$. The thermal conductivity of both TGA and OLA capped nanostructures are both lower than bulk $\mathrm{Bi}_{2} \mathrm{Te}_{3}$ and nanostructured $\mathrm{Bi}_{2} \mathrm{Te}_{3}{ }^{2,33,40}$. The thermal conductivity at room temperature is $\sim 0.32$ and $\sim 0.03$ $\mathrm{W} \mathrm{m}{ }^{-1} \mathrm{~K}^{-1}$ for TGA and OLA capped $\mathrm{Bi}_{2} \mathrm{Te}_{3}$. The thermal conductivity of 
OLA capped $\mathrm{Bi}_{2} \mathrm{Te}_{3}$ is an order of magnitude lower than TGA capped $\mathrm{Bi}_{2} \mathrm{Te}_{3}$ and 2 orders of magnitude lower than bulk $\mathrm{Bi}_{2} \mathrm{Te}_{3}$.

\subsubsection{Ligand exchange}

During synthesis, surface coordinating ligands like oleic acid and oleylamine are needed to control the nanocrystal growth and stabilise nanoparticles in solution. These typical stabilising ligands are long chain organic molecules with coordinating functional groups, which act as insulators and block the charge transport between the nanocrystals. Research into the surface modification of surface ligands has mainly been focused on replacing the long chain ligands with shorter alky ligands, metal ligands, and or metal free chalcogenide ligands ${ }^{68-73}$. Ligand exchange using $S^{2-}$ and hydrazine has already been shown to be suitable for applications in solar cells and thermoelectrics ${ }^{72-74}$. Here we investigate the effects of a hydrazine and $S^{2-}$ ligand exchange on $\mathrm{Bi}_{2} \mathrm{Te}_{3}$ capped with DDT and OLA.

(a)

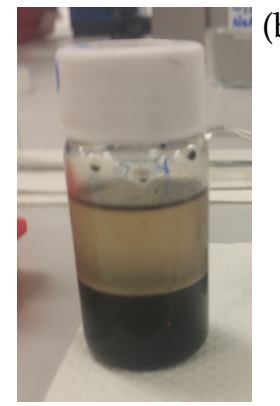

(b)

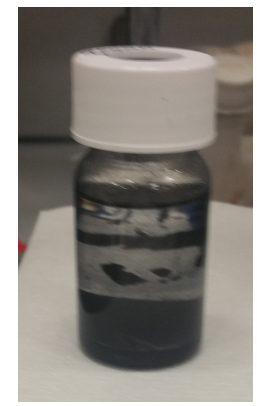

FIGURE 3.10: (a) Nanoparticles transferred from the toluene phase (top) into the formamide phase (bottom) after $\mathrm{S}^{2-}$ ligand exchange. (b) Nanoparticles precipitated out of solution after hydrazine ligand exchange.

$\mathrm{S}^{2-}$ ligand exchange. $\sim 400 \mathrm{mg}$ of $\mathrm{Bi}_{2} \mathrm{Te}_{3}$-OLA was dispersed in $8 \mathrm{~mL}$ of toluene. $8 \mathrm{~mL}$ of $1: 1(\mathrm{v} / \mathrm{v}) 10 \%\left(\mathrm{NH}_{4}\right)_{2} \mathrm{~S} /$ formamide was then added to the solution. The solution was then stirred overnight upon which the nanoparticles transfer from the toluene phase into the formamide phase as shown in Figure 3.10(a). The formamide layer was collected and washed 3 times with toluene.

Hydrazine ligand exchange. $\sim 450 \mathrm{mg}$ of $\mathrm{Bi}_{2} \mathrm{Te}_{3}-$ DDT was dispersed in 9 $\mathrm{mL}$ of toluene. $9 \mathrm{~mL}$ of 7:3 (v/v) acetonitrile/1 M hydrazine in THF was added. The solution was stirred vigorously overnight upon which the nanoparticles were observed to precipitate out of solution as shown in Figure 3.10(b). The nanoparticles were then washed several times with acetonitrile followed by 
centrifugation at 5000 RPM for 5 minutes. The nanoparticles were then dried under vacuum.

In order to measure the effect of the ligands on the charge transport properties, the conductivity of nanocomposite pellets were measured. Pellets were prepared using a $6 \mathrm{~mm}$ die for $\mathrm{Bi}_{2} \mathrm{Te}_{3}$ samples before and after ligand exchange treatment. The top and bottom of the pellets were then coated in silver paste and cured at $120^{\circ} \mathrm{C}$ for 30 minutes. The resistance was then measured using a multimeter and the dimensions of the pellet were measured using a digital vernier calliper. The electrical conductivity was then determined for each sample which are summarized in Table 3.4. We can see that after ligand exchange

TABLE 3.4: Conductivity of nanocomposite $\mathrm{Bi}_{2} \mathrm{Te}_{3}$ pellets before and after ligand exchange with hydrazine and $\mathrm{S}^{2-}$.

\begin{tabular}{lr}
\hline Sample & Conductivity $\left(\mathrm{S} \mathrm{m}^{-1}\right)$ \\
\hline $\mathrm{Bi}_{2} \mathrm{Te}_{3}-\mathrm{DDT}$ as-synthesized & $0.34 \pm 0.01$ \\
$\mathrm{Bi}_{2} \mathrm{Te}_{3}-\mathrm{DDT}$ after hydrazine LE & $6 \pm 1$ \\
$\mathrm{Bi}_{2} \mathrm{TE}_{3}-\mathrm{OLA}$ as-synthesized & $16 \pm 1$ \\
$\mathrm{Bi}_{2} \mathrm{Te}_{3}-\mathrm{OLA}$ after $\mathrm{S}^{2-} \mathrm{LE}$ & $27 \pm 2$ \\
\hline
\end{tabular}

with both hydrazine and $\mathrm{S}^{2-}$, both the nanocomposite pellets have increased in conductivity with hydrazine increasing by an order of magnitude relative to the as-synthesized pellet. This hydrazine treatment shows the potential for increasing the conductivity of nanocomposite pellets and further enhancing the figure of merit.

\subsection{Nanostructured $\mathrm{Bi}_{2} \mathrm{Te}_{3}$ Synthesized via Bismuth Nanoparticles}

\subsubsection{Synthesis via Bi nanoparticle}

Our initials experiments showed a large distribution in the size and morphology of the $\mathrm{Bi}_{2} \mathrm{Te}_{3}$ nanostructures which shows the difficulty in solution processed bismuth telluride, which is attributed to the high reactivity of tellurium with bismuth salts. In order to try synthesize $\mathrm{Bi}_{2} \mathrm{Te}_{3}$ nanostructures with a more monodisperse and sub-50 nm size a method developed by Scheele et al. (2009) was used ${ }^{40}$. In this reaction $\mathrm{Bi}_{2} \mathrm{Te}_{3}$ nanoparticles are synthesized using a bismuth nanoparticle and TOP-Te precursor. The use of a Bi nanoparticle precursor should lead to more monodisperse sub-50 nm 
$\mathrm{Bi}_{2} \mathrm{Te}_{3}$ nanoparticles ${ }^{40}$. The following reactions are carried out using standard Schlenk line techniques under a vacuum/nitrogen.

Synthesis of Bi nanoparticle precursor. In a sealed 3-neck flask with attached condenser and hose adapter all connected to the Schlenk line, bismuth(III) acetate $(0.7722 \mathrm{~g}, 2 \mathrm{mmol})$ was added to $20 \mathrm{~mL}$ of 1 -dodecanethiol (stabilising ligand) with gentle stirring and degassed at $60{ }^{\circ} \mathrm{C}$ for 1 hour. In a second 3-neck flask $40 \mathrm{~mL}$ of oleylamine was degassed at $60^{\circ} \mathrm{C}$ for 1 hour. Both solutions were then purged with nitrogen and the oleylamine was then injected into the bismuth solution. The reaction was then kept at $60{ }^{\circ} \mathrm{C}$ for 24 hours under nitrogen. For characterisation a small aliquot $(1 \mathrm{~mL})$ was collected and washed several times with chloroform (solvent) and ethanol (antisolvent) followed by centrifugation for 5 minutes at 5000 RPM.

Synthesis of TOP-Te solution. Using a glove box under a nitrogen atmosphere, Te powder $(0.3828 \mathrm{~g}, 3 \mathrm{mmol})$, octadecylphosphonic acid (30 mg), and $6 \mathrm{~mL}$ of TOP were added to a round bottom flask. The solution was then transferred onto a Schlenk line under nitrogen and heated to $180{ }^{\circ} \mathrm{C}$ in 50 ${ }^{\circ} \mathrm{C} / 30$ mins increments with vigorous stirring. After 1 hour at $180{ }^{\circ} \mathrm{C}$ the Te was observed to dissolve and form a pale yellow solution. The solution was then cooled to the required injection temperature $\left(60\right.$ or $\left.120^{\circ} \mathrm{C}\right)$.

Synthesis of $\mathbf{B i}_{2} \mathbf{T e}_{3}$ Nanoparticles. The TOP-Te solution was then injected into the bismuth nanoparticle precursor solution and heated to $120{ }^{\circ} \mathrm{C}$ (or 60 ${ }^{\circ} \mathrm{C}$ ) for 3 days under nitrogen with vigorous stirring. The solution was then cooled down to room temperature naturally and transferred to a centrifuge tube. Ethanol (25 vol\%) was then added and centrifuged for 5 minutes at 5000 RPM. The nanoparticles were then redispersed in chloroform (solvent) and the washing step with ethanol (antisolvent) is repeated several times. The nanoparticles were then dried under vacuum and the fine powder was collected and stored in a vacuum desiccator.

Ligand Exchange with Hydrazine. $\sim 600 \mathrm{mg}$ of $\mathrm{Bi}_{2} \mathrm{Te}_{3}$ was dispersed in $12 \mathrm{~mL}$ of toluene. $4 \mathrm{~mL}$ of $1 \mathrm{M}$ hydrazine in THF and $8 \mathrm{~mL}$ of acetonitrile were then added and the solution was stirred vigorously overnight. The nanoparticles were then collected using a centrifuge at 5000 RPM for 5 minutes. The nanoparticles were then washed several times with acetonitrile followed by centrifugation. The nanoparticles were then dried on a vacuum and the powder was collected and stored in a vacuum desicator. Nanocomposite pellets were then prepared using a $5 \mathrm{~mm}$ pellet die. Pellets typically had a thickness of $\sim 2.5 \mathrm{~mm}$. 


\subsubsection{Characterisation and thermoelectric properties}

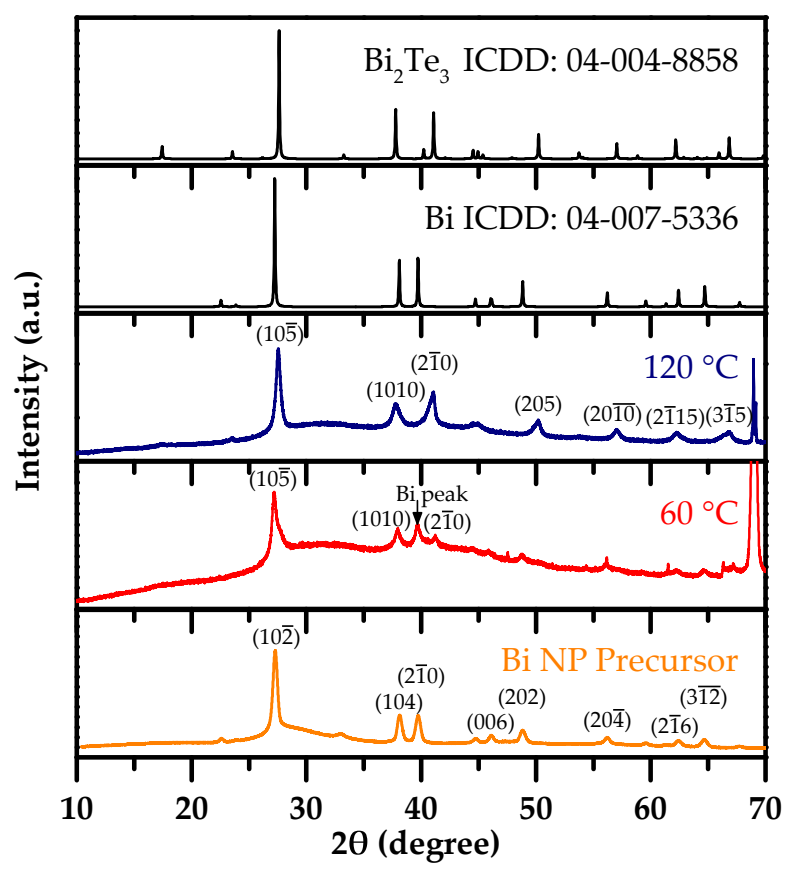

FIGURE 3.11: Powder XRD spectra of $\mathrm{Bi}_{2} \mathrm{Te}_{3}$ synthesised at 60 ${ }^{\circ} \mathrm{C}$ and $120^{\circ} \mathrm{C}$ via Bi nanoparticles. Note the peak at $69^{\circ}$ is due to the Si substrate.

Powder XRD spectra were obtained for the Bi nanoparticle precursor and the samples synthesised at $60{ }^{\circ} \mathrm{C}$ and $120{ }^{\circ} \mathrm{C}$ and are shown in Figure 3.11. The spectra indicates a crystalline product is formed which can be indexed to rhombohedral bismuth and rhombohedral $\mathrm{Bi}_{2} \mathrm{Te}_{3}$ respectively. The FWHM indicates the increase in crystallite size with increasing reaction temperature for $\mathrm{Bi}_{2} \mathrm{Te}_{3}$ nanoparticles. Estimation of the crystallite size using Scherrer's equation gives 19,11 and $17 \mathrm{~nm}$ for the $\mathrm{Bi} \mathrm{NP}$ precursor, $\mathrm{Bi}_{2} \mathrm{Te}_{3}$ synthesised at $60{ }^{\circ} \mathrm{C}$ and $120{ }^{\circ} \mathrm{C}$ respectively. The XRD patterns show the reaction carried out at lower temperature still has a significant Bi phase present as indicated by the $(20 \overline{1})$ rhombohedral Bi peak present in the spectrum for $\mathrm{Bi}_{2} \mathrm{Te}_{3}$ synthesised at $60{ }^{\circ} \mathrm{C}$. Increasing the reaction temperature appears to result in the Bi peak dissapearing.

TEM micrographs and the particle distribution of the Bi nanoparticle precursor are shown in Figure 3.12. The TEM micrographs indicate the formation of sub-60 nm Bi nanoparticle. The nanoparticles appear to be roughly spherical and the size distribution is reasonable narrow with an average particle size of $41 \mathrm{~nm}$. These nanoparticles are significantly larger than the sub-10 nm particles reported by Scheele et al. (2009) produced using a similar method ${ }^{40}$. 
(a)

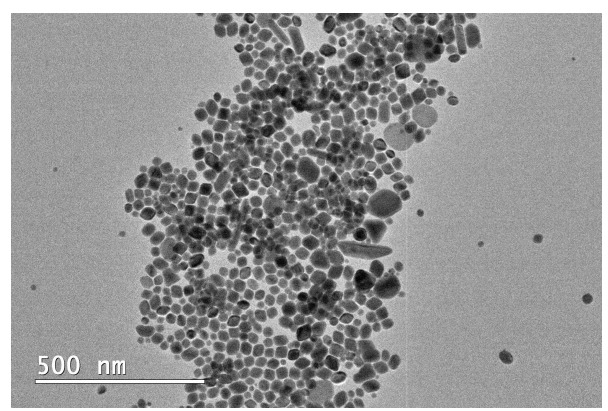

(b)

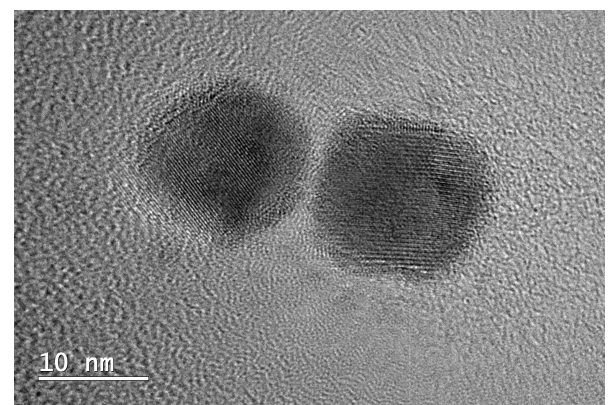

(c)

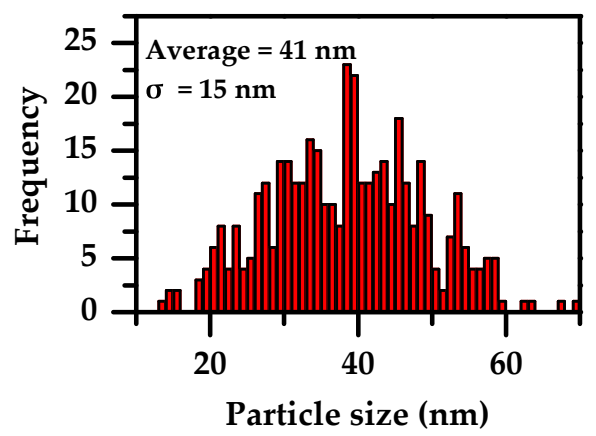

FIGURE 3.12: (a)(b) TEM micrographs and (c) particle distribution of bismuth nanoparticle precursor.

(a)

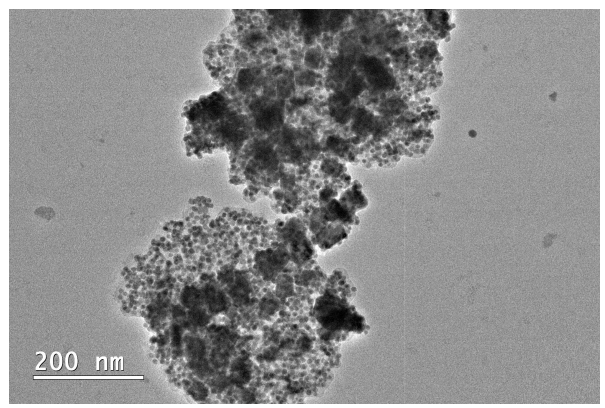

(c)

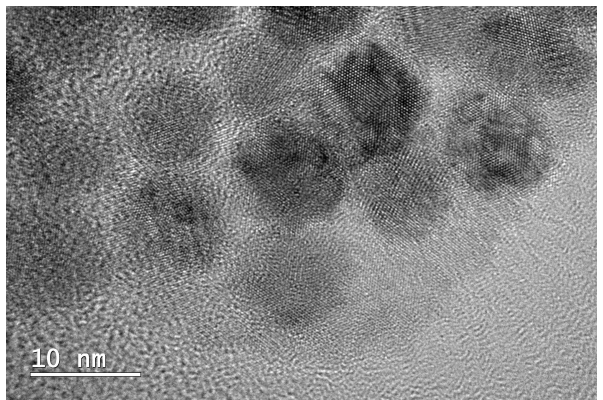

(b)

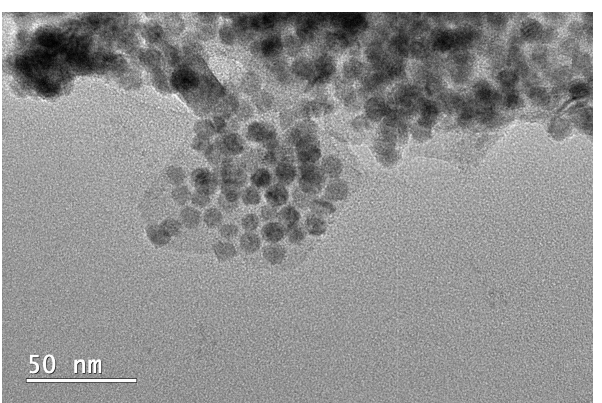

(d)

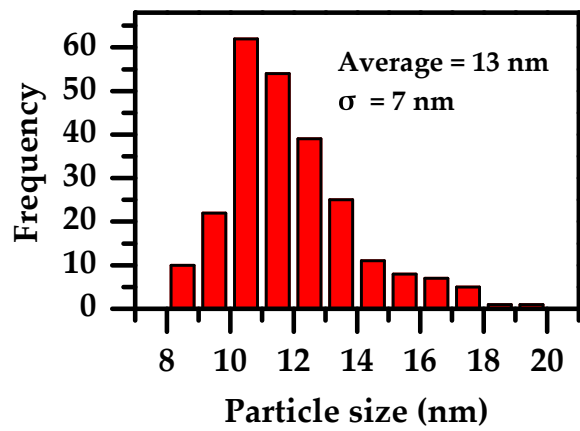

FIGURE 3.13: (a)(b)(c) TEM micrographs and (d) particle distribution of $\mathrm{Bi}_{2} \mathrm{Te}_{3}$ synthesised at $60^{\circ} \mathrm{C}$ via bismuth nanoparticles.

TEM micrographs and particle distribution of $\mathrm{Bi}_{2} \mathrm{Te}_{3}$ synthesised at 60 ${ }^{\circ} \mathrm{C}$ are shown in Figure 3.13. The TEM micrographs show small spherical particles that have agglomerated. Analysis of the particles that are not agglomerated shows a narrow distribution with an average particle size of 13 
(a)

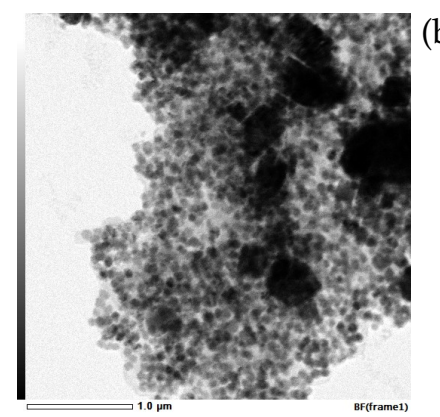

(b)

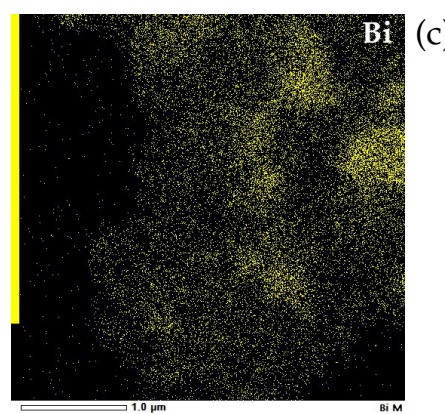

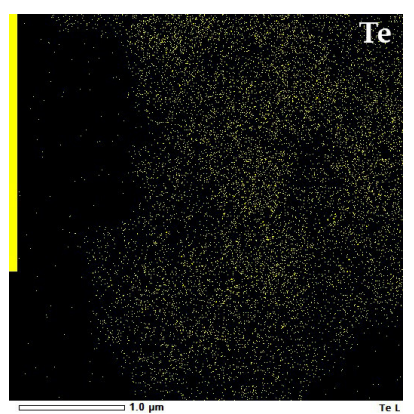

FIGURE 3.14: STEM/EDS elemental map of $\mathrm{Bi}_{2} \mathrm{Te}_{3}$ synthesised at $60{ }^{\circ} \mathrm{C}$ via bismuth nanoparticles. (a) STEM-BF image (b) Bi map (c) Te map, the scale bar is $1 \mu \mathrm{m}$.

(a)

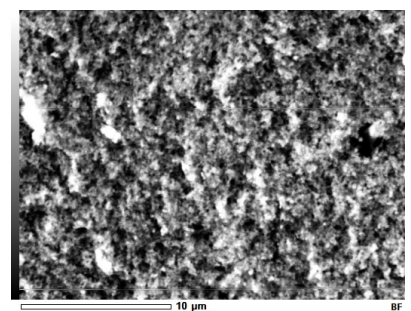

(b)

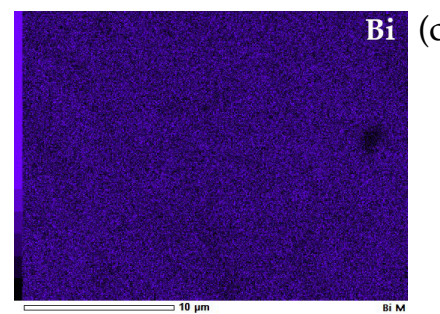

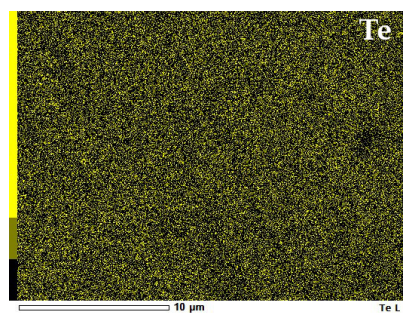

FIGURE 3.15: SEM/EDS elemental map of nanocomposite pellet prepared from $\mathrm{Bi}_{2} \mathrm{Te}_{3}$ synthesised at $60^{\circ} \mathrm{C}$ via bismuth nanoparticles. (a) SEM-SEI image (b) Bi map (c) Te map, the scale bar is $10 \mu \mathrm{m}$.

nm. The size of the $\mathrm{Bi}_{2} \mathrm{Te}_{3}$ nanoparticles is significantly smaller than the $\mathrm{Bi}$ nanoparticle precursor. $\mathrm{The}^{\mathrm{Bi}} \mathrm{Te}_{3}$ nanoparticles are formed by tellurizing the Bi nanoparticles, which should result in the $\mathrm{Bi}_{2} \mathrm{Te}_{3}$ nanoparticles being larger than the Bi precursor ${ }^{40}$. In our case the observed differences may be due to the centrifugal processing step being size selective of the larger Bi nanoparticles, as the smaller particles are less likely to precipitate out of solution at low RPMs compared to the larger particles. STEM/EDS elemental maps for $\mathrm{Bi}_{2} \mathrm{Te}_{3}$ synthesised at $60^{\circ} \mathrm{C}$ are shown in Figure 3.14. We can see that the Te atoms are uniformly distributed across the sample, however the Bi map appears to have higher concentration areas of $\mathrm{Bi}$. These areas correspond to the aggregated/large particles in the STEM image. This suggest the large aggregated particles are $\mathrm{Bi}$ while the sub-15 nm particles correspond to $\mathrm{Bi}_{2} \mathrm{Te}_{3}$. The presence of the larger Bi particles may be from unreacted Bi nanoparticle precursors. SEM/EDS elemental maps for nanocomposite pellets made from $\mathrm{Bi}_{2} \mathrm{Te}_{3}$ synthesized at $60{ }^{\circ} \mathrm{C}$ are shown in Figure 3.15. The SEM-SEI image indicates the presence of compacted nanoparticles. The elemental map shows a uniform distribution of $\mathrm{Bi}$ and Te atoms. The elemental composition is summarized in Table 3.5, which indicates that there is an excess of Bi relative to Te. This suggests that we still have unreacted Bi nanoparticles present, 
TABLE 3.5: Composition determined by SEM/EDS for nanocomposite pellet of $\mathrm{Bi}_{2} \mathrm{Te}_{3}$ synthesised at $60^{\circ} \mathrm{C}$ via bismuth nanoparticles.

\begin{tabular}{ccc}
\hline \multirow{2}{*}{ Reference image } & \multicolumn{2}{c}{ Normalized relative elemental composition } \\
\cline { 2 - 3 } & $\mathbf{B i}$ & $\mathbf{T e}$ \\
\hline 1a-x5k & 2.00 & 1.19 \\
1b-x5k & 2.00 & 1.18 \\
1c-x5k & 2.00 & 1.15 \\
1d-x5k & 2.00 & 1.15 \\
1d-x5k & 2.00 & 1.17 \\
\cline { 2 - 3 } & $\sigma$ for Te composition & 0.01 \\
\hline Average & $\mathbf{2 . 0 0}$ & $\mathbf{1 . 1 7}$ \\
\hline
\end{tabular}

(a)

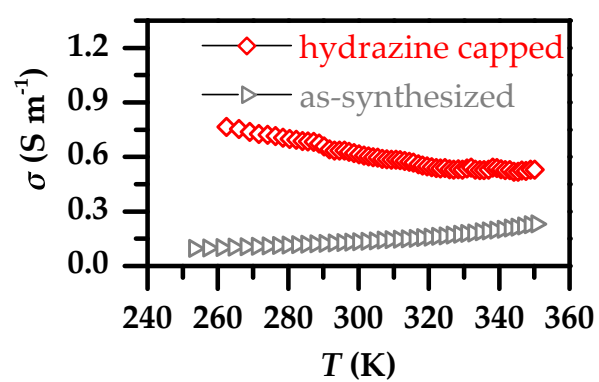

(c)

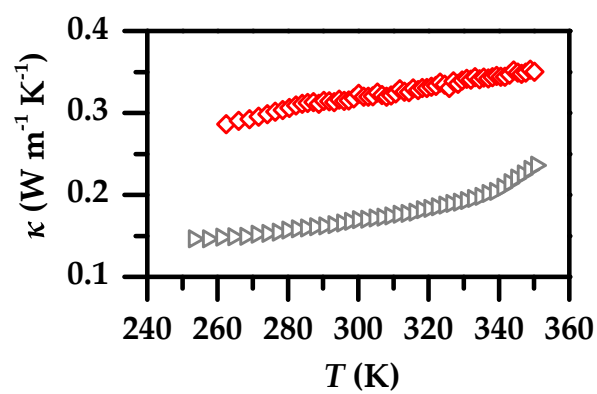

(b)

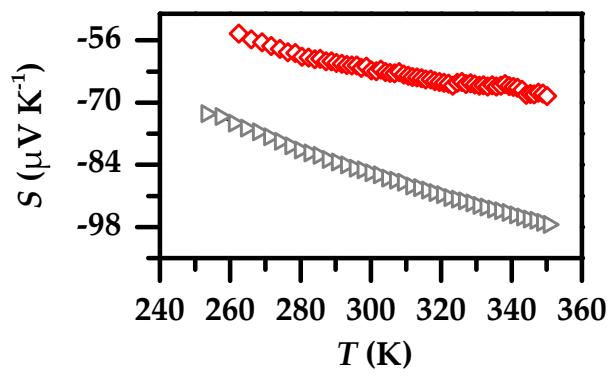

(d)

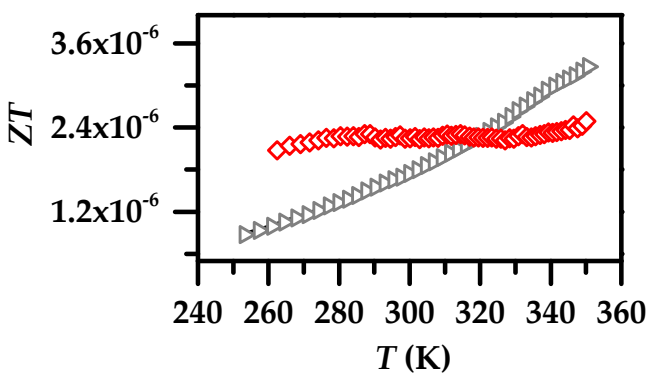

FIGURE 3.16: (a) Electrical conductivity, (b) thermopower, (c) thermal conductivity, and (d) figure of merit of as-synthesized and hydrazine capped $\mathrm{Bi}_{2} \mathrm{Te}_{3}$ synthesized at $60^{\circ} \mathrm{C}$ via bismuth nanoparticles.

which agrees with the observation of a Bi peak in the XRD spectra and the STEM/EDS elemental map showing a high concentration of Bi nanoparticles.

The thermoelectric properties for nanocomposite pellets of $\mathrm{Bi}_{2} \mathrm{Te}_{3}$ synthesised at $60{ }^{\circ} \mathrm{C}$ before and after ligand exchange are shown in 3.16. We can see that the electrical conductivity has increased after the ligand exchange treatment with hydrazine. The electrical conductivity at $300 \mathrm{~K}$ is $\sim 0.15 \mathrm{Sm}^{-1}$ and $\sim 0.61 \mathrm{~S} \mathrm{~m}^{-1}$ for the as-synthesised sample and the hydrazine treated 
sample. Although the ligand exchange provided a 4 times increase in the conductivity the conductivity is still significantly low compared to other nanostructured $\mathrm{Bi}_{2} \mathrm{Te}_{3}$ materials which have a conductivity between 70000-130000 $\mathrm{Sm}^{-1} 33,38,40,67$. The low electrical conductivity relative to other $\mathrm{Bi}_{2} \mathrm{Te}_{3}$ nanostructured materials is due to the fact that no post annealing or hot pressing step such as spark plasma sintering was done on the nanocomposite pellets, which is known to significantly increase the electrical conductivity ${ }^{75}$. The thermopower of the as-synthesised sample is lower than hydrazine treated sample. The absolute thermopower at $300 \mathrm{~K}$ is $\sim 85 \mu \mathrm{V} \mathrm{K}^{-1}$ and $\sim 60 \mu \mathrm{V} \mathrm{K}{ }^{-1}$ for the as-synthesised and hydrazine treated sample, which is comparable to other nanostructured $\mathrm{Bi}_{2} \mathrm{Te}_{3}$ materials ${ }^{38,40}$. The thermal conductivity has increased slightly after the hydrazine ligand exchange. At room temperature the thermal conductivity is $\sim 0.17$ and $\sim 0.32 \mathrm{~W} \mathrm{~m}^{-1} \mathrm{~K}^{-1}$ for the as-synthesised and hydrazine treated sample respectively. This is significantly lower than bulk $\mathrm{Bi}_{2} \mathrm{Te}_{3}$ and is still low for $\mathrm{Bi}_{2} \mathrm{Te}_{3}$ nanostructured materials ${ }^{2,33,38,40,67}$. Overall the figure of merit is still low and no significant improvements were made for the sample after hydrazine ligand exchange.

TEM micrographs and particle distribution for $\mathrm{Bi}_{2} \mathrm{Te}_{3}$ synthesised at 120 ${ }^{\circ} \mathrm{C}$ are shown in Figure 3.17. The majority of the nanoparticles appear to have agglomerated. The agglomerated materials consist of sub- $100 \mathrm{~nm}$ rhomboid shaped nanoparticles. The TEM micrographs also show a small fraction of well dispersed spherical nanoparticles which is most likely due to unreacted Bi nanoparticles precursor. Note the size distribution was only counted for particles with a well defined shape. This inherently means the large aggregated particles were not counted which may alter the overall particle distribution. The average particle size of our material is $36 \mathrm{~nm}$. In similar reactions carried out by Scheele et al. (2009), they stated that increasing the reaction temperature from $60^{\circ} \mathrm{C}$ to $110^{\circ} \mathrm{C}$ did not result in a significant change in the particle size however the particles were observed to change from almost spherical to rhombohedral structure ${ }^{40}$. The differences in the particle size of our materials and that reported by Scheele et al. (2009) may be due to the increased reaction time of our material which were synthesised for 3 days instead of $18 \mathrm{hrs}^{40}$. It is also possible the differences in the size of our bismuth nanoparticle precursor may have also had an affect. The increased size and morphology of the nanoparticles with increased temperature suggests that the Te atoms are indeed tellurizing the Bi nanoparticle precursor. STEM/EDS elemental maps of $\mathrm{Bi}_{2} \mathrm{Te}_{3}$ synthesised at $120{ }^{\circ} \mathrm{C}$ are shown in Figure 3.18. The elemental map indicates that the aggregated rhomboid particles have 
(a)

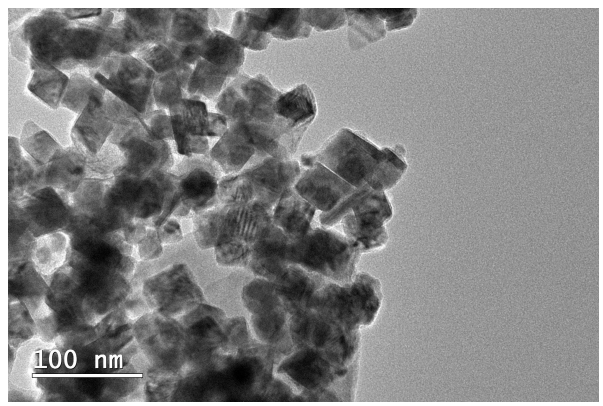

(c)

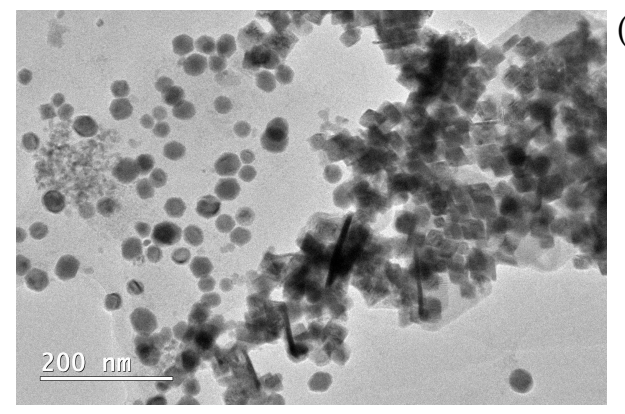

(b)

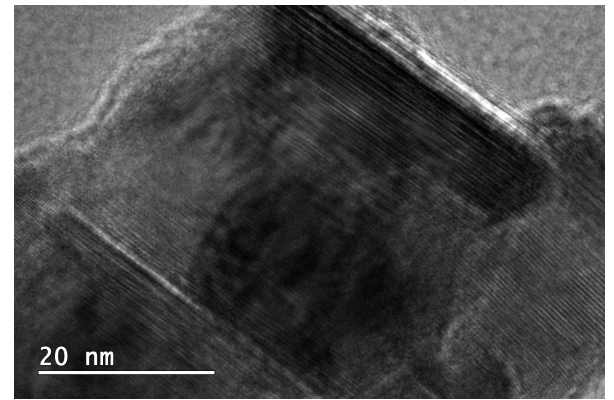

(d)

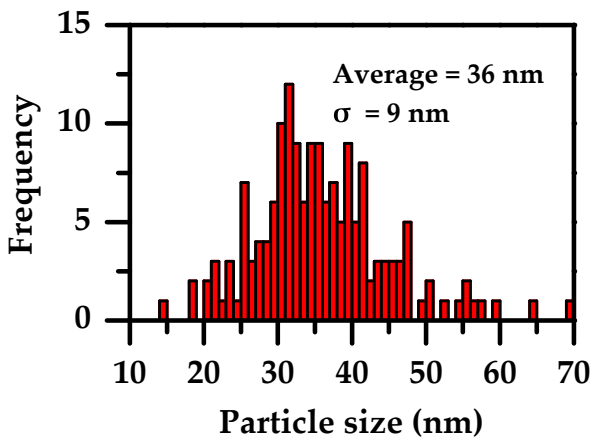

FIGURE 3.17: (a)(b)(c) TEM micrographs and (d) particle distribution of $\mathrm{Bi}_{2} \mathrm{Te}_{3}$ synthesised at $120^{\circ} \mathrm{C}$ via bismuth nanoparticles.

(a)

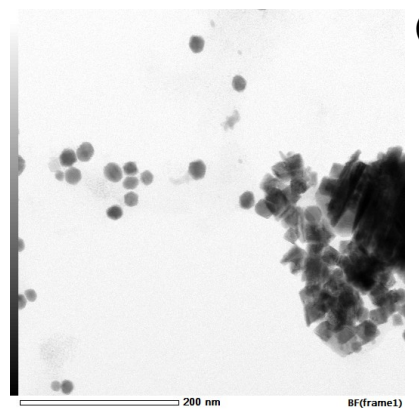

(b)

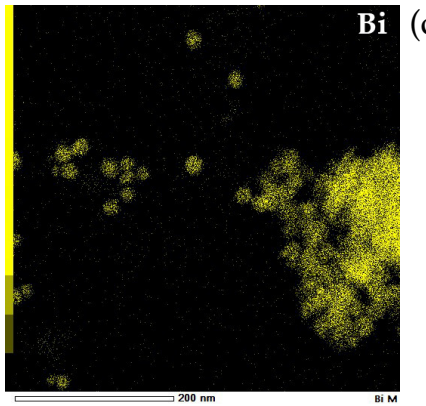

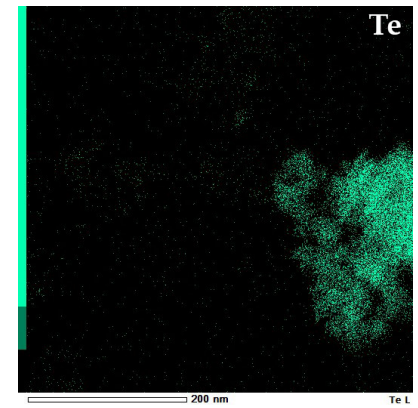

FIGURE 3.18: STEM/EDS map of $\mathrm{Bi}_{2} \mathrm{Te}_{3}$ synthesised at $120^{\circ} \mathrm{C}$ via bismuth nanoparticles. (a) STEM-BF image (b) Bi map (c) Te map, the scale bar is $200 \mathrm{~nm}$.

(a)

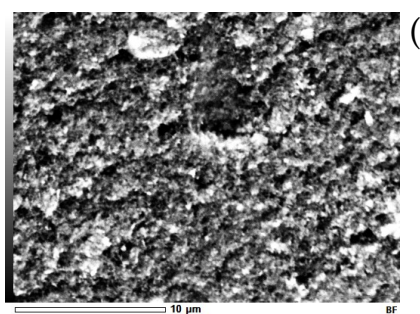

(b)

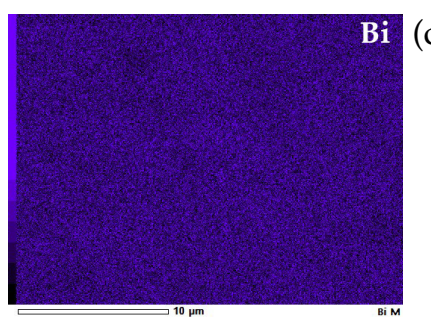

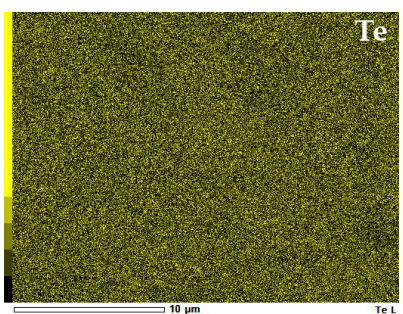

FIGURE 3.19: SEM/EDS elemental map of nanocomposite pellet prepared from $\mathrm{Bi}_{2} \mathrm{Te}_{3}$ synthesised at $120^{\circ} \mathrm{C}$ via bismuth nanoparticles. (a) SEM-SEI image (b) Bi map (c) Te map, the scale bar is $10 \mu \mathrm{m}$. 
TABLE 3.6: Composition determined by SEM/EDS for nanocomposite pellet of $\mathrm{Bi}_{2} \mathrm{Te}_{3}$ synthesised at $120^{\circ} \mathrm{C}$ via bismuth nanoparticles.

\begin{tabular}{ccc}
\hline \multirow{2}{*}{ Reference image } & \multicolumn{2}{c}{ Normalized relative elemental composition } \\
\cline { 2 - 3 } & $\mathbf{B i}$ & $\mathbf{T e}$ \\
\hline 1a-x5k & 2.00 & 2.55 \\
$1 \mathrm{~b}-\mathrm{x} 5 \mathrm{k}$ & 2.00 & 2.50 \\
$1 \mathrm{c}-\mathrm{x} 5 \mathrm{k}$ & 2.00 & 2.48 \\
$1 \mathrm{~d}-\mathrm{x} 5 \mathrm{k}$ & 2.00 & 2.57 \\
$1 \mathrm{e}-\mathrm{x} 5 \mathrm{k}$ & 2.00 & 2.50 \\
& $\sigma$ for Te composition & 0.04 \\
\hline Average & $\mathbf{2 . 0 0}$ & $\mathbf{2 . 5 2}$ \\
\hline
\end{tabular}

a uniform distribution of $\mathrm{Bi}$ and Te. However the spherically dispersed nanoparticles appear to consist of only Bi. This suggests the presence of unreacted Bi nanoparticles. SEM/EDS elemental maps for a nanocomposite pellet of $\mathrm{Bi}_{2} \mathrm{Te}_{3}$ synthesised at $120^{\circ} \mathrm{C}$ are shown in Figure 3.19. The SEM-SEI image indicates the presence of compacted nanoparticles. The elemental map shows a uniform distribution of $\mathrm{Bi}$ and Te across the pellet. The elemental composition is summarized in Table 3.6. We can see that the increase in the reaction temperature has resulted in a decrease in the Bi phase present. There is still an excess of Bi present however this is to be expected for materials synthesised via Bi nanoparticles ${ }^{40}$.

The thermoelectric properties of nanocomposite pellets of $\mathrm{Bi}_{2} \mathrm{Te}_{3}$ synthesised at $120^{\circ} \mathrm{C}$ with and without ligand exchange are shown in Figure 3.20. At $300 \mathrm{~K}$ the electrical conductivity was $\sim 7.4 \mathrm{Sm}^{-1}$ and $\sim 13.1 \mathrm{~S} \mathrm{~m}^{-1}$ for the as-synthesized and hydrazine capped sample respectively. After the ligand exchange with hydrazine the conductivity has double however this is still very low compared to other nanostructured $\mathrm{Bi}_{2} \mathrm{Te}_{3}$ thermoelectric materials $\mathrm{s}^{33,38,40}$. The absolute thermopower at $300 \mathrm{~K}$ was $\sim 99 \mu \mathrm{V} \mathrm{K}^{-1}$ and $\sim 94 \mu \mathrm{VK}^{-1}$ for the as-synthesized and hydrazine capped sample. This is comparable to other nanostructured $\mathrm{Bi}_{2} \mathrm{Te}_{3}$ materials and higher than the $\sim 60 \mu \mathrm{V} \mathrm{K}{ }^{-1}$ reported by Scheele et al. (2009) $33,38,40$. The thermal conductivity of our nanoparticles at $300 \mathrm{~K}$ was $\sim 0.36 \mathrm{~W} \mathrm{~m}^{-1} \mathrm{~K}^{-1}$ and $\sim 0.38 \mathrm{~W} \mathrm{~m}^{-1} \mathrm{~K}^{-1}$ for the as-synthesized and hydrazine capped sample respectively, which is significantly lower than bulk $\mathrm{Bi}_{2} \mathrm{Te}_{3}$ and even lower than the $\sim 0.8 \mathrm{~W} \mathrm{~m}^{-1} \mathrm{~K}^{-1}$ reported by Scheele et al. (2009) for $\mathrm{Bi}_{2} \mathrm{Te}_{3}$ nanoparticles ${ }^{40}$. The dimensionless figure of merit at $300 \mathrm{~K}$ was $5 \times 10^{-5}$ and $1 \times 10^{-4}$ for the as-synthesized and hydrazine capped 
sample. We can see that the hydrazine ligand exchange treatment has resulted in a doubling of the figure of merit. This doubling in the figure of merit can be attributed to the increased electrical conductivity of the hydrazine treated sample while the thermopower and thermal conductivity are largely unaffected by the ligand exchange. This shows great potential for using a hydrazine ligand exchange to improve the figure of merit of nanostructured materials.

(a)

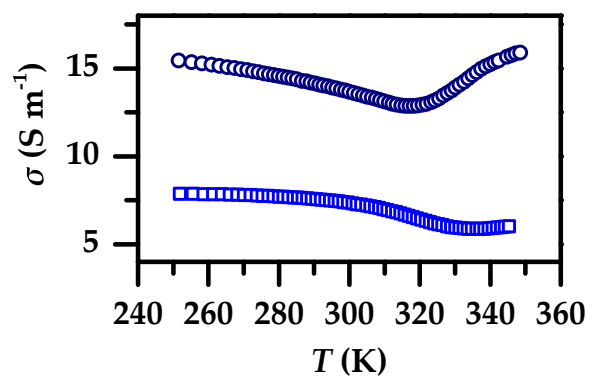

(c)

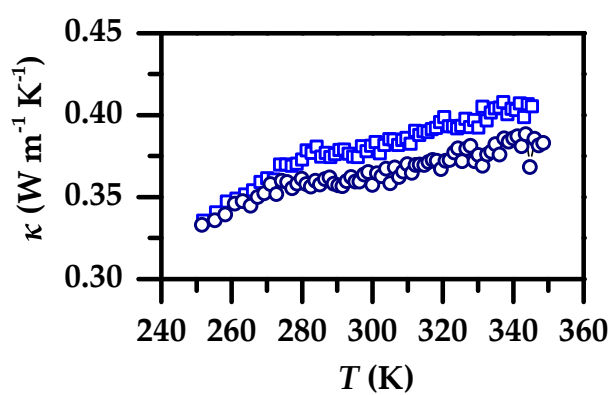

(b)

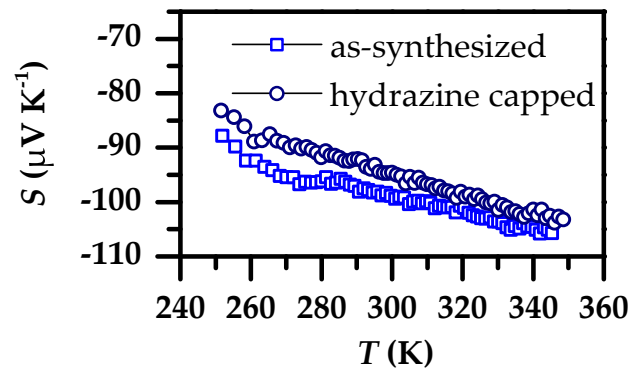

(d)

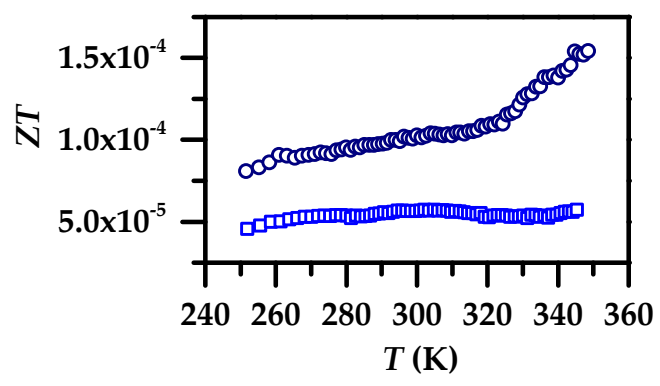

FIGURE 3.20: (a) Electrical conductivity, (b) thermopower, (c) thermal conductivity, and (d) figure of merit of as-synthesized and hydrazine capped $\mathrm{Bi}_{2} \mathrm{Te}_{3}$ synthesized at $120^{\circ} \mathrm{C}$ via bismuth nanoparticles.

\subsubsection{Doped $\mathrm{Bi}_{2} \mathrm{Te}_{3}$ nanostructures}

In an attempt to further increase the electrical conductivity of our nanostructured $\mathrm{Bi}_{2} \mathrm{Te}_{3}$ the effect of $\mathrm{Cu}, \mathrm{Co}$, and $\mathrm{Ni}$ dopants were investigated. In order to investigate the properties of doped $\mathrm{Bi}_{2} \mathrm{Te}_{3}$, the previous method used to synthesise $\mathrm{Bi}_{2} \mathrm{Te}_{3}$ at $120{ }^{\circ} \mathrm{C}$ via bismuth nanoparticles was modified by substituting the bismuth precursor for a stoichiometric dopant precursor.

Synthesis of $\mathrm{Cu}$ doped $\mathrm{Bi}_{2} \mathrm{Te}_{3}$ nanoparticles. $0.2 \mathrm{mmol}$ of bismuth(III) acetate was substituted with copper(II) acetylacetonate. The reaction was then carried out as per the synthesis of $\mathrm{Bi}_{2} \mathrm{Te}_{3}$ at $120{ }^{\circ} \mathrm{C}$.

Synthesis of $\mathrm{Ni}$ doped $\mathrm{Bi}_{2} \mathrm{Te}_{3}$ nanoparticles. $0.2 \mathrm{mmol}$ of bismuth(III) acetate was substituted with nickel(II) acetate tetrahydrate. The reactions was then carried out in a similar manor to $\mathrm{Bi}_{2} \mathrm{Te}_{3}$ synthesized at $120{ }^{\circ} \mathrm{C}$, except 
the degassing step for the bismuth nanoparticle precursor was increased to 2 hours.

Synthesis of Co doped $\mathrm{Bi}_{2} \mathrm{Te}_{3}$ nanoparticles. $0.2 \mathrm{mmol}$ of bismuth(III) acetate was substituted with cobalt(II) acetate tetrahydrate. The reaction was then carried out as per the synthesis of $\mathrm{Bi}_{2} \mathrm{Te}_{3}$ at $120{ }^{\circ} \mathrm{C}$, except the degassing step for the bismuth nanoparticle precursor was increased to 2 hours.

Ligand exchange and nanocomposite pellets. All materials were treated with the hydrazine ligand exchange as per the $\mathrm{Bi}_{2} \mathrm{Te}_{3}$ sample synthesized at $120{ }^{\circ} \mathrm{C}$. The nanocomposite pellets were pressed using CIP with a $5 \mathrm{~mm}$ die at $250 \mathrm{MPa}$ for 5 minutes.

\subsubsection{Characterisation and thermoelectric properties}

(a)

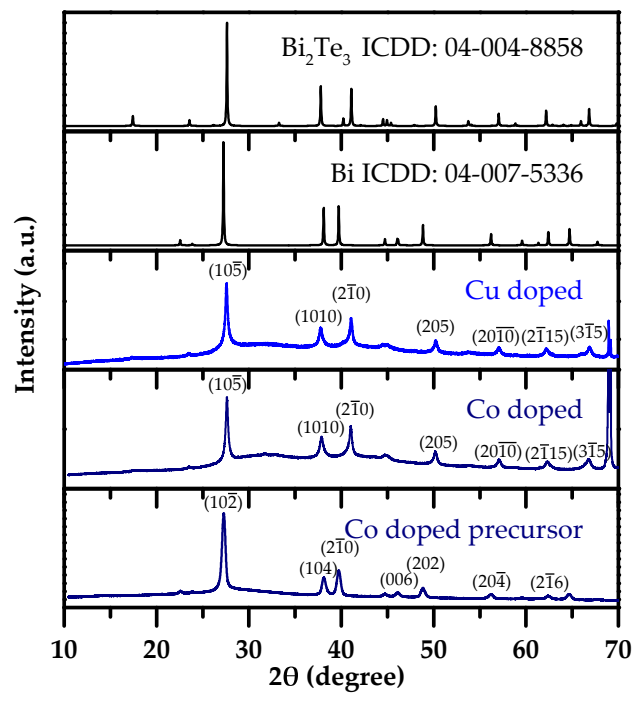

(b)

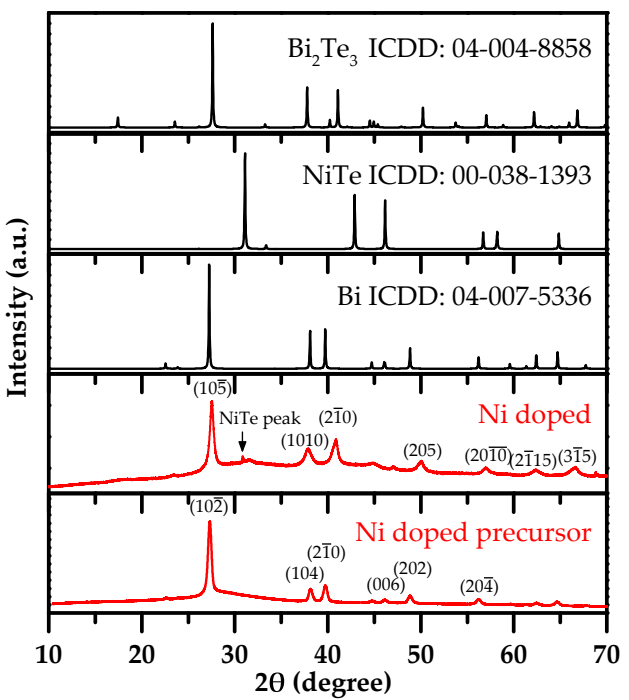

FIGURE 3.21: Powder XRD spectra of doped $\mathrm{Bi}_{2} \mathrm{Te}_{3}$ synthesised at $120^{\circ} \mathrm{C}$ and doped $\mathrm{Bi}$ nanoparticle precursor. (a) $\mathrm{Co}$ and $\mathrm{Cu}$ doped samples (b) Ni doped samples. Note the peak at $69^{\circ}$ is due to the Si substrate.

Powder XRD spectra were collected for $\mathrm{Cu}$, Co and $\mathrm{Ni}$ doped $\mathrm{Bi}_{2} \mathrm{Te}_{3}$ and the Bi nanoparticle precursors and are shown in Figure 3.21. The Co and Ni doped precursors appear to be crystalline and can be indexed to rhombohedral Bi (ICDD: 04-007-5336). The $\mathrm{Cu}$ and $\mathrm{Co}$ doped $\mathrm{Bi}_{2} \mathrm{Te}_{3}$ sample was indexed to rhombohedral $\mathrm{Bi}_{2} \mathrm{Te}_{3}$; no additional peaks corresponding to other phases were observed. The Ni doped sample was indexed to rhombohedral $\mathrm{Bi}_{2} \mathrm{Te}_{3}$, however an additional (101) peak was also observed that could be due to the presence of a NiTe phase. Using Scherrer's equation the crystallite size was estimated to be 18,23 , and $16 \mathrm{~nm}$ for $\mathrm{Cu}, \mathrm{Co}$, and $\mathrm{Ni}$ doped $\mathrm{Bi}_{2} \mathrm{Te}_{3}$ respectively. 
(a)

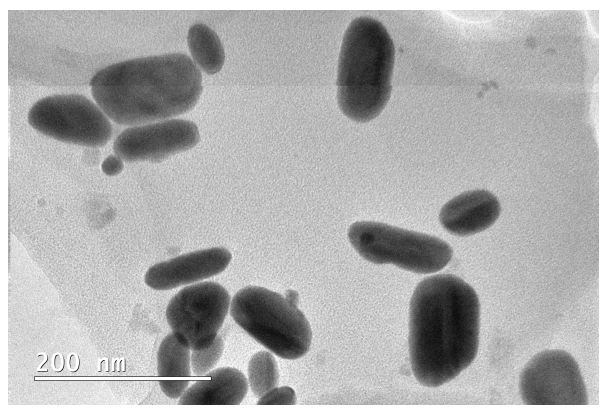

(b)

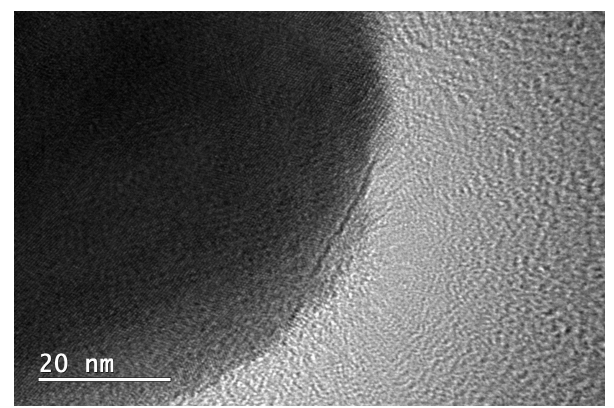

(c)

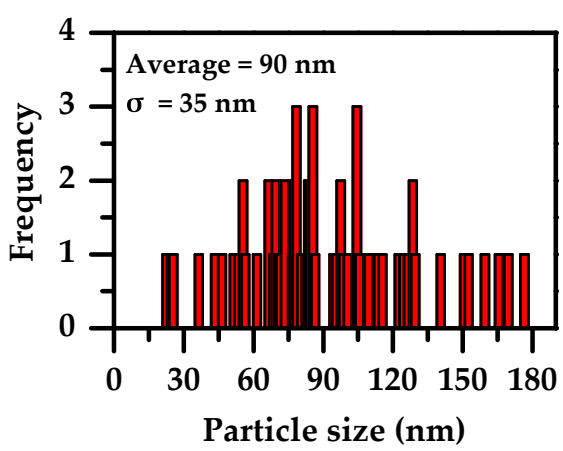

FIGURE 3.22: (a)(b) TEM micrographs and (c) particle distribution of $\mathrm{Cu}$ doped bismuth nanoparticle precursor.

TEM micrographs and particle distribution for $\mathrm{Cu}$ doped Bi nanoparticle precursor are shown in Figure 3.29. The TEM images indicate that the addition of copper(II) acetylacetonate has resulted in the size distribution and shape of the nanoparticles changing significantly relative to the undoped Bi nanoparticle precursor. The particles now appear to be more elliptical/rod shaped instead of the almost spherical like particles seen in Figure 3.12. The particle distribution is also extremely wide with an average diameter of $90 \mathrm{~nm}$ which is larger than the $41 \mathrm{~nm}$ particles synthesised without copper acetylacetonate present. It is unknown whether the presence of $\mathrm{Cu}$ or the acetylacetonate salt has resulted in the changes to the Bi nanoparticle precursor.

TEM micrographs and particle distribution of $\mathrm{Cu}$ doped $\mathrm{Bi}_{2} \mathrm{Te}_{3}$ are shown in Figure 3.23. The particles typically appear to be aggregated and consist of sub-15 nm nanoparticles. The size distribution of the particles is significantly more narrow and smaller than the $\mathrm{Cu}$ doped Bi nanoparticle precursor, although only particles with a well-defined shape were counted which may affect the overall distribution as extremely aggregated particles are not counted. The average particle size is only $11 \mathrm{~nm}$ which is smaller then the $36 \mathrm{~nm}$ of the undoped $\mathrm{Bi}_{2} \mathrm{Te}_{3}$ sample prepared at the same temperature. The nanoparticles appear to have similar shape to those reported by Scheele et al. for undoped $\mathrm{Bi}_{2} \mathrm{Te}_{3}$. The presence of copper acetylacetonate appears to have significantly altered the size and shape distribution of our $\mathrm{Bi}_{2} \mathrm{Te}_{3}$ nanoparticles allowing 
(a)

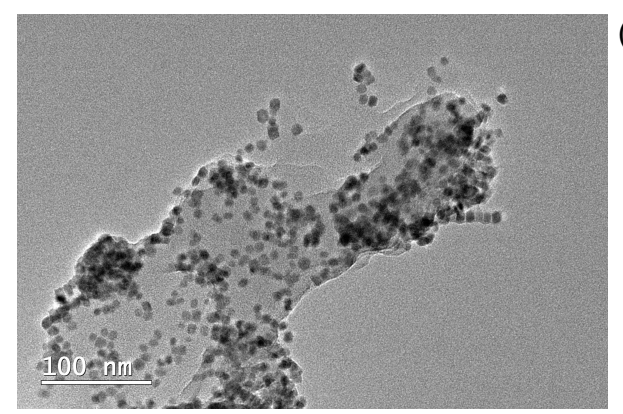

(c)

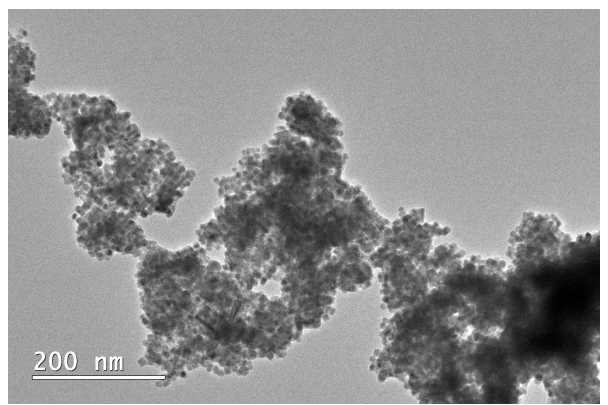

(b)

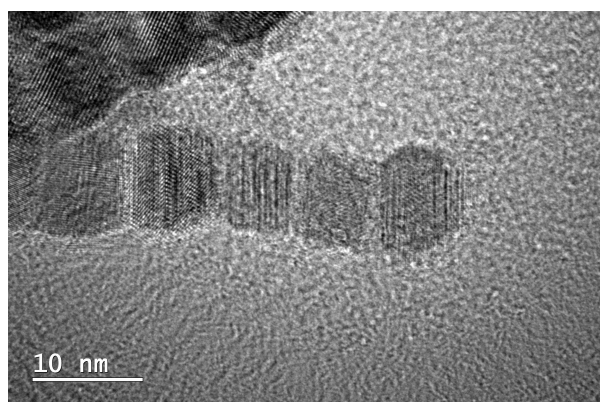

(d)

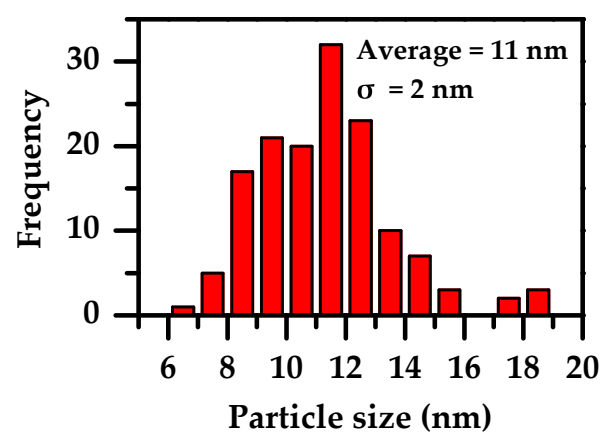

FIGURE 3.23: (a)(b)(c) TEM micrographs and (d) particle distribution of $\mathrm{Cu}$ doped $\mathrm{Bi}_{2} \mathrm{Te}_{3}$ synthesised at $120{ }^{\circ} \mathrm{C}$ via bismuth nanoparticles.

(a)

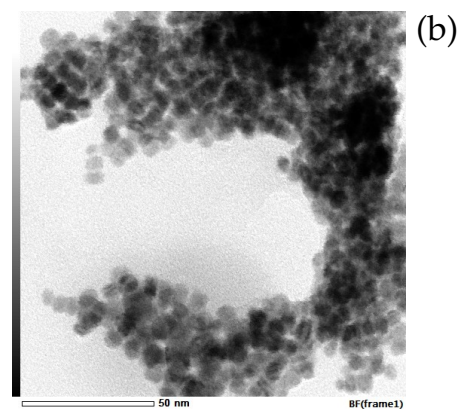

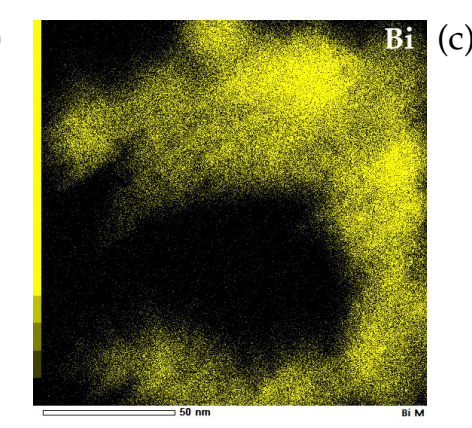

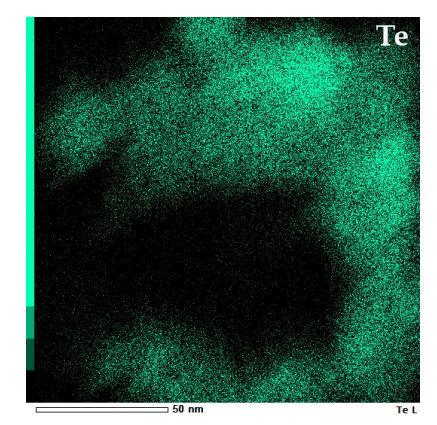

FIGURE 3.24: STEM/EDS elemental map of $\mathrm{Cu}$ doped $\mathrm{Bi}_{2} \mathrm{Te}_{3}$ synthesised at $120{ }^{\circ} \mathrm{C}$ via bismuth nanoparticles. (a) STEM-BF image (b) Bi map (c) Te map, the scale bar is $50 \mathrm{~nm}$.

us to achieve monodisperse sub-50 nm nanoparticles. The STEM/EDS and SEM/EDS maps for Co doped $\mathrm{Bi}_{2} \mathrm{Te}_{3}$ and the resulting nanocomposite pellet are shown in Figure 3.24 and Figure 3.25 respectively. The elemental map of $\mathrm{Cu}, \mathrm{Bi}$ and Te appear to be uniformly distributed across the pellet. The SEM/EDS elemental composition are shown in Table 3.7. We can see that we have an excess of Bi present which is to be expected for materials synthesized via Bi nanoparticle precursor ${ }^{40}$. The elemental composition shows us that we have successfully incorporated $2 \%$ of $\mathrm{Cu}$ relative to $\mathrm{Bi}$ into our $\mathrm{Bi}_{2} \mathrm{Te}_{3}$ nanoparticles. The percentage of $\mathrm{Cu}$ incorporated is less than the $10 \%$ by mole of $\mathrm{Cu}$ added to the $\mathrm{Bi}$ nanoparticle precursor. 
(a)

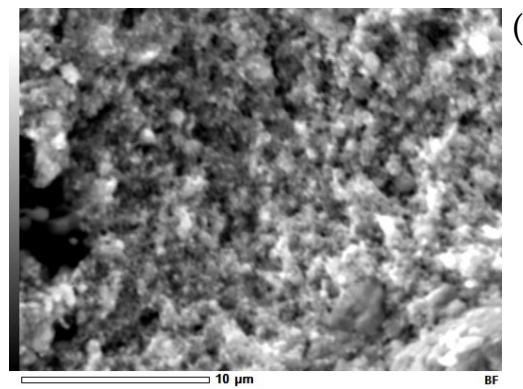

(b)

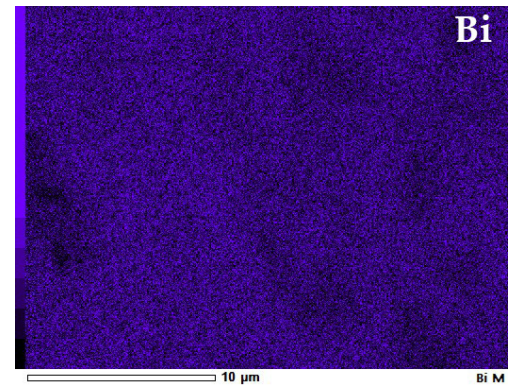

(c)

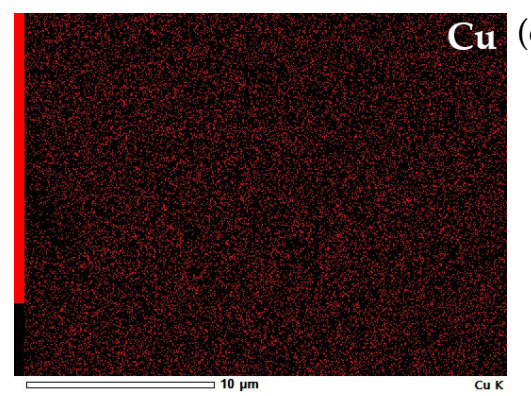

(d)

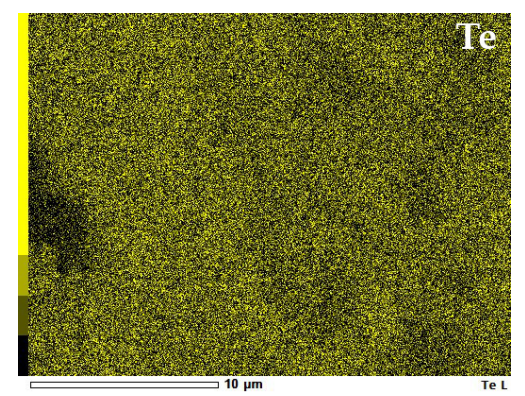

(e)

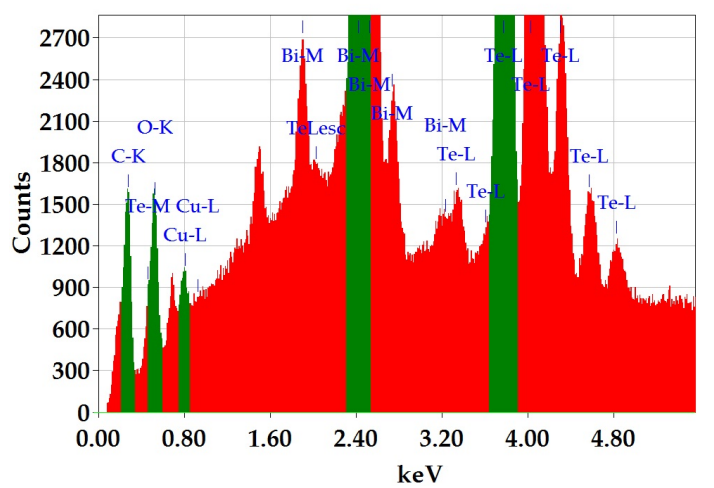

FIGURE 3.25: SEM/EDS elemental map of nanocomposite pellet prepared from $\mathrm{Cu}$ doped $\mathrm{Bi}_{2} \mathrm{Te}_{3}$ synthesised at $120^{\circ} \mathrm{C}$. (a) SEMSEI image (b) Bi map (c) Cu map (d) Te map, the scale bar is 10 $\mu \mathrm{m}$. (e) EDS spectrum showing presence of $\mathrm{Cu}$ peak.

TABLE 3.7: Composition determined by SEM/EDS for nanocomposite pellet of $\mathrm{Cu}$ doped $\mathrm{Bi}_{2} \mathrm{Te}_{3}$ synthesised at $120^{\circ} \mathrm{C}$ via bismuth nanoparticles.

\begin{tabular}{cccc}
\hline \multirow{2}{*}{ Reference image } & \multicolumn{4}{c}{ Normalized relative elemental composition } \\
\cline { 2 - 4 } & $\mathbf{B i}$ & $\mathbf{T e}$ & $\mathbf{C u}$ \\
\hline 1b-x5k & 1.97 & 2.59 & 0.03 \\
1b-x5k & 1.96 & 2.50 & 0.04 \\
1c-x5k & 1.96 & 2.38 & 0.04 \\
1d-x5k & 1.97 & 2.50 & 0.03 \\
1e-x5k & 1.95 & 2.57 & 0.05 \\
\hline Average & $\mathbf{1 . 9 6}$ & $\mathbf{2 . 5 9}$ & $\mathbf{0 . 0 4}$ \\
\hline$\sigma$ & $\mathbf{0 . 0 1}$ & $\mathbf{0 . 0 3}$ & $\mathbf{0 . 0 1}$ \\
\hline
\end{tabular}

The relative elemental composition is normalized with respect to $(\mathrm{Bi}+\mathrm{Cu})=2$. 
(a)

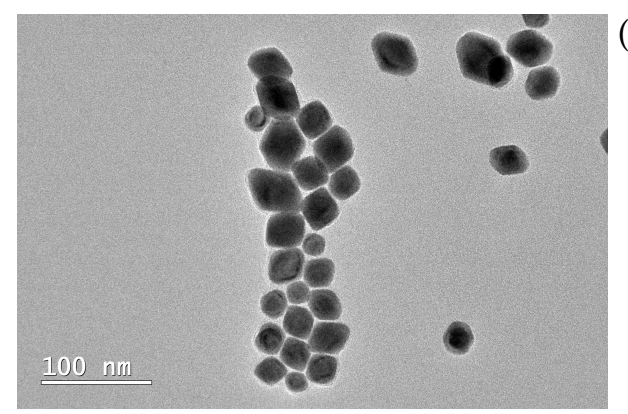

(b)

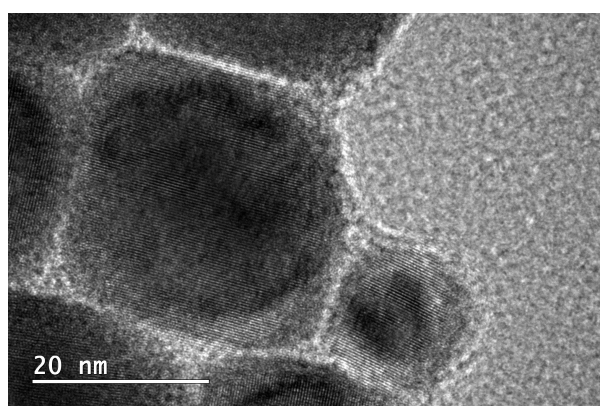

(c)

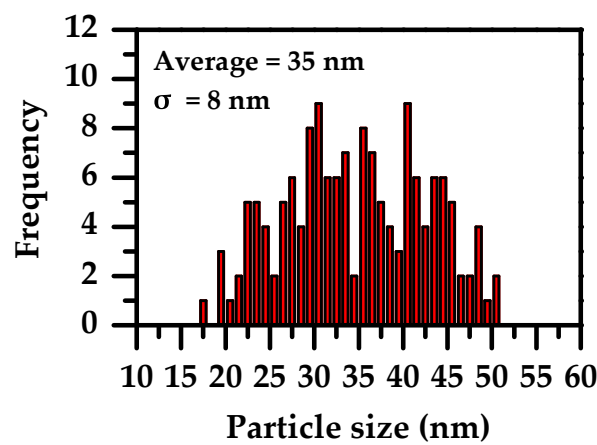

FIGURE 3.26: (a)(b) TEM micrographs and (c) particle distribution of Ni doped bismuth nanoparticle precursor.

TEM micrographs of $\mathrm{Ni}$ doped Bi nanoparticle precursor are shown in Figure 3.26. The addition of nickel(II) acetate tetrahydrate does not appear to have significantly changed the size or the particle distribution of the Bi nanoparticle precursor. The particles are still roughly spherical and have an average size of $35 \mathrm{~nm}$. TEM/EDS analysis indicates that no $\mathrm{Ni}$ is present in the Bi nanoparticle precursor sample. TEM micrographs of $\mathrm{Ni}$ doped $\mathrm{Bi}_{2} \mathrm{Te}_{3}$ are shown in Figure 3.27. The particles appear to be extremely aggregated. Particles with a well-defined shape appear to be rhomboid and sub-100 nm. The morphology of the $\mathrm{Ni}$ doped $\mathrm{Bi}_{2} \mathrm{Te}_{3}$ appears to be similar to the undoped sample synthesized at the same temperature. The particle distribution can not be determined due to the highly aggregated nature of the nanoparticles, however the particles with a well defined shape appear to be sub-100 nm. SEM/EDS analysis of nanocomposite pellets of $\mathrm{Ni}$ doped $\mathrm{Bi}_{2} \mathrm{Te}_{3}$ are shown in Figure 3.28. The elemental map indicates that $\mathrm{Bi}, \mathrm{Ni}$ and $\mathrm{Te}$ are all uniformly distributed. The elemental composition determined from EDS of the Ni doped $\mathrm{Bi}_{2} \mathrm{Te}_{3}$ pellet is shown in Table 3.8. We can see that we have similar $(\mathrm{Bi}+\mathrm{Ni})$ rich material. The amount of Te present is similar to the undoped $\mathrm{Bi}_{2} \mathrm{Te}_{3}$ pellet. The elemental composition indicates we have successfully incorporated $10.5 \%$ of $\mathrm{Ni}$ relative to $\mathrm{Bi}$. The incorporation of $\mathrm{Ni}$ appears to be exactly the same as the stoichiometric addition of the precursor, showing no preferential inclusion of $\mathrm{Ni}$ relative to $\mathrm{Bi}$. The percentage of $\mathrm{Ni}$ present also suggests that we have 
(a)

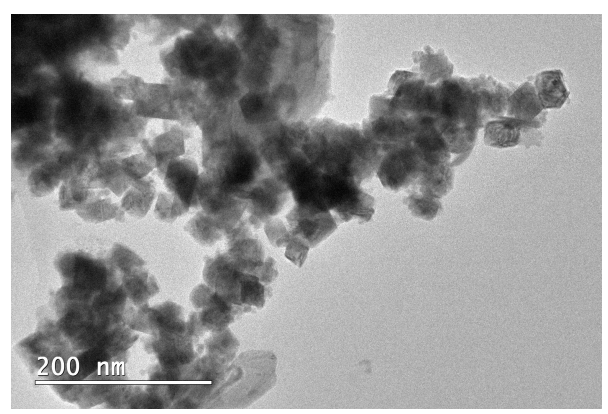

(c)

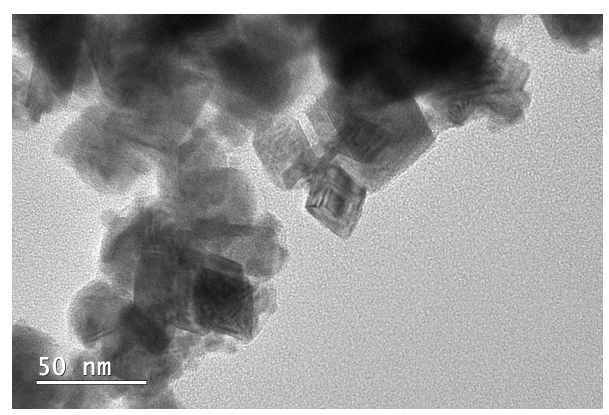

(b)

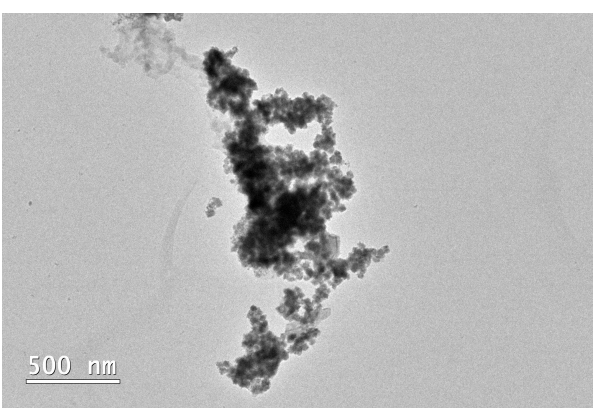

(d)

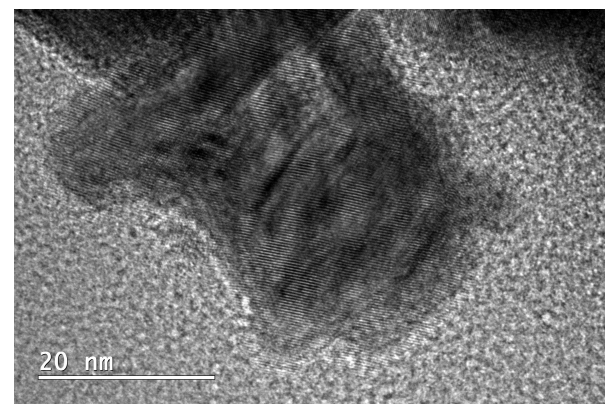

FIGURE 3.27: TEM micrographs of $\mathrm{Ni}$ doped $\mathrm{Bi}_{2} \mathrm{Te}_{3}$ synthesised at $120^{\circ} \mathrm{C}$ via bismuth nanoparticles.

(a)

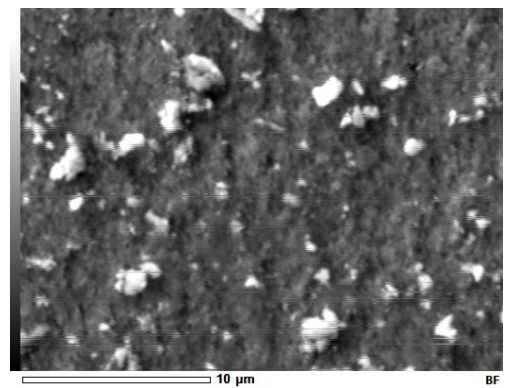

(c)

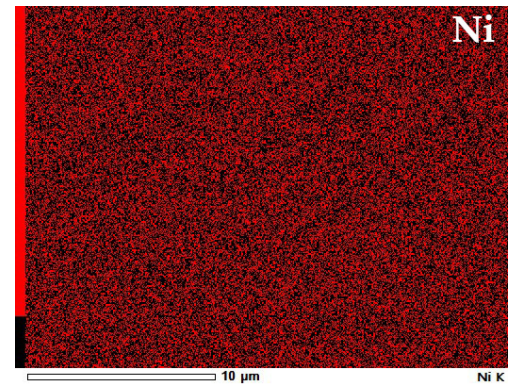

(b)

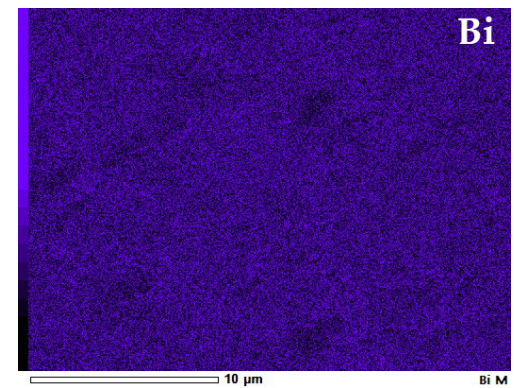

(d)

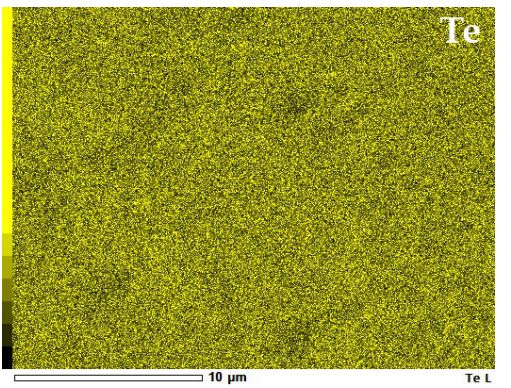

FIGURE 3.28: SEM/EDS elemental map of nanocomposite pellet prepared from $\mathrm{Ni}$ doped $\mathrm{Bi}_{2} \mathrm{Te}_{3}$ synthesised at $120{ }^{\circ} \mathrm{C}$ via bismuth nanoparticles spin cast on $\mathrm{SiO}_{2}$. (a) SEM-SEI image (b) Bi map (c) Ni map (d) Te map, the scale bar is $10 \mu \mathrm{m}$.

formed an alloy rather than a doped $\mathrm{Bi}_{2} \mathrm{Te}_{3}$ nanostructure, which is consistent with the observed NiTe peak present in the XRD spectra. 
TABLE 3.8: Composition determined by SEM/EDS for nanocomposite pellet of Ni doped $\mathrm{Bi}_{2} \mathrm{Te}_{3}$ synthesised at $120^{\circ} \mathrm{C}$ via bismuth nanoparticles.

\begin{tabular}{cccc}
\hline \multirow{2}{*}{ Reference image } & \multicolumn{3}{c}{ Normalized relative elemental composition } \\
\cline { 2 - 4 } & $\mathbf{B i}$ & $\mathbf{T e}$ & $\mathbf{N i}$ \\
\hline 1a-x5k & 1.80 & 2.61 & 0.20 \\
1b-x5k & 1.81 & 2.60 & 0.19 \\
1c-x5k & 1.80 & 2.64 & 0.20 \\
1d-x5k & 1.80 & 2.62 & 0.20 \\
1e-x5k & 1.80 & 2.60 & 0.20 \\
\hline Average & $\mathbf{1 . 8 1}$ & $\mathbf{2 . 5 9}$ & $\mathbf{0 . 2 1}$ \\
\hline$\sigma$ & $\mathbf{0 . 0 0 3}$ & $\mathbf{0 . 0 1}$ & $\mathbf{0 . 0 0 3}$ \\
\hline
\end{tabular}

The relative elemental composition is normalized with respect to $(\mathrm{Bi}+\mathrm{Ni})=2$.

TEM micrographs and particle distribution of Co doped Bi nanoparticles are shown in Figure 3.29. The particles appear to be roughly spherical with a narrow size distribution and an average size of $27 \mathrm{~nm}$. The average size of the particles is slightly smaller than the $41 \mathrm{~nm}$ for the undoped Bi nanoparticle precursor. TEM/EDS analysis of the Co doped Bi nanoparticle precursor is shown in Table 3.9. The average $\mathrm{Co} / \mathrm{Bi}$ ratio was found to be 0.08 which is similar to the nominal precursor ratio of 0.10. TEM micrographs of $\mathrm{Co}$

(a)

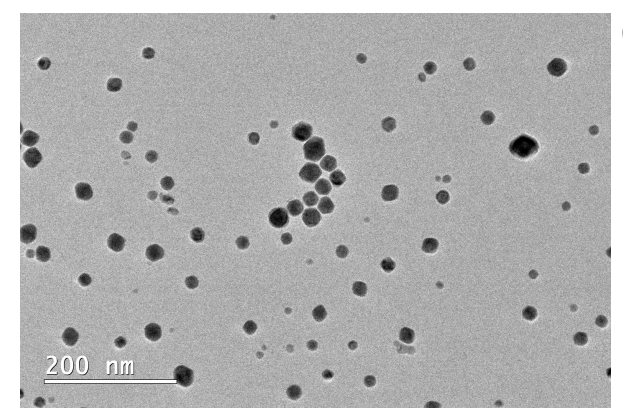

(b)

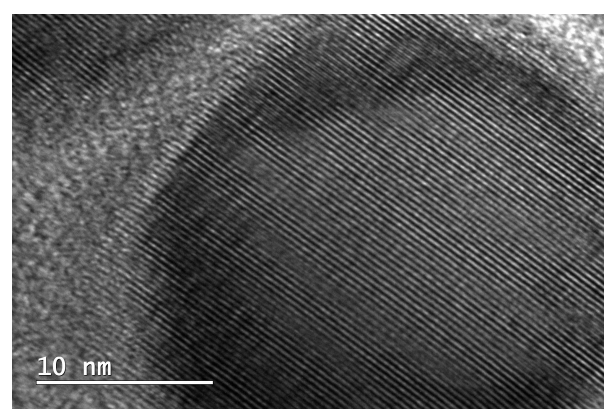

(c)

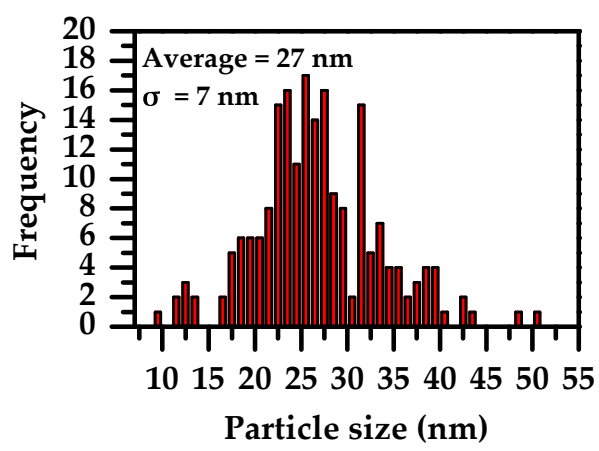

FIGURE 3.29: (a)(b) TEM micrographs and (c) particle distribution of Co doped bismuth nanoparticle precursor. 
TABLE 3.9: Composition determined by TEM/EDS for Co doped bismuth nanoparticle precursor.

\begin{tabular}{cc}
\hline Reference Image & Co/Bi Ratio \\
\hline 1a-x10k & 0.12 \\
1b-x30k & 0.08 \\
1c-x30k & 0.07 \\
1d-x30k & 0.03 \\
1a-x800k-STEM & 0.13 \\
\hline Average & $\mathbf{0 . 0 8}$ \\
\hline$\sigma$ & $\mathbf{0 . 0 4}$ \\
\hline
\end{tabular}

(a)

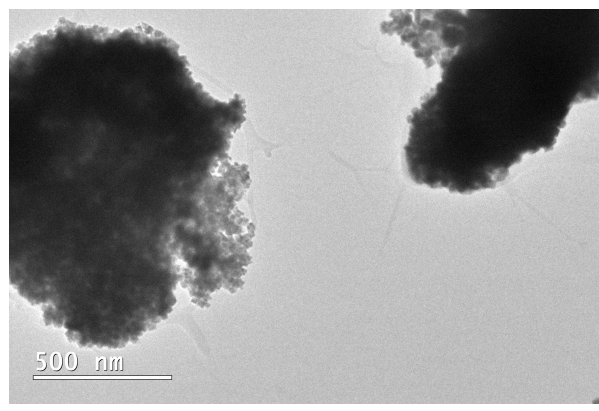

(c)

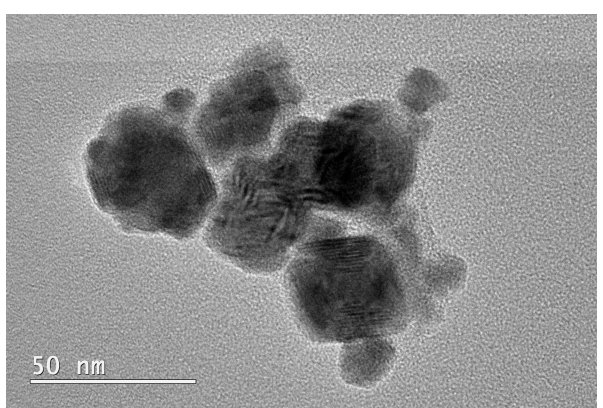

(b)

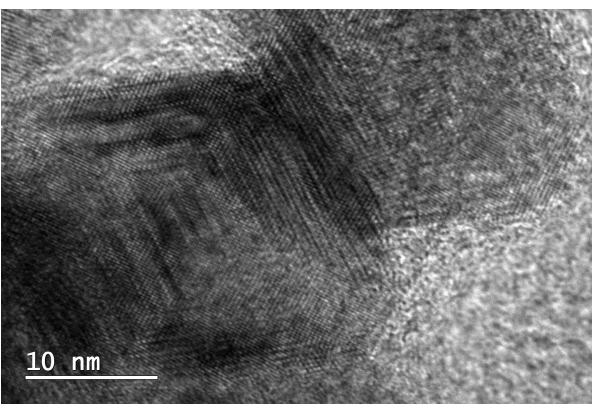

(d)

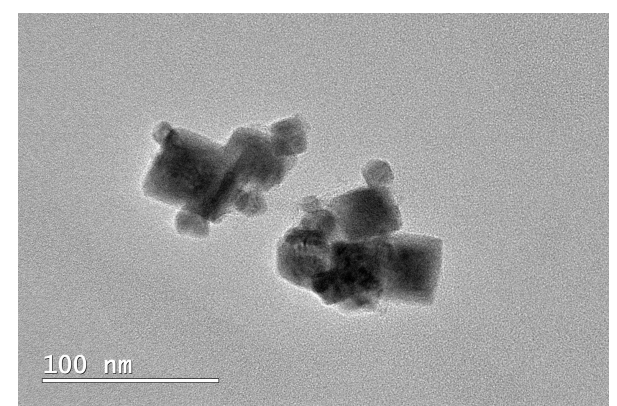

FIGURE 3.30: TEM micrographs of Co doped $\mathrm{Bi}_{2} \mathrm{Te}_{3}$ synthesised at $120^{\circ} \mathrm{C}$ via bismuth nanoparticles.

doped $\mathrm{Bi}_{2} \mathrm{Te}_{3}$ are shown in Figure 3.30. The particles seem to be extremely aggregated, even more so than the undoped $\mathrm{Bi}_{2} \mathrm{Te}_{3}$ nanoparticles. This is most likely due to the poor colloidal stability of the nanoparticles which tended to form a gel in toluene and was only partially dispersed in chloroform with typical colloidal stability $<5$ minutes. The aggregated particles appear to have a rhomboid shape that is sub-100 nm which is similar to the undoped $\mathrm{Bi}_{2} \mathrm{Te}_{3}$ nanostructures. SEM/EDS analysis was carried out on a nanocomposite pellet of Co doped $\mathrm{Bi}_{2} \mathrm{Te}_{3}$ and are shown in Figure 3.31. The SEM-SEI image clearly indicates a significant amount of interfacing present for compacted nanoparticles. The elemental map shows a uniform distribution or $\mathrm{Bi}, \mathrm{Co}$ and Te. The elemental composition determined from EDS maps at several different 
(a)

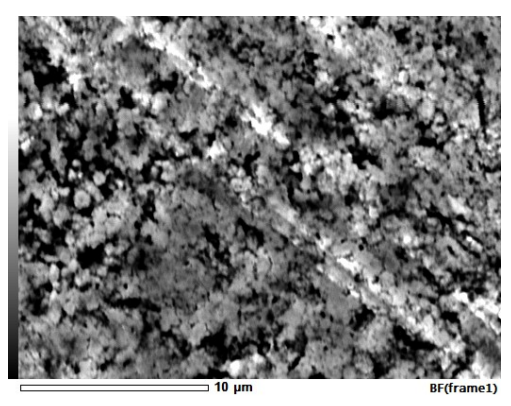

(c)

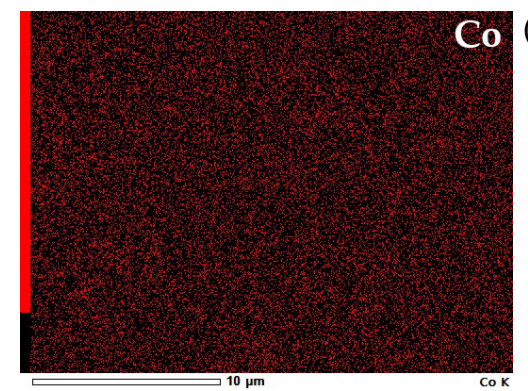

(b)

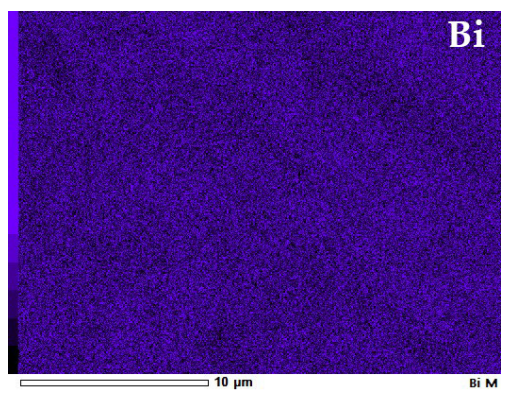

(d)

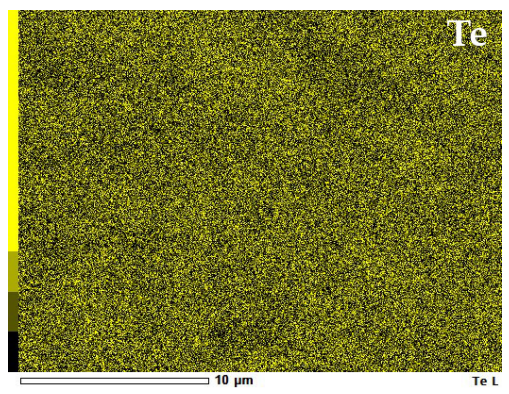

FIGURE 3.31: SEM/EDS elemental map of nanocomposite pellet prepared from Co doped $\mathrm{Bi}_{2} \mathrm{Te}_{3}$ synthesised at $120^{\circ} \mathrm{C}$ via bismuth nanoparticles. (a) SEM-SEI image (b) Bi map (c) Co map (d) Te map, the scale bar is $10 \mu \mathrm{m}$.

TABLE 3.10: Composition determined by SEM/EDS for nanocomposite pellet of Co doped $\mathrm{Bi}_{2} \mathrm{Te}_{3}$ synthesised at $120^{\circ} \mathrm{C}$ via bismuth nanoparticles.

\begin{tabular}{cccc}
\hline \multirow{2}{*}{ Reference image } & \multicolumn{4}{c}{ Normalized relative elemental composition } \\
\cline { 2 - 4 } & $\mathbf{B i}$ & Te & Co \\
\hline 1a-x5k & 1.74 & 2.83 & 0.26 \\
1b-x5k & 1.58 & 2.84 & 0.42 \\
1c-x5k & 1.76 & 2.83 & 0.24 \\
1d-x5k & 1.75 & 2.83 & 0.25 \\
1e-x5k & 1.74 & 2.83 & 0.26 \\
\hline Average & $\mathbf{1 . 7 1}$ & $\mathbf{2 . 8 3}$ & $\mathbf{0 . 2 9}$ \\
\hline$\sigma$ & $\mathbf{0 . 0 7}$ & $\mathbf{0 . 0 0 3}$ & $\mathbf{0 . 0 7}$
\end{tabular}

The relative elemental composition is normalized with respect to $(\mathrm{Bi}+\mathrm{Co})=2$.

positions is summarized in Table 3.10. The average composition shows an excess of $(\mathrm{Bi}+\mathrm{Co})$ like previous reactions however this sample appears to have a higher Te content than the undoped $\mathrm{Bi}_{2} \mathrm{Te}_{3}$ sample. The average Co inclusion is $14.5 \%$ relative to $\mathrm{Bi}$ which is higher than the $10 \%$ initial precursor concentration. This suggests that Co is preferential incorporated into $\mathrm{Bi}_{2} \mathrm{Te}_{3}$ relative to $\mathrm{Bi}$. This preferential incorporation of Co may also explain the higher percentage of Te present relative to undoped $\mathrm{Bi}_{2} \mathrm{Te}_{3}$. The amount of $\mathrm{Co}$ present also suggests that we have formed an alloy rather than doped $\mathrm{Bi}_{2} \mathrm{Te}_{3}$ 
(a)

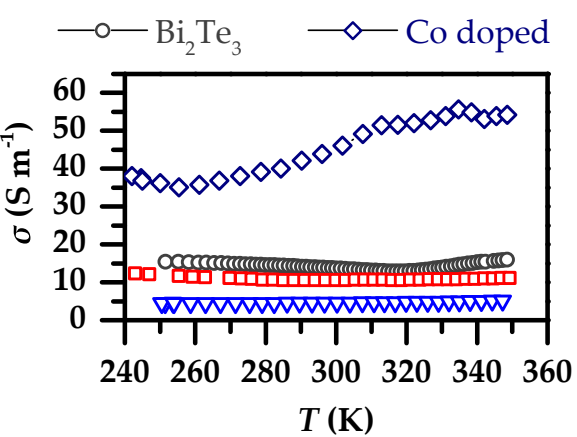

(c)

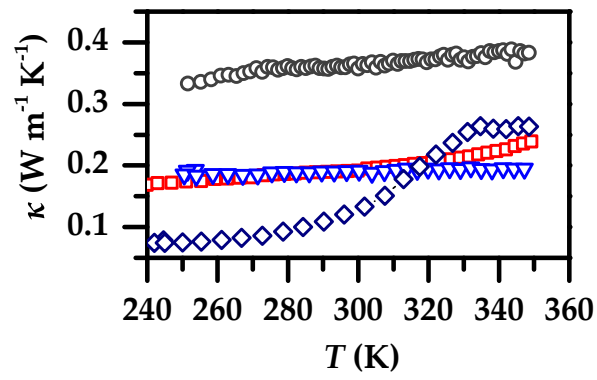

(b)

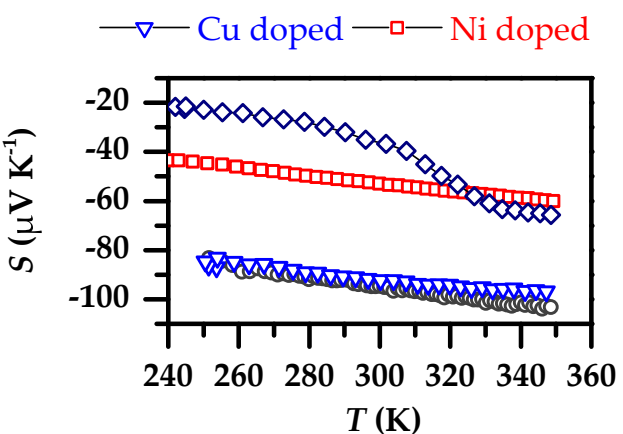

(d)

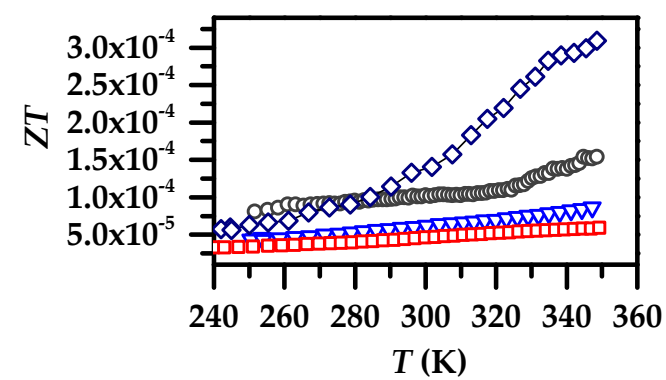

FIGURE 3.32: (a) Electrical conductivity, (b) thermopower, (c) thermal conductivity, and (d) figure of merit of $\mathrm{Cu}, \mathrm{Ni}$, and $\mathrm{Co}$ doped/alloyed $\mathrm{Bi}_{2} \mathrm{Te}_{3}$ nanoparticles.

structure.

The thermoelectric properties of $\mathrm{Cu}, \mathrm{Co}$, and $\mathrm{Ni}$ doped $\mathrm{Bi}_{2} \mathrm{Te}_{3}$ nanostructures are shown in Figure 3.32 and a comparison of the thermoelectric properties at room temperature is summarized in Table 3.11. The electrical conductivity of the $\mathrm{Cu}$ and $\mathrm{Ni}$ doped samples is less than the undoped $\mathrm{Bi}_{2} \mathrm{Te}_{3}$ sample. The decrease in the $\mathrm{Cu}$ doped $\mathrm{Bi}_{2} \mathrm{Te}_{3}$ electrical conductivity may be an artefact of the size and shape of the nanoparticles, as the $\mathrm{Cu}$ doped particles are much smaller and will thus have more interfacing when compacted together. The interfacing can act as barrier for charge transport between the particles. The electrical conductivity of the Ni doped sample does not appear to have changed significantly. The Co doped electrical conductivity appears to have increased by 4 times at room temperature relative to the undoped $\mathrm{Bi}_{2} \mathrm{Te}_{3}$ sample. The thermopower of $\mathrm{Cu}$ doped and undoped $\mathrm{Bi}_{2} \mathrm{Te}_{3}$ are similar and comparable with that reported by Scheele et al. (2009) for $\mathrm{Bi}_{2} \mathrm{Te}_{3}$ nanoparticles. The thermopower of the $\mathrm{Ni}$ and Co doped sample appears to be significantly less than the undoped $\mathrm{Bi}_{2} \mathrm{Te}_{3}$. This decrease maybe due to the presence of a new phase in the $\mathrm{Ni}$ and Co doped samples as the percentage of dopant present is quite high, suggesting the formation of an alloy. The thermal conductivity of all the doped $\mathrm{Bi}_{2} \mathrm{Te}_{3}$ samples is less than the undoped $\mathrm{Bi}_{2} \mathrm{Te}_{3}$. In the case of the $\mathrm{Cu}$ doped sample the decrease in the thermal conductivity is most likely due to increased interfacing arising from the much smaller size 
TABLE 3.11: Comparison of thermoelectric properties of nanostructured and bulk $\mathrm{Bi}_{2} \mathrm{Te}_{3}$ samples at $300 \mathrm{~K}$.

\begin{tabular}{lccccc}
\hline Material & $\boldsymbol{\sigma}\left(\mathrm{Sm}^{-1}\right)$ & $\boldsymbol{S}(\mu \mathrm{V} \mathrm{K}$ \\
\hline bulk $\mathrm{p}-\mathrm{Bi}_{2} \mathrm{Te}_{3}$ & 108000 & 173 & $\boldsymbol{\kappa}\left(\mathrm{W} \mathrm{m}^{-1} \mathrm{~K}^{-1}\right)$ & $\boldsymbol{Z T}$ & REF \\
bulk $\mathrm{n}-\mathrm{Bi}_{2} \mathrm{Te}_{3}$ & 42000 & -209 & 0.8 & 1.00 & 2 \\
$\mathrm{n}-\mathrm{Bi}_{2} \mathrm{Te}_{3}$ & 130000 & -185 & 1.23 & 0.68 & 2 \\
$\mathrm{n}-\mathrm{Bi}_{2} \mathrm{Te}_{3}$ & 118000 & -140 & 0.8 & 0.87 & 33 \\
$\mathrm{n}-\mathrm{Bi}_{2} \mathrm{Te}_{3}$ & 77000 & -80 & 0.8 & 0.18 & 40 \\
$\mathrm{n}-\mathrm{Bi}_{2} \mathrm{Te}_{3}$ & 90000 & -170 & 2 & 0.39 & 38 \\
$\mathrm{Bi}_{2} \mathrm{Te}_{3}$ & 13.1 & -99 & 0.38 & $1.0 \times 10^{-4}$ & this work \\
$\mathrm{Cu}$ doped $\mathrm{Bi}_{2} \mathrm{Te}_{3}$ & 4.6 & -92 & 0.19 & $6.2 \times 10^{-5}$ & this work \\
$\mathrm{Ni}$ doped $\mathrm{Bi}_{2} \mathrm{Te}_{3}$ & 11 & -53 & 0.19 & $4.7 \times 10^{-5}$ & this work \\
Co doped $\mathrm{Bi}_{2} \mathrm{Te}_{3}$ & 46 & -37 & 0.13 & $1.4 \times 10^{-4}$ & this work \\
\hline
\end{tabular}

of the nanoparticles. In the Co and Ni doped sample the decrease is most likely due to a decrease in the lattice contribution to the thermal conductivity. The $\mathrm{Co}^{2+}(\sim 79 \mathrm{pm})$ and $\mathrm{Ni}^{2+}(\sim 83 \mathrm{pm})$ radii are lower than that of $\mathrm{Bi}^{3+}(\sim 117$ pm) which can result in a lattice distortion in $\mathrm{Bi}_{2-\mathrm{x}} \mathrm{M}_{\mathrm{x}} \mathrm{Te}_{3}$ (where $\mathrm{M}$ is the dopant) crystal structure. This will result in a strong anharmonicty of bonding arrangement which could lead to phonon-phonon umklapp scattering ${ }^{76,77}$. Point defects could also enhance phonon scattering due to mass difference and size differences between guest atom and those of the host lattice ${ }^{76-78}$. These process have been shown to decrease the lattice thermal conductivity in doped nanostructured and doped single crystal materials ${ }^{76-78}$. Overall the figure of merit is worst after doping/alloying with $\mathrm{Cu}$ and Ni. This suggests that doping/alloying $\mathrm{Bi}_{2} \mathrm{Te}_{3}$ with these materials may not be a suitable approach. Co doped/alloyed $\mathrm{Bi}_{2} \mathrm{Te}_{3}$ has resulted in a $40 \%$ increase in the figure of merit at room temperature relative to undoped $\mathrm{Bi}_{2} \mathrm{Te}_{3}$, this shows the potential for Co to be used to further increase the figure of merit. Although initial experiments show $\mathrm{Ni}$ and $\mathrm{Cu}$ having minimal effect on the figure of merit, further experiments must be carried out to optimise the thermoelectric properties with respect to the dopant concentration. 


\subsection{Brief investigation of alternative nanoparticles}

\subsubsection{SnSe nanoparticles}

Other novel nanomaterials are also investigated for applications in thermoelectrics. SnSe single crystals have already be shown to be exceptional thermoelectric materials owing to their high power factors and exceptionally low thermal conductivities ${ }^{12,13,41}$. Currently experiments are still ongoing in order to synthesize enough material to produced pellets for thermoelectric measurements. Here we present a brief look at the synthesis and characterisation of SnSe nanoparticles. All reactions were carried out using standard Schlenk line techniques under vacuum/nitrogen.

\subsubsection{Synthesis}

SnSe was synthesized using a modified method reported by Liu et al. $(2014)^{79}$. Several synthesis attempts are listed below with various reaction conditions.

Synthesis of OAm-Se precursor. $4 \mathrm{mmol}$ of Se pellets $(0.3160 \mathrm{~g})$ was added to $20 \mathrm{~mL}$ of technical grade oleylamine (70\%). The solution was then degassed for $30 \mathrm{mins}$ at $120^{\circ} \mathrm{C}$. The solution was then purged with nitrogen and heated to $300-320^{\circ} \mathrm{C}$. During the heating the solution was observed to transition to a brown/orange colour and then to dark brown/black. After 2 hours the solution was cooled down to $100{ }^{\circ} \mathrm{C}$ and was used immediately to synthesise SnSe.

Synthesis of OA-Se precursor. $2 \mathrm{mmol}$ of Se powder $(0.1579 \mathrm{~g})$ was added to $20 \mathrm{~mL}$ of technical grade oleic acid( $90 \%)$ in a round bottom flask. The solution was degassed and heated to $120^{\circ} \mathrm{C}$. After $30 \mathrm{mins}$ the solution was purged with nitrogen and heated to $310-330{ }^{\circ} \mathrm{C}$. During the heating the black Se powder was observed to dissolve at $\sim 210{ }^{\circ} \mathrm{C}$ to form a yellow solution. After one and half hours the solution was cooled down to room temperature naturally upon which a slightly yellow gel forms. The OA-Se precursor was then stored under nitrogen and the gel is melted with a heat gun before being used to synthesis SnSe.

Synthesis of SnSe nanoparticles (TS1). $4 \mathrm{mmol}$ of $\mathrm{SnCl}_{2}(0.7629 \mathrm{~g})$ was added to $40 \mathrm{~mL}$ of 1-octadecene(ODE, 90\%), $16 \mathrm{~mL}$ of oleylamine(OLA,70\%), and $4 \mathrm{~mL}$ of oleic acid(OA, 90\%) in a round bottom flask. The solution was then degassed and slowly heated to $120^{\circ} \mathrm{C}\left(\sim 30 \mathrm{mins}\right.$ to reach $\left.120{ }^{\circ} \mathrm{C}\right)$. After 30 mins at $120^{\circ} \mathrm{C}$ the solution was purged with nitrogen and heated to 150 ${ }^{\circ} \mathrm{C}$. The solution was observed to have slightly yellow colour after degassing. 
$20 \mathrm{~mL}$ of the OAm-Se precursor was then injected into the Sn precursor solution, upon which the solution transitioned to a dark brown/black colour rapidly. The reaction was held at $145^{\circ} \mathrm{C}$ for 1 hour under nitrogen and then cooled down to room temperature. During the cooling $10 \mathrm{~mL}$ of toluene was added to prevent the formation of a gel. Ethanol (antisolvent) was then added to precipitate the nanoparticles. The nanoparticles were then collected using centrifugation at 6000 RPM for 10 mins. The nanoparticles were then redispersed in toluene (solvent) and the washing step was repeated 2 more times. The nanoparticles were then dried under vacuum and stored in a vacuum desicator.

Synthesis of SnSe nanoparticles (TS2). $1 \mathrm{mmol}$ of $\mathrm{SnCl}_{2}(0.1896 \mathrm{~g})$ was added to $20 \mathrm{~mL}$ of ODE, $8 \mathrm{~mL}$ of OAm, and $2 \mathrm{~mL}$ of OA. The solution was then degassed and slowly heated to $120^{\circ} \mathrm{C}$. After $30 \mathrm{mins}$ at $120^{\circ} \mathrm{C}$ the solution was observed to have a slightly yellow tinge to it. The solution was then purged with nitrogen and the reaction was heated to $155^{\circ} \mathrm{C}$. The reaction was held at this temperature for 15 minutes while the OA-Se precursor was heated to 80 ${ }^{\circ} \mathrm{C}$. It was observed after $10 \mathrm{mins}$ at $155^{\circ} \mathrm{C}$ that the $\mathrm{Sn}$ precursor solution had formed a faint brown ring just above the liquid level in the round bottom flask. $10 \mathrm{~mL}$ of OA-Se precursor was then injected into the $\mathrm{Sn}$ precursor and the reaction was held at $150{ }^{\circ} \mathrm{C}$ for 5 minutes, upon which the solution turned a dark brown/black colour. The solution was then cooled to room temperature naturally. Ethanol (antisolvent) was then added and the nanoparticles were collected using centrifugation at 6000 RPM for 10 mins. The nanoparticles was then redispersed in toluene (solvent) and the washing step was repeated 2 more times.

Synthesis of SnSe nanoparticles (TS3). $2 \mathrm{mmol}$ of $\mathrm{SnCl}_{2}$ was added to $40 \mathrm{~mL}$ of ODE, $16 \mathrm{~mL}$ of OAm, and $4 \mathrm{~mL} \mathrm{OA}$. The solution was degassed and slowly heated to $115{ }^{\circ} \mathrm{C}$ in increments $\left(20-100^{\circ} \mathrm{C}\right.$ in 2 hours, $100-115$ ${ }^{\circ} \mathrm{C}$ in 1 hour). The solution was then purged with nitrogen and heated to $155{ }^{\circ} \mathrm{C}$. Simultaneously the OA-Se precursor was heated to $80{ }^{\circ} \mathrm{C}$ under nitrogen. $20 \mathrm{~mL}$ of the OA-Se precursor was then injected into the Sn precursor and held at $150{ }^{\circ} \mathrm{C}$. The solution was observed to form a dark red/brown solution initially and then after 30 seconds the solution was observed to turn into a black colour. After 5 minutes the solution was cooled down to room temperature using a water bath. During the cooling $10 \mathrm{~mL}$ of toluene was added. Ethanol (antisolvent) was then added an the nanoparticles were collected using centrifugation at 6000 RPM for 10 mins. The nanoparticles were then redispersed in toluene (solvent) and the washing step was repeated 
2 more times.

\subsubsection{Results}

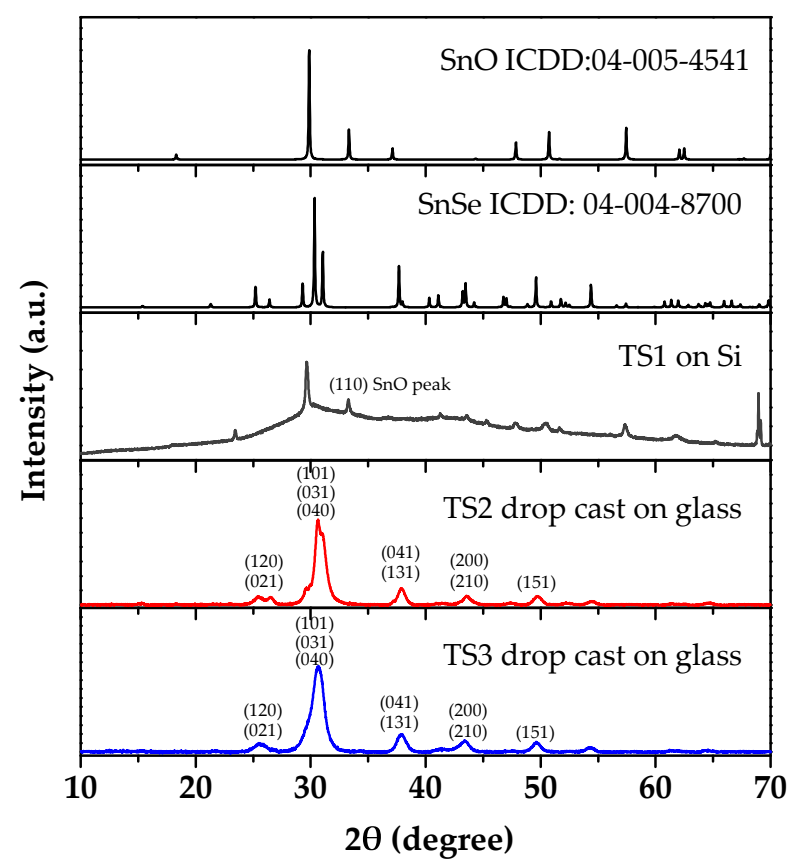

FIGURE 3.33: Powder XRD spectra of SnSe nanoparticles.

The powder XRD spectra for our initial SnSe nanoparticle synthesis attempts are shown in Figure 3.33. All three samples appear to be crystalline where TS2 and TS3 can be indexed to orthorhombic SnSe (ICDD: 04-004-8700). However TS1 appears to have peaks consistent with SnO suggesting that an oxide species has formed during this reaction.

In the reaction for TS1 a OAm-Se precursor was used. It was observed that during the heating of the OAm-Se precursor from $120-320^{\circ} \mathrm{C}$ that the solution initially turned orange/brown and then transitioned to a brown/black solution. The brown/black material indicates the formation of selenium oxide, which would account for the observed $\mathrm{SnO}$ phase present in the XRD. It was also found that using OA-Se precursor did not result in a $\mathrm{SnO}$ phase being formed as the Se precursor was not observed to oxidise in the presence of oleic acid.

TEM micrographs and elemental maps for TS1 are shown in Figure 3.34 and Figure 3.35. The nanoparticles appear to have a wide shape distribution and are typically sub-50 $\mathrm{nm}$ in size. The elemental maps indicate that we have a uniform distribution of $\mathrm{Sn}$ and Se. The elemental compositions determined from TEM/EDS are shown in Table 3.12. Our SnSe nanoparticles 
(a)

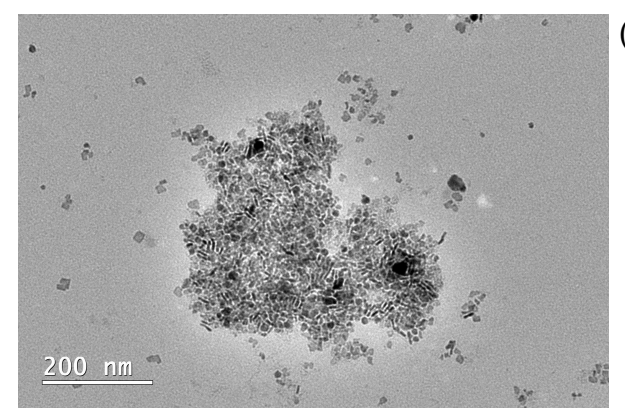

(b)

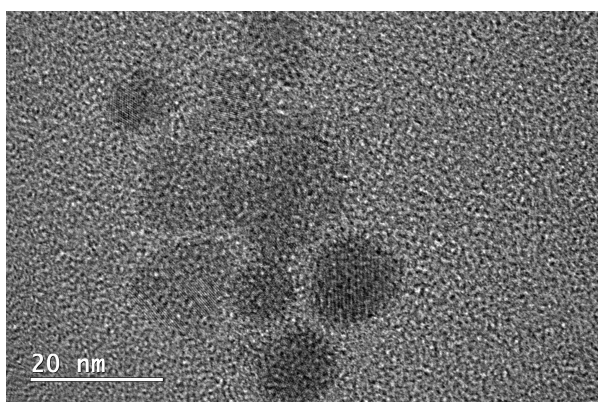

FIGURE 3.34: TEM micrographs of SnSe(TS1) nanoparticles.

(a)

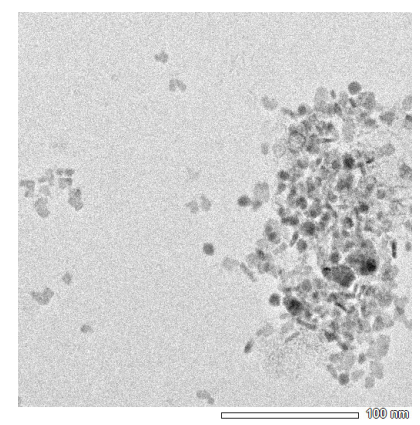

(b)

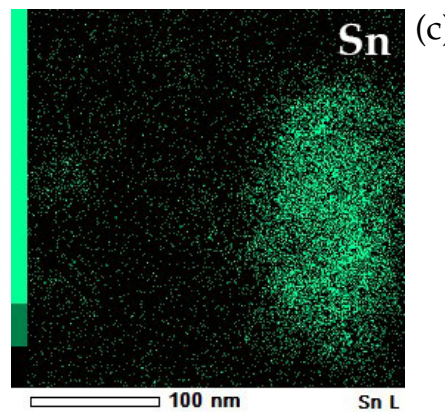

(c)

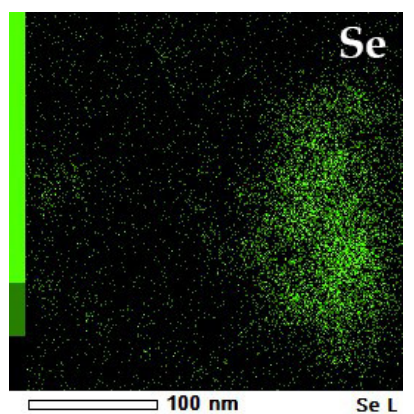

FIGURE 3.35: STEM/EDS elemental map of SnSe(TS1) nanoparticles. (a) STEM-BF image (b) Sn map (c) Se map, the scale bar is $100 \mathrm{~nm}$.

(a)

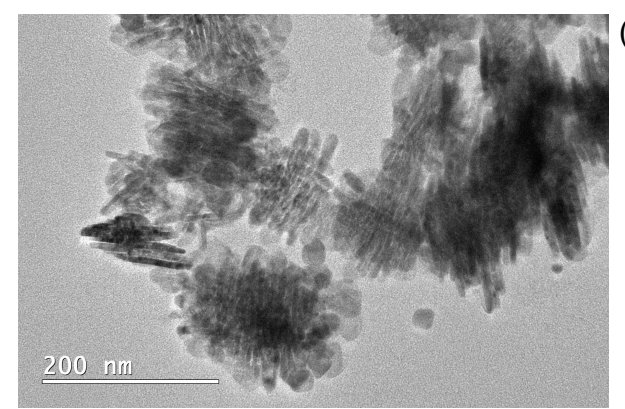

(b)

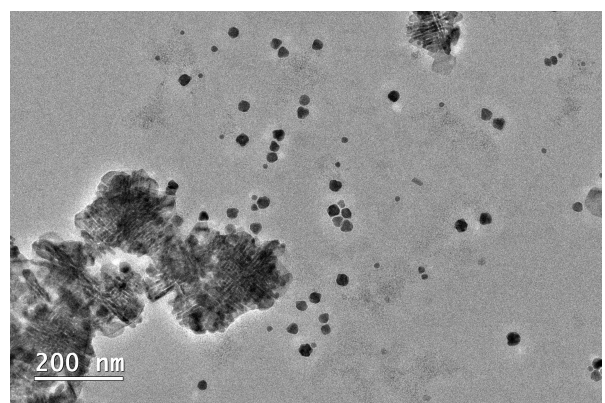

FIGURE 3.36: TEM micrographs of SnSe(TS2) nanoparticles.

(a)

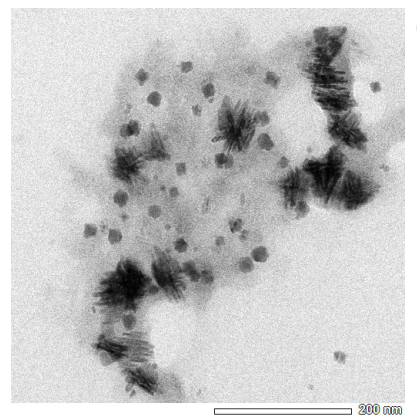

(b)

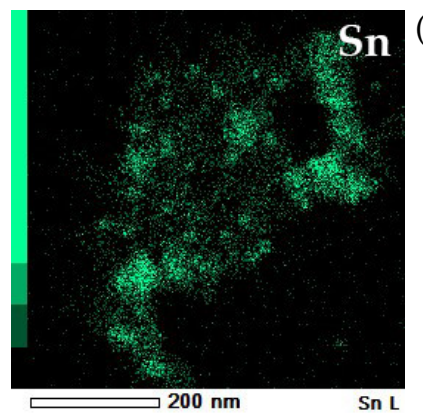

(c)

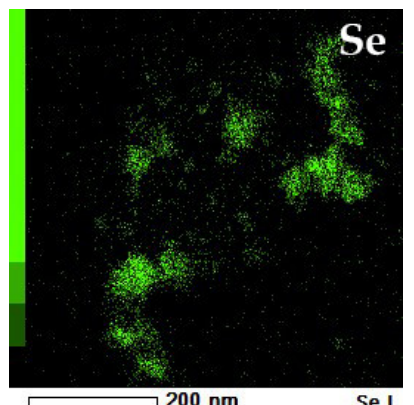

FIGURE 3.37: STEM/EDS elemental map of SnSe(TS2) nanoparticles. (a) STEM-BF image (b) Sn map (c) Se map, the scale bar is $200 \mathrm{~nm}$. 
TABLE 3.12: Composition determined by TEM/EDS for SnSe nanoparticles.

\begin{tabular}{lcc}
\hline Sample & Sn/Se ratio & $\boldsymbol{\sigma}$ for Sn/Se ratio \\
\hline SnSe(TS1) & 1.39 & 0.32 \\
SnSe(TS2) & 0.76 & 0.12 \\
SnSe(TS3) & 0.63 & 0.04 \\
\hline
\end{tabular}

(a)

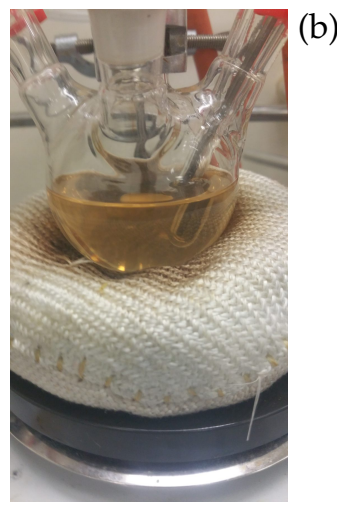

(b)

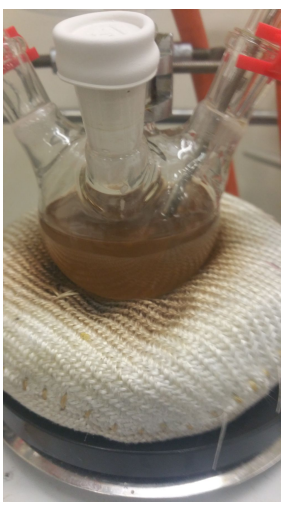

(c)

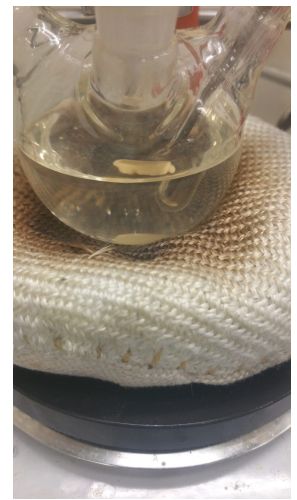

FIGURE 3.38: Quality of Sn precursor after (a) degassing for $120{ }^{\circ} \mathrm{C}$ for 30 mins and (b) after 30 mins at temp $>120{ }^{\circ} \mathrm{C}$ under nitrogen. (c) Sn precursor after degassing using modified temperature increments.

appear to be Sn rich however this may be due to the formation of an additional SnO phase. TEM micrographs and elemental maps of TS2 are shown in Figure 3.36 and Figure 3.37. Here we have formed large aggregates of intercalated nanorods and well dispersed nanoparticles. The elemental maps show a homogeneous distribution of $\mathrm{Sn}$ and Se which suggests that both the nanorods and nanoparticles are SnSe. The morphology of these nanoparticles appears to be significantly different than sub-10 nm SnSe nanoparticles produced by Liu et al. (2014) using a similar method ${ }^{79}$. The differences between our nanoparticles may be due to the quality of the Sn precursor. Observations on the quality of the Sn precursor are shown in Figure 3.38. It was observed that if we degassed the precursor at $>110{ }^{\circ} \mathrm{C}$ for 30 mins then the solution would form a slight yellow colour as depicted in Figure 3.38(a). This was most likely due to the oxidation of tin from residual water/oxygen still present in our reaction. Furthermore it was also observed that after degassing if we left the solution for 30 mins under nitrogen at temps $>120^{\circ} \mathrm{C}$ than we would get the formation of a brown cloudy solution which is indicative of tin oxide forming. Although no SnO phase is observed from the XRD of our SnSe nanoparticles, the presence of $\mathrm{SnO}$ appears to have a significant effect on the morphology of the resulting nanoparticles. 
(a)

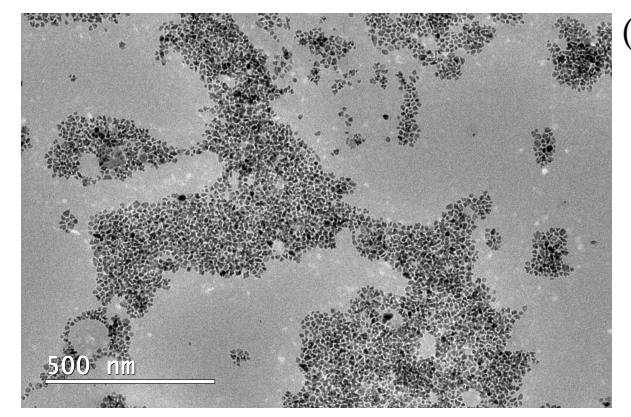

(c)

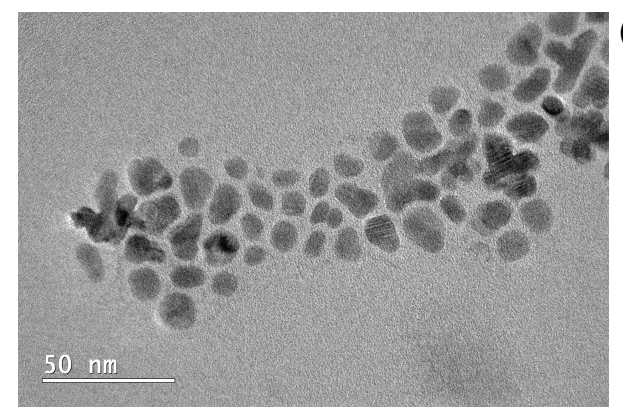

(b)

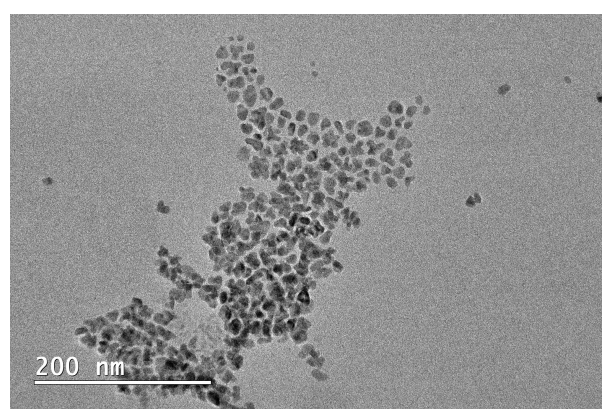

(d)

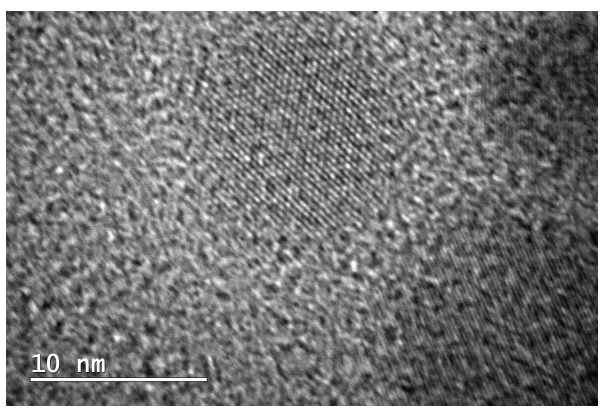

FIGURE 3.39: TEM micrographs of SnSe(TS3) nanoparticles.

(a)

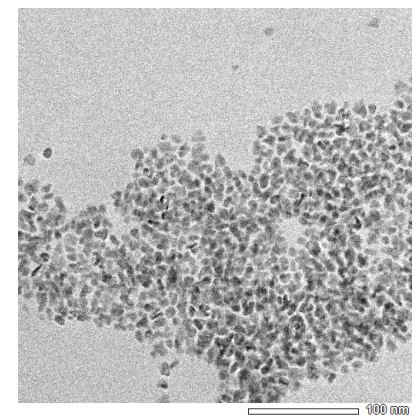

(b)

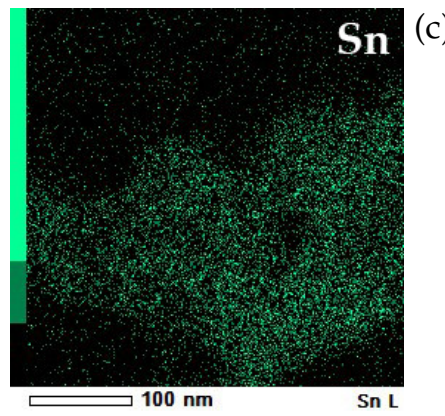

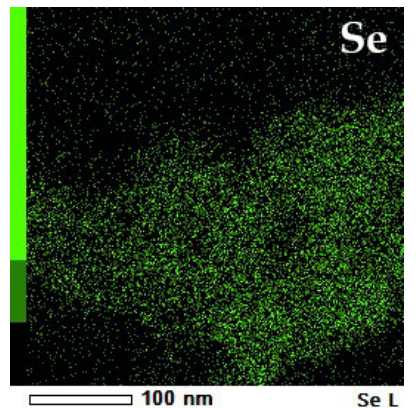

FIGURE 3.40: STEM/EDS elemental map of SnSe(TS3) nanoparticles. (a) STEM-BF image (b) Sn map (c) Se map, the scale bar is $100 \mathrm{~nm}$.

In order to achieve more monodisperse nanoparticles we modify the temperature increase during the degassing phase. In this case our degassing step is done for much longer allowing any residual oxygen or water to be removed. Photographs of the Sn precursor using the modified temperature increments are shown in Figure 3.38(c). The solution is colourless which indicates that no tin oxide has formed. The TEM micrographs and elemental maps of the SnSe (TS3) nanoparticles synthesized using this approach are shown in Figure 3.39 and Figure 3.40. The nanoparticles appear to be much more disperse and are sub-20 nm in size. The morphology and size is comparable to that produced by Liu et al. (2014) using a similar method ${ }^{79}$. The elemental map indicates that we have a uniform distribution of $\mathrm{Sn}$ and Se. The elemental composition is shown in Table 3.12 which indicates that we have made a Se rich nanoparticle. 
Here we have shown a suitable approach to achieving monodisperse sub$20 \mathrm{~nm}$ nanoparticles. Experiments are currently ongoing to produce enough nanoparticles for pellet consolidation and thermoelectric measurements.

\subsection{Summary}

Nanostructured $\mathrm{Bi}_{2} \mathrm{Te}_{3}$ was synthesized via a bismuth(III) oleate precursor. The resulting nanoparticles appeared to be aggregated particles consisting of nanoplates. This is consistent with previous solution synthesized $\mathrm{Bi}_{2} \mathrm{Te}_{3}{ }^{38,39,65,66}$. It was found that different capping ligands such as OLA, DDT and TGA appeared to have no significant effect on the morphology of the solution processed $\mathrm{Bi}_{2} \mathrm{Te}_{3}$. The nanostructured $\mathrm{Bi}_{2} \mathrm{Te}_{3}$ synthesized at $100{ }^{\circ} \mathrm{C}$ and the sample capped with TGA was found to have exceptionally low thermal conductivities of $\sim 0.11 \mathrm{~W} \mathrm{~m}^{-1} \mathrm{~K}^{-1} \sim 0.32 \mathrm{~W} \mathrm{~m}^{-1} \mathrm{~K}^{-1}$ respectively. This is significantly lower then the bulk and nanostructured $\mathrm{Bi}_{2} \mathrm{Te}_{3}$ which has a optimal thermal conductivity of $\sim 0.8 \mathrm{~W} \mathrm{~m}^{-1} \mathrm{~K}^{-1} 2,33,40$. The figure of merit was only $\sim 4 \times 10^{-6}$ for the sample synthesized at $100{ }^{\circ} \mathrm{C}$ and $\sim 1.9 \times 10^{-4}$ for the TGA capped sample. This is relatively low even for nanostructured $\mathrm{Bi}_{2} \mathrm{Te}_{3}$, which is attributed significantly lower electrical conductivities of our nanostructured $\mathrm{Bi}_{2} \mathrm{Te}_{3}$.

Ligand exchange experiments with $\mathrm{S}^{2-}$ and hydrazine were carried out on nanostructured $\mathrm{Bi}_{2} \mathrm{Te}_{3}$. It was found that both ligand exchange methods lead to a increase in the electrical conductivity with the hydrazine treatment increasing by almost an order of magnitude. Aggregated sub-15 nm spherical nanoparticles and rhomboid shaped sub-100 nm nanoparticles of $\mathrm{Bi}_{2} \mathrm{Te}_{3}$ were synthesized using a bismuth nanoparticle precursor approach. The sample synthesized at $120{ }^{\circ} \mathrm{C}$ was found to have a thermopower of $\sim 99 \mu \mathrm{V} \mathrm{K}^{-1}$ and a thermal conductivity of $\sim 0.36 \mathrm{~W} \mathrm{~m}^{-1} \mathrm{~K}^{-1}$ at $300 \mathrm{~K}$. This is an improvement over the thermopower of $\sim 60 \mu \mathrm{V} \mathrm{K}^{-1}$ and thermal conductivity of $\sim 0.8$ $\mathrm{W} \mathrm{m}{ }^{-1} \mathrm{~K}^{-1}$ reported by Scheele et al. (2009) for $\mathrm{Bi}_{2} \mathrm{Te}_{3}$ nanoparticles ${ }^{40}$. After ligand exchange treatment with hydrazine the figure of merit of the sample synthesized at $120{ }^{\circ} \mathrm{C}$ was found to increase by two fold to $\sim 1 \times 10^{-4}$ at room temperature. This was attributed to the increase in the electrical conductivity while the thermal conductivity and thermopower remained largely unaffected. Overall the figure of merit is still low due to electrical conductivity which was found to be $\sim 13.1 \mathrm{~S} \mathrm{~m}^{-1}$ after hydrazine treatment. This is significantly lower than the $\sim 77000 \mathrm{~S} \mathrm{~m}^{-1}$ for $\mathrm{Bi}_{2} \mathrm{Te}_{3}$ nanoparticles ${ }^{40}$.

$\mathrm{Cu}, \mathrm{Ni}$, and $\mathrm{Co}$ doped/alloyed $\mathrm{Bi}_{2} \mathrm{Te}_{3}$ nanoparticles were successfully achieved by using a bismuth nanoparticle precursor approach. The addition 
of copper(II) acetylacetonate resulted in the bismuth precursor changing significantly from a an average size of $41 \mathrm{~nm}$ for the undoped sample to 90 $\mathrm{nm}$ for the doped sample. This inherently had an affect on the resulting $\mathrm{Bi}_{2} \mathrm{Te}$ nanoparticles which formed monodisperse sub $15 \mathrm{~nm}$ particles instead of the sub-100 $\mathrm{nm}$ rhomboid shaped nanoparticles for undoped $\mathrm{Bi}_{2} \mathrm{Te}_{3}$. In the case of $\mathrm{Co}$ and $\mathrm{Ni}$ doped $\mathrm{Bi}_{2} \mathrm{Te}_{3}$ both samples formed similar morphology to the undoped $\mathrm{Bi}_{2} \mathrm{Te}_{3}$. All three doped/alloyed samples showed a decrease in the thermal conductivity relative to undoped $\mathrm{Bi}_{2} \mathrm{Te}_{3}$. This may be attributed to lattice defects leading to phonon-phonon umklapp scattering and scattering from point defects ${ }^{76-78}$. In the case of $\mathrm{Cu}$ and $\mathrm{Ni}$ doped $\mathrm{Bi}_{2} \mathrm{Te}_{3}$ the figure of merit did not increase or change significantly. Further optimisation of the doping concentration may be needed to see improvements in the figure of merit. The Co doped $\mathrm{Bi}_{2} \mathrm{Te}_{3}$ sample saw a an increase in the figure of merit to $\sim 1.4 \times 10^{-4}$ at room temperature. This may be attributed to the improved electrical conductivity and lower thermal conductivity. This suggests that with further optimisation of the doping concentration and the use of post annealing or hot pressing techniques on the pellet may lead to significant increases in the figure of merit.

Although nanostructured $\mathrm{Bi}_{2} \mathrm{Te}_{3}$ showed some improvements with doping/alloying the difficulties in the synthetic control of the morphology and particle size are limiting factors. This brings the need for new novel thermoelectric materials such as nanostructured SnSe or single crystal perovskites. 


\section{Chapter 4}

\section{Synthesis and characterisation of perovskite single crystals}

Organic-inorganic halide single crystal perovskites are starting to be considered for thermoelectric applications due to their low thermal conductivity and high Seebeck coefficient ${ }^{62,63}$, along with computational predictions of high $Z T^{14}$. This has prompted our investigation into single crystal perovskites as a thermoelectric material.

In recent years a large body of research has been focused on organicinorganic mixed halide perovskites due to their remarkable optical and electrical properties, along with being relatively cheap and solution processable. Properties such as balanced electron and hole transport ${ }^{46}$, long diffusion lengths ${ }^{47,48}$, high mobilities ${ }^{49,50}$, and large absorption coefficients in the UV-vis region ${ }^{51}$ have seen perovskites being used for photovoltaics ${ }^{15-18,80}$, light emitting diodes ${ }^{52,53}$, photodetectors ${ }^{54-58}$, and lasers ${ }^{59-61}$. Recently single crystal organic-inorganic halide perovskites have been shown to exhibit significantly longer diffusion lengths, higher mobilities and lower trap densities compared to their polycrystalline thin films ${ }^{81,82}$. These improvements may be attributed to the lack of grain boundaries present in large single crystals, which typically contain a large density of traps ${ }^{83}$. This makes growing large single crystals with a tunable bandgap of particular interest improving device performance over typical thin film based devices.

Typically single crystal perovskites have been grown using cooling induced precipitation ${ }^{56,84}$, seeded solution growth ${ }^{48}$ or antisolvent vapourassisted crystallisation $(\mathrm{VAC})^{82}$, however these methods typically take weeks to prepare high quality crystalline samples. Recently a method for rapid inverse temperature crystallisation (ITC) of large single crystal perovskites has been developed ${ }^{58,81,85}$. This method takes advantage of the observed retrograde solubility of perovskites allowing the rapid growth of high quality millimetre sized perovskites in a matter of days. The tunability of bandgaps 
in $\mathrm{MAPbBr}_{3-\mathrm{x}} \mathrm{Cl}_{\mathrm{x}}$ based perovskites have been shown for large single crystals grown by cooling induced precipitation ${ }^{56}$ and a inverse solubility effect similar to ITC $^{86}$, however mixed halides with a formamidinium cation and mixed metals of $\mathrm{Pb} / \mathrm{Sn}$ have yet to be grown using the rapid ITC method.

In this chapter we present optimised growth conditions to produce large single crystal perovskites grown using ITC. Large single crystals perovskites with mixed $\mathrm{Br} / \mathrm{Cl}$ halides are achieved and the synthetic properties, optical properties, and electrical properties are investigated. Mixed metal perovskites of $\mathrm{Pb} / \mathrm{Sn}$ are also produced and their optical and electrical properties are characterized. The thermoelectric properties were then investigated for $\mathrm{MAPbBr}_{3}$ single crystals.

\subsection{Experimental}

\subsubsection{Materials}

Lead bromide $\left(\mathrm{PbBr}_{2}, \geq 98 \%\right)$, lead chloride $\left(\mathrm{PbCl}_{2}, 98 \%\right)$, dimethyl sulfoxide (DMSO, anhydrous, $\geq 99.9 \%$ ), N,N-dimethylformamide (DMF, anhydrous, $99.8 \%)$, tin(II) bromide $\left(\mathrm{SnBr}_{2}\right)$, dichloromethane (DCM), chloroform $\left(\mathrm{CHCl}_{3}\right)$ and methylamine solution (33 wt. \% in absolute ethanol) were purchased from Sigma-Aldrich. Methylammonium bromide (MABr) and formamidinium bromide (FABr) were purchased from Dyesol Limited. $\gamma$-Butyrolactone (GBL, $99+\%)$ was purchased from Acros Organics. All precursors were used as received without any purification.

\section{2 $\mathrm{MAPbBr}_{3-\mathrm{x}} \mathrm{Cl}_{\mathrm{x}}$ based perovskites}

Here we use this method of ITC to produce large single crystal perovskites with mixed $\mathrm{Br} / \mathrm{Cl}$ halides. Using a method similar to that reported by Saidaminov et al. (2015) and Maculan et al. (2015) $\mathrm{MAPbBr}_{3}$ and $\mathrm{MAPbCl}_{3}$ singe crystals are grown ${ }^{58,81}$. We then develop a method to synthesize $\mathrm{MAPbBr}_{3-\mathrm{x}} \mathrm{Cl}_{\mathrm{x}}$ based single crystal perovskites and the electrical and optical properties are characterized.

\subsubsection{Synthesis}

Although various reaction conditions were attempted to grow organic-inorganic single crystal perovskites using ITC only the optimised reaction conditions 
are reported below.

Synthesis of $\mathrm{MAPbBr}_{3}$. A $1 \mathrm{M}$ solution of $\mathrm{MAPbBr}_{3}$ was prepared by dissolving equal moles of $\mathrm{MABr}$ and $\mathrm{PbBr}_{2}$ in $\mathrm{DMF}$. The solution was then filtered using a $0.22 \mu \mathrm{m}$ hydrophilic PTFE filter. $2 \mathrm{~mL}$ of the filtrate was then added to a glass vial and the solution was then added to an oil bath. The oil bath was slowly heated to $80{ }^{\circ} \mathrm{C}$ and the crystals were grown for 5 hours under ambient conditions (40-60\% humidity). Further growth of the single crystals was then achieved by carefully transferring the single crystals to a fresh growth solution as depicted in Figure 4.1. The growth solution was replaced three times for a total synthesize time of 20 hours.

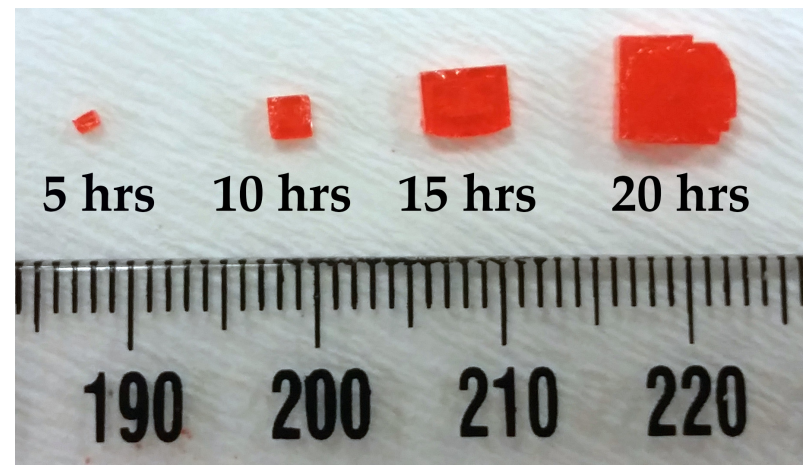

FIGURE 4.1: $\mathrm{MAPbBr}_{3}$ single crystal growth at different time intervals.

Single crystal washing. Once the crystals are removed from the oil bath the excess precursors/growth solution was discarded using a syringe. DCM (antisolvent) was then added upon which a coloured precipitate forms instantly indicating the presence of unreacted perovskite precursors. DMF was then added to the solution to dissolve the precipitate which forms a colourless solution. The excess precursors were then discarded and this process was repeated until the addition of DCM forms a colourless solution. The single crystals were then suspended in DCM to protect the surface from alterations due to prolonged dewetting or exposure to air.

The same general inverse temperature crystallisation method and crystal washing technique for $\mathrm{MAPbBr}_{3}$ was used to grow the following perovskites with these alterations.

Synthesis of $\mathrm{MAPbBr}_{3-\mathrm{x}} \mathrm{Cl}_{\mathrm{x}}$ (nominal $\left.\mathrm{x}=0.5\right)$. A $1 \mathrm{M}$ growth solution of MAPbBr ${ }_{2.5} \mathrm{Cl}_{0.5}$ was prepared by dissolving $\mathrm{MABr} / \mathrm{PbBr}_{2} / \mathrm{PbCl}_{2}$ in a 4:3:1 molar ratio in DMF. The crystals were grown at $60{ }^{\circ} \mathrm{C}$ for 5 hours. The crystals were then transferred to a new growth solutions. The growth solution was replaced 4 times for a total synthesis time of 25 hours. The single crystals were then washed and stored in DCM. 
Synthesis of $\mathrm{MAPbBr}_{3-\mathrm{x}} \mathrm{Cl}_{\mathrm{x}}$ (nominal $\mathrm{x}=1$ ). A $1 \mathrm{M}$ growth solution of $\mathrm{MAPbBr}{ }_{2} \mathrm{Cl}_{1}$ was prepared by dissolving $\mathrm{MABr} / \mathrm{PbBr}_{2} / \mathrm{PbCl}_{2}$ in a 2:1:1 molar ratio in DMF-DMSO (10:1 by volume). The crystals were then grown at $65^{\circ} \mathrm{C}$ for 12 hours. The growth solution was replaced 3 times for a total synthesis time of 48 hours. The single crystals were then washed and stored in DCM.

Synthesis of methylammonium chloride. Methylammonium chloride $(\mathrm{MACl})$ was synthesised from the reaction of hydrochloride acid with methylamine solution (33 wt.\% in absolute ethanol). Methylamine was added to hydrochloric acid in molar ratio of 1.2:1 in an ice bath with stirring for 3 hours. The excess solvents were then evaporated and the resulting material was dried under vacuum for 2 hours at $50{ }^{\circ} \mathrm{C}$. The white powder was then dissolved in methanol and filtered using a $0.22 \mu \mathrm{m}$ PTFE hydrophilic filter. $\mathrm{MACl}$ was then recrystallized using diethyl ether and the crystals were collected and dried under vacuum at $60{ }^{\circ} \mathrm{C}$.

Synthesis of $\mathrm{MAPbCl}_{3} \cdot \mathrm{A} 1 \mathrm{M}$ growth solution of $\mathrm{MAPbCl}_{3}$ was prepared by dissolving equal moles of $\mathrm{MACl}$ and $\mathrm{PbCl}_{2}$ in DMF-DMSO (1:1 by volume). The crystals were then grown at $50{ }^{\circ} \mathrm{C}$ for 12 hours. The growth solution was replaced 3 times for a total synthesis time of 48 hours. The single crystals were then washed and stored in DCM.

\subsubsection{Results and discussion}

Previous reports have already shown the retrograde solubility of $\mathrm{MAPbBr}_{3}$ and $\mathrm{MAPbCl}_{3}$ in DMF and DMF-DMSO (1:1 v/v) respectively ${ }^{58,81}$. It was shown that due to the low solubility of $\mathrm{PbCl}_{2}$ in $\mathrm{DMF}$, a more polar solvent such as DMSO must be used to initiate the retrograde solubility of $\mathrm{MAPbCl}_{3}{ }^{58}$. In order to achieve ITC of $\mathrm{MAPbBr}_{3-\mathrm{x}} \mathrm{Cl}_{\mathrm{x}}$ mixed halides we found that the amount of DMSO present must be tuned with increasing $\mathrm{Cl}$ inclusion. If too much DMSO is present then this would suppress the growth of the perovskite while too little DMSO would result in an insoluble yellow perovskite precipitate forming.

We found that for a nominal $\mathrm{Br} / \mathrm{Cl}$ precursor ratio of $5: 1$, the $\mathrm{PbCl}_{2}$ concentration was low enough that no DMSO was needed to form a $1 \mathrm{M}$ solution and initiate the retrograde solubility of the perovskite. Unlike the case of $\mathrm{MAPbBr}_{3}$ if a growth temperature of $80^{\circ} \mathrm{C}$ was used we observed the formation of lots of small crystals. In order to grow large single crystals the growth temperature must be lowered to $60{ }^{\circ} \mathrm{C}$. In the case of a nominal $\mathrm{Br} / \mathrm{Cl}$ ratio of 2:1, we observed that a 10:1 v/v mixture of DMF-DMSO was needed to prevent 
(a)

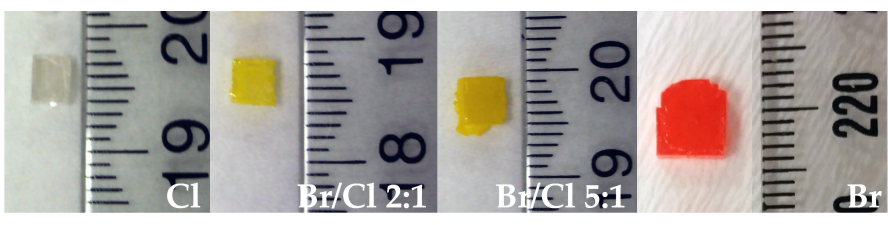

(b)

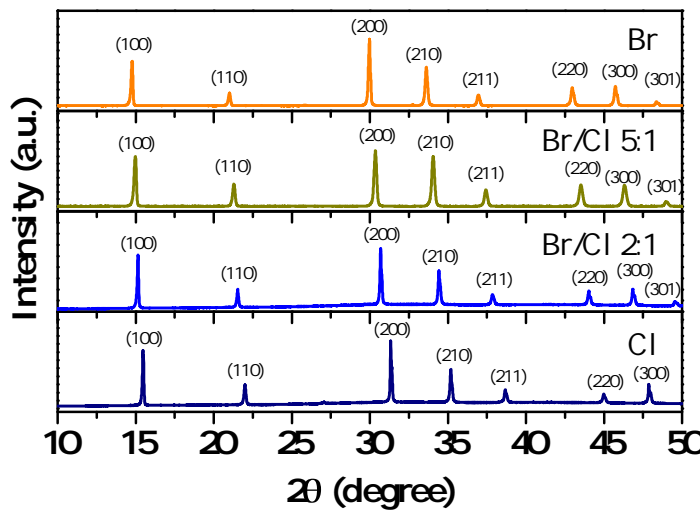

FIGURE 4.2: (a) Photographs of $\mathrm{MAPbBr}_{3-\mathrm{x}} \mathrm{Cl}_{\mathrm{x}}$ based perovskites (b) powder XRD spectra of $\mathrm{MAPbBr}_{3-\mathrm{x}} \mathrm{Cl}_{\mathrm{x}}$ based perovskites.

the formation of a precipitate while enabling the retrograde solubility of the mixed halide perovskite. Furthermore, it was observed that with increasing $\mathrm{PbCl}_{2}$ inclusion the rate of the crystal growth also decreased. Nevertheless large single crystals were still achieved within days.

Photographs of the typical MA based perovskite single crystals with varying nominal $\mathrm{Br} / \mathrm{Cl}$ ratios are shown in Figure 4.2. It can be seen in Figure 4.2(a) that with increasing $\mathrm{Cl}$ content the $\mathrm{MAPbBr}_{3-\mathrm{x}} \mathrm{Cl}_{\mathrm{x}}$ based single crystals changed from orange (pure $\mathrm{Br}$ ) to yellow (nominal ratio of $\mathrm{Br} / \mathrm{Cl}$ 5:1 and 2:1) and finally colourless (pure $\mathrm{Cl}$ ). Powder X-ray diffraction measurements were carried out on the ground single crystals. The XRD spectra are shown in Figure 4.2(b). The pure $\mathrm{Br}$ and $\mathrm{Cl}$ XRD spectra are consistent with previously reported XRD data of the same single crystals ${ }^{58,81,82}$. We can see that the diffractions peaks shift to higher angles with increasing $\mathrm{Cl}$ content thus indicating a decrease in the lattice constant with increasing $\mathrm{Cl}$ inclusion (due to the decreased size of $\mathrm{Cl}$ radii relative to $\mathrm{Br}$ ). The shift in the lattice constant is summarized in Table 4.1 .

To identify the actual composition of halide present in the single crystals energy dispersive X-ray spectroscopy (EDS) was carried out using a scanning electron microscope (SEM). The ratio of $\mathrm{Br} / \mathrm{Cl}$ peak is determined from the average of several different scans on the surface of a single crystal. For each different position we used a scanning area of at least $100 \mu \mathrm{m} \times 100 \mu \mathrm{m}$ and 
TABLE 4.1: $\mathrm{Br} / \mathrm{Cl}$ ratio determined from SEM/EDS, lattice constant determined from powder $\mathrm{XRD}$, and band gap calculated from Tauc plots for $\mathrm{MAPbBr}_{3-\mathrm{x}} \mathrm{Cl}_{\mathrm{x}}$ based perovskites.

\begin{tabular}{lccc}
\hline Nominal ratio & EDS ratio & Lattice constant $(\AA)$ & Band gap (eV) \\
\hline Pure $\mathrm{Br}$ & $\mathrm{N} / \mathrm{A}$ & 5.96 & 2.18 \\
$\mathrm{Br} / \mathrm{Cl} 5: 1$ & $\mathrm{Br} / \mathrm{Cl} 5.5: 1$ & 5.88 & 2.31 \\
$\mathrm{Br} / \mathrm{Cl}: 1$ & $\mathrm{Br} / \mathrm{Cl} 2.1: 1$ & 5.82 & 2.49 \\
Pure $\mathrm{Cl}$ & $\mathrm{N} / \mathrm{A}$ & 5.70 & 2.88 \\
\hline
\end{tabular}

(a)

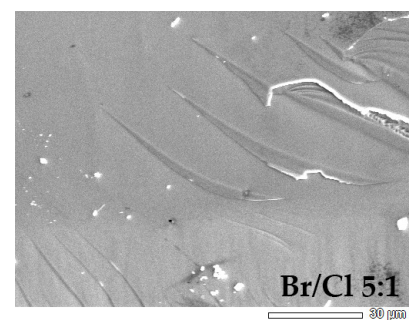

(b)

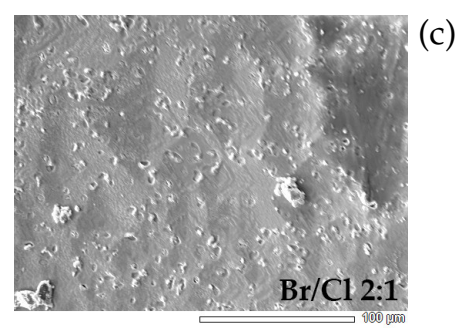

(c)

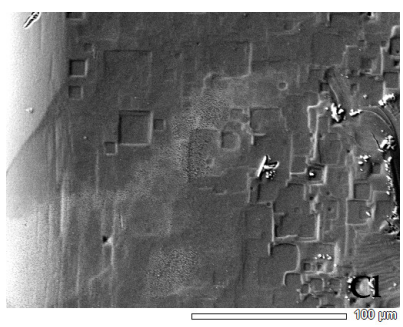

FIGURE 4.3: SEM-SEI images of $\mathrm{MAPbBr}_{3-\mathrm{x}} \mathrm{Cl}_{\mathrm{x}}$ perovskites. (a) nominal $\mathrm{Br} / \mathrm{Cl}$ ratio of 5:1 (b) nominal $\mathrm{Br} / \mathrm{Cl}$ of $2: 1$ (c) pure $\mathrm{Cl}$.

a live time of at least 10 minutes. The halide compositions determined from EDS are summarised in Table 4.1. We can see that the nominal $\mathrm{Br} / \mathrm{Cl}$ ratio for $\mathrm{MAPbBr}_{3-\mathrm{x}} \mathrm{Cl}_{\mathrm{x}}$ based perovskites is very similar to that determined from EDS, with perhaps a slight preferential incorporation of $\mathrm{Br}$ over $\mathrm{Cl}$. The SEM-SEI images of the surface of the single crystal with varying $\mathrm{Cl}$ content are shown in Figure 4.3. We can see that the surface of the single crystals have some surface roughness to them especially for the sample with a nominal $\mathrm{Br} / \mathrm{Cl}$ ratio of 2:1.

The corresponding absorption and PL spectra for $\mathrm{MAPbBr}_{3-\mathrm{x}} \mathrm{Cl}_{\mathrm{x}}$ based single crystals are shown in Figure 4.4. We can see that the absorption edge is gradually blue shifted with increasing $\mathrm{Cl}$ content which matches up with their observed colour changes in Figure 4.2(a). The photoluminescence peak is slightly blue shifted but otherwise shows a similar trend to the absorption spectra. The blue shifted PL emission peak relative to the absorption edge arises due to the surface emissions which is an artefact of the reflection geometry used in our PL experiments whereas the absorption edge is attributed to bulk single crystal due to the transmission geometry used in the UV-vis experiments. This is consistent with other experiments showing the surface edge effects of a single crystal exhibiting a blue shifted fast decaying PL emission relative to the emission from the centre of the single crystals which is dominated by a slow decaying red shifted bulk component ${ }^{87}$.

The $\mathrm{MAPbBr}_{3-\mathrm{x}} \mathrm{Cl}_{\mathrm{x}}$ based single crystals are expected to have a direct band 
(a)

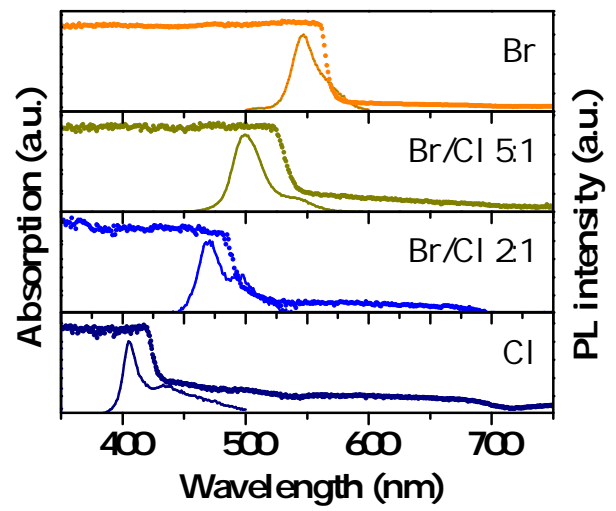

(b)

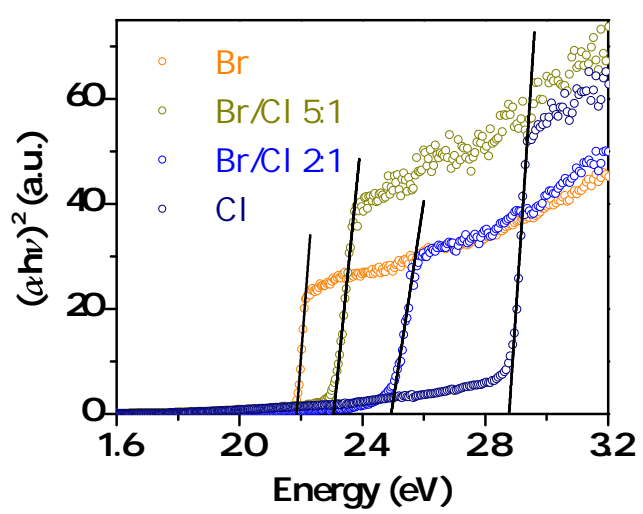

FIGURE 4.4: (a) Steady-state absorption and photoluminescence emission of $\mathrm{MAPbBr}_{3-\mathrm{x}} \mathrm{Cl}_{\mathrm{x}}$ based perovskites. (b) Tauc plots of $\mathrm{MAPbBr}_{3-\mathrm{x}} \mathrm{Cl}_{\mathrm{x}}$ based perovskites.

(a)

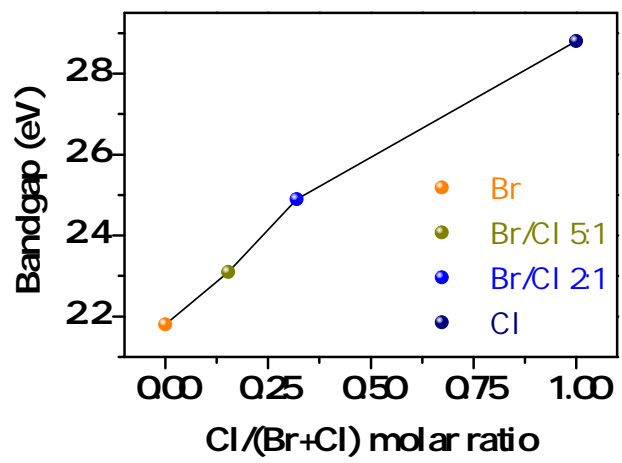

(b)

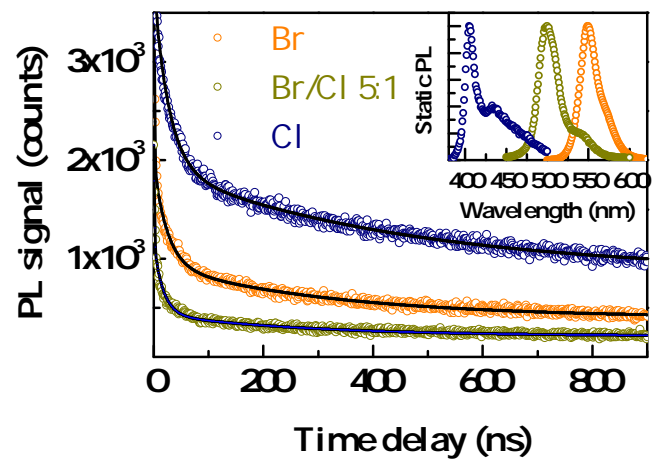

FIGURE 4.5: (a) Bandgap of $\mathrm{MAPbBr}_{3-\mathrm{x}} \mathrm{Cl}_{\mathrm{x}}$ based perovskites as a function of $\mathrm{Cl} /(\mathrm{Br}+\mathrm{Cl})$ molar ratio determined from SEM/EDS (b) PL time decay trace on $\mathrm{MAPbBr}_{3-\mathrm{x}} \mathrm{Cl}_{\mathrm{x}}$ based perovskites. Inset: corresponding PL emission peak.

TABLE 4.2: PL decay lifetimes for $\mathrm{MAPbBr}_{3-\mathrm{x}} \mathrm{Cl}_{\mathrm{x}}$ based perovskites.

\begin{tabular}{lcc}
\hline Nominal ratios & \multicolumn{2}{c}{ PL decay lifetimes (ns) } \\
\cline { 2 - 3 } & $\boldsymbol{\tau}_{\mathbf{1}}$ & $\boldsymbol{\tau}_{\mathbf{2}}$ \\
\hline Pure $\mathrm{Br}$ & $25 \pm 1$ & $321 \pm 8$ \\
$\mathrm{Br} / \mathrm{Cl} \mathrm{5:1}$ & $17 \pm 1$ & $276 \pm 11$ \\
Pure $\mathrm{Cl}$ & $27 \pm 1$ & $448 \pm 11$ \\
\hline
\end{tabular}

gap. The corresponding Tauc plots are shown in Figure 4.4(b). The bandgap as a function of $\mathrm{Cl}$ content is plotted in Figure 4.5(a). We can see that with increasing $\mathrm{Cl}$ content we have a roughly linear increase in the bandgap. This shows us that it is possible to specifically tune the bandgap by altering the ratio of $\mathrm{Cl}$ to $\mathrm{Br}$ present. The $\mathrm{PL}$ decay curves for $\mathrm{MAPbBr}_{3-\mathrm{x}} \mathrm{Cl}_{\mathrm{x}}$ based perovskites are shown in Figure 4.5(b) and the extrapolated time constants using a biexponential fit are summarized in Table 4.2. The sample with a nominal 
ratio of $\mathrm{Br} / \mathrm{Cl}$ 2:1 had a PL emission that was too weak for time correlated single photon counting measurements. The single crystals were observed to have two time components; A fast surface component and a slow bulk component. In the case of $\mathrm{MAPbBr}_{3}$ single crystals the lifetimes were comparable to the reported lifetimes of $\tau \approx 41 \mathrm{~ns}$ and $357 \mathrm{~ns}$ for $\mathrm{MAPbBr}_{3}$ single crystals grown using antisolvent vapour-assisted crystallisation ${ }^{82}$. It appears that the mixed $\mathrm{Br} / \mathrm{Cl}$ single crystal perovskites have shorter lifetimes (surface and bulk component) relative to pure $\mathrm{Br}$ and $\mathrm{Cl}$ single crystals. This may be due to the formation of more trap states for mixed halide samples relative to the pure $\mathrm{Br}$ and $\mathrm{Cl}$ samples. That is to say that an increase in the number of trap states results in a increase in the non-radiative carrier recombination. This reduces the charge carrier density and thus the number of photogenerated carriers and the recombination lifetime.

\subsubsection{Electrical characterisation and modelling}

The current voltage (IV) characteristics of our single crystals were measured in order to extract properties such as mobilities and diffusion lengths to compare with previously reported pure single crystals ${ }^{81,82}$. It should be noted that the thermoelectric properties were not measured due to several experimental difficulties which are discussed in detail in section 4.5. In order to investigate the (IV) characteristics of our single crystal perovskites we investigated two different types of electrode geometries. The models and observations using a sandwich electrode geometry are discussed below followed by a tentative model developed for a planar electrode geometry.

\section{SCLC theory for sandwhich geometry}

The electrical properties of $\mathrm{MAPbBr}_{3}$ were investigated using a sandwich type electrode geometry which is shown in Figure 4.6(a). The $\mathrm{Au} / \mathrm{MAPbBr}_{3} / \mathrm{Au}$ structure was prepared by evaporating rectangular electrodes $(5.5 \mathrm{~mm} \times$ $2 \mathrm{~mm}$ ) of $\mathrm{Au}(100 \mathrm{~nm})$ onto both sides of a $\mathrm{MAPbBr}_{3}$ single crystal with a thickness of $2.83 \mathrm{~mm}$. The IV characteristics were then measured in a dark environment using a probe station connected to a Keithley 4200SCS. This sandwich electrode geometry has been used in previous reports for perovskite single crystal electrical characterisation in which the current voltage characteristics were modelled using space charge limited current (SCLC) theory ${ }^{81,82}$. In this structure a 1D geometry can be considered in which the charge transport is often limited by space charges and the current voltage properties can be 
described by the Mott-Gurney law ${ }^{88}$ :

$$
J=\frac{9}{8} \mu \epsilon_{r} \epsilon_{0} \frac{V^{2}}{l^{3}}
$$

where $\mu$ is the mobility, $\epsilon_{r}$ is the relative permittivity, $\epsilon_{0}$ is the permittivity of free space, $V$ is the voltage, and $l$ is separation of the parallel electrodes. If we have a material which contains traps then an additional modification can be added to the Mott-Gurney law ${ }^{89}$ :

$$
J=\frac{9}{8} \Theta \mu \epsilon_{r} \epsilon_{0} \frac{V^{2}}{l^{3}}
$$

where $\Theta$ is the trapping factor. The trapping factors assume that under SCLC flow and in a dark environment the free and trapped charge carriers are in equilibrium and the density of the trapped charge carriers follow Fermi-Dirac statistics ${ }^{90}$. The trapping factor is given by:

$$
\Theta=\frac{N_{v}}{N_{t}} \exp \left(\frac{-E_{t}}{k_{B} T}\right)
$$

where $N_{t}$ is the trap density and $E_{t}$ is the trap energy. In this model at low voltages the IV characteristics follow ohms law. As we increase the voltage the density of the free charge carriers increase and eventually the current will increase abruptly as all the traps are filled $(\Theta=1)$. This transition is known as the trap free limit, which occurs at the trap free limited voltage $\left(V_{\mathrm{TFL}}\right)$. Once all the traps are filled we enter the SCLC trap free regime in which $I \propto V^{2}$ as described by the Mott-Gurney Law (4.1). The trap free limited voltage can then be related to the trap density by ${ }^{89}$ :

$$
N_{t}=\frac{2 \epsilon_{r} \epsilon_{0} V_{\mathrm{TFL}}}{e l^{2}} .
$$

The trap density can thus be determined by finding $V_{\mathrm{TFL}}$ and the mobility of the charge carriers can be determined from the SCLC trap free regime using Equation 4.1. The IV trace for a sandwich electrode geometry consisting of $\mathrm{Au} / \mathrm{MAPbBr}_{3} / \mathrm{Au}$ with a thickness of $2.83 \mathrm{~mm}$ is shown in Figure 4.6. The SCLC trap free regime and $V_{\mathrm{TFL}}$ was not observed in the IV trace. This is most likely due to the fact that this regime occurs outside our measured voltage range. $\mathrm{MAPbBr}_{3}$ synthesized using ITC has been reported to have a trap density of $N_{t}=3 \times 10^{10} \mathrm{~cm}^{-3} \quad 81$. If we assume our single crystals have a similar trap density than we obtain a trap free limited voltage of $V_{\mathrm{TFL}}=85$ $\mathrm{V}$. This suggests that the SCLC regime is outside of this region furthermore 
(a)

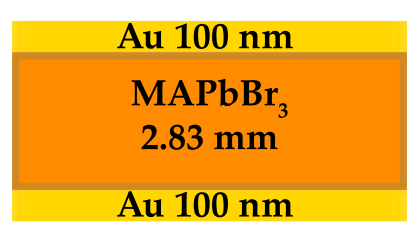

(b)

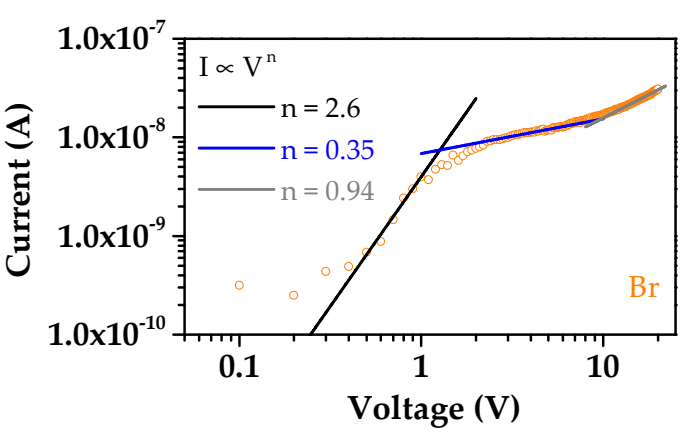

FIGURE 4.6: (a) Sandwich electrode geometry and (b) IV trace of $\mathrm{MAPbbr}_{3}$ using a sandwich electrode geometry in a dark environment.

if our trap density is higher then that reported than this will be shifted to even higher voltages which makes discerning the electrical properties using a sandwich type geometry difficult.

\section{Model for planar geometry}

Here we use a planar electrode geometry to investigate the electrical properties of single crystal perovskites. The device structure is shown in Figure 4.7(a). Although the SCLC theory discussed above is described only for a sandwich electrode geometry it has been shown that this 1D model is still valid for planar contacts if the channel length is smaller than the thickness of the crystal $(l / t<10)^{91}$. If we again assume we have trap density of $N_{t}=3 \times 10^{10} \mathrm{~cm}^{-3}$ then our trap free limited voltage occurs at $V_{\mathrm{TFL}}=0.01 \mathrm{~V}$ for the nominal channel length of $100 \mu \mathrm{m}$ used. This is again outside our measured voltage range which makes it difficult to evaluate our single crystal IV characteristics using SCLC theory.

A planar type electrode structure was prepared by depositing gold pads on the surface of our single crystals via evaporation using shadow masks. The device structure is shown in Figure 4.7(a) and optical microscope image of the deposited gold electrodes is shown in Figure 4.7(b). The nominal channel length was $100 \mu \mathrm{m}$, the width was $2 \mathrm{~mm}$ and $100 \mathrm{~nm}$ of gold was deposited. IV traces were then carried out by contacting the gold electrodes in a dark environment and under a light environment. The light environment for our measurements refers to illumination using a white light LED with an intensity of $45 \mathrm{~W} \mathrm{~m}^{-2}$ (measured using a NIST standardised silicon solar cell) which has the spectrum shown in Figure 4.7(c).

Here we propose a tentative model for extracting mobilities and diffusion lengths from the IV traces acquired using a planar electrode structure. The 
(a)

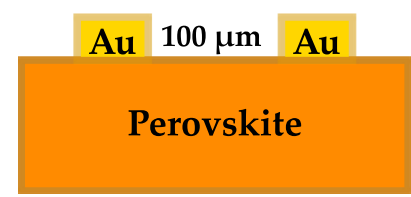

(b)

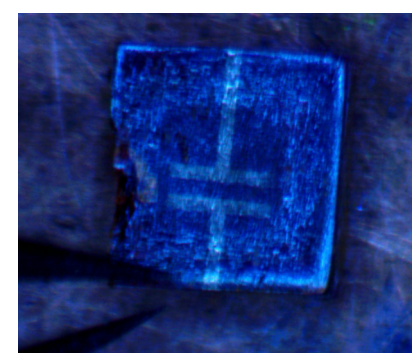

(c)

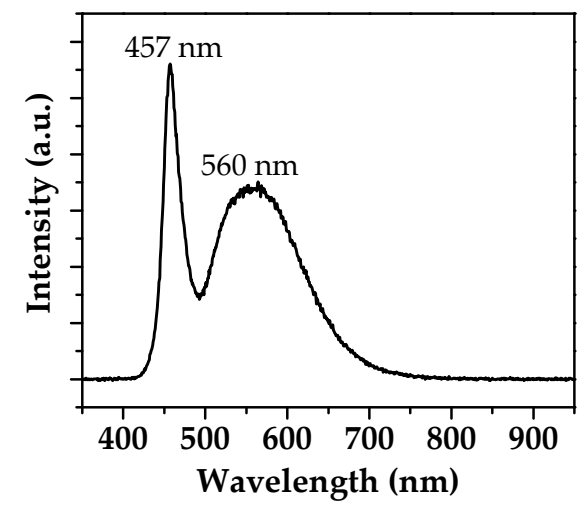

FIGURE 4.7: (a) Device structure used to measure IV traces and (b) optical microscope image of deposited electrodes. (c) White light LED emission spectrum used for illumination of perovskites.

(a)

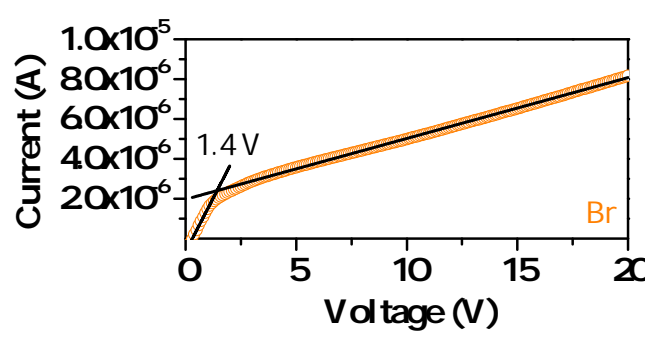

(c)

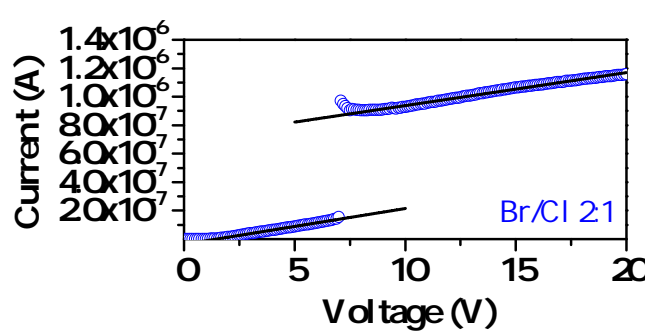

(b)

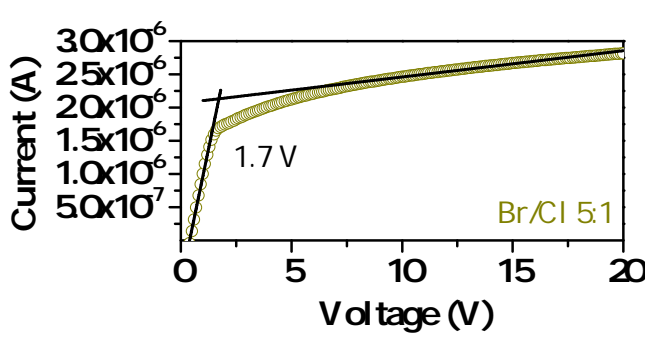

(d)

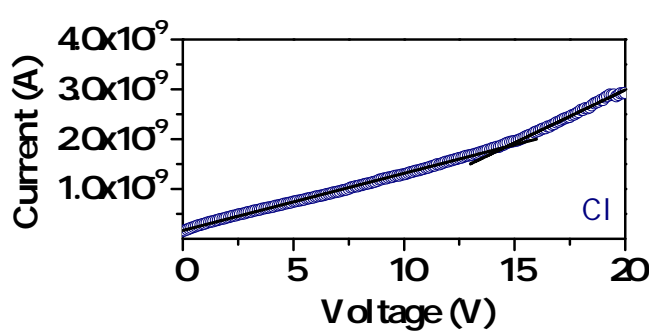

FIGURE 4.8: IV trace for (a) $\mathrm{MAPbBr}_{3}$ (b) nominal $\mathrm{Br} / \mathrm{Cl}$ 5:1 (c) nominal $\mathrm{Br} / \mathrm{Cl}$ 2:1 and (d) $\mathrm{MAPbCl}_{3}$ single crystals under white light illumination of $45 \mathrm{~W} \mathrm{~m}^{-2}$.

IV characteristics of $\mathrm{MAPbBr}_{3-\mathrm{x}} \mathrm{Cl}_{\mathrm{x}}$ based perovskites under white light illumination are shown in Figure 4.8. In the case of $\mathrm{MAPbBr}_{3}$ we can see two distinct linear regions for the IV trace. In the first region at low voltages the drift velocity of the charge carriers is small and the diffusion currents of the 
photogenerated charge carrier dominates. The diffusion length is related to the mobility by:

$$
L_{D}=\sqrt{\frac{k_{B} T}{e \mu \tau}}
$$

where $\mu$ is the mobility and $\tau$ is the carrier lifetime. This means in this low voltage region the photo generation of charge carriers dominates and the electron diffusion length is smaller than the recombination length, which leads to a region dominated by a recombination dependant mobility. The drift velocity is dependent on the voltage $\left(v_{d}=\mu E\right)$. This means as we increase the voltage the electron diffusion length increases. At high voltages the IV characteristics are then dominated by the intrinsic perovskite resistance as the electron diffusion length becomes larger than the recombination length. This means all the generated electrons are extracted and the mobility is dominated by the intrinsic electron mobility of the single crystal as the electron diffusion length is greater than the recombination length. The electrical conductivity is related to the mobility by:

$$
\sigma=\mu n e
$$

where $n$ is the charge carrier concentration which denotes the number of charge carriers per unit volume. The electrical conductivity is an intrinsic property of a material which can be related to the conductance by:

$$
\sigma=G \frac{l}{A}=\mu n e
$$

where $G=1 / R$ is the conductance, $l$ is the path length of the charge carrier, and $A$ is the cross sectional area. Using the definition of charge carrier concentration we obtain:

$$
\mu N e=G l^{2}
$$

Substituting in Ohm's law $(V=I R)$ and using the definition for current $(I=Q / t)$ we can obtain an expression for the mobility:

$$
\mu=\frac{l^{2}}{V t}
$$

where $t$ is the transit time of the charge carriers between two electrodes and $\mathrm{V}$ is the bias between the electrodes. As the recombination length is constant with increasing voltage then at intersection between the two linear regions the diffusion length of the charge carriers must be equal to the recombination length, where the mobility is given by the recombination dependent mobility 
$\left(\mu_{R}\right)$. If we assume that at this intersection the recombination lifetime is equal to the transit time then the recombination dependent mobility can be given by:

$$
\mu_{R}=\frac{l^{2}}{V_{I} \tau}
$$

where $l$ is the channel length, $V_{I}$ is the voltage at the intersection of the two regions and $\tau$ is the recombination lifetime. If we assume that the conductance in each region is dependent on only the mobilities in each region then the ratio can be taken and the mobility $\mu$ (assuming $\mu \approx \mu_{n} \approx \mu_{p}$ which are the electron and hole mobility respectively ${ }^{92,93}$ ) can be directly related to the conductance in each region:

$$
\frac{\mu}{\mu_{R}}=\frac{G_{H}}{G_{L}}
$$

where $\mu_{R}$ is the recombination dependant mobility and $G_{H}$ and $G_{L}$ are the conductances in the high and low voltage regions respectively. Combining Equation 4.11 and Equation 4.10 gives:

$$
\mu=\frac{G_{H} l^{2}}{G_{L} V_{I} \tau}
$$

which allows us to estimate a mobility of the single crystal perovskites using our planar geometry. The best and worst case diffusion lengths can then be estimated using the fast (surface) and slow (bulk) decay lifetimes shown in Table 4.2. If we combine Equation 4.5, Equation 4.11, and Equation 4.10 we obtain an expression for the diffusion length:

$$
L_{D}=\sqrt{\frac{k_{B} T}{q} \frac{l^{2} G_{H}}{V_{I} G_{R}}}
$$

Best case and worst case diffusion lengths can then be estimated by propagating the uncertainties in the nominal channel length, the intersection voltage, and the uncertainties found from $\max / \mathrm{min}$ linear fits of the IV traces. Estimations for the mobilities and diffusion lengths for $\mathrm{MAPbBr}_{3-x} \mathrm{Cl}_{x}$ based perovskites are shown in Table 4.3 along with the values reported for $\mathrm{MAPbBr}_{3}$ determined using SCLC theory and a sandwich geometry ${ }^{81,82}$. Note for a nominal precursor ratio of $\mathrm{Br} / \mathrm{Cl} 2: 1$ and $\mathrm{MAPbCl}_{3}$ the mobilities and diffusion length could not be determined as there does not appear to be two distinct linear regions. In the case of $\mathrm{MAPbCl}_{3}$ the model does not apply due to the white light emission being below the band gap of the single crystal. For the nominal $\mathrm{Br} / \mathrm{Cl}$ 2:1 sample the graph in Figure 4.8(c) shows a jump in the 
TABLE 4.3: Extracted mobilities and diffusion lengths for $\mathrm{MAPbBr}_{3-\mathrm{x}} \mathrm{Cl}_{\mathrm{x}}$ based perovskites.

\begin{tabular}{lcc}
\hline Nominal ratio & Mobilities $\left(\mathrm{cm}^{2} \mathrm{~V}^{-1} \mathrm{~s}^{-1}\right)$ & Diffusion length $(\mu \mathrm{m})$ \\
\hline $\mathrm{Br}$ & $30-390$ & $2-8$ \\
$\mathrm{Br} / \mathrm{Cl} 5: 1$ & $10-110$ & $1-4$ \\
$\mathrm{Br}(\mathrm{ITC})^{* 81}$ & 24 & $1.3-4.3$ \\
$\mathrm{Br}(\mathrm{VAC})^{* 82}$ & $20-114$ & $3-17$ \\
\hline
\end{tabular}

${ }^{*}$ Estimation of mobilities and diffusion lengths reported from literature are also included. In the case of the sample grown using ITC the mobilities were calculated using SCLC theory and best/worst case diffusion lengths were estimated from the PL decay lifetimes ${ }^{81}$. In the case of the sample grown using antisolvent vapor-assisted crystallisation (VAC) the mobilities were estimated from hall effect measurements, transit time measurements and SCLC theory. The diffusion lengths were then estimated from the best/worst case mobilities and PL decay lifetimes ${ }^{82}$.

voltage which may be due to contacting issues. The diffusion length and mobilities determined for $\mathrm{MAPbBr}_{3}$ were found to be between 2-8 $\mu \mathrm{m}$ and $30-390 \mathrm{~cm}^{2} \mathrm{~V}^{-1} \mathrm{~s}^{-1}$ respectively. This is comparable to the diffusion lengths of 1.3-4.3 $\mu \mathrm{m}$ and mobilities of $24 \mathrm{~cm}^{2} \mathrm{~V}^{-1} \mathrm{~s}^{-1}$ determined using SCLC theory for $\mathrm{MAPbBr}_{3}$ single crystals grown with $\mathrm{ITC}^{81}$.

It should be noted that both SCLC theory and our tentative model assumes that there is only one dominant charge carrier present which may not be true $\mathrm{g}^{9293}$. Both models also ignore and fail to account for effects such as self healing of defects and the mechanism of ion diffusion which are both known to occur in single crystal perovskite materials ${ }^{94-99}$.

Another possible explanation for the observed changes in the IV traces and hence the mobility at high voltages for $\mathrm{MAPbBr}_{3-\mathrm{x}} \mathrm{Cl}_{\mathrm{x}}$ single crystals might be due to reaching the saturation velocity. Typically the drift velocity in a material is proportional to the electric field strength, however at high electric field strengths we can reach a saturation velocity resulting in non linear behaviour where the mobility is now dependant on the electric field strength. Typical electric fields strengths for velocity saturation in semiconductors occurs on the order of $10-100 \mathrm{kV} \mathrm{cm}^{-1}{ }^{100}$. This corresponds to voltages of $100-1000 \mathrm{~V}$ for a channel length of $100 \mu \mathrm{m}$ which suggests that we are not likely observing this effect as this region is outside our voltage range.

The IV traces under a dark and light environment are shown in Figure 4.9 and graphs of the on/off ratio at a $2 \mathrm{~V}$ bias are shown in Figure 4.10(a) and summarized in Table 4.4. The on/off ratios are exceptionally high for the samples with a bandgap above the white light emission spectrum. A rough estimate on how successful bandgap tunability of $\mathrm{MAPbBr}_{3-\mathrm{x}} \mathrm{Cl}_{\mathrm{x}}$ perovskites 
(a)

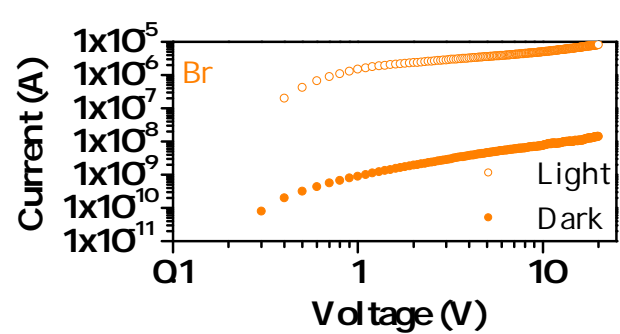

(c)

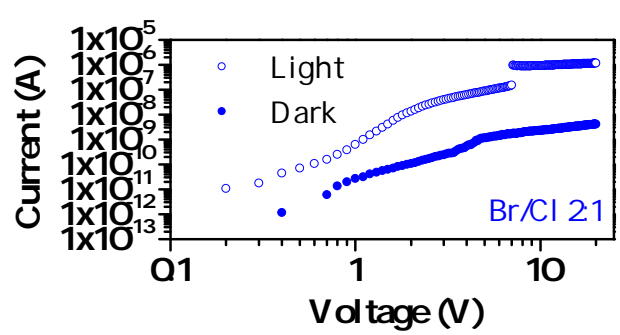

(b)

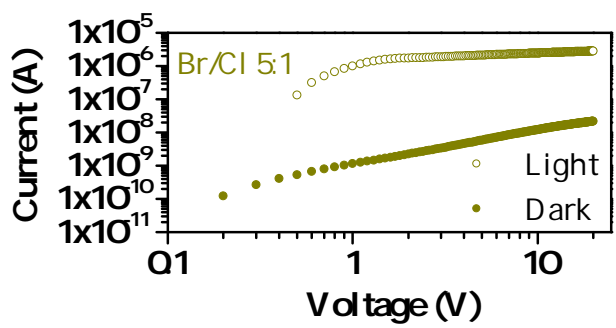

(d)

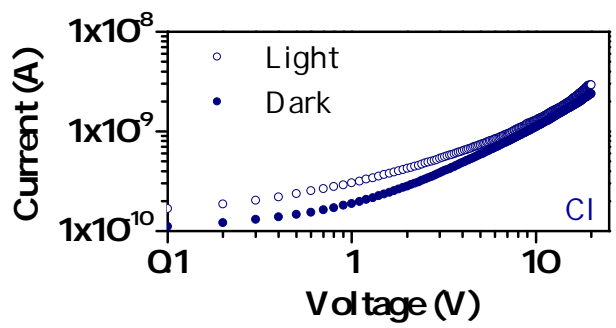

FIGURE 4.9: IV trace for (a) $\mathrm{MAPbBr}_{3}$ (b) nominal $\mathrm{Br} / \mathrm{Cl}$ 5:1 (c) nominal $\mathrm{Br} / \mathrm{Cl} 2: 1$ and (d) $\mathrm{MAPbCl}_{3}$ single crystals in a dark environment and under white light illumination of $45 \mathrm{~W} \mathrm{~m}^{-2}$.

TABLE 4.4: On/off ratio at a $2 \mathrm{~V}$ bias under white light illumination of $45 \mathrm{~W} \mathrm{~m}^{-2}$ for $\mathrm{MAPbBr}_{3-\mathrm{x}} \mathrm{Cl}_{\mathrm{x}}$ based perovskites.

\begin{tabular}{lc}
\hline Nominal ratio & On/Off ratio \\
\hline $\mathrm{Br}$ & 1200 \\
$\mathrm{Br} / \mathrm{Cl} 5: 1$ & 620 \\
$\mathrm{Br} / \mathrm{Cl} 5: 1$ & 55 \\
$\mathrm{Cl}$ & 1.4 \\
\hline
\end{tabular}

can be shown by comparing the on/off ratios to the LED emission spectrum. If we assume the single crystals have a similar absorption depth (typically several $100 \mathrm{~nm}$ for perovskites) then we can compare the trend of the on/off ratio under white light illumination which is depicted in Figure 4.9. The total counts below the absorption edge of the corresponding figures is found by integrating the area under the emission spectrum shown in Figure 4.7(c). The total counts below the band edge is then normalized with respect to the band gap of $\mathrm{MAPbBr}_{3}$. We can see that there is a roughly linear trend for the normalized counts below the LED emission spectrum below. The normalized on/off ratio appear to also have a linear trend for the samples with bandgaps within the emission spectrum of the LED although the slope appears to be more steep perhaps indicating the formation of more trap states with increasing $\mathrm{Cl}$ inclusion. Nevertheless we can see the potential of using $\mathrm{MAPbBr}_{3-\mathrm{x}} \mathrm{Cl}_{\mathrm{x}}$ based perovskites as photodetectors with various wavelengths. 
(a)

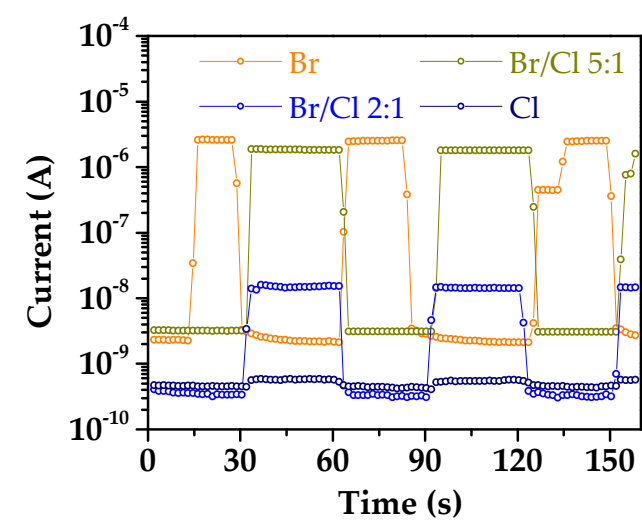

(b)

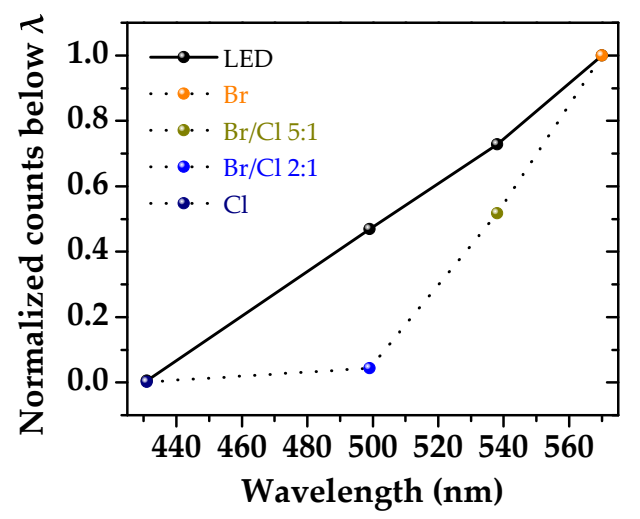

FIGURE 4.10: (a) On/off current at a $2 \mathrm{~V}$ bias under white light illumination of $45 \mathrm{~W} \mathrm{~m}^{-2}$ and (b) comparison of on/off ratio with white light LED emission spectrum for $\mathrm{MAPbBr}_{3-\mathrm{x}} \mathrm{Cl}_{\mathrm{x}}$ based perovskites.

\section{3 $\mathrm{FAPbBr}_{3-\mathrm{x}} \mathrm{Cl}_{\mathrm{x}}$ based perovskites}

\subsubsection{Synthesis}

The same general ITC method and crystal washing technique for $\mathrm{MAPbBr}_{3}$ was used to grow the following perovskites with these alterations. Note that $\mathrm{FAPbbBr}_{3}$ was grown using a similar method to that reported by Saidaminov et al. (2015). As far as I know this is the first reported case of using ITC to grow mixed $\mathrm{Br} / \mathrm{Cl}$ single crystals.

Synthesis of FAPbBr3. A $1 \mathrm{M}$ growth solution of $\mathrm{FAPbBr}_{3}$ was prepared by dissolving equal moles of FABr and $\mathrm{PbBr}_{2}$ in DMF-GBL 1:1 (v/v). The crystals were grown at $60{ }^{\circ} \mathrm{C}$ for 5 hours. The growth solution was replaced 3 times for a total synthesis time of 20 hours. The single crystals were then washed and stored in DCM.

Synthesis of $\mathrm{FAPbBr}_{3-\mathrm{x}} \mathrm{Cl}_{\mathrm{x}}$ (nominal $\mathrm{x}=0.5$ ). A $1 \mathrm{M}$ growth solution of FAPbBr ${ }_{2.5} \mathrm{Cl}_{0.5}$ was prepared by dissolving $\mathrm{FABr} / \mathrm{PbBr}_{2} / \mathrm{PbCl}_{2}$ in a 4:3:1 molar ratio in DMF-GBL 1:1 (v/v). The crystals were then grown at $50{ }^{\circ} \mathrm{C}$ for 12 hours under ambient conditions. The growth solution was replaced 3 times for a total synthesis time of 48 hours. The single crystals were then washed and stored in DCM.

Synthesis of $\mathrm{FAPbBr}_{3-\mathrm{x}} \mathrm{Cl}_{\mathrm{x}}$ (nominal $\mathrm{x}=1$ ). A $0.8 \mathrm{M}$ growth solution of FAPbBr ${ }_{2} \mathrm{Cl}_{1}$ was prepared by dissolving $\mathrm{FABr} / \mathrm{PbBr}_{2} / \mathrm{PbCl}_{2}$ in a 2:1:1 molar ratio in DMF-GBL 1:1 ()v/v). The crystals were grown at $50{ }^{\circ} \mathrm{C}$ for 12 hours. The growth solution was replaced 4 times for a total synthesis time of 60 hours. The single crystals were then washed and stored in DCM. 
Synthesis of $\mathrm{FAPbBr}_{3-\mathrm{x}} \mathrm{Cl}_{\mathrm{x}}$ (nominal $\mathrm{x}=1.5$ ). A $0.5 \mathrm{M}$ growth solution of FAPBr ${ }_{1.5} \mathrm{Cl}_{1.5}$ was prepared by dissolving $\mathrm{FABr} / \mathrm{PbBr}_{2} / \mathrm{PbCl}_{2}$ in a 4:1:3 molar ratio in DMF-GBL 1:1 (v/v). The crystals were then grown at $50{ }^{\circ} \mathrm{C}$ for 12 hours under ambient conditions. The growth solution was replaced 4 times for a total synthesis time of 60 hours. The single crystals were washed and then stored in DCM.

Comparison with different total synthesis times. $\mathrm{FAPbBr}_{3-\mathrm{x}} \mathrm{Cl}_{\mathrm{x}}$ based single crystals with nominal ratios of $x=0.5, x=1$, and $x=1.5$ were also synthesized as per the above conditions however the total synthesis time was reduced to 20 hours. $\mathrm{FAPbBr}_{3}$ was also synthesized with a total synthesis time of 48 hours.

\subsubsection{Results and discussion}

$\mathrm{FAPbBr}_{3}$ single crystals have already been reported to grow via ITC using a 1:1 (v/v) mixture of DMF-GBL ${ }^{85}$. We found that in order to grow $\mathrm{FAPbBr}_{3-\mathrm{x}} \mathrm{Cl}_{\mathrm{x}}$ single crystals a 1:1 (v/v) mixture of DMF-GBL can still be used, however due to the lower solubility of $\mathrm{PbCl}_{2}$ in DMF the concentration and crystallisation temperature must be decreased with the increasing $\mathrm{PbCl}_{2}$ inclusion to optimise the crystal growth. Using the optimised reaction conditions large single crystals of $\mathrm{FAPbBr}_{3-\mathrm{x}} \mathrm{Cl}_{\mathrm{x}}$ were grown in a matter of days. Photographs of the

(a) Totalsynthesis time $=20 \mathrm{hrs}$

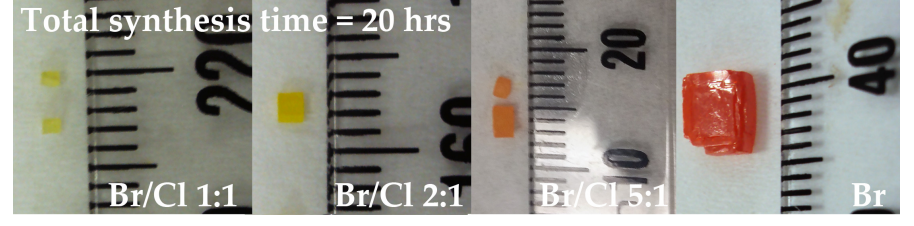

(b)

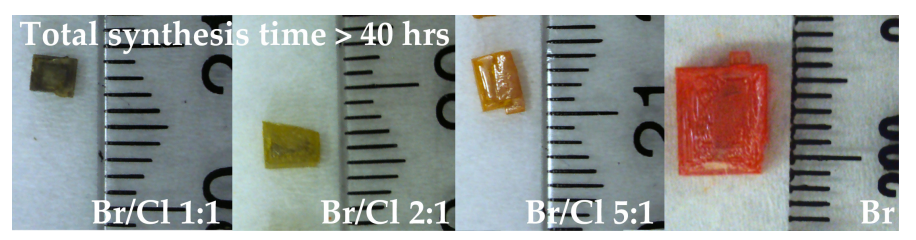

FIGURE 4.11: Photographs of $\mathrm{FAPbBr}_{3-\mathrm{x}} \mathrm{Cl}_{\mathrm{x}}$ based perovskites with a total synthesis time of (a) 20 hours and (b) $>40$ hours.

single crystals with a total synthesis time of 20 hours and $>40$ hours can be found in Figure 4.11. It was observed that for reactions with a total growth time of 20 hours the resulting single crystals were orange, yellow and pale yellow for nominal $\mathrm{Br} / \mathrm{Cl}$ ratios of 5:1, 2:1 and 1:1 respectively. However, if the total synthesis time was increased to $>40$ hours the single crystals were then observed to be more orange/red than their counterparts synthesised for 20 hours. 
(a)

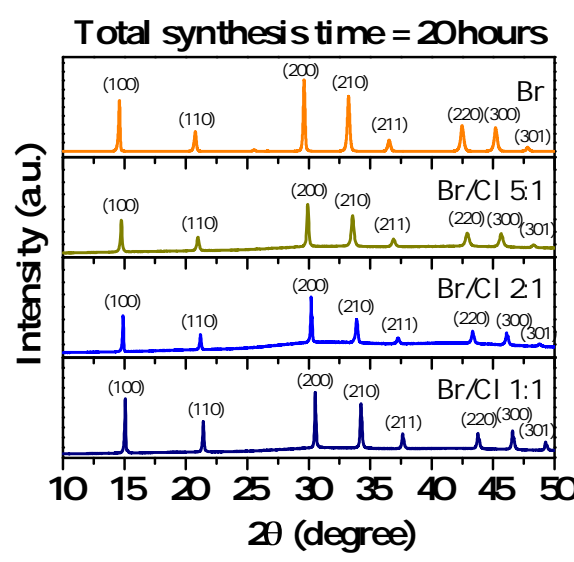

(b)

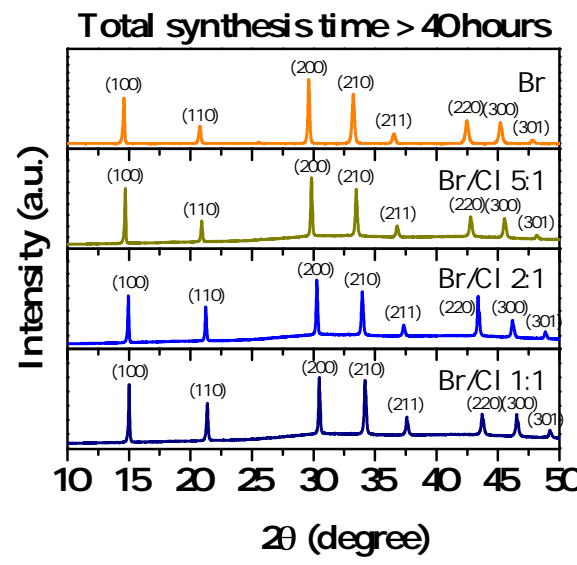

(c)

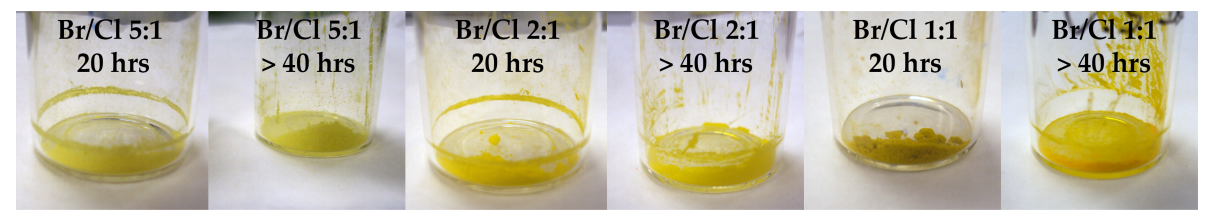

FIGURE 4.12: Powder XRD spectra of $\mathrm{FAPbBr}_{3-\mathrm{x}} \mathrm{Cl}_{\mathrm{x}}$ based perovskites with a total synthesis time of (a) 20 hours and (b) $>$ 40 hours. (c) Photographs of ground $\mathrm{FAPbBr}_{3-\mathrm{x}} \mathrm{Cl}_{\mathrm{x}}$ based perovskites with a total synthesis time of 20 hours and $>40$ hours.

Powder XRD spectra were collected for samples with a total synthesis time of 20 hours and a total synthesis time $>40$ hours and are shown Figure 4.12. The XRD spectra showed no differences between the samples with the same nominal $\mathrm{Br} / \mathrm{Cl}$ ratio and similar lattice constants are found irrespective of the total synthesis time, which are summarized in Table 4.5. With increasing $\mathrm{Cl}$ inclusion the XRD peaks are observed to shift to higher angles which is consistent with $\mathrm{Cl}$ having a smaller radius than $\mathrm{Br}$ thus resulting in a smaller lattice. Photographs of ground $\mathrm{FAPbBr}_{3-\mathrm{x}} \mathrm{Cl}_{\mathrm{x}}$ based single crystal perovskites used for powder XRD are shown in Figure 4.12(c). Upon grinding the samples for XRD the resulting powders were observed to have similar colours of pale yellow, yellow and bright orange/yellow for nominal $\mathrm{Br} / \mathrm{Cl}$ ratios of 5:1, 2:1 and 1:1 respectively. Regardless of the total synthesis time the ground powders appear to have a similar colour contrary to the observations seen Figure 4.11.

EDS elemental composition of the halide ratios are summarized in Table 4.5 and in general appear to show a higher $\mathrm{Br} / \mathrm{Cl}$ ratio than the nominal $\mathrm{Br} / \mathrm{Cl}$ ratios. This indicates the preferential inclusion of $\mathrm{Br}$ over $\mathrm{Cl}$. This is further confirmed by the higher $\mathrm{Br} / \mathrm{Cl}$ ratios for the samples with an increased reaction time. SEM-SEI images of single crystal surface are shown in Figure 4.13. In general the surface appears to be less rough with increased $\mathrm{Cl}$ inclusion 
TABLE 4.5: $\mathrm{Br} / \mathrm{Cl}$ ratio determined from SEM/EDS, lattice constant determined from powder XRD, and bandgap calculated from Tauc plots for $\mathrm{FAPbBr}_{3-\mathrm{x}} \mathrm{Cl}_{\mathrm{x}}$ based perovskites

\begin{tabular}{|c|c|c|c|}
\hline Nominal ratio & EDS ratio & Lattice constant $(\AA)$ & Band gap $(\mathrm{eV})$ \\
\hline Pure Br (20 hrs) & $\mathrm{N} / \mathrm{A}$ & 6.03 & 2.15 \\
\hline $\mathrm{Br} / \mathrm{Cl}$ 5:1 (20 hrs) & $\mathrm{Br} / \mathrm{Cl} 7.8: 1$ & 5.97 & 2.23 \\
\hline $\mathrm{Br} / \mathrm{Cl}$ 2:1 (20 hrs) & $\mathrm{Br} / \mathrm{Cl} 2.4: 1$ & 5.91 & 2.40 \\
\hline $\mathrm{Br} / \mathrm{Cl}$ 1:1 (20 hrs) & $\mathrm{Br} / \mathrm{Cl} 0.95: 1$ & 5.85 & 2.57 \\
\hline Pure Br (> 40 hrs) & $\mathrm{N} / \mathrm{A}$ & 6.03 & 2.15 \\
\hline $\mathrm{Br} / \mathrm{Cl}$ 5:1 (> 40 hrs) & $\mathrm{Br} / \mathrm{Cl} 8.4: 1$ & 5.98 & 2.24 \\
\hline $\mathrm{Br} / \mathrm{Cl} 2: 1$ (> 40 hrs) & $\mathrm{Br} / \mathrm{Cl} 2.9: 1$ & 5.90 & 2.39 \\
\hline $\mathrm{Br} / \mathrm{Cl} 1: 1$ (> 40 hrs) & $\mathrm{Br} / \mathrm{Cl} 1.0: 1$ & 5.86 & $\mathrm{~N} / \mathrm{A}^{*}$ \\
\hline
\end{tabular}

${ }^{*}$ No absorption edge was observed for this sample. This is most likely due to the surface degradation of the single crystal.

and increased total synthesis time.

(a) Total synthesis time $=20$ hours
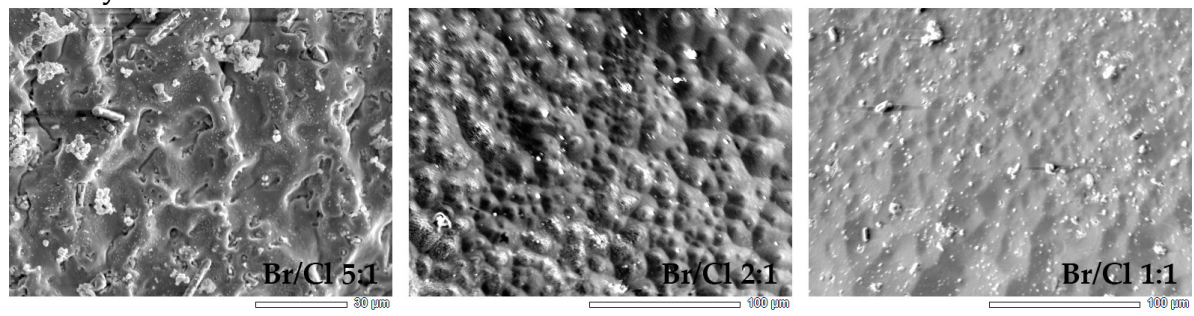

(b) Total synthesis time $>40$ hours

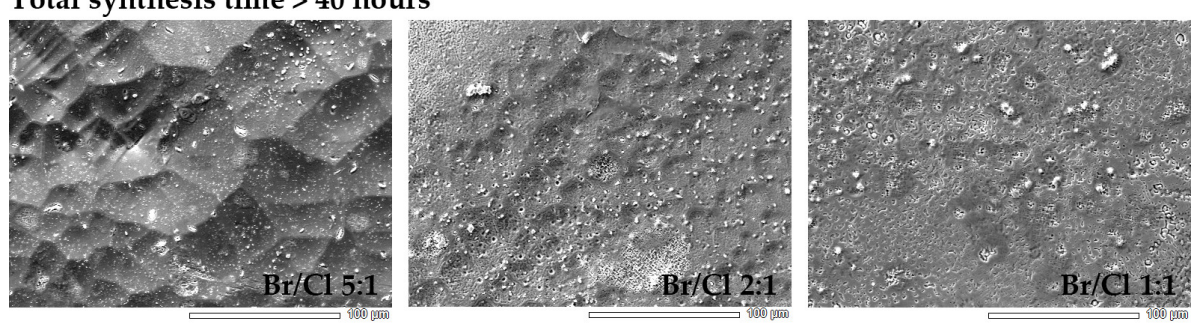

FIGURE 4.13: SEM-SEI images of $\mathrm{FAPbBr}_{3-\mathrm{x}} \mathrm{Cl}_{\mathrm{x}}$ perovskites. (a) total synthesis time $=20$ hours and $(\mathrm{b})$ total synthesis time $>40$ hours.

UV-vis absorption and PL spectra of $\mathrm{FAPbBr}_{3-\mathrm{x}} \mathrm{Cl}_{\mathrm{x}}$ based perovskites with a total synthesis time of 20 hours and $>40$ hours are presented in Figure 4.14 along with corresponding Tauc plots. The absorption edge and the corresponding extrapolated bandgaps of the single crystals appear to be blue shifted with increasing $\mathrm{Cl}$ content and follow the trend observed in Figure 4.11(a) for samples with a total synthesis time of 20 hours. The band gap and absorption edge for samples with the same nominal $\mathrm{Br} / \mathrm{Cl}$ ratio are similar suggesting that the observed differences between the total synthesis time is a surface effect as the 
(a)

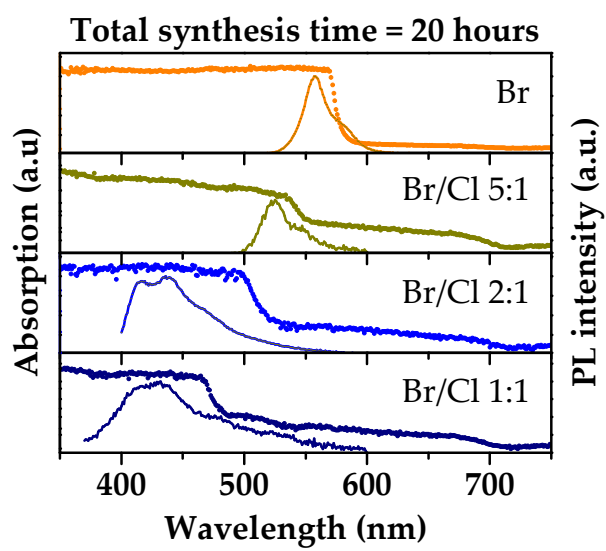

(c)

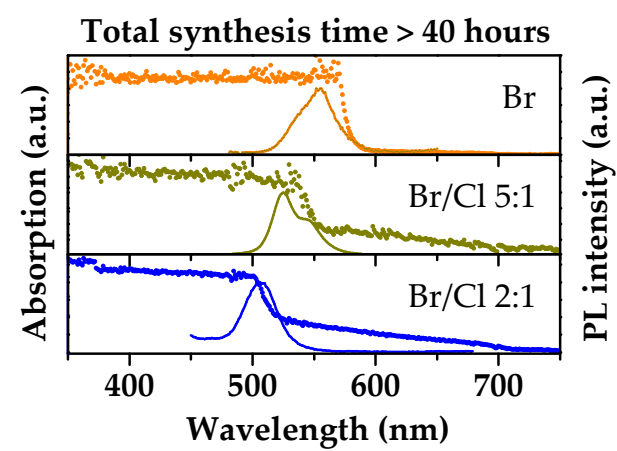

(b)

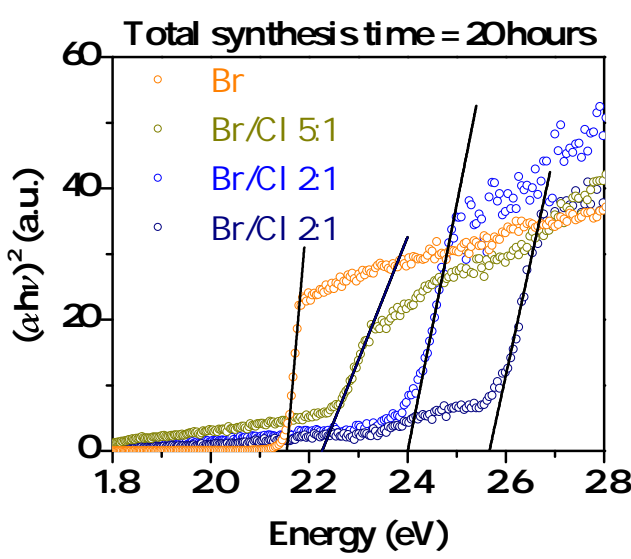

(d)

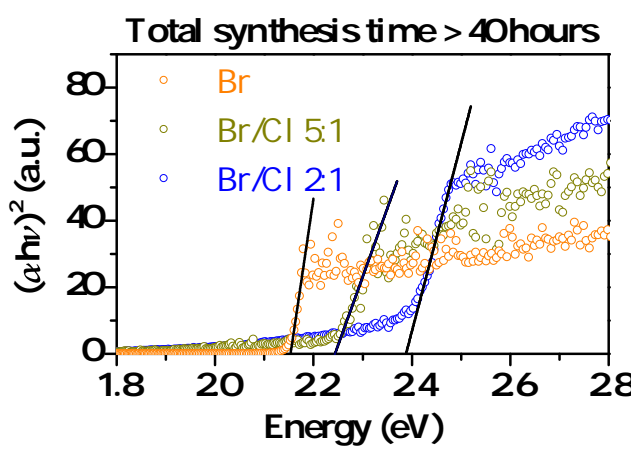

FIGURE 4.14: (a) UV-vis absorption and PL emission. (b) Tauc plots of $\mathrm{FAPbBr}_{3-\mathrm{x}} \mathrm{Cl}_{\mathrm{x}}$ based perovskites with a total synthesis time of 20 hours. (c) UV-vis absorption and PL emission. (d) Tauc plots of $\mathrm{FAPbBr}_{3-\mathrm{x}} \mathrm{Cl}_{\mathrm{x}}$ based perovskites with a total synthesis time $>40$ hours.

absorption data are collected in a transmission geometry which probes the bulk sample. This observed degradation being due to the surface is consistent with the powder of the ground single crystals appearing similar regardless of the total synthesis time as depicted in Figure 4.12(c). The PL spectra for a nominal $\mathrm{Br} / \mathrm{Cl}$ ratio of 2:1 appears to become red shifted and more narrow with the increased synthesis time. The total synthesis time appears to have had the biggest impact on the samples with a nominal ratio of $1: 1$. For the sample with a total synthesis time of 60 hours no UV-vis absorption or PL spectra could be obtained. The samples also appears to be significantly more red/brown than that with a lower $\mathrm{Cl}$ inclusion.

It is possible that the formamidinium ion may be responsible for the observed trends of $\mathrm{FAPbBr}_{3-\mathrm{x}} \mathrm{Cl}_{\mathrm{x}}$ based perovskites with increased reaction time, owing to the more hygroscopic nature of $\mathrm{FABr}$ relative to $\mathrm{MABr}$. As our reactions are carried out under ambient conditions the increased reaction times of the single crystals may lead to the degradation of FABr with prolonged exposure to a high humidity environment (40-60\%). This may lead to the 
(a)

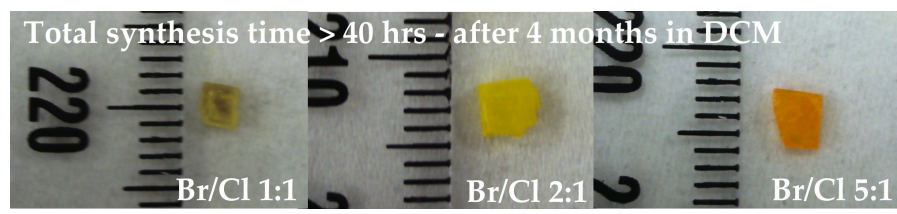

(b)

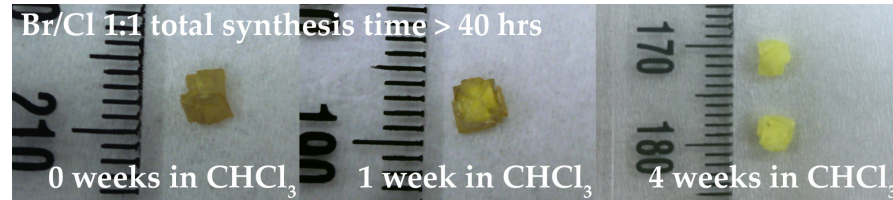

FIGURE 4.15: Surface repair of degraded $\mathrm{FAPbBr}_{3-\mathrm{x}} \mathrm{Cl}_{\mathrm{x}}$ perovskite single crystals. (a) $\mathrm{FAPbBr}_{3-\mathrm{x}} \mathrm{Cl}_{\mathrm{x}}$ single crystals with a total synthesis time $>40$ hours after 4 months in dichloromethane. (b) Nominal $\mathrm{Br} / \mathrm{Cl}$ ratio of 1:1 after 1 and 4 weeks in chloroform.

(a)

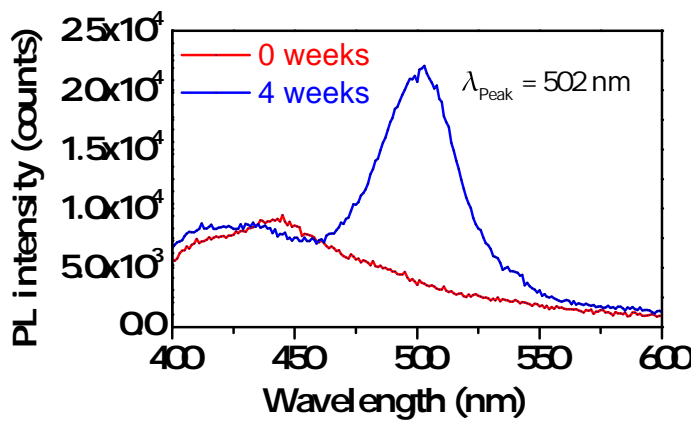

FIGURE 4.16: PL emission for degraded single crystal with a nominal $\mathrm{Br} / \mathrm{Cl}$ ratio of 1:1 before and after 4 weeks suspended in chloroform.

formation of an oxide layer or a hydrated layer on the surface of the single crystals.

Although FABr is more hygroscopic than MABr it must also be noted that the $\mathrm{FAPbBr}_{3}$ sample synthesized for $>40$ hours does not appear to have any significant differences relative to the sample synthesized for 20 hours. This suggests that the inclusion of $\mathrm{Cl}$ may also play a part in the degradation of the surface of the perovskites. The $\mathrm{MAPbBr}_{3-\mathrm{x}} \mathrm{Cl}_{\mathrm{x}}$ based perovskites do not appear to have an observed degradation of the crystal surfaces with respect to increased synthesis times. This further suggests that the presence of formamidinium and chloride are both responsible for the observed degradation of the crystal surface.

Observations of the single crystals in dichloromethane and chloroform are shown in Figure 4.15. Interestingly if we leave these degraded single crystals in a anitsolvent we observe that the crystals return to a similar appearance to those with a total synthesis time of 20 hours. It appears that the antisolvents 
(a)

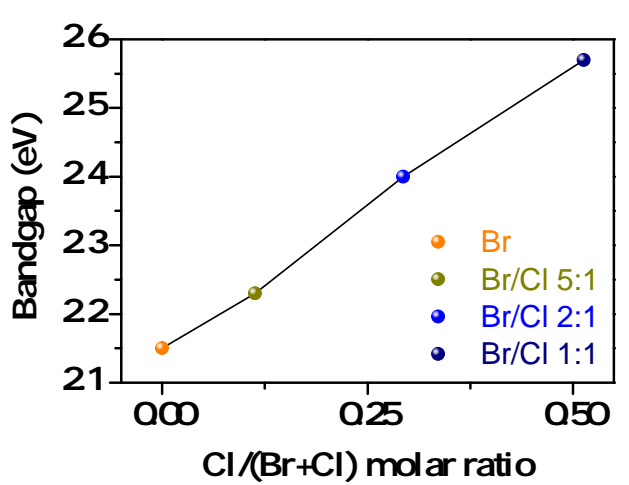

(b)

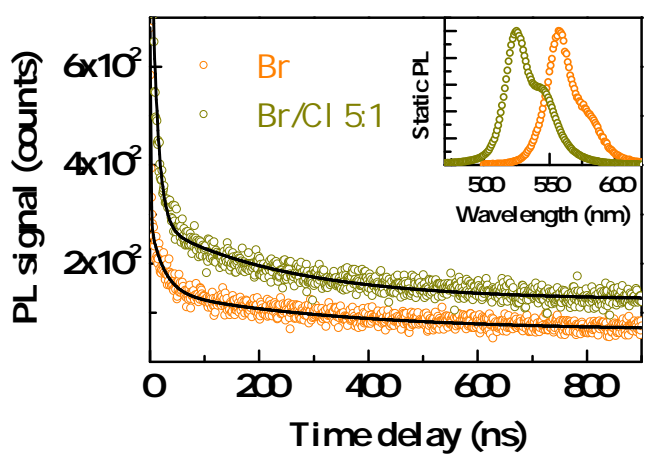

FIGURE 4.17: (a) Bandgap of $\mathrm{FAPbBr}_{3-\mathrm{x}} \mathrm{Cl}_{\mathrm{x}}$ based perovskites as a function of $\mathrm{Cl} /(\mathrm{Br}+\mathrm{Cl})$ molar ratio determined from SEM/EDS.

(b) PL time decay trace on $\mathrm{FAPbBr}_{3-\mathrm{x}} \mathrm{Cl}_{\mathrm{x}}$ based perovskites. Inset: corresponding PL emission peak.

TABLE 4.6: PL decay lifetimes for $\mathrm{FAPbBr}_{3-\mathrm{x}} \mathrm{Cl}_{\mathrm{x}}$ based perovskites.

\begin{tabular}{lcc}
\hline \multirow{2}{*}{ Nominal ratios } & \multicolumn{2}{c}{ PL decay lifetimes (ns) } \\
\cline { 2 - 3 } & $\boldsymbol{\tau}_{\mathbf{1}}$ & $\boldsymbol{\tau}_{\mathbf{2}}$ \\
\hline Pure $\mathrm{Br}$ & $24 \pm 2$ & $333 \pm 22$ \\
$\mathrm{Br} / \mathrm{Cl} 5: 1$ & $11 \pm 1$ & $246 \pm 9$ \\
\hline
\end{tabular}

are repairing the surface of the single crystals. This may suggest that the degraded surfaces are due to a hydrated chloride species where the antisolvents are removing the hydrated species from the surface of the crystal and reversing the degradation of the surface. It is also possible that the antisolvents are etching the surface of the single crystals and removing the degraded species. The increased polarity of chloroform appears to also increase the rate at which the degraded surface species are removed relative to dichloromethane. This suggests that we may be able to reverse any degradation of $\mathrm{FAPbBr}_{3-\mathrm{x}} \mathrm{Cl}_{\mathrm{x}}$ single crystals by suspending them in a strong antisolvent.

The PL emission for the sample with a nominal ratio of $\mathrm{Br} / \mathrm{Cl} 1: 1$ was obtained before and after being in chloroform for 4 weeks and is shown in Figure 4.16. We can see that the removal of the hydrated species from the surface of the single crystal using chloroform has resulted in a PL emission being observed at $502 \mathrm{~nm}$. This further confirms that the degradation of the single crystals is limited to the surface and is indeed reversible using an antisolvent treatment.

The band gap as a function of $\mathrm{Cl}$ inclusion is shown in Figure 4.17(a). The bandgap was observed to follow a linear trend with increasing $\mathrm{Cl}$ content. This shows how successfully altering the percentage of $\mathrm{Cl}$ present can be 
used in tuning specific band gaps. The PL decay curves for $\mathrm{FAPbBr}_{3-\mathrm{x}} \mathrm{Cl}_{\mathrm{x}}$ are shown in Figure 4.17(b). Due to the weak emissions for the samples with a nominal $\mathrm{Br} / \mathrm{Cl}$ ratio of 2:1 and 1:1, no PL decay curves could be measured using time correlated single photon counting. Similar to the $\mathrm{MAPbBr}_{3-\mathrm{x}} \mathrm{Cl}_{\mathrm{x}}$ based perovskites we fitted biexponential decay curves and the extracted time constants are summarized in Table 4.6. We can see that we have similar a fast surface component and slow bulk component with lifetimes comparable to those for single crystals of $\mathrm{MAPbBr}_{3}{ }^{81,82}$. As per the $\mathrm{MAPbBr}_{3-\mathrm{x}} \mathrm{Cl}_{\mathrm{x}}$ perovskites we observe a decrease in the decay life times for the mixed $\mathrm{Br} / \mathrm{Cl}$ sample, which may suggest that we have increased number of traps with increasing $\mathrm{Cl}$ inclusion.

\subsubsection{Electrical characterisation}

(a)

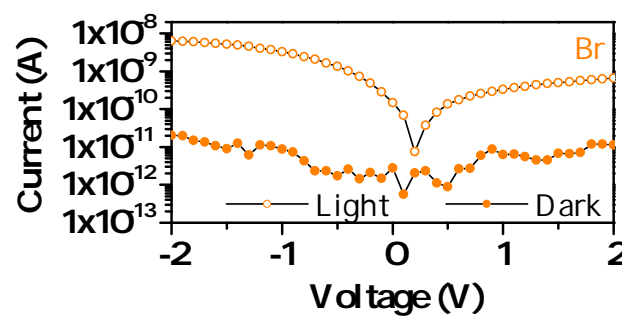

(c)

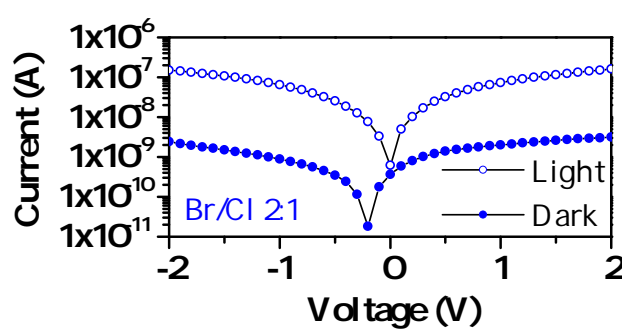

(b)

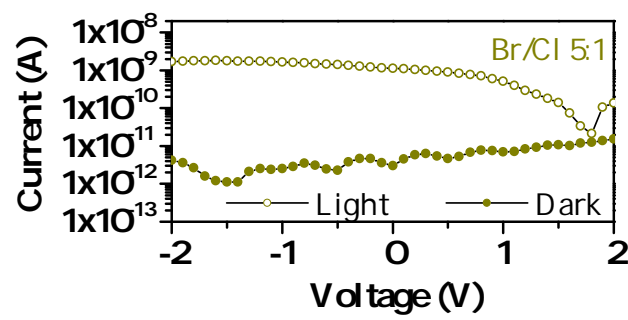

(d)

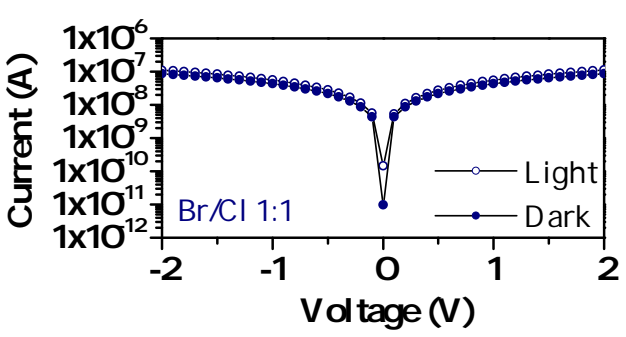

FIGURE 4.18: IV trace for (a) $\mathrm{FAPbBr}_{3}$, (b) nominal $\mathrm{Br} / \mathrm{Cl}$ 5:1, (c) nominal $\mathrm{Br} / \mathrm{Cl} 2: 1$, and (d) nominal $\mathrm{Br} / \mathrm{Cl}$ 1:1 single crystals under white light illumination of $45 \mathrm{~W} \mathrm{~m}^{-2}$ and in a dark environment.

TABLE 4.7: On/Off ratio for $\mathrm{FAPbBr}_{3-\mathrm{x}} \mathrm{Cl}_{\mathrm{x}}$ based perovskites at a $2 \mathrm{~V}$ bias under white light illumination of $45 \mathrm{~W} \mathrm{~m}^{-2}$.

\begin{tabular}{lc}
\hline Nominal ratio & On/Off ratio \\
\hline $\mathrm{Br}$ & 220 \\
$\mathrm{Br} / \mathrm{Cl} 5: 1$ & 44 \\
$\mathrm{Br} / \mathrm{Cl} 2: 1$ & 110 \\
$\mathrm{Br} / \mathrm{Cl} 1: 1$ & 1.7 \\
\hline
\end{tabular}



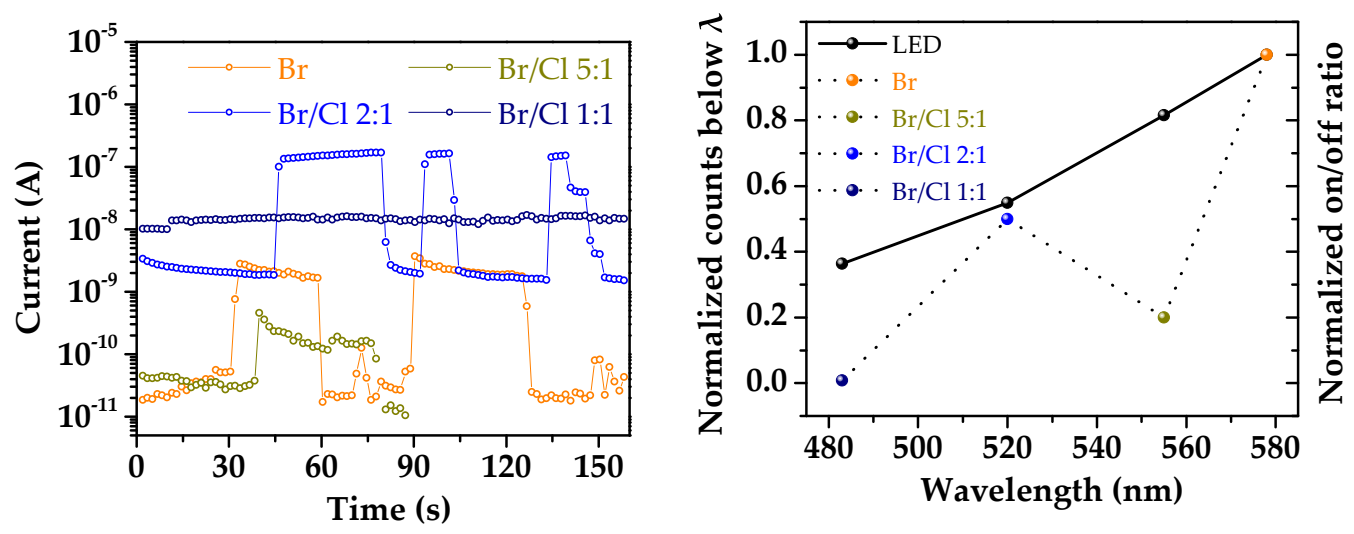

FIGURE 4.19: (a) On/off current at a 2 V bias under white light illumination of $45 \mathrm{~W} \mathrm{~m}^{-2}$ and (b) comparison of on/off ratio with white light $\mathrm{LED}$ emission spectrum for $\mathrm{FAPbBr}_{3-\mathrm{x}} \mathrm{Cl}_{\mathrm{x}}$ based perovskites.

IV characteristics are investigated using the same planar electrode geometry shown in Figure 4.7. The IV traces are shown in Figure 4.18 for $\mathrm{FAPbBr}_{3-\mathrm{x}} \mathrm{Cl}_{\mathrm{x}}$ based perovskites. In order to prepare crystals large enough to deposit electrode onto, we had to synthesis them for $>40$ hours which typically results in a surface degradation of the perovskites. Some anomalous trends are observed in the IV traces and data was only collected up to $2 \mathrm{~V}$ due to problems with contacting the electrodes and problems that may arise from the surface oxidation/hydrated species contaminating the surface of the perovskites. Nevertheless we found on/off ratio for our $\mathrm{FAPbBr}_{3-\mathrm{x}} \mathrm{Cl}_{\mathrm{x}}$ based perovskites which are summarized in Table 4.7 and shown in Figure 4.19(a). The on/off ratio are reasonable good for the pure Br sample and nominal $\mathrm{Br} / \mathrm{Cl}$ ratio of 2:1. The sample with a nominal $\mathrm{Br} / \mathrm{Cl}$ ratio 1:1 most likely suffers from the degradation of the surface, thus showing poor absorption and on/off characteristics. Comparison of the on/off ratio with the white light emission of the LED are shown in Figure 4.19. We can see that the pure Br and nominal $\mathrm{Br} / \mathrm{Cl}$ ratio of 2:1 agree reasonable well with the actual emission from the LED. These results prove inconclusive as it is unknown how the surface contamination due to hydrated or oxidised species may affect the electrical properties. 


\section{4 $\mathrm{MAPb}_{1-\mathrm{x}} \mathrm{Sn}_{\mathrm{x}} \mathrm{Br}_{3}$ based perovskites}

\subsubsection{Synthesis}

The same general ITC method and crystal washing technique for $\mathrm{MAPbBr}_{3}$ was used to grow the following perovskites with these alterations. As far as I know this is the first reported case of mixed $\mathrm{Pb} / \mathrm{Sn}$ perovskites grown using ITC.

Synthesis of $\mathrm{MAPb}_{1-\mathrm{x}} \mathrm{Sn}_{\mathrm{x}} \mathrm{Br}_{3}$ (nominal $\mathrm{x}=\mathbf{0 . 2 5 )}$ ). A $1 \mathrm{M}$ growth solution of $\mathrm{MAPb}_{0.75} \mathrm{Sn}_{0.25} \mathrm{Br}_{3}$ was prepared by dissolving $\mathrm{MABr} / \mathrm{PbBr}_{2} / \mathrm{SnBr}_{2}$ in 4:3:1 molar ratio in DMF. The crystals were then grown at $75^{\circ} \mathrm{C}$ for 1 hour under ambient conditions. The temperature was than increased to $80{ }^{\circ} \mathrm{C}$ for a further 4 hours. The growth solution was replaced 2 times for a total synthesis time of 15 hours. The single crystals were then washed and stored in DCM

Synthesis of $\mathrm{MAPb}_{1-\mathrm{x}} \mathrm{Sn}_{\mathrm{x}} \mathrm{Br}_{3}$ (nominal $\mathrm{x}=0.5$ ). A $1 \mathrm{M}$ growth solution of $\mathrm{MAPb}_{0.5} \mathrm{Sn}_{0.5} \mathrm{Br}_{3}$ was prepared in a glove box under a $\mathrm{N}_{2}$ atmosphere by dissolving $\mathrm{MABr} / \mathrm{PbBr}_{2} / \mathrm{SnBr}_{2}$ in 2:1:1 molar ratio in anhydrous DMF. The solution was then transferred onto a Schlenk line and heated to $75^{\circ} \mathrm{C}$ under $\mathrm{N}_{2}$. The temperature of the oil bath was then increased by $5{ }^{\circ} \mathrm{C}$ every hour until $85^{\circ} \mathrm{C}$ upon which the solution is left for a further 3 hours. The single crystals were then washed and stored in either DCM or in a glove box.

\subsubsection{Results and discussion}

In order to incorporate $\mathrm{Sn}$ into the metal site for MA based perovskites an equal molar ratio of $\mathrm{PbBr}_{2}$ was substituted for $\mathrm{SnBr}_{2}$. In the case of a nominal $\mathrm{Pb} / \mathrm{Sn}$ precursor ratio of 3:1 it was observed that large single crystals could be grown under ambient conditions using ITC at $75^{\circ} \mathrm{C}$. However, if a nominal $\mathrm{Pb} / \mathrm{Sn}$ precursor ratio of 1:1 was used no single crystals were observed to form using ITC under ambient conditions. Additionally, the solution was observed to transition from a colourless solution to slightly yellow colour with increasing reaction time. This is attributed to the oxidation of $\mathrm{Sn}$ (II) to Sn(IV) which appears to suppress the ITC of large single crystals. We found that large single crystals with a nominal $\mathrm{Pb} / \mathrm{Sn}$ ratio of $1: 1$ could be achieved by using ITC under an inert atmosphere. Under a $\mathrm{N}_{2}$ atmosphere large single crystals were observed to form in a matter of hours. Additionally the solution remained colourless. This confirms that the formation of Sn(IV) whether by overconsumption of the Sn(II) precursor or otherwise does appear to suppress the growth of large single crystals grown via ITC. 
TABLE 4.8: $\mathrm{Pb} / \mathrm{Sn}$ ratio determined from SEM/EDS, lattice constant determined from powder XRD, and band gap calculated from Tauc plots for $\mathrm{MAPb}_{1-\mathrm{x}} \mathrm{Sn}_{\mathrm{x}} \mathrm{Br}_{3}$ based perovskites.

\begin{tabular}{lccc}
\hline Nominal ratio & EDS ratio & Lattice constant $(\AA)$ & Band gap (eV) \\
\hline Pure $\mathrm{Br}$ & $\mathrm{N} / \mathrm{A}$ & 5.96 & $2.18\left(2.15^{*}\right)$ \\
$\mathrm{Pb} / \mathrm{Sn} 3: 1$ & $\mathrm{~Pb} / \mathrm{Sn} 99: 1$ & 5.96 & $1.83\left(1.74^{*}\right)$ \\
$\mathrm{Pb} / \mathrm{Sn} 1: 1$ & $\mathrm{~Pb} / \mathrm{Sn} 32: 1$ & 5.97 & $2.04\left(1.69^{*}\right)$ \\
\hline
\end{tabular}

${ }^{*}$ Due to the highly scattering nature of Sn doped samples a scattering independent UV-vis method was also used to measure the absorption. The band gap determined from this approach are given in the parenthesis.

Photographs of the $\mathrm{MAPb}_{1-\mathrm{x}} \mathrm{Sn}_{\mathrm{x}} \mathrm{Br}_{3}$ based perovskites are shown in Figure 4.20(a). We can see that with increasing Sn inclusion the single crystals appear to shift from orange to red and finally to dark red for nominal precursor ratios of pure $\mathrm{Br}, \mathrm{Pb} / \mathrm{Sn}$ 3:1 and $\mathrm{Pb} / \mathrm{Sn}$ 1:1 respectively. Powder XRD spectra are shown in Figure 4.20(b). The powder XRD spectra along with the lattice constants summarized in Table 4.8 show negligible changes indicating very little $\mathrm{Sn}$ was incorporated into the single crystal perovskites.

Elemental composition of $\mathrm{MAPb}_{1-\mathrm{x}} \mathrm{Sn}_{\mathrm{x}} \mathrm{Br}_{3}$ single crystals are summarized in Table 4.8 and show a $1 \%$ and $3 \%$ inclusion of Sn for a nominal precursor ratio of $\mathrm{Pb} / \mathrm{Sn}$ 3:1 and 1:1 respectively. This shows a significant preferential inclusion of $\mathrm{Pb}$ over $\mathrm{Sn}$. It should be noted that it is unknown whether the incorporated $\mathrm{Sn}$ is $\mathrm{Sn}(\mathrm{II})$ or $\mathrm{Sn}(\mathrm{IV})$. SEM-SEI images of the crystal surface are shown in Figure 4.21. The surface appears to be rough with perhaps an increase in the roughness with increasing Sn inclusion.

(a)

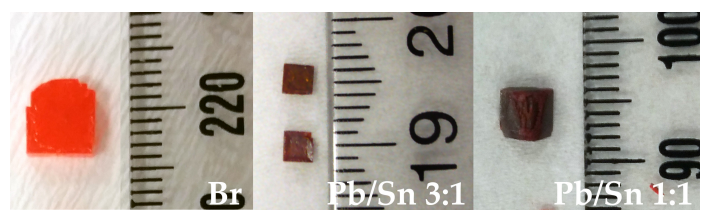

(b)

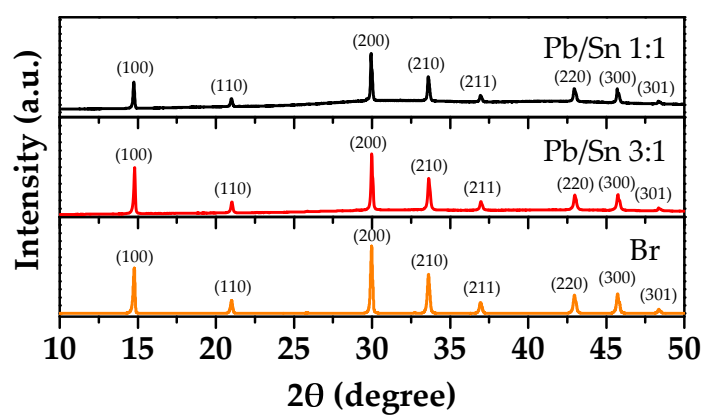

FIGURE 4.20: (a) Photographs and (b) powder XRD spectra of $\mathrm{MAPb}_{1-\mathrm{x}} \mathrm{Sn}_{\mathrm{x}} \mathrm{Br}_{3}$ based perovskites. 
(a)

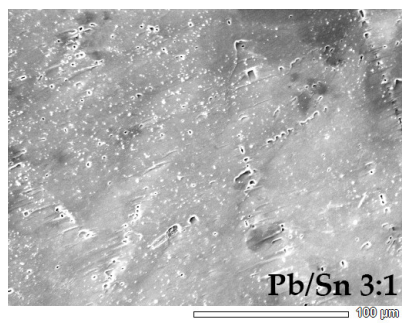

(b)

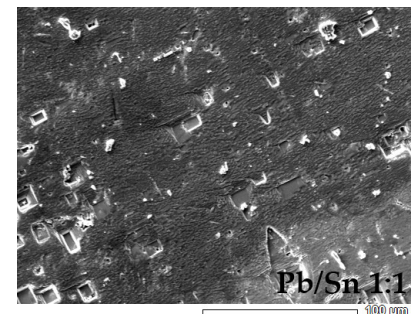

(c)

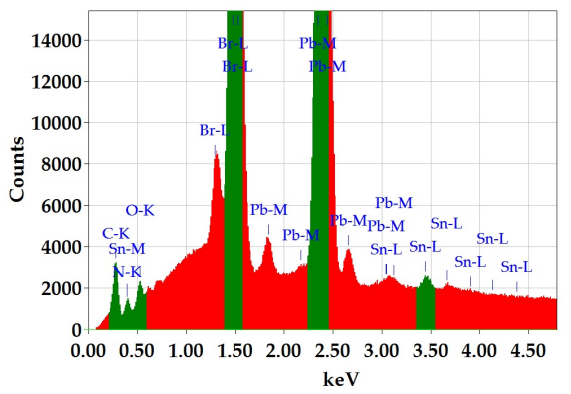

FIGURE 4.21: SEM-SEI images of $\mathrm{MAPb}_{1-\mathrm{x}} \mathrm{Sn}_{\mathrm{x}} \mathrm{Br}_{3}$ perovskites.

(a) nominal $\mathrm{Pb} / \mathrm{Sn}$ ratio of 3:1 (b) nominal $\mathrm{Pb} / \mathrm{Sn}$ ratio of $1: 1$

(c) EDS spectrum for nominal $\mathrm{Pb} / \mathrm{Sn}$ ratio of 3:1 showing the presence of a Sn peak.

(a)

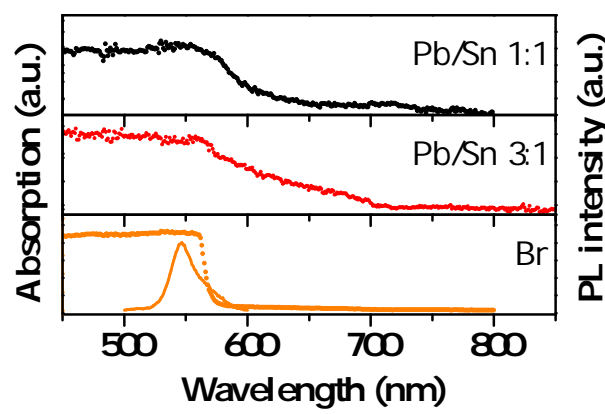

(c)

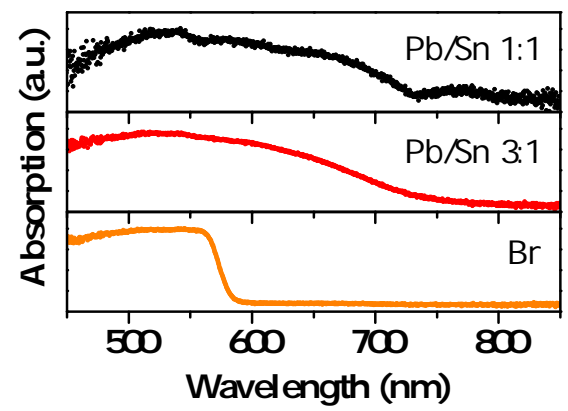

(b)

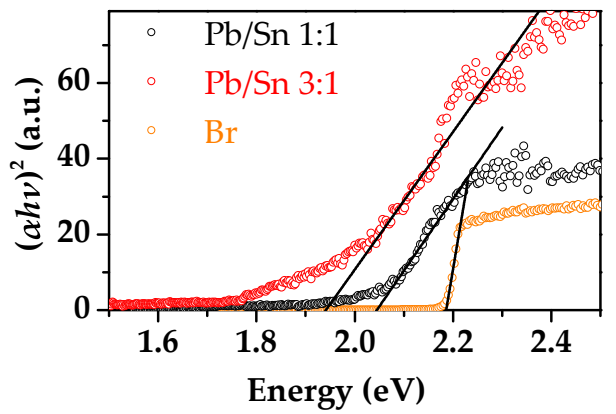

(d)

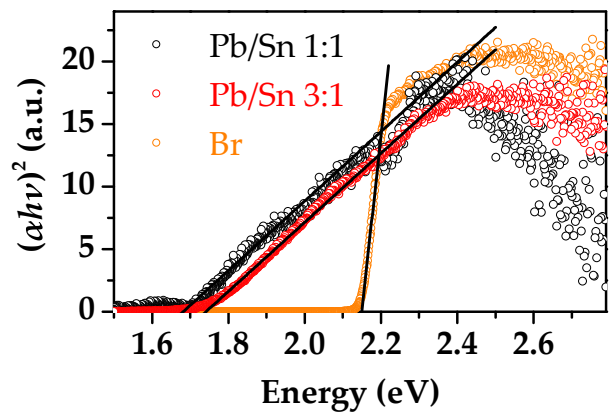

FIGURE 4.22: (a) UV-vis absorption and corresponding (b) Tauc plots of $\mathrm{MAPb}_{1-\mathrm{x}} \mathrm{Sn}_{\mathrm{x}} \mathrm{Br}_{3}$ based perovskites. (c) Scattering independent UV-vis absorption and corresponding (d) Tauc plots of $\mathrm{MAPb}_{1-\mathrm{x}} \mathrm{Sn}_{\mathrm{x}} \mathrm{Br}_{3}$ based perovskites.

The absorption spectra and corresponding Tauc plots of $\mathrm{MAPb}_{1-\mathrm{x}} \mathrm{Sn}_{\mathrm{x}} \mathrm{Br}_{3}$ based perovskites are shown in Figure 4.22. We can see that the inclusion of even $1 \%$ of $\mathrm{Sn}$ results in a much broader absorption edge. This broadening 


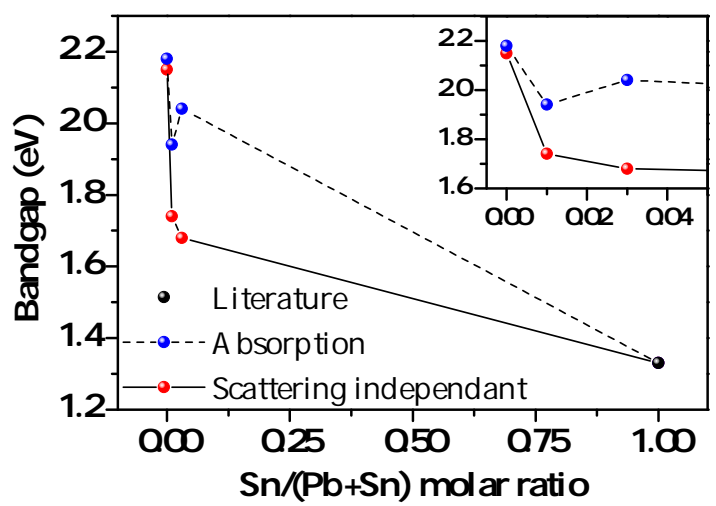

FIGURE 4.23: Bandgap of $\mathrm{MAPb}_{1-\mathrm{x}} \mathrm{Sn}_{\mathrm{x}} \mathrm{Br}_{3}$ as a function of $\mathrm{Sn} /(\mathrm{Pb}+\mathrm{Sn})$ molar ratio determined from SEM/EDS. The bandgap for pure $\mathrm{MAPbSn}_{3}$ was reported by Mancini et al. $(2015)^{102}$. Inset: Shows the bandgap with a molar ratio in the region of $0.00-0.05$.

TABLE 4.9: On/Off ratio for $\mathrm{MAPb}_{1-\mathrm{x}} \mathrm{Sn}_{\mathrm{x}} \mathrm{Br}_{3}$ based perovskites at a $2 \mathrm{~V}$ bias under white light illumination of $45 \mathrm{~W} \mathrm{~m}^{-2}$.

\begin{tabular}{lc}
\hline Nominal ratio & On/Off ratio \\
\hline $\mathrm{Br}$ & 1200 \\
$\mathrm{~Pb} / \mathrm{Sn} 3: 1$ & 72 \\
$\mathrm{~Pb} / \mathrm{Sn} 1: 1$ & 13 \\
\hline
\end{tabular}

of the absorption maybe in part due to scattering. No PL emissions were observed which may be related to the weaker absorptions observed. Scattering independent absorption spectra were also collected using a setup provided by the Le Ru group at Victoria University of Wellington ${ }^{101}$. In this setup the influence of scattering is eliminated with a direct measurement of absorbance, rather than extinction. The scattering independent absorption spectra are shown in Figure 4.22(c) along with the corresponding Tauc plots. This shows a similar trend to the absorption spectra in which we see a broad absorption edge which is red shifted with increasing Sn incorporation which is consistent with the observed colours as depicted in Figure 4.20(a).

The extrapolated band gaps are summarized in Table 4.8 and shown in Figure 4.23. The bandgap for the Sn doped perovskites all appear to be red shifted and lower than the pure $\mathrm{MAPbBr} 3$ based perovskite. There does not appear to be an linear trend for the band gap. Even with a small percent of Sn incorporated we can see significant changes to the band gap. This is contrary to solid solutions of $\mathrm{MAPb}_{1-\mathrm{x}} \mathrm{Sn}_{\mathrm{x}} \mathrm{Br}_{3}$ based perovskites which show a bandgap of $2.00 \mathrm{eV}$ and $1.75 \mathrm{eV}$ for $9 \%$ and $65 \%$ of incorporated $\mathrm{Sn}$ respectively ${ }^{102}$.

The IV characteristics are investigated using the same planar electrode 
(a)

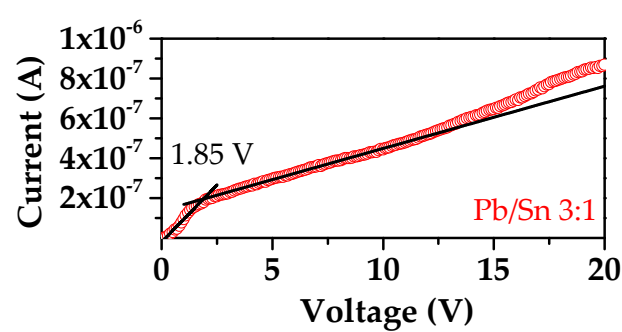

(c)

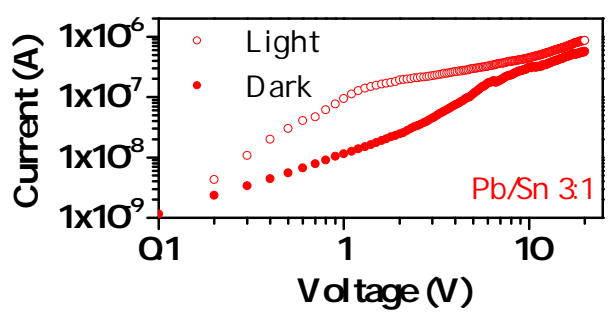

(b)

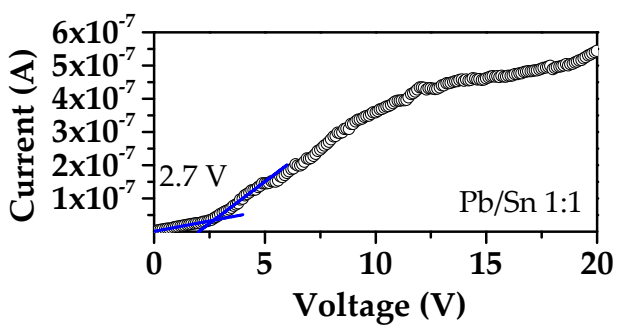

(d)

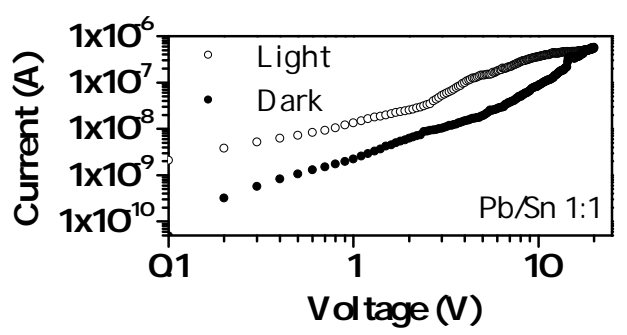

FIGURE 4.24: (a)(b) IV trace for $\mathrm{MAPb}_{1-\mathrm{x}} \mathrm{Sn}_{\mathrm{x}} \mathrm{Br}_{3}$ under white light illumination of $45 \mathrm{~W} \mathrm{~m}^{-2}$. (c)(d) IV trace of $\mathrm{MAPb}_{1-x} \mathrm{Sn}_{\mathrm{x}} \mathrm{Br}_{3}$ under white light illumination of $45 \mathrm{~W} \mathrm{~m}^{-2}$ and in a dark environment.

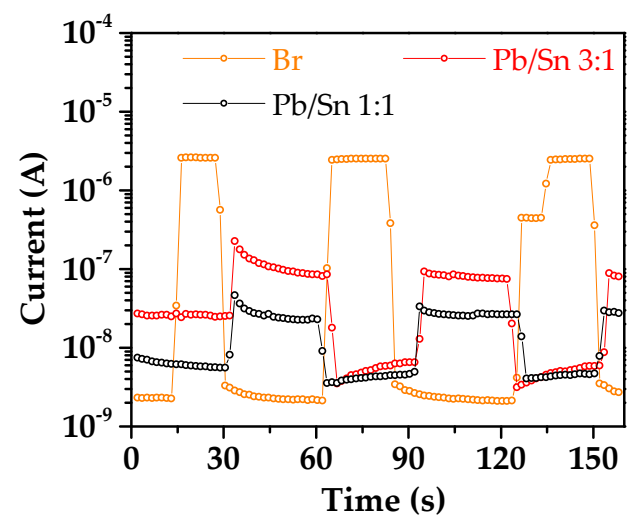

FIGURE 4.25: On/off current for $\mathrm{MAPb}_{1-\mathrm{x}} \mathrm{Sn}_{\mathrm{x}} \mathrm{Br}_{3}$ based perovskites at $2 \mathrm{~V}$ bias under white light illumination of $45 \mathrm{~W} \mathrm{~m}^{-2}$.

geometry shown in Figure 4.7. IV traces under white light illumination of $45 \mathrm{~W} \mathrm{~m}^{-2}$ and in a dark environment are shown in Figure 4.24. Using the tentative model proposed for $\mathrm{MAPbBr}_{3}$ we obtain two linear regions for the sample doped with $1 \%$ Sn which are shown in Figure 4.24(a). In the case of the sample with 3\% Sn the model does not seem to be valid as section at low voltages appears to have lower mobility than the high voltage region. This may be due to the reduced absorption with increasing $S n$ inclusion. Although PL lifetimes could not be found for the Sn based perovskites due to weak emissions the diffusion length for the $1 \%$ sample was estimated to be 1-14 $\mu \mathrm{m}$. This is comparable to the diffusion lengths found for pure $\mathrm{Br}$ of $2-8 \mu \mathrm{m}$. 
The on/off ratio for $\mathrm{MAPb}_{1-\mathrm{x}} \mathrm{Sn}_{\mathrm{x}} \mathrm{Br}_{3}$ based perovskites are shown in Figure 4.25 and summarized in Table 4.9. We can see that the on/off ratio is significantly lower than the pure Br material which is most likely due to the much weaker absorptions observed with increasing Sn inclusion.

\subsection{Thermoelectric properties}

We attempted to measure the thermoelectric properties of a single crystal of $\mathrm{MAPbBr}_{3}$ using the PPMS. However several problems arose. The first problem was that the single crystals appeared to self cleave during the cooling phase and no data could be collected. This was remedied by starting the measurements at room temperature first. However it was also found that the resistance of the single crystal was $>20 \mathrm{M} \Omega$ and hence no resistivity or thermopower data could be measured. The thermal conductivity was measured using the PPMS and is shown in Figure 4.26 along with the single crystal mounted for thermoelectric measurements. The thermal conductivity at room temperature was $\sim 1.12 \mathrm{~W} \mathrm{~m}^{-1} \mathrm{~K}^{-1}$ which is relatively low for single crystals and is on par with the thermal conductivity needed to produce a high $Z T$ as predicted by computational calculations ${ }^{14}$. However it is not as low as the thermal conductivity of $\sim 0.6 \mathrm{~W} \mathrm{~m}^{-1} \mathrm{~K}^{-1}$ reported for $\mathrm{MAPbI}_{3}$ single crystals ${ }^{63}$. Although the thermoelectric properties could not be measured due to the high resistance of our single crystals we were prompted to try to incorporate mixed halides and mixed metal sites into our single crystal perovskites in order to decrease the resistance. This ultimately lead to single crystals with interesting optical and electrical properties, however the problems with self cleaving and a high resistance under a dark environment made measuring the thermoelectric properties challenging.

(a)

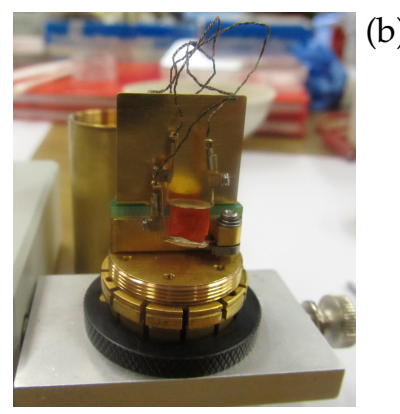

(b)

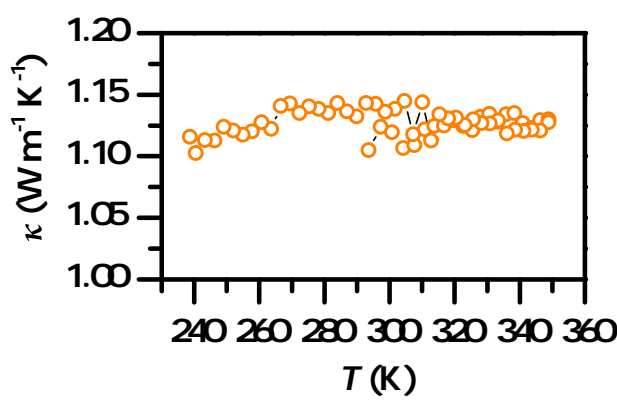

FIGURE 4.26: (a) $\mathrm{MAPbBr}_{3}$ mounted for thermoelectric measurements using the PPMS (b) thermal conductivity of $\mathrm{MAPbBr}_{3}$ single crystal. 
TABLE 4.10: Dark conductance for MA based perovskites.

\begin{tabular}{lc}
\hline Nominal ratio & Conductance $\left(\Omega^{-1}\right)$ \\
\hline $\mathrm{Br}$ & $6.1 \times 10^{-10}$ \\
$\mathrm{Br} / \mathrm{Cl} 5: 1$ & $9.5 \times 10^{-10}$ \\
$\mathrm{Br} / \mathrm{Cl} 5: 1$ & $1.9 \times 10^{-10}$ \\
$\mathrm{Cl}$ & $1.3 \times 10^{-10}$ \\
$\mathrm{~Pb} / \mathrm{Sn} 3: 1$ & $2.8 \times 10^{-8}$ \\
$\mathrm{~Pb} / \mathrm{Sn} 1: 1$ & $5.3 \times 10^{-8}$ \\
\hline
\end{tabular}

The conductance of the single crystal perovskites under a dark environment are found by fitting a linear trends in the voltage range of $10-20 \mathrm{~V}$ for the IV traces shown in Figure 4.9 and Figure 4.23. The conductances are summarized in Table 4.10. The mixed $\mathrm{Br} / \mathrm{Cl}$ samples all appear to have similar conductances with no discernible trend with increasing chloride content. This suggests that the conductivity of our mixed $\mathrm{Br} / \mathrm{Cl}$ perovskites has not changed significantly. However the $\mathrm{MAPb}_{1-\mathrm{x}} \mathrm{Sn}_{\mathrm{x}} \mathrm{Br}_{3}$ based perovskites have conductances 2 order of magnitudes higher than the $\mathrm{MAPbBr}_{3-\mathrm{x}} \mathrm{Cl}_{\mathrm{x}}$ based perovskites. This suggests that mixed metals of $\mathrm{Pb} / \mathrm{Sn}$ may lead to significant improvements in the electrical conductivity and figure of merit of single crystal perovksite thermoelectric materials.

\subsection{Summary}

Through optimising the polarity of the solvent using DMF/DMSO and the crystallisation temperature large millimetre scale single crystals of $\mathrm{MAPbBr}_{3-\mathrm{x}} \mathrm{Cl}_{\mathrm{x}}$ were synthesized using ITC in a matter of days. It was found that the bandgap could be tuned between $2.18 \mathrm{eV}$ and $2.88 \mathrm{eV}$ by altering the $\mathrm{Br} / \mathrm{Cl}$ ratio. The mobility and diffusion length for some single crystals were estimated using a tentative model. The mobilities and diffusion lengths were estimated to be $\mu \approx 30-390 \mathrm{~cm}^{2} \mathrm{~V}^{-1} \mathrm{~s}^{-1}$ and $L_{D} \approx 2-8 \mu \mathrm{m}$ for pure $\mathrm{Br}$ and $\mu \approx 10-100$ $\mathrm{cm}^{2} \mathrm{~V}^{-1} \mathrm{~s}^{-1}$ and $L_{D} \approx 1-4 \mu \mathrm{m}$ for a nominal $\mathrm{Br} / \mathrm{Cl}$ ratio of 5:1.

Through optimising the concentration and crystallisation temperature large single crystal based perovskites of $\mathrm{FAPbBr}_{3-\mathrm{x}} \mathrm{Cl}_{\mathrm{x}}$ are synthesized. As far as I am aware this is the first reported case of mixed $\mathrm{Br} / \mathrm{Cl}$ single crystals with a FA cation synthesized using ITC. It was observed that with increasing the reaction time and increasing $\mathrm{Cl}$ inclusion the surface of the single crystal became degraded. It was found that suspending the degraded single crystals in a antisolvent such as chloroform could remove the degraded species from 
the surface. The bandgap of $\mathrm{FAPbBr}_{3-\mathrm{x}} \mathrm{Cl}_{\mathrm{x}}$ based perovskites was found to be tunable between $2.15 \mathrm{eV}$ and $2.57 \mathrm{eV}$ by altering the $\mathrm{Br} / \mathrm{Cl}$ ratio. Unfortunately the electrical characterisation did not present any conclusive data owing to the surface degradation of the single crystals. Further experiments must be carried out on the cleaned species in order to elucidate their electrical properties.

$\mathrm{MAPb}_{1-\mathrm{x}} \mathrm{Sn}_{\mathrm{x}} \mathrm{Br}_{3}$ based perovskites were successfully synthesized using ITC. As far as I am aware this is the first reported case of Sn inclusion into large single crystals grown by ITC. It was found that the inclusion of even $1 \%$ of $\mathrm{Sn}$ resulted in a significant broadening and red shift of the absorption edge. Unfortunately no mobilities could be determined due to the weak PL emissions after Sn incorporation. However using our tentative model the diffusion length was found to be $1-14 \mu \mathrm{m}$ for samples with $1 \%$ of $\mathrm{Sn}$ incorporated.

The thermoelectric properties of perovskite single crystals could not be measured due to the high resistance of the single crystal and self cleaving nature of the single crystals with decreasing temperature. Nevertheless the thermal conductivity was found to be $\sim 1.12 \mathrm{~W} \mathrm{~m}^{-1} \mathrm{~K}^{-1}$ at room temperature for $\mathrm{MAPbBr}_{3}$ which is reasonable low. It was also found that the conductance of the single crystals increased by 2 orders of magnitude with the inclusion of Sn. 


\section{Chapter 5}

\section{Conclusion and future work}

\subsection{Conclusion}

In conclusion we have synthesized nanostructured $\mathrm{Bi}_{2} \mathrm{Te}_{3}$ using a bismuth(III) oleate precursor. The nanoparticles produced from this approach were found to be extremely agglomerated particles consisting of thin nanoplates with a wide size distribution. The effect of different capping ligands such as OLA, DDT and TGA were found to have minimal effect on the morphology of solution processed nanoparticles. The nanostructured $\mathrm{Bi}_{2} \mathrm{Te}_{3}$ produced using this approach was found to have exceptionally low thermal conductivities of $\sim$ $0.11 \mathrm{~W} \mathrm{~m}^{-1} \mathrm{~K}^{-1}$, which is significantly lower than the bulk and nanostructured $\mathrm{Bi}_{2} \mathrm{Te}_{3}$ which has a optimal thermal conductivity of $\sim 0.8 \mathrm{~W} \mathrm{~m}^{-1} \mathrm{~K}^{-1} \quad 2,33,40$. However due to the poor electrical conductivity the overall figure of merit for these materials was only $\sim 4 \times 10^{-6}$ at room temperature which is significantly lower than the figure of merit of 0.18 reported by Scheele et al. for $\mathrm{Bi}_{2} \mathrm{Te}_{3}$ nanoparticles ${ }^{40}$.

Using a Bi nanoparticles precursor aggregated sub- $15 \mathrm{~nm}$ spherical nanoparticles were synthesized at $60^{\circ} \mathrm{C}$ and sub- $100 \mathrm{~nm}$ rhomboid shaped particles were produced at $120^{\circ} \mathrm{C}$. In the case of $\mathrm{Bi}_{2} \mathrm{Te}_{3}$ synthesized at $120^{\circ} \mathrm{C}$ it was found that ligand exchange with hydrazine could lead to a significant increase in the figure of merit due to a doubling of the electrical conductivity while the thermal conductivity and thermopower were largely unaffected by the hydrazine treatment. After ligand exchange treatment the sample had a thermopower of $\sim 96 \mu \mathrm{V} \mathrm{K}^{-1}$ and a thermal conductivity of $\sim 0.38 \mathrm{~W} \mathrm{~m}^{-1} \mathrm{~K}^{-1}$ at $300 \mathrm{~K}$. This is an improvement over the thermopower of $\sim 60 \mu \mathrm{V} \mathrm{K}^{-1}$ and thermal conductivity of $\sim 0.8 \mathrm{~W} \mathrm{~m}^{-1} \mathrm{~K}^{-1}$ reported by Scheele et al. (2009) for $\mathrm{Bi}_{2} \mathrm{Te}_{3}$ nanoparticles ${ }^{40}$. Overall the figure of merit is still low due to the electrical conductivity which was found to be $\sim 13.1 \mathrm{~S} \mathrm{~m}^{-1}$ after hydrazine treatment. This is significantly lower than the $\sim 77000 \mathrm{~S} \mathrm{~m}^{-1}$ for $\mathrm{Bi}_{2} \mathrm{Te}_{3}$ nanoparticles ${ }^{40}$. 
$\mathrm{Cu}, \mathrm{Ni}$ and $\mathrm{Co}$ were successfully incorporated into $\mathrm{Bi}_{2} \mathrm{Te}_{3}$ using the bismuth nanoparticle precursor approach The addition of copper acetylacetonate was found to greatly affect the morphology of bismuth precursor and resulting $\mathrm{Bi}_{2} \mathrm{Te}_{3}$ nanoparticles. The bismuth nanoparticles were found to increase in size from $41 \mathrm{~nm}$ to $90 \mathrm{~nm}$ in the presence of copper acetylacetonate. The resulting $\mathrm{Bi}_{2} \mathrm{Te}_{3}$ nanoparticles were highly monodisperse with an average size of only $11 \mathrm{~nm}$ which is significantly smaller than the sub-100 nm particles observed for undoped $\mathrm{Bi}_{2} \mathrm{Te}_{3}$. Only $2 \%$ of copper was incorporated into the nanostructured $\mathrm{Bi}_{2} \mathrm{Te}_{3}$ using a precursor ratio of $10 \%$. In the case of $\mathrm{Ni}$ and Co $11 \%$ and $15 \%$ respectively were successfully incorporated into $\mathrm{Bi}_{2} \mathrm{Te}_{3}$ for a precursor ratio of $10 \%$. The $\mathrm{Ni}$ and $\mathrm{Co}$ alloys of $\mathrm{Bi}_{2} \mathrm{Te}_{3}$ were found to have a similar morphology to the undoped $\mathrm{Bi}_{2} \mathrm{Te}_{3}$ sample. All three doped/alloyed samples showed a decrease in the thermal conductivity relative to undoped $\mathrm{Bi}_{2} \mathrm{Te}_{3}$, achieving a thermal conductivity of $\sim 0.19 \mathrm{~W} \mathrm{~m}^{-1} \mathrm{~K}^{-1}$ for Co and $\mathrm{Ni}$ alloyed samples and $0.13 \mathrm{~W} \mathrm{~m}^{-1} \mathrm{~K}^{-1}$ for the $\mathrm{Cu}$ doped sample. In the case of $\mathrm{Ni}$ and Co alloys this is attributed to lattice anharmonicty leading to phononphonon umklapp scattering and also scattering from point defects. In the case of $\mathrm{Cu}$ doped sample the reduced thermal conductivity was due to the increased scattering arising from the increased number of interfaces present due to the smaller particle size compared to undoped $\mathrm{Bi}_{2} \mathrm{Te}_{3}$. In the case of $\mathrm{Ni}$ and $\mathrm{Cu}$ doped $\mathrm{Bi}_{2} \mathrm{Te}_{3}$ the figure of merit did not increase owing to the minimal improvements in the power factor. Further experiments must be carried out with respect to altering the dopant concentration before $\mathrm{Ni}$ and $\mathrm{Cu}$ can be completely dismissed as having minimal effect on the thermoelectric properties. In the case of Co doped $\mathrm{Bi}_{2} \mathrm{Te}_{3}$ the sample saw a $40 \%$ increase in the figure of merit owing to the improved electrical conductivity and reduced thermal conductivity. This shows the potential for using Co inclusion to increase the figure of merit for nanostructured $\mathrm{Bi}_{2} \mathrm{Te}_{3}$.

Single crystal perovskites of $\mathrm{MAPbBr}_{3-\mathrm{x}} \mathrm{Cl}_{\mathrm{x}}$ were grown using ITC. Through optimising the polarity of the solvent using DMF/DMSO and the crystallisation temperature large millimetre scale single crystals were achieved in a matter of days. The bandgap was found to be tunable between $2.18 \mathrm{eV}$ and $2.88 \mathrm{eV}$ by altering the $\mathrm{Br} / \mathrm{Cl}$ ratio. The mobility and diffusion length for single crystals were estimated using a planar electrode geometry. The mobility was found to be between $30-390 \mathrm{~cm}^{2} \mathrm{~V}^{-1} \mathrm{~s}^{-1}$ and $10-100 \mathrm{~cm}^{2} \mathrm{~V}^{-1} \mathrm{~s}^{-1}$ for $\mathrm{MAPbBr}_{3}$ and a nominal $\mathrm{Br} / \mathrm{Cl}$ ratio of 5:1. The diffusion length was also estimated to be between 2-8 $\mu \mathrm{m}$ and 1-4 $\mu \mathrm{m}$ for $\mathrm{MAPbBr}_{3}$ and a nominal $\mathrm{Br} / \mathrm{Cl}$ ratio of 5:1, which is comparable to single crystals of $\mathrm{MAPbBr}_{3}$ grown 
using VAC and ITC 81,82 .

For the first time large single crystals of $\mathrm{FAPbBr}_{3-\mathrm{x}} \mathrm{Cl}_{\mathrm{x}}$ based perovskites were grown using ITC. It was observed that with increasing reaction time and increasing $\mathrm{Cl}$ inclusion the surface of the single crystals became more degraded. This resulted in the suppression of the optical properties. It was found that suspending the degraded single crystals in a antisolvent such as chloroform could repair/remove the degraded species from the surface. The bandgap for $\mathrm{FAPbBr}_{3-\mathrm{x}} \mathrm{Cl}_{\mathrm{x}}$ based perovskites was found to be tunable between $2.15 \mathrm{eV}$ and $2.57 \mathrm{eV}$ by altering the $\mathrm{Br} / \mathrm{Cl}$ ratio. Owing to the surface degradation of the single crystals no conclusive electrical characterisation could be made. Further experiments must be carried on cleaned species in order to elucidate the electrical properties.

For the first time $\mathrm{MAPb}_{1-\mathrm{x}} \mathrm{Sn}_{\mathrm{x}} \mathrm{Br}_{3}$ based perovskites were successfully synthesized using ITC. It was found that $\mathrm{Pb}$ was preferentially included into the single crystals relative to $\mathrm{Sn}$, however it was found that even the inclusion of $1 \%$ of Sn resulted in a significant broadening and red-shift of the absorption edge. The conductance of the single crystals increased by 2 orders of magnitude with the inclusion of just $1 \% \mathrm{Sn}$. The thermal conductivity of $\mathrm{MAPbBr}_{3}$ was found to be $\sim 1.12 \mathrm{~W} \mathrm{~m}^{-1} \mathrm{~K}^{-1}$ at room temperature which is reasonably low for single crystals. This shows the potential of single crystals for applications in thermoelectric materials.

\subsection{Future work}

Further improvements to the figure of merit for the nanostructured $\mathrm{Bi}_{2} \mathrm{Te}_{3}$ may be possible by tuning the dopant concentration. Investigation of the morphology and thermoelectric effects with respect to dopant concentration of $\mathrm{Cu}, \mathrm{Ni}$ and $\mathrm{Co}$ should be carried out in order to understand any trends and observations. The use of post annealing or a hot pressing techniques on the nanocomposite pellets should also be investigated, as this expected to lead significant improvements in the figure of merit of our nanostructured materials ${ }^{75}$. Further work on increasing the yield of SnSe nanostructures must also be carried out in order to produce enough material for pellet consolidation and thermoelectric measurements.

Investigation of the electrical properties of $\mathrm{FAPbBr}_{3-\mathrm{x}} \mathrm{Cl}_{\mathrm{x}}$ should be carried out after repairing the surfaces using an antisolvent such as chloroform. A range of single crystals could also be attempted using ITC by making the appropriate precursor substitutions, such as pure $\mathrm{MASnBr}_{3}, \mathrm{CsPbBr}_{3}$, 
$\mathrm{CsPbBr}_{3-\mathrm{x}} \mathrm{Cl}_{x}, \mathrm{FAPb}_{1-\mathrm{x}} \mathrm{Sn}_{\mathrm{x}} \mathrm{Br}_{3}, \mathrm{FA}_{1-\mathrm{x}} \mathrm{Cs}_{\mathrm{x}} \mathrm{PbBr}_{3}$, and $\mathrm{MA}_{1-\mathrm{x}} \mathrm{Cs}_{\mathrm{x}} \mathrm{PbBr}_{3}$. The method of ITC may also be useful in growing single crystal arrays. This can be done by growing seed crystals of $\mathrm{PbX}_{2}$ onto prepatterned substrates, the seeded substrates can then be placed into a perovskite growth solution were heating can be used to initiate the inverse temperature crystallisation of the perovskite single crystals ${ }^{103,104}$. This would allow us to achieve single crystal perovskite arrays using a low temperature solution based processes which would lead to significant reduction in processing costs and higher throughput. 


\section{Bibliography}

1. Volz, S. \& SpringerLink. Thermal Nanosystems and Nanomaterials (SpringerVerlag Berlin Heidelberg, Berlin, Heidelberg, 2009).

2. Rowe, D. M. Thermoelectrics handbook: macro to nano-structured materials (Taylor\&Francis, Boca Raton, FL, 2006).

3. Biswas, K. et al. High-performance bulk thermoelectrics with all-scale hierarchical architectures. Nature 489, 414-418 (Sept. 2012).

4. Poudel, B. et al. High-thermoelectric performance of nanostructured bismuth antimony telluride bulk alloys. English. Science 320, 634-638. ISSN: 0036-8075 (2008).

5. Venkatasubramanian, R., Siivola, E., Colpitts, T. \& O'Quinn, B. Thin-film thermoelectric devices with high room-temperature figures of merit. Nature 413, 597-602 (Oct. 2001).

6. Joshi, G. et al. Enhanced Thermoelectric Figure-of-Merit in Nanostructured p-type Silicon Germanium Bulk Alloys. Nano Letters 8, 4670-4674 (Dec. 2008).

7. Heremans, J. P. et al. Enhancement of thermoelectric efficiency in $\mathrm{PbTe}$ by distortion of the electronic density of states. Science 321, 554-557 (July 2008).

8. Pei, Y. Z. et al. Convergence of electronic bands for high performance bulk thermoelectrics. Nature 473, 66-69 (May 2011).

9. Shi, X. et al. Multiple-Filled Skutterudites: High Thermoelectric Figure of Merit through Separately Optimizing Electrical and Thermal Transports. Journal of the American Chemical Society 133, 7837-7846 (May 2011).

10. Chung, D. Y. et al. CsBi4Te6: A high-performance thermoelectric material for low-temperature applications. Science 287, 1024-1027 (Feb. 2000).

11. Toberer, E. S., May, A. F. \& Snyder, G. J. Zintl Chemistry for Designing High Efficiency Thermoelectric Materials. Chemistry of Materials 22, 624634 (Feb. 2010). 
12. Zhao, L. D. et al. Ultralow thermal conductivity and high thermoelectric figure of merit in SnSe crystals. Nature 508, 373-+ (Apr. 2014).

13. Zhao, L. D., Chang, C., Tan, G. J. \& Kanatzidis, M. G. SnSe: a remarkable new thermoelectric material. Energy E Environmental Science 9, 30443060 (2016).

14. He, Y. P. \& Galli, G. Perovskites for Solar Thermoelectric Applications: A First Principle Study of CH3NH3Al3 (A = Pb and $\mathrm{Sn})$. Chemistry of Materials 26, 5394-5400 (Sept. 2014).

15. Jeon, N. J. et al. Solvent engineering for high-performance inorganicorganic hybrid perovskite solar cells. Nature Materials 13, 897-903 (Sept. 2014).

16. Jeon, N. J. et al. Compositional engineering of perovskite materials for high-performance solar cells. Nature 517, 476-+ (Jan. 2015).

17. Liu, M. Z., Johnston, M. B. \& Snaith, H. J. Efficient planar heterojunction perovskite solar cells by vapour deposition. Nature 501, 395-+ (Sept. 2013).

18. Zhou, H. P. et al. Interface engineering of highly efficient perovskite solar cells. Science 345, 542-546 (Aug. 2014).

19. Seebeck, T. J. Ueber die magnetische Polarisation der Metalle und Erze durch Temperaturdifferenz. Annalen der Physik 82, 253-286 (1826).

20. Pollock, D. D. The theory and properties of thermocouple elements / [by] D. D. Pollock. (Philadelphia, American Society for Testing and Materials, Philadelphia, 1971).

21. Peltier, J. Nouvelles Experiences sur la Caloriecete des Courans Electrique. Annali di Chimica LVI, 371-387 (1834).

22. Ibach, H. \& Lüth, H. Solid-State Physics: An Introduction to Principles of Materials Science (Springer Berlin Heidelberg: Berlin, Heidelberg, Berlin, Heidelberg, 2009).

23. Thomson, W. On a Mechanical Theory of Thermo-Electric Currents. Proceedings of the Royal society of Edinburgh 3, 91-98 (1857).

24. Nemir, D. \& Beck, J. On the Significance of the Thermoelectric Figure of Merit Z. Journal of Electronic Materials 39, 1897-1901 (Sept. 2010).

25. Vineis, C. J., Shakouri, A., Majumdar, A. \& Kanatzidis, M. G. Nanostructured Thermoelectrics: Big Efficiency Gains from Small Features. English. Advanced Materials 22, 3970-3980. ISSN: 0935-9648 (2010). 
26. Snyder, G. J. \& Toberer, E. S. Complex thermoelectric materials. Nature Materials 7, 105-114 (Feb. 2008).

27. Hosono, H. Nanomaterials: from research to applications (Elsevier, Oxford, 2006).

28. Mott, N. F. N. F. The theory of the properties of metals and alloys, / by N. F. Mott and H. Jones. (ed Jones, H.) (New York, Dover Publications, New York, 1958).

29. Pichanusakorn, P. \& Bandaru, P. Nanostructured thermoelectrics. English. Materials Science E Engineering R-Reports 67, 19-63. ISSN: 0927796X (2010).

30. Hicks, L. D. \& Dresselhaus, M. S. EFFECT OF QUANTUM-WELL STRUCTURES ON THE THERMOELECTRIC FIGURE OF MERIT. English. Physical Review B 47, 12727-12731. ISSN: 0163-1829 (1993).

31. Harman, T. C., Taylor, P. J., Walsh, M. P. \& LaForge, B. E. Quantum dot superlattice thermoelectric materials and devices. English. Science 297, 2229-2232. ISSN: 0036-8075 (2002).

32. Kanatzidis, M. G. Nanostructured Thermoelectrics: The New Paradigm? English. Chemistry of Materials 22, 648-659. ISSN: 0897-4756 (2010).

33. Mehta, R. J. et al. A new class of doped nanobulk high-figure-of-merit thermoelectrics by scalable bottom-up assembly. Nature Materials 11, 233-240 (Mar. 2012).

34. Tang, X. F. et al. Preparation and thermoelectric transport properties of high-performance p-type Bi2Te3 with layered nanostructure. Applied Physics Letters 90, 012102 (Jan. 2007).

35. Xie, W. J., Tang, X. F., Yan, Y. G., Zhang, Q. J. \& Tritt, T. M. Unique nanostructures and enhanced thermoelectric performance of melt-spun BiSbTe alloys. Applied Physics Letters 94, 102111 (Mar. 2009).

36. Zhao, X. B. et al. Bismuth telluride nanotubes and the effects on the thermoelectric properties of nanotube-containing nanocomposites. Applied Physics Letters 86, 062111 (Feb. 2005).

37. Cao, Y. Q., Zhao, X. B., Zhu, T. J., Zhang, X. B. \& Tu, J. P. Syntheses and thermoelectric properties of Bi2Te3/Sb2Te3 bulk nanocomposites with laminated nanostructure. Applied Physics Letters 92, 143106 (Apr. 2008). 
38. Stavila, V. et al. Wet-Chemical Synthesis and Consolidation of Stoichiometric Bismuth Telluride Nanoparticles for Improving the Thermoelectric Figure-of-Merit. Acs Applied Materials \& Interfaces 5, 6678-6686 (July 2013).

39. Soni, A. et al. Enhanced Thermoelectric Properties of Solution Grown Bi2Te3-xSex Nanoplatelet Composites. Nano Letters 12, 1203-1209 (Mar. 2012).

40. Scheele, M. et al. Synthesis and Thermoelectric Characterization of Bi2Te3 Nanoparticles. Advanced Functional Materials 19, 3476-3483 (Nov. 2009).

41. Zhao, L. D. et al. Ultrahigh power factor and thermoelectric performance in hole-doped single-crystal SnSe. Science 351, 141-144 (Jan. 2016).

42. Baranowski, L. L., Snyder, G. J. \& Toberer, E. S. Concentrated solar thermoelectric generators. Energy E Environmental Science 5, 9055-9067 (Oct. 2012).

43. Kraemer, D. et al. High-performance flat-panel solar thermoelectric generators with high thermal concentration. Nature Materials 10, 532538 (July 2011).

44. Chen, W. H., Wang, C. C., Hung, C. I., Yang, C. C. \& Juang, R. C. Modeling and simulation for the design of thermal-concentrated solar thermoelectric generator. Energy 64, 287-297 (Jan. 2014).

45. Green, M. A., Ho-Baillie, A. \& Snaith, H. J. The emergence of perovskite solar cells. Nature Photonics 8, 506-514 (July 2014).

46. Xing, G. C. et al. Long-Range Balanced Electron- and Hole-Transport Lengths in Organic-Inorganic CH3NH3PbI3. Science 342, 344-347 (Oct. 2013).

47. Stranks, S. D. et al. Electron-Hole Diffusion Lengths Exceeding 1 Micrometer in an Organometal Trihalide Perovskite Absorber. Science 342, 341-344 (Oct. 2013).

48. Dong, Q. F. et al. Electron-hole diffusion lengths $>175 \mathrm{mu} \mathrm{m}$ in solutiongrown CH3NH3PbI3 single crystals. Science 347, 967-970 (Feb. 2015).

49. Ponseca, C. S. et al. Organometal Halide Perovskite Solar Cell Materials Rationalized: Ultrafast Charge Generation, High and Microsecond-Long Balanced Mobilities, and Slow Recombination. Journal of the American Chemical Society 136, 5189-5192 (Apr. 2014). 
50. Stoumpos, C. C., Malliakas, C. D. \& Kanatzidis, M. G. Semiconducting Tin and Lead Iodide Perovskites with Organic Cations: Phase Transitions, High Mobilities, and Near-Infrared Photoluminescent Properties. Inorganic Chemistry 52, 9019-9038 (Aug. 2013).

51. De Wolf, S. et al. Organometallic Halide Perovskites: Sharp Optical Absorption Edge and Its Relation to Photovoltaic Performance. Journal of Physical Chemistry Letters 5, 1035-1039 (Mar. 2014).

52. Kim, Y. H. et al. Multicolored Organic/Inorganic Hybrid Perovskite Light-Emitting Diodes. Advanced Materials 27, 1248-1254 (Feb. 2015).

53. Tan, Z. K. et al. Bright light-emitting diodes based on organometal halide perovskite. Nature Nanotechnology 9, 687-692 (Sept. 2014).

54. Dong, R. et al. High-Gain and Low-Driving-Voltage Photodetectors Based on Organolead Triiodide Perovskites. Advanced Materials 27, 1912+ (Mar. 2015).

55. Dou, L. T. et al. Solution-processed hybrid perovskite photodetectors with high detectivity. Nature Communications 5, 5404 (Nov. 2014).

56. Fang, Y. J., Dong, Q. F., Shao, Y. C., Yuan, Y. B. \& Huang, J. S. Highly narrowband perovskite single-crystal photodetectors enabled by surfacecharge recombination. Nature Photonics 9, 679-+ (Oct. 2015).

57. Hu, X. et al. High-Performance Flexible Broadband Photodetector Based on Organolead Halide Perovskite. Advanced Functional Materials 24, 7373-7380 (Dec. 2014).

58. Maculan, G. et al. CH3NH3PbCl3 Single Crystals: Inverse Temperature Crystallization and Visible-Blind UV-Photodetector. Journal of Physical Chemistry Letters 6, 3781-3786 (Oct. 2015).

59. Zhu, H. M. et al. Lead halide perovskite nanowire lasers with low lasing thresholds and high quality factors. Nature Materials 14, 636-U115 (June 2015).

60. Deschler, F. et al. High Photoluminescence Efficiency and Optically Pumped Lasing in Solution-Processed Mixed Halide Perovskite Semiconductors. Journal of Physical Chemistry Letters 5, 1421-1426 (Apr. 2014).

61. Sutherland, B. R., Hoogland, S., Adachi, M. M., Wong, C. T. O. \& Sargent, E. H. Conformal Organohalide Perovskites Enable Lasing on Spherical Resonators. Acs Nano 8, 10947-10952 (Oct. 2014). 
62. Mettan, X. et al. Tuning of the Thermoelectric Figure of Merit of CH3NH3MI3 ( $\mathrm{M}=\mathrm{Pb}, \mathrm{Sn})$ Photovoltaic Perovskites. Journal of Physical Chemistry C 119, 11506-11510 (May 2015).

63. Pisoni, A. et al. Ultra-Low Thermal Conductivity in Organic-Inorganic Hybrid Perovskite CH3NH3PbI3. Journal of Physical Chemistry Letters 5, 2488-2492 (July 2014).

64. Physical Property Measurement System Thermal Transport Option User's Manual Quantum Design (2002).

65. Lu, W. G., Ding, Y., Chen, Y. X., Wang, Z. L. \& Fang, J. Y. Bismuth telluride hexagonal nanoplatelets and their two-step epitaxial growth. Journal of the American Chemical Society 127, 10112-10116 (July 2005).

66. Yuan, Q., Radar, K. \& Hussain, M. M. Acetic acid-confined synthesis of uniform three-dimensional (3D) bismuth telluride nanocrystals consisting of few-quintuple-layer nanoplatelets. Chemical Communications 47, 12131-12133 (2011).

67. Bos, J. W. G., Zandbergen, H. W., Lee, M. H., Ong, N. P. \& Cava, R. J. Structures and thermoelectric properties of the infinitely adaptive series (Bi-2)(m)(Bi2Te3)(n). Physical Review B 75, 195203 (May 2007).

68. Choi, S., Jin, H. \& Kim, S. SnS44- Metal Chalcogenide Ligand, S2Metal Free Ligand, and Organic Surface Ligand Toward Efficient CdSe Quantum Dot-Sensitized Solar Cells. Journal of Physical Chemistry C 118, 17019-17027 (July 2014).

69. Nag, A. et al. Metal-free Inorganic Ligands for Colloidal Nanocrystals: S2-, HS-, Se2-, HSe-, Te2-, HTe-, TeS32-, OH-, and NH2- as Surface Ligands. Journal of the American Chemical Society 133, 10612-10620 (July 2011).

70. Dong, A. G. et al. A Generalized Ligand-Exchange Strategy Enabling Sequential Surface Functionalization of Colloidal Nanocrystals. Journal of the American Chemical Society 133, 998-1006 (Feb. 2011).

71. Suehiro, S. et al. Solution-Processed Cu2ZnSnS4 Nanocrystal Solar Cells: Efficient Stripping of Surface Insulating Layers Using Alkylating Agents. Journal of Physical Chemistry C 118, 804-810 (Jan. 2014).

72. Sun, Y. M. et al. Impact of Surface-Bound Small Molecules on the Thermoelectric Property of Self-Assembled Ag2Te Nanocrystal Thin Films. Nano Letters 15, 3748-3756 (June 2015). 
73. Zhang, H. T. et al. Surfactant Ligand Removal and Rational Fabrication of Inorganically Connected Quantum Dots. Nano Letters 11, 5356-5361 (Dec. 2011).

74. Riha, S. C. et al. Photoelectrochemical Characterization of Nanocrystalline Thin-Film Cu2ZnSnS4 Photocathodes. Acs Applied Materials $\mathcal{E}$ Interfaces 3, 58-66 (Jan. 2011).

75. Delaizir, G. et al. A comparative study of Spark Plasma Sintering (SPS), Hot Isostatic Pressing (HIP) and microwaves sintering techniques on p-type Bi2Te3 thermoelectric properties. English. Materials Research Bulletin 47, 1954-1960. ISSN: 0025-5408 (2012).

76. Xiao, C. et al. Magnetic ions in wide band gap semiconductor nanocrystals for optimized thermoelectric properties. Materials Horizons 1, 81-86 (Jan. 2014).

77. Zhao, L. D. et al. Thermoelectrics with Earth Abundant Elements: High Performance p-type PbS Nanostructured with SrS and CaS. Journal of the American Chemical Society 134, 7902-7912 (May 2012).

78. CALLAWAY, J. Model for Lattice Thermal Conductivity at Low Temperatures. Physical Review 113, 1046-1051 (1959).

79. Liu, X. et al. Shape-Controlled Synthesis of $\operatorname{SnE}(E=S, S e)$ Semiconductor Nanocrystals for Optoelectronics. Chemistry of Materials 26, 35153521 (June 2014).

80. Burschka, J. et al. Sequential deposition as a route to high-performance perovskite-sensitized solar cells. Nature 499, 316-+ (July 2013).

81. Saidaminov, M. I. et al. High-quality bulk hybrid perovskite single crystals within minutes by inverse temperature crystallization. Nature Communications 6, 7586 (July 2015).

82. Shi, D. et al. Low trap-state density and long carrier diffusion in organolead trihalide perovskite single crystals. Science 347, 519-522 (Jan. 2015).

83. Shao, Y. H., Xiao, Z. G., Bi, C., Yuan, Y. B. \& Huang, J. S. Origin and elimination of photocurrent hysteresis by fullerene passivation in $\mathrm{CH} 3 \mathrm{NH} 3 \mathrm{PbI} 3$ planar heterojunction solar cells. Nature Communications 5, 5784 (Dec. 2014).

84. Dang, Y. Y. et al. Bulk crystal growth of hybrid perovskite material CH3NH3PbI3. Crystengcomm 17, 665-670 (2015). 
85. Saidaminov, M. I., Abdelhady, A. L., Maculan, G. \& Bakr, O. M. Retrograde solubility of formamidinium and methylammonium lead halide perovskites enabling rapid single crystal growth. Chemical Communications 51, 17658-17661 (2015).

86. Zhang, T. Y. et al. A facile solvothermal growth of single crystal mixed halide perovskite $\mathrm{CH} 3 \mathrm{NH} 3 \mathrm{~Pb}(\mathrm{Br} 1-x \mathrm{Clx})(3)$. Chemical Communications 51, 7820-7823 (2015).

87. Grancini, G. et al. CH3NH3PbI3 perovskite single crystals: surface photophysics and their interaction with the environment. Chemical Science 6, 7305-7310 (2015).

88. Mott, N. F. N. F. Electronic processes in ionic crystals / by N.F. Mott and R.W. Gurney. 2nd ed.. (ed Gurney, R. W. R. W.) (New York : Dover Publications, New York, 1964).

89. Lampert, M. A. Simplified Theory of Space-charge-limited Currents in an Insulator with Traps. Physical Review 103, 1648-1656 (1956).

90. (ed Ostroverkhova, O.) Handbook of organic materials for optical and (opto)electronic devices : properties and applications / edited by Oksana Ostroverkhova. 2013.

91. Jurchescu, O. D. \& Palstra, T. T. M. Crossover from one- to two-dimensional space-charge-limited conduction in pentacene single crystals. Applied Physics Letters 88, 122101 (Mar. 2006).

92. Edri, E., Kirmayer, S., Cahen, D. \& Hodes, G. High Open-Circuit Voltage Solar Cells Based on Organic-Inorganic Lead Bromide Perovskite. Journal of Physical Chemistry Letters 4, 897-902 (Mar. 2013).

93. Giorgi, G. \& Yamashita, K. Organic-inorganic halide perovskites: an ambipolar class of materials with enhanced photovoltaic performances. Journal of Materials Chemistry A 3, 8981-8991 (2015).

94. Nie, W. et al. Light-activated photocurrent degradation and self-healing in perovskite solar cells. Nature Communications 7, 11574 (May 2016).

95. Richardson, G. et al. Can slow-moving ions explain hysteresis in the current-voltage curves of perovskite solar cells? Energy $\mathcal{E}$ Environmental Science 9, 1476-1485 (2016).

96. Haruyama, J., Sodeyama, K., Han, L. Y. \& Tateyama, Y. First-Principles Study of Ion Diffusion in Perovskite Solar Cell Sensitizers. Journal of the American Chemical Society 137, 10048-10051 (Aug. 2015). 
97. Eames, C. et al. Ionic transport in hybrid lead iodide perovskite solar cells. Nature Communications 6, 7497 (June 2015).

98. Yuan, Y. B. \& Huang, J. S. Ion Migration in Organometal Trihalide Perovskite and Its Impact on Photovoltaic Efficiency and Stability. Accounts of Chemical Research 49, 286-293 (Feb. 2016).

99. Calado, P. et al. Evidence for ion migration in hybrid perovskite solar cells with minimal hysteresis. Nature Communications 7, 13831 (Dec. 2016).

100. Sze, S. M. \& Ng, K. K. Physics of Semiconductor Devices <https : / / books.google.co.nz/books? id=o4unkmHBHb8C> (Wiley, 2006).

101. Darby, B. L., Auguie, B., Meyer, M., Pantoja, A. E. \& Le Ru, E. C. Modified optical absorption of molecules on metallic nanoparticles at submonolayer coverage. Nature Photonics 10, 40-U54 (Jan. 2016).

102. Mancini, A. et al. CH3NH3SnxPb1-xBr3 Hybrid Perovskite Solid Solution: Synthesis, Structure, and Optical Properties. Inorganic Chemistry 54, 8893-8895 (Sept. 2015).

103. Briseno, A. L. et al. Patterning organic single-crystal transistor arrays. Nature 444, 913-917 (Dec. 2006).

104. Wang, G. et al. Wafer-scale growth of large arrays of perovskite microplate crystals for functional electronics and optoelectronics. Science advances 1, e1500613 (2015). 